\title{
EPITAXIAL CATI5O11 AND TIO2-B THIN FILMS FOR HIGH RATE LITHIUM-ION BATTERIES
}

\author{
by
}

\author{
Kui Zhang \\ A dissertation submitted in partial fulfillment \\ of the requirements for the degree of \\ Doctor of Philosophy \\ (Materials Science and Engineering) \\ in the University of Michigan \\ 2015
}

Doctoral Committee:

Professor Xiaoqing Pan, Chair

Associate Professor Bart M. Bartlett

Adjunct Professor George W. Graham

Assistant Professor Emmanouil Kioupakis 
My life is not my own. 
(C) Kui Zhang

2015 


\section{Dedication}

This dissertation is dedicated to all those who are on the trek to a brighter future. 


\section{Acknowledgements}

It was a difficult yet fruitful journey that led to my doctorate, and I could not have walked it without the love of my family. This is to you, my beautiful wife Yan Zhang and my dear son

\section{Rylan Siyuan Zhang.}

A million thanks to every member on my committee for the undoubted help they have offered every step of the way. My advisors Professor Xiaoqing Pan and Professor George W. Graham were directing the research efforts, steadily supporting my study, pointing out issues to be improved and areas to be polished, while leaving me plenty of freedom in the field, for which I am especially grateful.

Professor Bart M. Bartlett, along with his student Dr. Xiaoguang Hao, was very gracious to mentor me on the experimental setup and data interpretation in electrochemical testing. Their help was critical throughout the project in this thesis, as well as in the entire process of publishing the work.

Professor Emmanouil Kioupakis, along with his student Dylan Bayerl, was extremely helpful and a precious knowledge base to turn to, whenever I needed input from a theoretical point of view to guide my experimental efforts.

Professor Anton Van der Ven was the very reason we started to carefully investigate the $\mathrm{TiO}_{2}-\mathrm{B}$ material and the significance in its waterless synthesis, and has provided priceless guidance in theoretical modeling and understanding the physics.

My appreciation also goes to Professor Darrell G. Schlom and his research group at Cornell University for providing the high quality substrates with a variety of buffer layers; to Professor Ctirad Uher and his student Dr. Hang Chi for temperature dependent transport 
measurements; to Professor Kevin P. Pipe and his student Dr. Gun-Ho Kim for thermal conductivity measurements; and to Dr. Bing Liu at IMRA, Inc. for assistance in thin film thickness measurements and room temperature Hall measurements.

To Dr. Michael B. Katz and Dr. Baihai Li, thank you for being my fellow soldiers in the trench as this bronze story unfolded. It was a magical experience when I grew a film, Mike saw it as how the atoms stood, while Baihai calculated where they should be, and then I moved on to the next growth. The collaboration was so efficient that I enjoyed it far beyond I can describe.

To Sung Joo Kim, Jacob R. Jokisaari and Shuyi Zhang, thank you very much for making me the luckiest crystal grower on this planet. Your unbelievable capabilities in microscopy, and the amazingly short time in which you delivered all those stunning TEM images, have ensured that I know exactly what to tune for the next growth, and that we understand the microstructures at the atomic scale. This thesis, as well as most of my publications, is much more colorful with the images from you.

To Dr. Xianfeng Du, Dr. Kaixin Song, Yi Zhang (\#2) and Dr. Zhengbin Gu, thank you for working together with me in the process of target making and thin film deposition. The work would have been significantly slower without your help.

To Dr. Arnold L. Allenic and Dr. Wei Guo, countless thanks to you for being my mentor in crystal growth and thin film characterization techniques, and being my friends. I will always keep fond memories of ours in that underground PLD lab, and the vacuum pumps we tore apart.

To my labmates Dr. Yanbin Chen, Dr. Christopher T. Nelson, Dr. Obiefune K. Ezekoye, Dr. Yi Zhang (\#1), Linze Li and Mingjie Xu, thank you for all the help in numerous projects, experiments and discussions, and for the laughter and tears we shared over the years.

And to many other faculty members, graduate students, specialists, technicians and administrative staff here at the University of Michigan, especially at the Department of Materials Science and Engineering, thank you for making my graduate school experience unforgettable, so that I will forever be proud to call these hallways home. 


\section{Table of Contents}

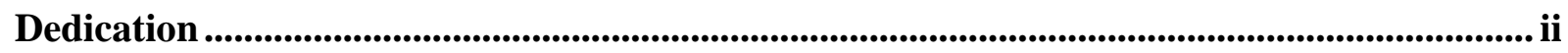

Acknowledgements .................................................................................................................................... iii

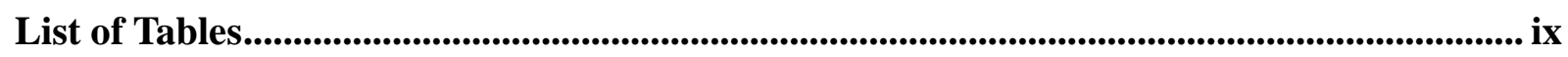

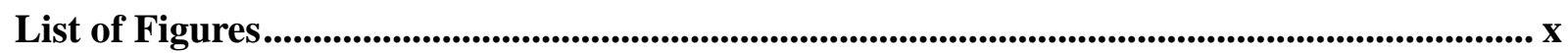

List of Appendices ............................................................................................................................................. xviii

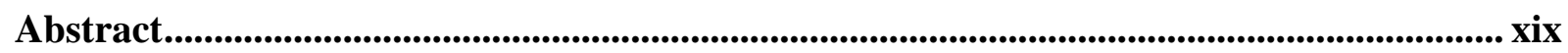

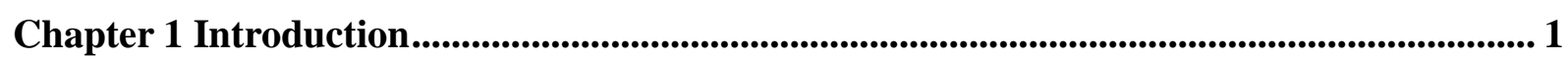

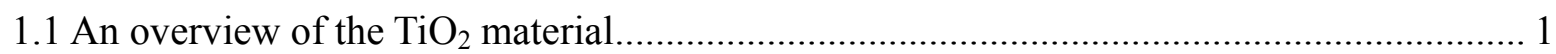

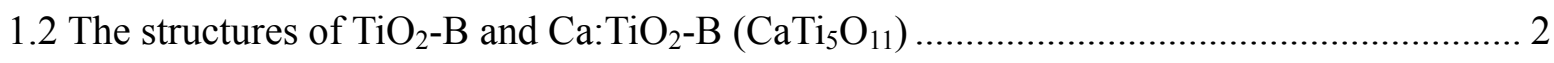

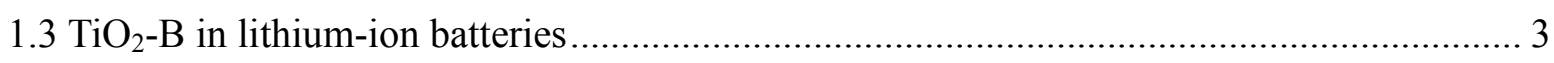

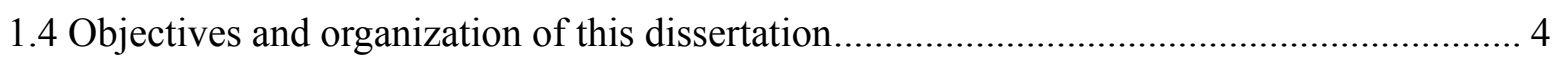

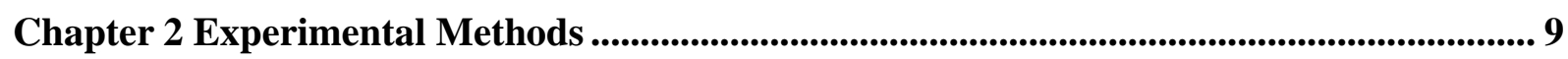

2.1 Epitaxial growth by pulsed laser deposition................................................................... 9

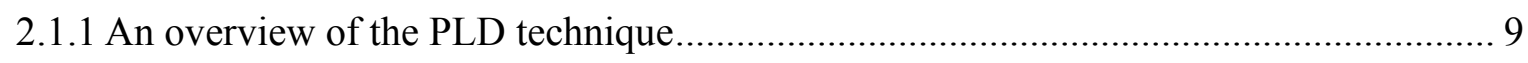

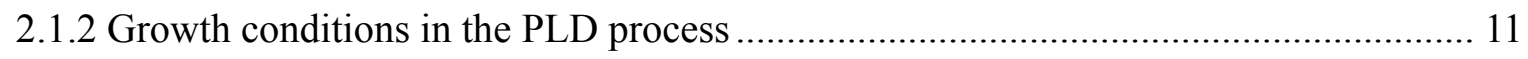

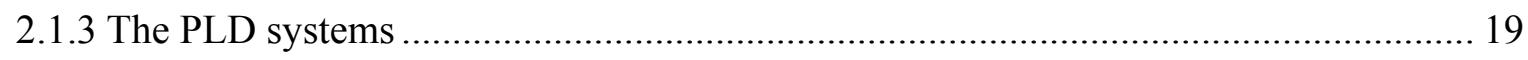

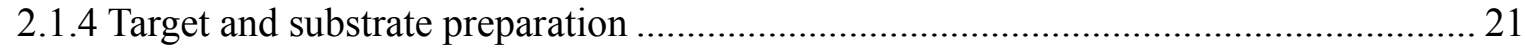




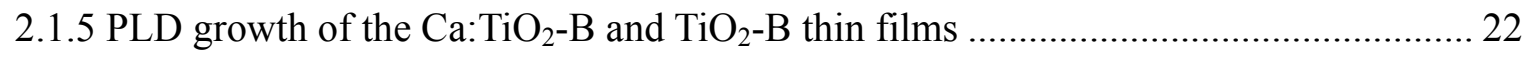

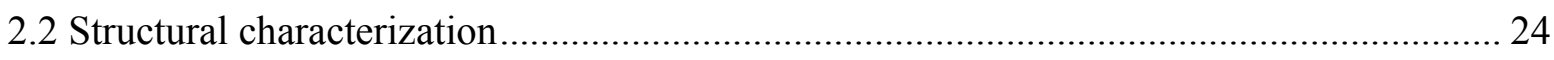

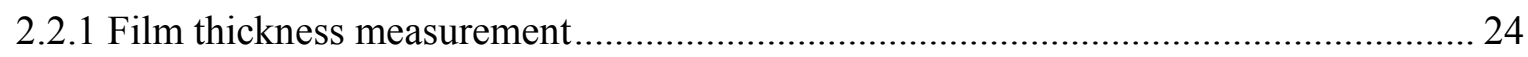

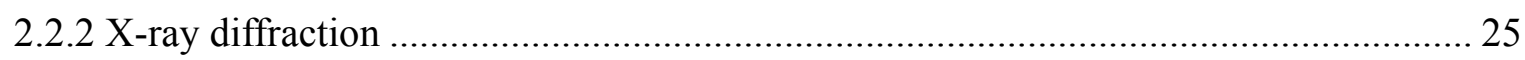

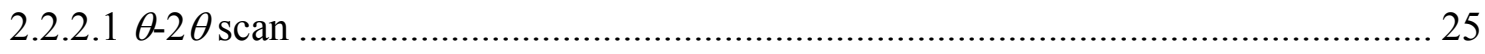

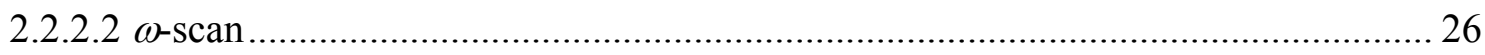

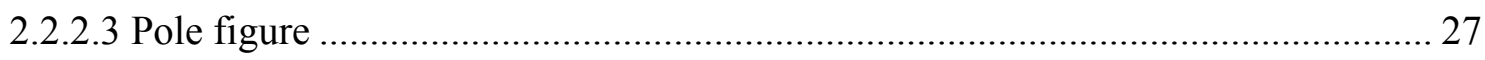

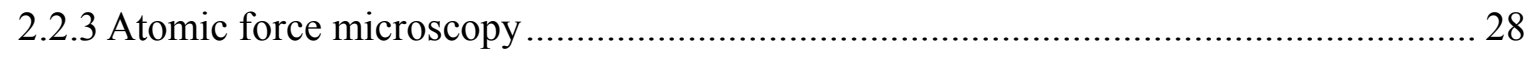

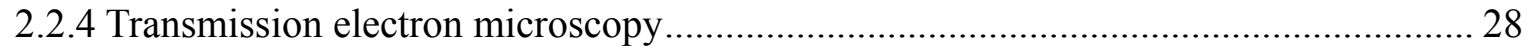

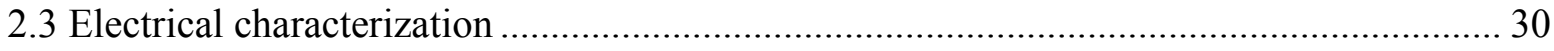

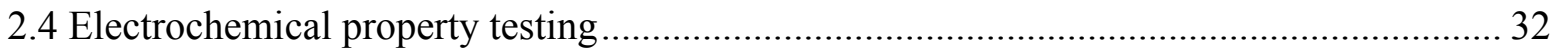

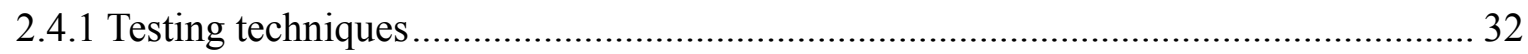

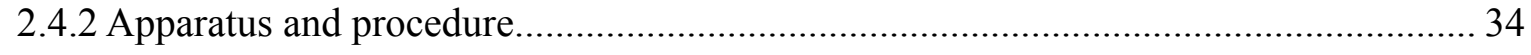

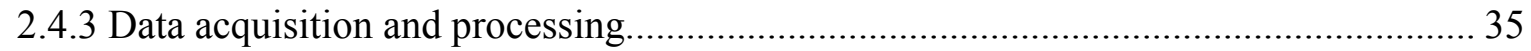

Chapter 3 Epitaxial Growth of High Quality Ca:TiO $2-\mathrm{B}\left(\mathrm{CaTi}_{5} \mathrm{O}_{11}\right)$ and $\mathrm{TiO}_{2}-\mathrm{B}$ Thin Films 49

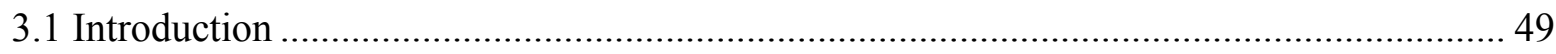

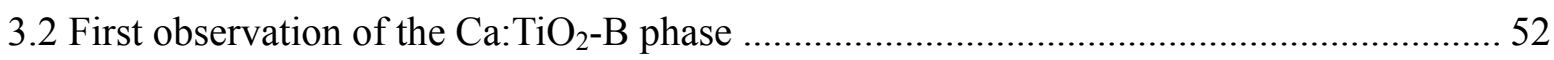

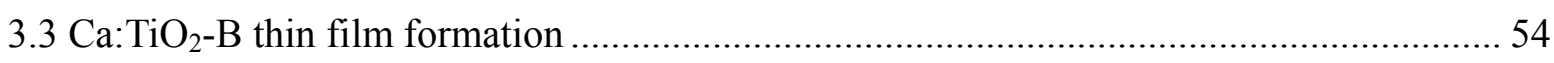

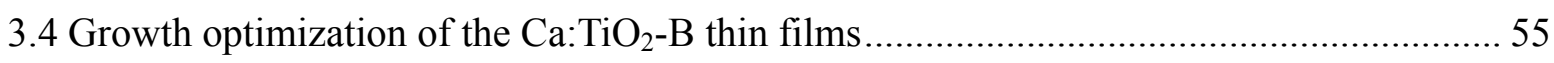

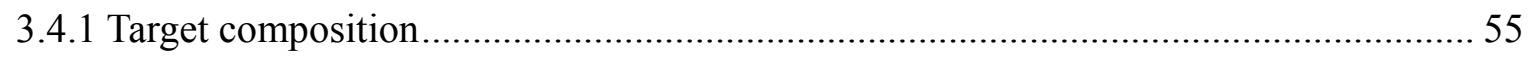

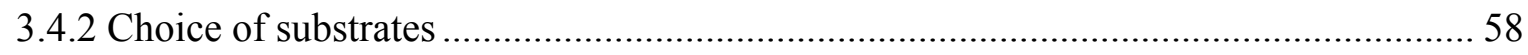

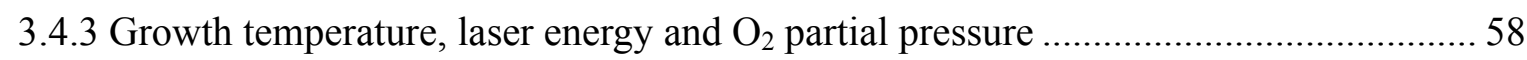

$3.5 \mathrm{TiO}_{2}-\mathrm{B}$ thin film growth on a $\mathrm{Ca}: \mathrm{TiO}_{2}-\mathrm{B}$ template layer............................................. 59

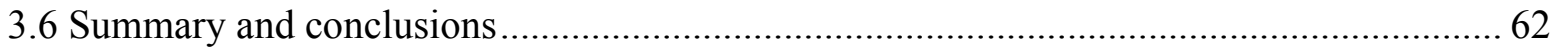

\section{Chapter 4 Epitaxial Ca:TiO $2-\mathrm{B}\left(\mathrm{CaTi}_{5} \mathrm{O}_{11}\right)$ and $\mathrm{TiO}_{2}-\mathrm{B}$ Thin Films as Anode Materials for}

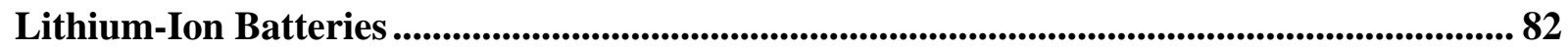

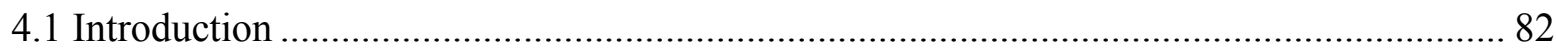

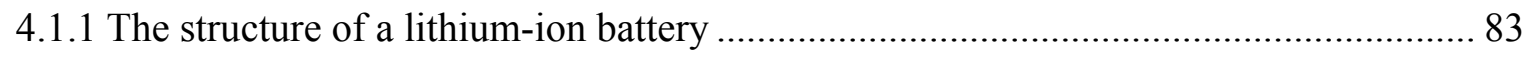

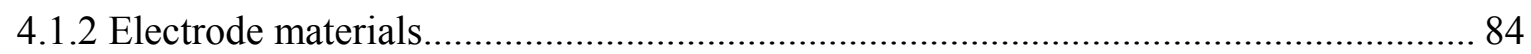




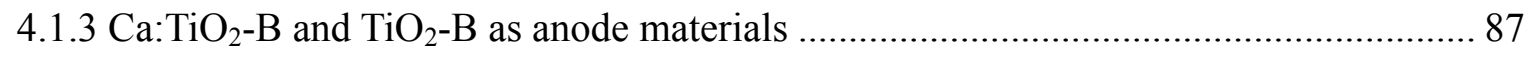

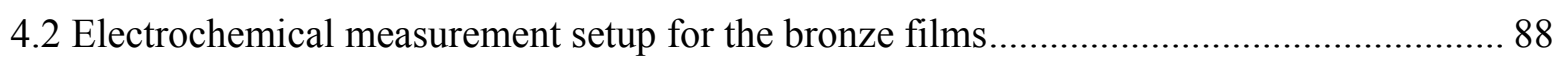

4.2.1 Bronze thin film preparation on $\mathrm{Nb}: \mathrm{SrTiO}_{3}$ substrates .............................................. 88

4.2.2 Top current collection configuration and data acquisition protocol ............................ 90

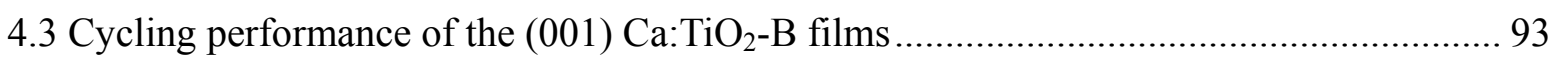

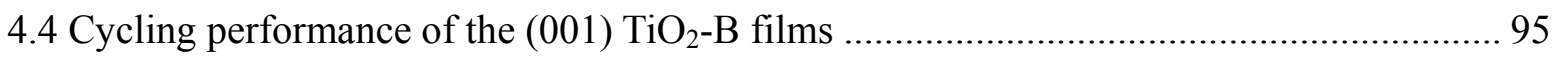

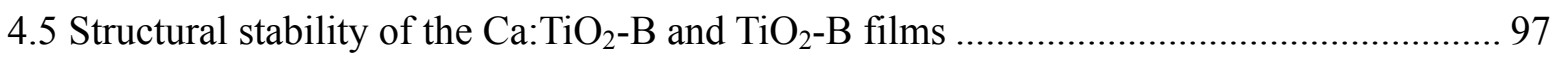

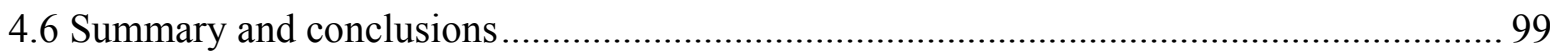

Chapter 5 Enhanced High Rate Performance in Ca:TiO ${ }_{2}-\mathrm{B}$ Thin Films Grown on (110)

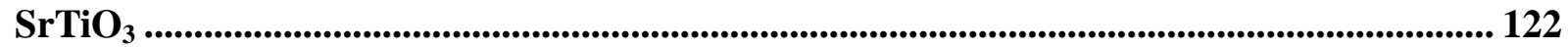

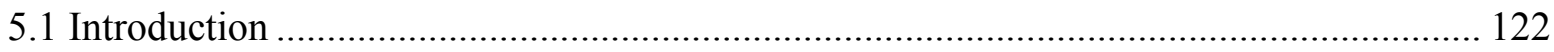

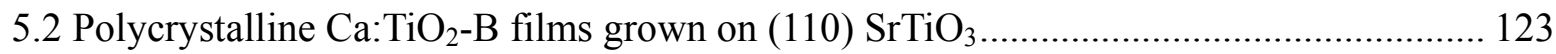

5.3 Enhanced battery performance in $\mathrm{Ca} \mathrm{TiO}_{2}-\mathrm{B}$ films grown on (110) $\mathrm{SrTiO}_{3} \ldots \ldots \ldots \ldots \ldots \ldots . . . . . . . .125$

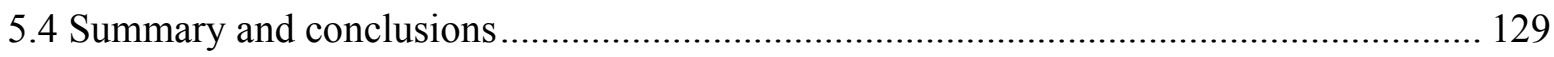

Chapter 6 High Rate Full-Cell Batteries Containing a Polycrystalline Ca: $\mathrm{TiO}_{2}-\mathrm{B}$ Anode and $\mathrm{LiFePO}_{4}$ or $\mathrm{LiMn}_{2} \mathrm{O}_{4}$ Cathodes ............................................................................................ 139

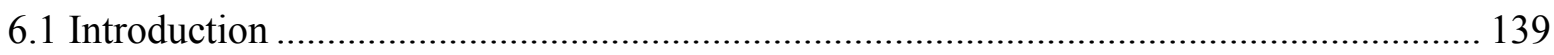

6.2 Polycrystalline $\mathrm{Ca}: \mathrm{TiO}_{2}$-B thin film anode vs. $\mathrm{LiFePO}_{4}$ slurry cathode ......................... 140

6.3 Polycrystalline $\mathrm{Ca}: \mathrm{TiO}_{2}-\mathrm{B}$ thin film anode vs. $\mathrm{LiMn}_{2} \mathrm{O}_{4}$ slurry cathode ......................... 142

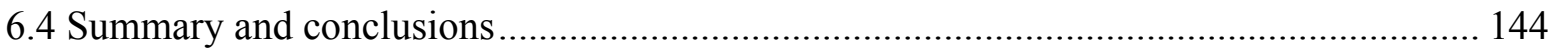

Chapter 7 Conclusions and Future Work...................................................................................... 154

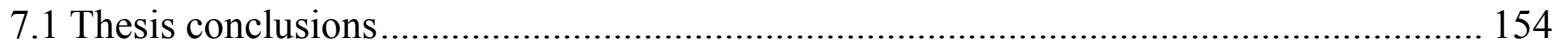

7.1.1 Epitaxial growth of (001) $\mathrm{Ca}_{\mathrm{TiO}_{2}} \mathrm{~B}$ and (001) $\mathrm{TiO}_{2}-\mathrm{B}$ thin films …........................ 154

7.1.2 Electrochemical properties of the $\mathrm{Ca}: \mathrm{TiO}_{2}-\mathrm{B}$ and $\mathrm{TiO}_{2}-\mathrm{B}$ thin films ........................ 155

7.1.3 Polycrystalline $\mathrm{Ca}: \mathrm{TiO}_{2}-\mathrm{B}$ thin film with enhanced high rate performance ................ 156

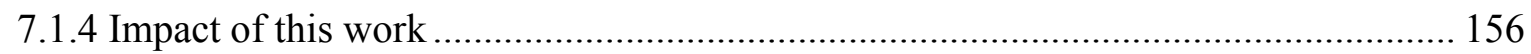

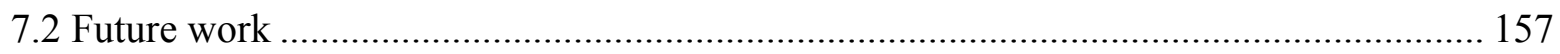

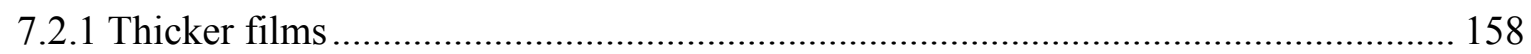

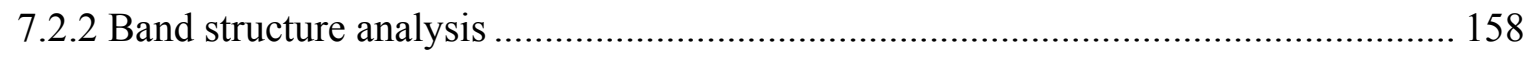


7.2.3 Increasing electrical conductivity 159

7.2.4 Origin of the pseudocapacitance 160

7.2.5 Divalent ion batteries 160

7.2.6 Related new phases 161

Appendix A TiO - -B Band Structure by First Principles Calculations... 163

Appendix B Enhanced Thermoelectric Properties in a Metal-Perovskite System 166

Bibliography 178 


\section{List of Tables}

Table 1.1 Structural parameters of $\mathrm{TiO}_{2}$ polymorphs, summarized from Ref. 27 and Ref. $40 \ldots \ldots .6$

Table 1.2 Atom positions in the $\mathrm{CaTi}_{5} \mathrm{O}_{11}$ structure................................................................ 7

Table 3.1 Lattice structures of the phases and substrates involved in this study. ....................... 63

Table 3.2 In-plane mismatch (calculated on a diagonal basis) between difference phases and the substrate surfaces. The preferred phase eventually adopted by the film deposited from a $(80 \%$ $\mathrm{TiO}_{2}+20 \% \mathrm{CaO}$ ) target on each specific substrate is set in bold.

Table 4.1 List of a few popular cathode materials. 101

Table 4.2 List of a few popular anode materials 102

Table 7.1 Electrical properties of a (001) $\mathrm{Ca}: \mathrm{TiO}_{2}-\mathrm{B}$ film and a (001) $\mathrm{TiO}_{2}-\mathrm{B} / \mathrm{Ca}: \mathrm{TiO}_{2}-\mathrm{B}$ dual layer film both grown on (001) $\mathrm{SrTiO}_{3}$ substrates at $800{ }^{\circ} \mathrm{C}$. 162 


\section{List of Figures}

Figure 1.1 Crystal structures of regular $\mathrm{TiO}_{2}-\mathrm{B}$ (top row) and $\mathrm{Ca}: \mathrm{TiO}_{2}-\mathrm{B}\left(\mathrm{CaTi}_{5} \mathrm{O}_{11}\right.$, bottom row) projected along [100], [010] and [001] directions, respectively. These drawings were produced using VESTA. 8

Figure 2.1 Schematic of a typical PLD system (reproduced and modified from a schematic drawing available on http://www.egr.msu.edu/).

Figure 2.2 Photographs of (a) the excimer laser (COMPex 205), (b) the Neocera PLD system, (c) the laser plume captured in the Neocera system, and (d) the Thermionics system. This figure is reproduced from Ref. 59.

Figure 2.3 Schematic showing the relationship between the diffractometer axes and the substrate reference frame in the XRD measurements (modified from Ref. 60).

Figure 2.4 Sample geometries for Van der Pauw resistivity and Hall effect measurements. The cloverleaf design will have the lowest error due to its smaller effective contact size, but it is more difficult to fabricate than a square or rectangle [from NIST, Ref. 56]. The configuration used in this study is (b)

Figure 2.5 (a) The current applied on the battery cell in a galvanostatic cycling (GC) experiment [from the VersaStudio software]. (b) Typical current and potential profiles of a battery in a GC experiment.

Figure 2.6 (a) The voltage applied on the battery cell in a cyclic voltammetry (CV) experiment [from the VersaStudio software]. (b) A typical CV curve of a battery in a CV experiment. 44

Figure 2.7 (a) Internal structure of the commercial battery test cell (EL-CELL ECC-STD, image credit: http://el-cell.com/). (b) A photograph of the VersaSTAT MC 4-channel electrochemical testing system. [Princeton Applied Research: user manual].....

Figure 2.8 (a) The two-terminal connection. (b) The three-terminal connection. [Princeton Applied Research: user manual] 
Figure 2.9 Screenshot of the VersaStudio software in a galvanostatic cycling experiment on a (001) $\mathrm{Ca}: \mathrm{TiO}_{2}-\mathrm{B}$ thin film. The current is set at $14.1 \mu \mathrm{A}$, and the voltage window is $1-3 \mathrm{~V}$.

Figure 2.10 Screenshot of the VersaStudio software in a cyclic voltammetry experiment on a (001) $\mathrm{TiO}_{2}-\mathrm{B}$ thin film. The voltage window is $1-3 \mathrm{~V}$.

Figure 3.1 (a) $\mathrm{TiO}_{2}$-anatase and $\mathrm{TiO}_{2}$-B inclusions in the $\mathrm{CaTi}_{0.95} \mathrm{Pt}_{0.05} \mathrm{O}_{3}$ thin film after reduction treatment. (b-c) Calculated pseudo-ternary phase diagrams for the $\mathrm{CaO}_{\mathrm{x}}-\mathrm{TiO}_{\mathrm{y}}-\mathrm{PtO}_{z}$ system. [Figures reproduced from Ref. 62]

Figure 3.2 (a) $\mathrm{Ca}: \mathrm{TiO}_{2}$ - $\mathrm{B}$ phase inclusion in the $\mathrm{CaTi}_{0.95} \mathrm{Pt}_{0.05} \mathrm{O}_{3}$ thin film after reduction treatment. The arrow marks a Ti-Ca layer in the $\mathrm{Ca}: \mathrm{TiO}_{2}-\mathrm{B}$ structure. The dashed line marks the interface between $\mathrm{Ca}: \mathrm{TiO}_{2}-\mathrm{B}$ and simple $\mathrm{TiO}_{2}-\mathrm{B}$. (b) A large $\mathrm{Ca}: \mathrm{TiO}_{2}-\mathrm{B}$ grain observed in a $\mathrm{CaTi}_{0.95} \mathrm{Rh}_{0.05} \mathrm{O}_{3}$ thin film after reduction treatment. [TEM image credit: Michael B. Katz]...... 66

Figure 3.3 (a) HAADF STEM image of a (001) anatase film grown on a (001) $\mathrm{SrTiO}_{3}$ substrate by PLD. (b) The crystal structure of anatase projected along its [100] direction. [TEM image credit: Michael B. Katz].

Figure 3.4 STEM images of a thin film deposited on (001) $\mathrm{SrTiO}_{3}$ using a $\left(\mathrm{TiO}_{2} 90 \%+\mathrm{CaO}\right.$ $10 \%$ ) target, showing a few grains in the $\mathrm{Ca}: \mathrm{TiO}_{2}-\mathrm{B}$ structure. (a-b) Bright field images. (c-d) Dark field images. [TEM image credit: Michael B. Katz] 68

Figure 3.5 HAADF STEM images of a film deposited from a $\left(\mathrm{TiO}_{2} 90 \%+\mathrm{CaO} 10 \%\right)$ target showing the co-existence of $\mathrm{Ca}: \mathrm{TiO}_{2}-\mathrm{B}$ and anatase. (a) A low magnification image of the film. (b) A higher magnification image showing a $\mathrm{Ca}: \mathrm{TiO}_{2}-\mathrm{B}$ grain in a different orientation from its neighboring grain, which is encompassed by the dashed line. (c) More instances showing the grain rotation about the $c$-axis of $\mathrm{Ca}: \mathrm{TiO}_{2}$-B. [TEM image credit: Michael B. Katz]......

Figure 3.6 XRD patterns of thin films deposited from targets of different compositions. The 20\% $\mathrm{CaO}\left(80 \% \mathrm{TiO}_{2}\right.$, by mole) target resulted in a $(001) \mathrm{Ca}: \mathrm{TiO}_{2}-\mathrm{B}$ film with highest crystalline quality and only a trace amount of anatase. Vertical dashed lines mark the peak positions of the anatase phase.

Figure 3.7 Crystal structure projected along [010] (left) and [100] (right) directions, showing the interfacial atomic structure and epitaxial relationship between $\mathrm{Ca}: \mathrm{TiO}_{2}-\mathrm{B}(001)$ and $\mathrm{SrTiO}_{3}(001)$ planes.

Figure 3.8 HAADF STEM images of a (001) Ca: $\mathrm{TiO}_{2}-\mathrm{B}$ film deposited from a $\left(\mathrm{TiO}_{2} 80 \%+\mathrm{CaO}\right.$ $20 \%$ ) target on a (001) $\mathrm{SrTiO}_{3}$ substrate. (a) A low magnification image of the film with a smooth surface. (b) High magnification images showing the microstructure of the film. The arrows mark the stacking faults. (c) An anti-phase boundary caused by the shift of the Ti-Ca layer in two adjacent grains, marked by the arrows. The yellow dashed line depicts a discontinuous Ti-Ca 
Figure 3.9 XRD patterns of the films deposited from a $20 \% \mathrm{CaO}$ target on 5 different substrates. Growth conditions were the same as those used to grow the films in Figure 3.6. Substrate peaks are labeled in green, and anatase/rutile peaks in blue. Vertical dashed lines are drawn to mark peak positions for comparison.

Figure 3.10 XRD patterns of the films deposited from a $10 \% \mathrm{CaO}$ target on (001) $\mathrm{SrTiO}_{3}$ substrates under different growth temperatures from 600 to $900{ }^{\circ} \mathrm{C}$. Substrates peaks are labeled in green, anatase peaks in blue. Vertical dashed lines are drawn to mark peak positions for comparison.

Figure $3.11 \mathrm{XRD}$ patterns of the films deposited from a $10 \% \mathrm{CaO}$ target on (001) $\mathrm{SrTiO}_{3}$ substrates under different conditions: a) laser fluence from 3.4 to $6.8 \mathrm{~J} \mathrm{~cm}^{-2}$; b) $\mathrm{O}_{2}$ partial pressure from vacuum to 100 mTorr. Substrates peaks are labeled in green, anatase peaks in blue. Vertical dashed lines are drawn to mark peak positions for comparison.

Figure 3.12 The atomic model projected along $\mathrm{Ca}$ : $\mathrm{TiO}_{2}-\mathrm{B}$ 's $b$-axis showing the epitaxial relationship between $\mathrm{TiO}_{2}-\mathrm{B}$ and $\mathrm{Ca}: \mathrm{TiO}_{2}-\mathrm{B}$. The dashed line indicates the interface between the two phases, where they align their respective (001) planes with each other. 76

Figure 3.13 (001) $\mathrm{TiO}_{2}-\mathrm{B}$ grown on a (001) $\mathrm{Ca}: \mathrm{TiO}_{2}-\mathrm{B}$ template. a) XRD patterns of the dual layer films grown on various substrates. The $\mathrm{Ca}: \mathrm{TiO}_{2}-\mathrm{B}$ template was deposited from a $20 \% \mathrm{CaO}$ target. b-c) HAADF STEM images of a dual layer film grown on a $\mathrm{SrTiO}_{3}(001)$ substrate. The dashed line in b) indicates the interface. [TEM image credit: Michael B. Katz] .....

Figure 3.14 (a) High-resolution STEM images of defects at the interface between $\mathrm{TiO}_{2}-\mathrm{B}$ and $\mathrm{Ca}: \mathrm{TiO}_{2}-\mathrm{B}$ that generate the two adjacent $\mathrm{TiO}_{2}-\mathrm{B}$ grains orienting in opposite directions. (b) Two missing Ti-Ca layers result in the adjacent $\mathrm{TiO}_{2}-\mathrm{B}$ grains stacking in the same direction. Scale bars are $5 \mathrm{~nm}$. [TEM image credit: Sung Joo Kim].

Figure 3.15 (a) FWHM of the $\mathrm{TiO}_{2}$-B 002 peak as a function of the layer thickness (solid dots), and the average grain size calculated from the FWHM using Scherrer equation (open squares). The dashed line indicates the situation when grain size equals film thickness. (b) $\mathrm{TiO}_{2}-\mathrm{B} 002$ FWHM as a function of the $\mathrm{Ca}: \mathrm{TiO}_{2}-\mathrm{B}$ template thickness.

Figure $3.16 \mathrm{XRD} \theta-2 \theta$ pattern of a $(001) \mathrm{TiO}_{2}-\mathrm{B} / \mathrm{Ca}: \mathrm{TiO}_{2}-\mathrm{B}$ dual layer film grown on a $\mathrm{SrTiO}_{3}$ buffered (100) Si substrate. 80

Figure 3.17 AFM images (tapping mode) showing the surfaces of (a) a $\mathrm{Ca}: \mathrm{TiO}_{2}-\mathrm{B}$ film and (b) a $\mathrm{TiO}_{2}-\mathrm{B}$ film (on top of a $\mathrm{Ca}: \mathrm{TiO}_{2}-\mathrm{B}$ template), respectively, both grown on (001) $\mathrm{SrTiO}_{3}$ substrates. Scan area is $5 \mu \mathrm{m} \times 5 \mu \mathrm{m}$ for both. 
Figure 4.2 Dendritic growth of lithium during its electro-deposition. [From Ref. 132].

Figure 4.3 As-grown morphology of both the (001) $\mathrm{Ca}_{\mathrm{TiO}} \mathrm{Tin}$ thin film and the (001) $\mathrm{TiO}_{2}-\mathrm{B} / \mathrm{Ca}: \mathrm{TiO}_{2}-\mathrm{B}$ dual layer film on (100) $\mathrm{SrTiO}_{3}$ substrate. (a) Low magnification STEM image of a $\mathrm{Ca}: \mathrm{TiO}_{2}-\mathrm{B}$ film with $\sim 75 \mathrm{~nm}$ thickness. (b) Low magnification STEM image of a $\mathrm{TiO}_{2}$-B film $(\sim 65 \mathrm{~nm})$ grown on top of a thin $\mathrm{Ca}: \mathrm{TiO}_{2}-\mathrm{B}$ template layer $(\sim 15 \mathrm{~nm})$. The dashed line was drawn to locate the interface. [TEM image credit: Michael B. Katz] 105

Figure 4.4 Structural characterization of $\mathrm{Ca}: \mathrm{TiO}_{2}-\mathrm{B}$ and regular $\mathrm{TiO}_{2}-\mathrm{B}$. (a) XRD pattern of a (001) $\mathrm{Ca}_{\mathrm{TiO}_{2}} \mathrm{~B}$ thin film grown on a (001) $\mathrm{SrTiO}_{3}$ substrate at $800{ }^{\circ} \mathrm{C}$ by PLD. The hetero-epitaxial relationship is [100](001) Ca:TiO2-B $_{\|}[100](001)_{\mathrm{SrTiO}_{3}}$. (b) HAADF STEM image of the $\mathrm{Ca}: \mathrm{TiO}_{2}-\mathrm{B}$ phase. (c) XRD pattern of a regular (001) $\mathrm{TiO}_{2}-\mathrm{B}$ thin film grown on top of a thin $\mathrm{Ca}: \mathrm{TiO}_{2}-\mathrm{B}$ template layer on a (001) $\mathrm{SrTiO}_{3}$ substrate under the same PLD conditions. (d) HAADF STEM image of the dual layer structure. [TEM image credit: Michael B. Katz]........ 106

Figure 4.5 Bright field STEM image of a (001) $\mathrm{TiO}_{2}-\mathrm{B} / \mathrm{Ca}$ : $\mathrm{TiO}_{2}-\mathrm{B}$ dual layer film grown on a (001) $\mathrm{Nb}: \mathrm{SrTiO}_{3}$ substrate. Many small anatase grains formed at the interface. [TEM image credit: Michael B. Katz]. 107

Figure 4.6 (a) Low magnification and (b) high magnification HAADF STEM images showing a (001) $\mathrm{TiO}_{2}-\mathrm{B} / \mathrm{Ca}: \mathrm{TiO}_{2}-\mathrm{B}$ dual layer film grown on a (001) $\mathrm{Nb}: \mathrm{SrTiO}_{3}$ substrate with a $\mathrm{SrTiO}_{3}$ buffer layer. [TEM image credit: Michael B. Katz]... 108

Figure 4.7 Top current collector configuration for electrochemical measurements. Most battery performance results reported in this thesis were acquired using such configuration from films grown on insulating $10 \times 10 \mathrm{~mm}^{2} \mathrm{SrTiO}_{3}$ substrates. 109

Figure 4.8 Cycling performance of a bare $\mathrm{Nb}: \mathrm{SrTiO}_{3}(001)$ substrate at a $1 \mathrm{C}$ rate for 100 cycles in a voltage window of 1-3 $\mathrm{V}$ versus Li metal.

Figure 4.9 Comparison of discharge capacities of the $\mathrm{TiO}_{2}-\mathrm{B}$ thin film using two different test configurations. Capacity contribution from the $\mathrm{Ca}: \mathrm{TiO}_{2}-\mathrm{B}$ template layer was subtracted in both configurations; capacity of the conductive $\mathrm{Nb}: \mathrm{SrTiO}_{3}$ substrate was subtracted for the bottom collector configuration.

Figure $4.10 \mathrm{Cu}$ grid surface at the charged state. (a) Scanning electron microscopy image showing the surface of the $\mathrm{Cu}$ wire on the $\mathrm{Ca}: \mathrm{TiO}_{2}-\mathrm{B}$ thin film. This film was fully charged with $\mathrm{Li}^{+}$at the rate of $1000 \mathrm{C}$, and removed from the cell at a half cycle to be examined. No obvious $\mathrm{Li}$ plating was observed. (b) X-ray energy dispersive spectrum taken on the $\mathrm{Cu}$ wire. Carbon and fluorine are from the residue of $\mathrm{LiPF}_{6}$ electrolyte. 112

Figure 4.11 Voltage profile with $\mathrm{Li}$ content at the 8th cycle of a (001) $\mathrm{Ca}: \mathrm{TiO}_{2}-\mathrm{B}$ thin film grown on a (001) $\mathrm{SrTiO}_{3}$ substrate versus Li metal at a rate of $33.5 \mathrm{~mA} \mathrm{~g}^{-1}$ between $1.0-3.0 \mathrm{~V}$. 113 
Figure 4.12 Schemes showing idealized voltage and differential capacity $(\mathrm{d} C / \mathrm{d} V)$ profiles for three basic charge storage mechanisms. [From Ref. 38] ...

Figure 4.13 Cycling performance and Coulombic efficiency of a (001) $\mathrm{Ca}_{2} \mathrm{TiO}_{2}-\mathrm{B}$ thin film grown on a (001) $\mathrm{SrTiO}_{3}$ substrate at $10 \mathrm{C}\left(1 \mathrm{C}=294 \mathrm{~mA} \mathrm{~g}^{-1}\right)$ for 100 cycles. The voltage window is $1-3 \mathrm{~V}$. 115

Figure 4.14 (a) Voltage profile with $\mathrm{Li}$ content at the 8th cycle of a (001) $\mathrm{TiO}_{2}-\mathrm{B}$ thin film grown with a $\mathrm{Ca}: \mathrm{TiO}_{2}-\mathrm{B}$ template layer on a (001) $\mathrm{SrTiO}_{3}$ substrate versus $\mathrm{Li}$ metal at a $\mathrm{C} / 10$ rate $\left(1 \mathrm{C}=335 \mathrm{~mA} \mathrm{~g}^{-1}\right)$ between 1.0-3.0 V. (b) Slow rate $(\mathrm{C} / 12)$ cycling results for 18 cycles........... 116

Figure 4.15 (a) Cyclic voltammograms (CVs) of a (001) $\mathrm{TiO}_{2}-\mathrm{B}$ thin film using the top $\mathrm{Cu}$ grid current collector at different scan rates. Three cycles are shown for each rate, demonstrating good repeatability of the measurement. Signature $\mathrm{TiO}_{2}-\mathrm{B}$ peaks were observed. (b) Specific capacitance calculated by integrating the CVs at different scan rates for both the oxidation and reduction curves. 117

Figure 4.16 XRD $\theta-2 \theta$ patterns before and after cycling of (a) a (001) $\mathrm{Ca}: \mathrm{TiO}_{2}-\mathrm{B}$ film and (b) a (001) $\mathrm{TiO}_{2}-\mathrm{B} / \mathrm{Ca}: \mathrm{TiO}_{2}-\mathrm{B}$ dual layer film on (001) $\mathrm{SrTiO}_{3}$. 118

Figure 4.17 Before and after cycling structural comparison of a (001) $\mathrm{TiO}_{2}-\mathrm{B} / \mathrm{Ca}: \mathrm{TiO}_{2}-\mathrm{B}$ dual layer film grown on (001) $\mathrm{SrTiO}_{3}$. A fracture at the interface is clearly seen. Such fractures were observed throughout the post-cycling film. [TEM image credit: a) Michael B. Katz; b) Sung Joo Kim] 119

Figure 4.18 Fractures throughout the film caused by charging and discharging: lower magnification STEM image of the same sample as in Figure 4.17(b) showing more fractures in the (001) $\mathrm{TiO}_{2}-\mathrm{B} / \mathrm{Ca}: \mathrm{TiO}_{2}-\mathrm{B}$ dual layer film after cycling. The red arrows point to some of the fractures. [TEM image credit: Sung Joo Kim]. 120

Figure 4.19 Lattice changes due to $\mathrm{Li}^{+}$intercalation. XRD fine scans of (001) bronze films grown on (100) $\mathrm{SrTiO}_{3}$ substrates. Solid curves are fittings of the experimental data. (a) The $\mathrm{Ca}: \mathrm{TiO}_{2}-\mathrm{B} 006$ peak. A peak position shift indicating an increase of $c$ and a peak broadening (FWHM: full width at half maximum) were observed. b) The $\mathrm{TiO}_{2}-\mathrm{B} 001$ peak of a $\mathrm{TiO}_{2}-\mathrm{B} / \mathrm{Ca}: \mathrm{TiO}_{2}-\mathrm{B}$ dual layer film. A peak position shift indicating a slight decrease of $c$, and a peak broadening were observed.... 121

Figure 5.1 (a) Bright field STEM image of a $\mathrm{Ca}: \mathrm{TiO}_{2}-\mathrm{B}$ film grown on a (110) $\mathrm{SrTiO}_{3}$ substrate. (b-c) HAADF STEM images showing different grains in the film. The angles between the $a-b$ planes of different grains and the substrate surface are labeled. The yellow triangle in (c) marks a small $\mathrm{CaTiO}_{3}$ grain at the interface. [TEM image credit: Michael B. Katz] ............................. 130

Figure 5.2 (a) Pole figure of the polycrystalline $\mathrm{Ca}: \mathrm{TiO}_{2}-\mathrm{B}$ film grown on a (110) $\mathrm{SrTiO}_{3}$ 
substrate. The $2 \theta$ angle was set at $30.22^{\circ}$ for $\mathrm{Ca}: \mathrm{TiO}_{2}-\mathrm{B} 006$ peak. The $\phi$ angle was scanned over $0-355^{\circ}$ at a $5^{\circ}$ step; the $\chi$ angle was scanned over $0-75^{\circ}$ at a $2.5^{\circ}$ step. (b) Schematic drawing of the pole figure experiment setup. 131

Figure 5.3 (a) Schematic drawing of the bronze film growth on a (110) $\mathrm{SrTiO}_{3}$ substrate. (b) HAADF STEM image showing the cubic $\mathrm{CaTiO}_{3}$ phase formation at the interface. See Ref. 71 for more details.

Figure 5.4 Structure an inclined $\mathrm{Ca}: \mathrm{TiO}_{2}-\mathrm{B}$ film grown on a (110) $\mathrm{SrTiO}_{3}$ substrate. a) HAADF STEM image of a region near a grain boundary. b) Lower magnification STEM image showing the polycrystalline nature of the film. Different grains have channels along different directions exposed at the surface. c) HAADF STEM image showing a region near the surface after aggressive cycling for more than 60 days. No significant structural degradation, either on the surface or in the film, was observed. [TEM image credit: Michael B. Katz].

Figure 5.5 Electrochemical performance of an inclined $\mathrm{Ca}: \mathrm{TiO}_{2}-\mathrm{B}$ film grown on a (110) $\mathrm{SrTiO}_{3}$ substrate. (a) Charge-discharge voltage profiles of the 5 th cycle at each current rate from $1 \mathrm{C}$ to $600 \mathrm{C}\left(1 \mathrm{C}=335 \mathrm{~mA} \mathrm{~g}^{-1}\right)$. Dashed lines depict the profiles at $1 \mathrm{C}$ immediately following the last cycle at the highest rate. (b) Capacity retention at constant $60 \mathrm{C}$ and $80 \mathrm{C}$ rates for 200 cycles in a voltage window of 1-3 V. 134

Figure 5.6 Long term cyclability of a $62 \mathrm{~nm}$ polycrystalline $\mathrm{Ca}: \mathrm{TiO}_{2}-\mathrm{B}$ thin film vs. Li metal at a $60 \mathrm{C}$ rate. The voltage window is $1-3 \mathrm{~V}$. 135

Figure 5.7 Rate capability comparison: discharge capacity at the 20th cycle of both $\mathrm{TiO}_{2}-\mathrm{B}$ and $\mathrm{Ca}: \mathrm{TiO}_{2}-\mathrm{B}$ with different orientation at increasing rates. Respective film thicknesses are labeled. Solid lines are guides for the eyes. Inset: potential profiles at the 5th cycle of (001) $\mathrm{TiO}_{2}$-B and $\mathrm{Ca}: \mathrm{TiO}_{2}-\mathrm{B}$ films at $\mathrm{C} / 10$ rate $\left(33.5 \mathrm{~mA} \mathrm{~g}^{-1}\right)$...... 136

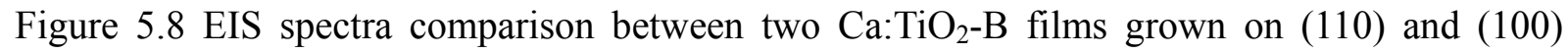
$\mathrm{SrTiO}_{3}$, respectively. The experimental data (open symbols) has been fitted to the equivalent circuit in the inset, which includes solution resistance $\left(R_{\mathrm{S}}\right)$, an $R C$ circuit representing charge transfer, and a Warburg impedance representing ion diffusion. 137

Figure 5.9 Cyclic voltammetry measurements for a polycrystalline $\mathrm{Ca}: \mathrm{TiO}_{2}-\mathrm{B}$ thin film grown on (110) $\mathrm{SrTiO}_{3}$ at different scan rates. Three cycles are shown for each rate, demonstrating good repeatability of the measurement. 138

Figure 6.1 Cycling performance of the homemade $\mathrm{LiFePO}_{4}$ slurry electrode vs. Li counter electrode at a rate of $47.4 \mathrm{~mA} \mathrm{~g}^{-1}$. The voltage window is $2.7-4.2 \mathrm{~V}$....... 145

Figure 6.2 (a) Charge-discharge voltage profiles at the 5 th cycle of the $\mathrm{LiFePO}_{4}$ slurry electrode vs. $\mathrm{Li}$ counter electrode at a rate of $47.4 \mathrm{~mA} \mathrm{~g}^{-1}$. The voltage window is 2.7-4.2 V. (b) Cyclic 
voltammograms (CVs) at different scan rates. Five cycles are shown for each rate.

Figure 6.3 Charge and discharge capacities vs. cycle number for the $\mathrm{Ca}: \mathrm{TiO}_{2}-\mathrm{B}-\mathrm{LiPF}_{6}-\mathrm{LiFePO}_{4}$ battery at the rate of (a) $20 \mathrm{C}$, and (b) $40 \mathrm{C}\left(1 \mathrm{C}=294 \mathrm{~mA} \mathrm{~g}^{-1}\right)$. The voltage window is $0.5-2 \mathrm{~V}$. 147

Figure 6.4 Long term capacity retention of the $\mathrm{Ca}: \mathrm{TiO}_{2}-\mathrm{B}-\mathrm{LiPF}_{6}-\mathrm{LiFePO}_{4}$ battery at a $80 \mathrm{C}$ rate $\left(1 \mathrm{C}=294 \mathrm{~mA} \mathrm{~g}^{-1}\right)$. The voltage window is $0.5-2 \mathrm{~V}$.

Figure 6.5 Electrochemical voltage profiles at different cycles between $0.5 \mathrm{~V}$ and $2 \mathrm{~V}$ for the $\mathrm{Ca}: \mathrm{TiO}_{2}-\mathrm{B}-\mathrm{LiPF}_{6}-\mathrm{LiFePO}_{4}$ battery at a $10 \mathrm{C}\left(1 \mathrm{C}=294 \mathrm{~mA} \mathrm{~g}^{-1}\right)$ rate based on $\mathrm{Ca}: \mathrm{TiO}_{2}-\mathrm{B}$.

Figure 6.6 Charge-discharge voltage profiles of the $\mathrm{LiMn}_{2} \mathrm{O}_{4}$ slurry electrode vs. Li counter electrode at $1 \mathrm{C}$ rate from the 1st cycle to the 150th cycle. [Figure courtesy: Frances Venable and Prof. Bart M. Bartlett] 150

Figure 6.7 Charge and discharge capacities vs. cycle number for the $\mathrm{Ca}: \mathrm{TiO}_{2}-\mathrm{B}-\mathrm{LiPF}_{6}-\mathrm{LiMn}_{2} \mathrm{O}_{4}$ battery at the rate of (a) $20 \mathrm{C}$, and (b) $40 \mathrm{C}\left(1 \mathrm{C}=294 \mathrm{~mA} \mathrm{~g}^{-1}\right)$. The voltage window is 1.2-3 V. . 151

Figure 6.8 Long term capacity retention of the $\mathrm{Ca}: \mathrm{TiO}_{2}-\mathrm{B}-\mathrm{LiPF}_{6}-\mathrm{LiMn}_{2} \mathrm{O}_{4}$ battery at a $80 \mathrm{C}$ rate $\left(1 \mathrm{C}=294 \mathrm{~mA} \mathrm{~g}^{-1}\right)$. The voltage window is $1.2-3 \mathrm{~V}$. 152

Figure 6.9 Electrochemical voltage profiles at different cycles between $1.2 \mathrm{~V}$ and $2.7 \mathrm{~V}$ for the $\mathrm{Ca}: \mathrm{TiO}_{2}-\mathrm{B}-\mathrm{LiPF}_{6}-\mathrm{LiMn}_{2} \mathrm{O}_{4}$ battery at a $10 \mathrm{C}\left(1 \mathrm{C}=294 \mathrm{~mA} \mathrm{~g}^{-1}\right)$ rate based on Ca:TiO $2-\mathrm{B}$. 153

Figure A.1 (Left) band structure of the $\mathrm{TiO}_{2}-\mathrm{B}$ polymorph with valence band maximum located at $0 \mathrm{eV}$. (Right) schematic of the Brillouin zone with several high-symmetry points labeled. [Figure courtesy: Dylan Bayerl and Prof. Emmanouil Kioupakis].

Figure B.1 a) STEM image of a $\mathrm{SrTi}_{0.75} \mathrm{Nb}_{0.20} \mathrm{Pt}_{0.05} \mathrm{O}_{3}$ film grown in $0.01 \mathrm{mT} \mathrm{O}_{2}$ at $800{ }^{\circ} \mathrm{C}$ containing Pt nanoparticles as-grown. b) High resolution HAADF STEM image showing one Pt particle with $\sim 2.5 \mathrm{~nm}$ diameter in the STO host. c) A typical Pt particle sitting on the surface of the film. [TEM image credit: Shuyi Zhang] 173

Figure B.2 Comparison of electrical properties in four STNPO films growing in either 20 mTorr Ar or $0.01 \mathrm{mTorr}_{2}$, all containing 5\% Pt which has precipitated as metallic nanoparticles: a) carrier concentration; b) electrical conductivity. [Temperature dependent transport data measured by Hang Chi in Prof. Ctirad Uher's group at Department of Physics] . 174

Figure B.3 a) HAADF STEM image of an elongated Pt nanoparticle. b) EDS spectrum taken as the beam was focused on the Pt particle. c) STEM image of a $\mathrm{SrTi}_{0.65} \mathrm{Nb}_{0.30} \mathrm{Pt}_{0.05} \mathrm{O}_{3}$ film grown in Ar atmosphere showing Pt nanorods. [TEM image credit: Shuyi Zhang \& Yi Zhang]..... 175 
Figure B.4 Temperature dependent electronic transport properties in two $30 \% \mathrm{Nb}$ doped films grown in Ar atmosphere, with or without Pt nano-inclusions: a) electrical conductivity; b) Hall mobility; c) carrier concentration; d) Seebeck coefficient. [Temperature dependent transport data measured by Hang Chi in Prof. Ctirad Uher's group at Department of Physics]...................... 176

Figure B.5 Temperature dependent power factor in four 30\% Nb doped STNPO films. At $300 \mathrm{~K}$, the film containing Pt nanorods grown in Ar shows a 3.5 $\times$ increase of $P F$ from the film with no Pt grown in $\mathrm{O}_{2}$ 177 


\section{List of Appendices}

Appendix A

$\mathrm{TiO}_{2}$-B Band Structure by First Principles Calculations .............................................. 163

Appendix B

Enhanced Thermoelectric Properties in a Metal-Perovskite System ................................ 166 


\section{Abstract \\ Epitaxial $\mathrm{CaTi}_{5} \mathrm{O}_{11}$ and $\mathrm{TiO}_{2}$-B Thin Films for High Rate Lithium-Ion Batteries \\ by \\ Kui Zhang}

Chair: Xiaoqing Pan

The bronze polymorph of titanium dioxide $\left(\mathrm{TiO}_{2}-\mathrm{B}\right)$ is interesting for many applications including high rate energy storage, solar cells, photocatalysis, thermoelectrics and sensing, owing to its uniquely layered structure and highly asymmetric unit cell. However, such a metastable phase is extremely hard to obtain with high purity and crystallinity, significantly impeding its development in these fields. This dissertation is devoted to the waterless synthesis, structural characterization and property testing of both $\mathrm{TiO}_{2}-\mathrm{B}$ and a related novel material, $\mathrm{CaTi}_{5} \mathrm{O}_{11}$, in the form of highly crystalline thin films, with a specific emphasis on their application as anode materials in lithium-ion batteries (LIBs).

Although known to have advantages over anatase or rutile, high quality bronze phase $\mathrm{TiO}_{2}-\mathrm{B}$ specimens that demonstrate good electrochemical properties thus far have exclusively been nano-structured powders prepared by hydrothermal methods, as first synthesized in 1980 . Aided by first-principles calculation and atomic resolution high-angle annular dark-field (HAADF) scanning transmission electron microscopy (STEM), it has been discovered that $\mathrm{Ca}$ 
can stabilize the bronze structure, forming a variant phase $\mathrm{CaTi}_{5} \mathrm{O}_{11}$, which has then been successfully synthesized in epitaxial single-crystalline thin films by pulsed laser deposition (PLD), a completely waterless process. Due to the near-perfect lattice match, the $\mathrm{CaTi}_{5} \mathrm{O}_{11}$ film can be further used as a template layer to grow high quality, water-free $\mathrm{TiO}_{2}-\mathrm{B}$ films on top, which facilitates the synthesis and application of both materials on a wide variety of substrates, including $\mathrm{SrTiO}_{3}, \mathrm{Nb}: \mathrm{SrTiO}_{3}, \mathrm{LaAlO}_{3}$, LSAT and $\mathrm{SrTiO}_{3}$ buffered Si.

Lithium ion transport in the bronze structure is highly anisotropic. By utilizing substrates with a different orientation to align the more open channels with out-of-plane directions, extremely high rates of lithium ion transport, up to $600 \mathrm{C}\left(1 \mathrm{C}=335 \mathrm{~mA} \mathrm{~g}^{-1}\right)$, with extraordinary structural stability has been achieved. In a battery half-cell using metallic lithium as counter electrode, the orientation-engineered $\mathrm{CaTi}_{5} \mathrm{O}_{11}$ film discharged to $155 \mathrm{~mA} \mathrm{~h} \mathrm{~g}^{-1}$ at a rate of $60 \mathrm{C}$, corresponding to a time of $60 \mathrm{~s}$ to fully discharge the capacity, at the 100th cycle, delivering specific power of $\sim 20 \mathrm{~kW} \mathrm{~kg}{ }^{-1}$. Post-mortem examinations by x-ray diffraction (XRD) and transmission electron microscopy (TEM) confirmed that both the $\mathrm{TiO}_{2}-\mathrm{B}$ and $\mathrm{CaTi}_{5} \mathrm{O}_{11}$ structures were essentially unchanged after aggressively cycling for more than 60 days.

The results discussed in this thesis provide the basic knowledge needed to realize and utilize $\mathrm{TiO}_{2}-\mathrm{B}$ single crystals, while also supporting theoretical studies with determinate experimental data. The ability to accurately control the crystal orientation will be especially beneficial to studies focused on surface states, such as in photocatalysis and photovoltaic applications. As the methods and equipment required are readily accessible to the extended research community, further studies on and applications of these materials, which are attractive in realms that extend beyond electrochemistry, may emerge. 


\section{Chapter 1}

\section{Introduction}

\subsection{An overview of the $\mathrm{TiO}_{2}$ material}

Titanium dioxide $\left(\mathrm{TiO}_{2}\right)$ is a wide band-gap $(3.0-3.2 \mathrm{eV})$ semiconductor and a focus of attention for many applications, including solar energy conversion, lithium-ion battery, thermoelectrics, photocatalysis, water splitting and sensors. ${ }^{1-5} \mathrm{TiO}_{2}$ generally exists in nature as three polymorphs - rutile, anatase and brookite. The bronze polymorph, or $\mathrm{TiO}_{2}-\mathrm{B}$, was recently found in nature in Valais, Switzerland. ${ }^{6}$ Additionally, $\mathrm{TiO}_{2}$ may adopt four other crystal structures, including $\mathrm{TiO}_{2}$-II (“columbite"), $\mathrm{TiO}_{2}$-III ("baddeleyite"), $\mathrm{TiO}_{2}-\mathrm{H}$ ("hollandite") and $\mathrm{TiO}_{2}-\mathrm{R}$ ("ramsdellite"). Among them, $\mathrm{TiO}_{2}$-II and -III were synthesized by a high-pressure treatment of anatase or rutile, while $\mathrm{TiO}_{2}-\mathrm{H}$ and $-\mathrm{R}$ were synthesized by topotactic oxidative extraction of alkali metal from $\mathrm{K}_{0.25} \mathrm{TiO}_{2}$ (hollandite) and $\mathrm{Li}_{0.5} \mathrm{TiO}_{2}$, respectively. Structure parameters of these distinctive polymorphs are listed Table 1.1.

Although its commercial production started in the early twentieth century, often found in pigments, sunscreens, paints, ointments, toothpaste, etc., research efforts on the $\mathrm{TiO}_{2}$ material essentially began to surge since 1972, when Fujishima and Honda discovered the phenomenon of photocatalytic water splitting on a $\mathrm{TiO}_{2}$ electrode under ultraviolet (UV) light. ${ }^{1,3}$ Since then, tens of thousands of reports have been published on this material, especially focused on applications spread over energy and environmental fields - the top two conundrums the world faces today. Utilizing $\mathrm{TiO}_{2}$ for various scientific and technical purposes often depend not only on the properties of the material itself, but also on the tuning and modification of the $\mathrm{TiO}_{2}$ host, often 
via doping, alloying and nano-structuring.

\subsection{The structures of $\mathrm{TiO}_{2}-\mathrm{B}$ and $\mathrm{Ca}: \mathrm{TiO}_{2}-\mathrm{B}\left(\mathrm{CaTi}_{5} \mathrm{O}_{11}\right)$}

$\mathrm{TiO}_{2}$-B was first synthesized by Marchand et al. in 1980 by ion exchange of $\mathrm{K}^{+}$for $\mathrm{H}^{+}$in $\mathrm{K}_{2} \mathrm{Ti}_{4} \mathrm{O}_{9}$ in a hydrothermal process. ${ }^{7}$ Similar to rutile and anatase, $\mathrm{TiO}_{2}-\mathrm{B}$ is composed of corrugated sheets of edge- and corner-sharing $\mathrm{TiO}_{6}$ octahedra, but the sheets are joined together to form a three-dimensional framework, ${ }^{8}$ forming perovskite-like pathways which potentially facilitates facile transport for $\mathrm{Li}^{+}$into the bulk of the crystal. The $\mathrm{TiO}_{2}-\mathrm{B}$ structure projected along three crystallographic directions is shown in Figure 1.1 (top row).

The $\mathrm{CaTi}_{5} \mathrm{O}_{11}$ phase was first identified as a defect phase inclusion in $\mathrm{CaTiO}_{3}$ thin films grown by PLD on $\mathrm{SrTiO}_{3}$ substrates. Systematic optimization in PLD growth has been carried out to synthesize this novel phase into highly crystalline thin films with superior purity, which were then characterized by atomic resolution HAADF STEM. According to the STEM images, we have built the geometric model and then performed first-principles optimization by PW91 functional implemented in $\operatorname{VASP}^{[9,10]}$. Our more accurate HSE06 method ${ }^{[11]}$ calculations indicate that $\mathrm{CaTi}_{5} \mathrm{O}_{11}$ (stoichiometry), or $\mathrm{Ca}_{4} \mathrm{Ti}_{20} \mathrm{O}_{44}$ in a unit cell, is an orthorhombic structure with lattice constants of $a=12.1702 \AA, b=3.8013 \AA, c=17.9841 \AA, \quad \alpha=\beta=\gamma=90^{\circ}$. The atom positions are listed in Table 1.2. Different methods used in the first-principle calculations to relax the structure may result in slightly different lattice parameters as well as the symmetry associated, but the difference is usually too small to be observed experimentally. In our current working model, the $\mathrm{CaTi}_{5} \mathrm{O}_{11}$ phase has the symmetry of CMCM (space group no. 63).

The structure of the $\mathrm{CaTi}_{5} \mathrm{O}_{11}$ phase is closely related to the $\mathrm{TiO}_{2}-\mathrm{B}$ polymorph, essentially comprising repeatedly twinned $a-b$ plates of $\mathrm{TiO}_{2}-\mathrm{B}$ interleaved with one alternating $\mathrm{Ti}-\mathrm{Ca}$ ion layer between every two layers of $\mathrm{Ti}$ ions, turning the regular $\mathrm{TiO}_{2}-\mathrm{B}$ structure into a zigzag pattern. Such a structure is compared with $\mathrm{TiO}_{2}$-B in Figure 1.1 (bottom row). Therefore, the $\mathrm{CaTi}_{5} \mathrm{O}_{11}$ phase is essentially a variant of the $\mathrm{TiO}_{2}-\mathrm{B}$ structure with extra $\mathrm{Ca}$ layers and superlattice twinning, and we designate the new phase as $\mathrm{Ca}: \mathrm{TiO}_{2}-\mathrm{B}$, which is repeatedly used 
throughout this thesis.

We discovered the new $\mathrm{CaTi}_{5} \mathrm{O}_{11}$ phase on an entirely independent basis. It was at the very last stage of publishing this discovery ${ }^{12}$ when we noticed a patent application published in 2011 mentioning such a phase for different uses by an Italian cement company. ${ }^{13}$ Nevertheless, we provide the first clear depiction of this phase on an atomic scale, as well as the first waterless process to synthesize it with high quality and purity, which in turn benefit a clear interpretation of electrochemical testing data. There is no other report on this phase in the literature.

By visual inspection (Figure 1.1), the channels running along the $b$-axis appear to be most open among the three crystallographic axes in both two structures, and may be a good candidate for high $\mathrm{Li}^{+}$mobility in the crystal. Channels parallel to various other directions can also be found by manipulating the model. It is worth noting that more rigorous study than simply observing the cross sectional areas of the channel opening is required to determine with relative certainty the actual diffusion path that is most energetically favorable for fast $\mathrm{Li}^{+}$transport, ${ }^{14}$ as the results may be counter-intuitive. The ability to fabricate crystalline thin films of the active storage material with well-defined lattice plane on the surface, such as what will be demonstrated in this thesis, is therefore of great value to experimentally determine the preferred $\mathrm{Li}^{+}$pathways.

\section{3 $\mathrm{TiO}_{2}-\mathrm{B}$ in lithium-ion batteries}

Energy storage materials with high capacity and rapid charge/discharge rates hold the key to the next breakthrough in lithium-ion batteries (LIBs), expanding the application to high power systems such as electric vehicles (EVs) and smart grids. ${ }^{15}$ On the commercial level, the ability to charge and discharge batteries in the matter of seconds, instead of hours, will induce lifestyle changes, e.g. to fully charge a smartphone in two minutes, or to charge an electric car in the time it usually takes to fill up the tank of an ICE (internal combustion engine) vehicle at the gas station. High rate discharge is also essential to enhance the performance of EVs, where a powerful acceleration may often be required.

$\mathrm{TiO}_{2}-\mathrm{B}$ is an excellent candidate in this regard due to its open structure ${ }^{7}$ and fast lithium 
ion transport via a pseudocapacitive Faradaic process, leveraging ultrahigh discharge rates comparable to those of supercapacitors while maintaining the advantage of storing energy in the bulk. ${ }^{16,17}$ However, the existing powder/slurry form prepared by hydrothermal methods ${ }^{18-25}$ cannot provide clear insight into its fundamental characteristics, due to the limited purity, the randomized crystal orientation and the unavoidable presence of lattice water in its structure. ${ }^{26}$ This thesis discusses in great detail the discovery and optimization of a waterless process to synthesize hetero-epitaxial crystalline thin films of $\mathrm{TiO}_{2}-\mathrm{B}$ using PLD onto $\mathrm{Ca}: \mathrm{TiO}_{2}-\mathrm{B}$, which serves as a template.

$\mathrm{TiO}_{2}$ has been extensively investigated as an anode material for the LIB due to its low cost, minimal environmental impact, structural stability, high theoretical capacity $\left(335 \mathrm{~mA} \mathrm{~h} \mathrm{~g}^{-1}\right)$ and inherent safety (a buffer $>1.5 \mathrm{~V}$ before lithium plating). ${ }^{27,28}$ Fast lithium storage has been demonstrated in anatase, rutile and $\mathrm{Li}_{4} \mathrm{Ti}_{5} \mathrm{O}_{12}$ nanostructures. ${ }^{29-32}$ Although known to have advantages over anatase or rutile, high quality bronze phase $\mathrm{TiO}_{2}-\mathrm{B}$ specimens that demonstrate good electrochemical properties thus far have exclusively been nano-structured powders prepared by hydrothermal methods, ${ }^{23,24,26,33-36}$ as first synthesized in $1980 .{ }^{7}$ Being a metastable phase, compounded by the fact that $\mathrm{TiO}_{2}$-anatase which rarely fully reacts ${ }^{37}$ is often used as a precursor in existing synthesis methods, phase pure $\mathrm{TiO}_{2}-\mathrm{B}$ has been extremely difficult to obtain, obscuring the interpretation of property testing results. In addition, removal of all $\mathrm{H}_{2} \mathrm{O}$, which could interfere with $\mathrm{Li}^{+}$transport, from the final product proves to be difficult, and recent studies have suggested that $\mathrm{H}_{2} \mathrm{O}$ may even be needed to keep it from collapsing into anatase upon aggressive heating. ${ }^{26,38,39}$

\subsection{Objectives and organization of this dissertation}

In light of the above, the objectives of this dissertation are: (1) to synthesize high quality epitaxial thin films of both $\mathrm{TiO}_{2}-\mathrm{B}$ and $\mathrm{Ca}: \mathrm{TiO}_{2}-\mathrm{B}$ on various substrates; (2) to characterize the microstructures of both materials and the interfaces between them, as well as the interface and epitaxial relationship between the thin films and the substrates; (3) to devise a configuration in 
which the thin films can be assembled in a battery half-cell, and a testing protocol to ensure the accuracy of recorded data for analysis; (4) to study the electrochemical properties of these bronze thin films; and (5) to enhance the battery performance by manipulating the crystal structure of the thin films.

This dissertation is organized as follows: Chapter 2 explains the experimental methods including pulsed laser deposition and electrochemical property testing. Chapter 3 provides a detailed description of the systematic study on the new, waterless synthesis route to fabricate single-crystalline epitaxial thin films of both $\mathrm{TiO}_{2}-\mathrm{B}$ and $\mathrm{Ca}: \mathrm{TiO}_{2}-\mathrm{B}$. The optimization of the PLD growth conditions, including target composition, choice of substrates, growth temperature, laser energy and $\mathrm{O}_{2}$ partial pressure to achieve the best purity and crystallinity, as well as a templating effect between the two phases, is discussed. Chapter 4 presents the effort to test these bronze thin films as anode materials in a battery half-cell, including experimental setup and data collection protocol. This process is important and creative for measuring such a tiny mass of active material, which is rarely seen in this field of study and also instructive for future research on this topic. The structural stability and electrochemical properties of the thin films, including battery cycling and capacity retention, are explored. In Chapter 5, significant enhancement in battery performance is achieved by changing the crystal structure using substrates with a different orientation, presenting superior high rate capabilities in polycrystalline $\mathrm{Ca}: \mathrm{TiO}_{2}-\mathrm{B}$ thin films. The microstructures in the film and at the film-substrate interface are investigated, and further related to the underlying physics of the material's behavior. The battery cycling results of prototype full-cell batteries featuring the polycrystalline $\mathrm{Ca}: \mathrm{TiO}_{2}-\mathrm{B}$ thin film anode and $\mathrm{LiFePO}_{4}$ or $\mathrm{LiMn}_{2} \mathrm{O}_{4}$ slurry cathodes are exhibited in Chapter 6. Finally, conclusions drawn from current results and proposals for future work in Chapter 7 conclude the thesis. 
Table 1.1 Structural parameters of $\mathrm{TiO}_{2}$ polymorphs, summarized from Ref. 27 and Ref. 40.

\begin{tabular}{llll}
\hline Phase & Space group & $\begin{array}{l}\text { Density } \\
\left(\mathrm{g} \mathrm{cm}^{-3}\right)\end{array}$ & Lattice parameters \\
\hline Anatase & I4 $4_{1} /$ amd & 3.79 & $a=3.78 \AA, c=9.51 \AA$ \\
Rutile & $P 4_{2} / m n m$ & 4.13 & $a=4.59 \AA, c=2.96 \AA$ \\
Brookite & $P b c a$ & 3.99 & $a=9.17 \AA, b=5.46 \AA, c=5.14 \AA$ \\
$\mathrm{TiO}_{2}$-B & $C 2 / m$ & 3.62 & $a=12.18 \AA, b=3.74 \AA, c=6.52 \AA, \beta=107.05^{\circ}$ \\
$\mathrm{TiO}_{2}$-II & $P b c n$ & 4.33 & $a=4.52 \AA, b=5.5 \AA, c=4.94 \AA$ \\
$\mathrm{TiO}_{2}$-III & $P 2_{1} / c$ & 3.79 & $a=4.64 \AA, b=4.76 \AA, c=4.81 \AA, \beta=99.2^{\circ}$ \\
$\mathrm{TiO}_{2}$ - $\mathrm{H}$ & $I 4 / m$ & 3.46 & $a=10.18 \AA, c=2.97 \AA$ \\
$\mathrm{TiO}_{2}-\mathrm{R}$ & $P b n m$ & 3.87 & $a=4.9 \AA, b=9.46 \AA, c=2.96 \AA$ \\
\hline
\end{tabular}


Table 1.2 Atom positions in the $\mathrm{CaTi}_{5} \mathrm{O}_{11}$ structure.

\begin{tabular}{|c|c|c|c|c|c|c|c|c|c|c|c|}
\hline$\#$ & \multicolumn{2}{|c|}{ Atom } & \multirow{2}{*}{$\frac{\boldsymbol{x}}{0.19999}$} & \multirow{2}{*}{$\begin{array}{c}y \\
0.93727\end{array}$} & \multirow{2}{*}{$\begin{array}{c}\boldsymbol{Z} \\
0.34267\end{array}$} & \multirow{2}{*}{$\frac{\#}{35}$} & \multicolumn{2}{|c|}{ Atom } & \multirow{2}{*}{$\begin{array}{c}\boldsymbol{x} \\
0.44389\end{array}$} & \multirow{2}{*}{$\begin{array}{c}y \\
0.43501\end{array}$} & \multirow{2}{*}{$\frac{\boldsymbol{Z}}{0.48876}$} \\
\hline 1 & $\mathrm{O}$ & $\mathrm{O} 1$ & & & & & $\mathrm{O}$ & O35 & & & \\
\hline 2 & $\mathrm{O}$ & $\mathrm{O} 2$ & 0.04096 & 0.93438 & 0.98907 & 36 & $\mathrm{O}$ & & 0.19931 & 0.93896 & 0.49221 \\
\hline 3 & $\mathrm{O}$ & & & & & & $\mathrm{O}$ & & & & .84527 \\
\hline 4 & $\mathrm{O}$ & $\mathrm{O} 4$ & 7177 & .93646 & .21254 & 38 & $\mathrm{O}$ & & 28431 & 43673 & 0.8422 \\
\hline 5 & $\mathrm{O}$ & O5 & & & & 39 & $\mathrm{O}$ & & 03923 & 93309 & .84518 \\
\hline 6 & $\mathrm{O}$ & O6 & 0.7005 & & 0.34213 & 40 & $\mathrm{O}$ & & 0.78454 & 0.93558 & 0.84229 \\
\hline 7 & $\mathrm{O}$ & O7 & & 0.43719 & 0.98906 & 41 & $\mathrm{O}$ & & 0.5666 & 0.93536 & 0.41677 \\
\hline 8 & $\mathrm{O}$ & O8 & & & & 4 & $\mathrm{O}$ & & & .93805 & .91639 \\
\hline 9 & $\mathrm{O}$ & O9 & 57158 & 0.43 & 2121 & 43 & $\mathrm{O}$ & 43 & 0.91796 & 0.43314 & 0.91749 \\
\hline 10 & $\mathrm{O}$ & $\mathrm{O} 10$ & 0.88567 & & & & $\mathrm{O}$ & & 0.06658 & 0.43879 & 0.41808 \\
\hline 11 & $\mathrm{O}$ & & & & & 45 & $\mathrm{Ti}$ & & & & \\
\hline 12 & $\mathrm{O}$ & $\mathrm{O} 12$ & 0.94481 & 0.93819 & 0.34546 & 46 & $\mathrm{Ti}$ & & 0.06116 & .93641 & 0.09019 \\
\hline 13 & $\mathrm{O}$ & $\mathrm{O} 13$ & 0.75278 & 0.93744 & 0.22097 & 4 & $\mathrm{Ti}$ & Ti3 & 0.72074 & 0.43715 & 0.24147 \\
\hline 14 & $\mathrm{O}$ & & 0.91413 & & & 40 & $\mathrm{Ti}$ & & & & 0.09009 \\
\hline 15 & $\mathrm{O}$ & & & & & & $\mathrm{Ti}$ & & & & \\
\hline 16 & $\mathrm{O}$ & O16 & 0.28498 & 0.43691 & 0.9919 & 50 & $\mathrm{Ti}$ & i6 & 0.92466 & 0.93757 & 0.2442 \\
\hline 17 & $\mathrm{O}$ & O17 & 0.44504 & 0.43607 & 0.34505 & 51 & $\mathrm{Ti}$ & i7 & 0.26493 & 0.43673 & 0.09246 \\
\hline 18 & $\mathrm{O}$ & & & & & 52 & $\mathrm{Ti}$ & & & & \\
\hline 19 & $\mathrm{O}$ & & & & & & $\mathrm{Ti}$ & & & & 0.59265 \\
\hline 20 & $\mathrm{O}$ & $\mathrm{O} 20$ & 0.09999 & 0.43618 & 0.0913 & 5 & $\mathrm{Ti}$ & Ti10 & 0.06009 & 0.93443 & 0.74408 \\
\hline 21 & $\mathrm{O}$ & $\mathrm{O} 21$ & 0.23209 & 0.93441 & 0.72099 & 55 & $\mathrm{Ti}$ & Ti11 & 0.71913 & 43538 & 0.59214 \\
\hline 22 & $\mathrm{O}$ & & 0.07037 & & 0.62223 & & $\mathrm{Ti}$ & & 0.56028 & & 0.74408 \\
\hline 23 & $\mathrm{O}$ & & & & & & $\mathrm{Ti}$ & & 0.26434 & & 0.7417 \\
\hline 24 & $\mathrm{O}$ & $\mathrm{O} 24$ & 0.73211 & 0.43547 & 0.72103 & 58 & $\mathrm{Ti}$ & Ti14 & 0.42346 & 0.43479 & 0.58992 \\
\hline 25 & $\mathrm{O}$ & & & & & 59 & $\mathrm{Ti}$ & & & & \\
\hline 26 & $\mathrm{O}$ & $\mathrm{O} 26$ & 0.88417 & & & & $\mathrm{Ti}$ & & 0.9231 & 0.93527 & 0.59028 \\
\hline 27 & $\mathrm{O}$ & $\mathrm{O} 27$ & 0.75136 & 0.93542 & 0.61322 & & $\mathrm{Ti}$ & & 0.58362 & 0.45134 & 0.41619 \\
\hline 28 & $\mathrm{O}$ & $\mathrm{O} 28$ & 0.2515 & 0.43522 & 0.61321 & 62 & $\mathrm{Ti}$ & Ti18 & 0.40087 & 0.42763 & 0.91679 \\
\hline 29 & $\mathrm{O}$ & $\mathrm{O} 29$ & 0.41305 & 0.43458 & 0.71227 & 63 & $\mathrm{Ti}$ & Ti19 & 0.90097 & 0.9446 & 0.91686 \\
\hline 30 & $\mathrm{O}$ & $\mathrm{O} 30$ & 0.0992 & 0.43408 & 0.74361 & 64 & $\mathrm{Ti}$ & Ti20 & 0.08325 & 0.93465 & 0.41732 \\
\hline 31 & $\mathrm{O}$ & $\mathrm{O} 31$ & 0.91301 & 0.93537 & 0.71234 & (0) & $\mathrm{Ca}$ & & 0.87195 & 0.43614 & 0.41755 \\
\hline 32 & $\mathrm{O}$ & $\mathrm{O} 32$ & & 0.93577 & & 66 & $\mathrm{Ca}$ & & & & 0.41769 \\
\hline 33 & $\mathrm{O}$ & $\mathrm{O} 33$ & 0.94383 & & & 67 & $\mathrm{Ca}$ & & 0.61261 & 0.93498 & 0.91722 \\
\hline 34 & $\mathrm{O}$ & O34 & 0.69927 & 0.43558 & 0.49164 & 68 & $\mathrm{Ca}$ & $\mathrm{Ca} 4$ & 0.11262 & 0.43539 & 0.91721 \\
\hline
\end{tabular}




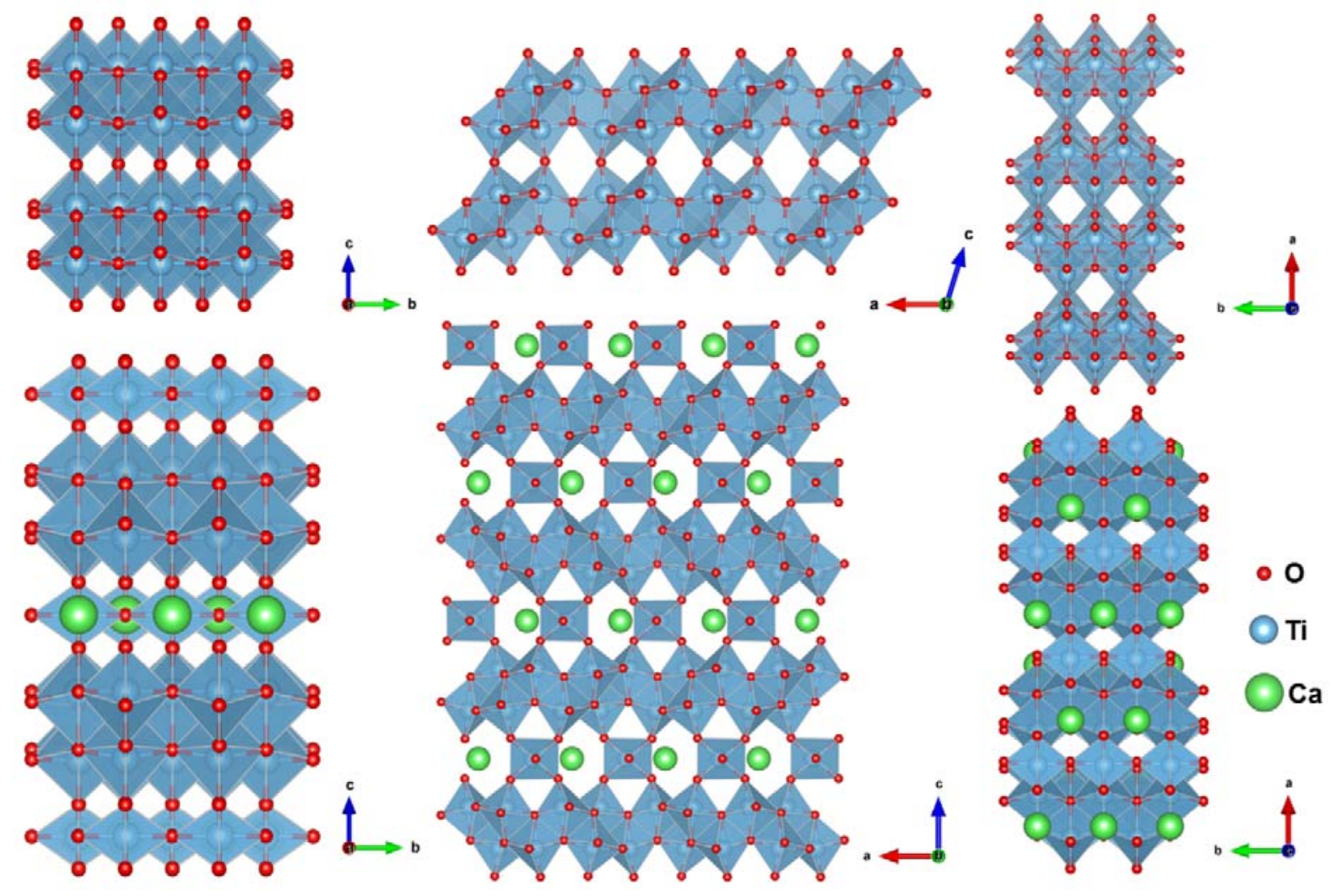

Figure 1.1 Crystal structures of regular $\mathrm{TiO}_{2}-\mathrm{B}$ (top row) and $\mathrm{Ca}: \mathrm{TiO}_{2}-\mathrm{B}\left(\mathrm{CaTi}_{5} \mathrm{O}_{11}\right.$, bottom row) projected along [100], [010] and [001] directions, respectively. These drawings were produced using VESTA. 


\section{Chapter 2 \\ Experimental Methods}

\subsection{Epitaxial growth by pulsed laser deposition}

Epitaxy refers to the deposition of a crystalline overlayer on a crystalline substrate with single, well defined, related orientation relationships. Atom bonds usually form at the interface between the film and the substrate.

All thin films or epilayers studied in this work were fabricated by pulsed laser deposition, or PLD for short.

\subsubsection{An overview of the PLD technique}

The PLD method of crystalline thin film growth consists of three steps: (1) the interaction of the laser beam with the target, which is usually either a ceramic target in the form of a sintered pellet, or a bulk single crystal (ablation); (2) plasma formation, heating, and initial three-dimensional isothermal expansion (often called a 'plume'), and (3) adiabatic expansion and deposition of thin films on the surface of the substrate, which is often maintained at a certain growth temperature. ${ }^{41}$

PLD is one of the most versatile methods to grow ceramic and metallic thin films. Doping is especially convenient in such technique, as the select elements are simply mixed in the powder form with calculated mole percentage, which is then sintered at high temperatures. Hence, PLD is a very efficient method to synthesize functional thin films, which are often times doped or off-stoichiometry, such as superconductor, ${ }^{42}$ semiconductor ${ }^{43,44}$ and ferroelectric 
films. $^{45}$

During growth, a high energy pulsed laser beam (commonly from excimer, $\mathrm{CO}_{2}$ or Nd:YAG lasers) is focused onto the surface of the solid target. The strong electromagnetic radiation leads to rapid evaporation of the target material, exciting ionized species that are presented as a glowing plasma plume in front of the target surface, the color of which varies depending on the target composition, if the ablation is carried out in vacuum or low pressure ambient. As the pressure increases, the energized particles are quickly scattered by the gas molecules, resulting in a shorter mean-free path and consequently a weaker plume.

The most advantageous merit of PLD over other common growth techniques is that it allows a direct transfer of stoichiometry from the target to the film, which crystallizes in the most energetically favorable phase that matches the surface structure of the substrate under the specific thermodynamic growth environment, independent of the phase of the target, as long as it has the desired stoichiometry. Therefore, PLD provides rapid growth of almost any material over a wide range of deposition conditions, including target composition, choice of substrate, growth temperature, laser energy (fluence), laser pulse repetition rate and gas ambient (in partial pressure). The major components of a PLD system are a laser, optics, and a vacuum chamber equipped with pumps, a substrate holder/heater and a target controller. Many accessories are commercially available to expand the system capabilities, such as reflection high-energy electron diffraction (RHEED) source and detector which enables in situ monitoring of the film surface during deposition.

A typical PLD system is shown schematically in Figure 2.1. While the high energy laser pulses will easily drill a hole on the target surface if it sits still, many systems have a multi-target carousel equipped with motors which rotate and raster the selected target, so that the laser uniformly ablate the target surface to maximize utilization rate of the target material, as well as to ensure that the excited plume is directed toward the substrate, since the plume is always perpendicular to the local surface plane where the laser hits the target. For systems without the capability to rotate and raster the targets, an oscillating mirror mounted on a stepper motor (as 
shown in Figure 2.1) is often installed which makes the laser beam scan the target surface.

The major issue with the PLD technique is the purity of the target. While doping in the ceramic target is convenient, the introduction of unexpected elements is difficult to eliminate in the meantime. Since the target making process usually starts with mixing chemical powders, followed by uniaxial or isostatic pressing and sintering, contamination is also possible. The unintentional impurities, if transferred into the deposited films, may cause undesired effects such as additional conducting carriers, extra structural defects or excitonic broadening.

Another limitation of the PLD method is its relatively small yield. The laser plume is a cone-shaped plasma which has a larger ion concentration in the center. Therefore, the deposited films are usually thicker in the area that is directionally aligned with the center of the plume. While such effect may not be significant for small substrates, it will be quite obvious when the substrate is larger than $10 \times 10 \mathrm{~mm}^{2}$. Because different ions travel at different speeds from the target to the substrate, for even larger substrates, e.g. wafers with a diameter of 3 " or above, it is very difficult to maintain uniform coverage, layer composition and thickness. Therefore, PLD is not a suitable technique for scaling up production, in which case sputtering or various chemical vapor deposition (CVD) methods would be more efficient. Nevertheless, the growth mechanism and conditions obtained from PLD research are valuable guidelines when transferring to other depositions platforms.

\subsubsection{Growth conditions in the PLD process}

There are seven main parameters that can be tuned to optimize the deposition process and to achieve high quality or specially designed thin films in the PLD method: target composition, choice of substrates, target-substrate distance, growth temperature, laser fluence, laser repetition rate, and gas backfill pressure.

(1) Target composition:

As the starting point of the PLD process, the target composition determines, to a large extent, the final outcome of the film structure and properties. For common compounds such as 
$\mathrm{ZnO}$ and $\mathrm{SrTiO}_{3}$, the only concern is the purity, and these targets are often commercially available. It becomes much more complicated when designing a new material. For example, $\mathrm{SrTiO}_{3}$ could be co-doped with $\mathrm{Nb}$ and $\mathrm{Pt}$, where $\mathrm{Nb}$ substitutes some $\mathrm{Ti}$ ions to increase electrical conductivity, while Pt may precipitate as nano-particles embedded in the $\mathrm{SrTiO}_{3}$ matrix, which helps reduce the thermal conductivity (see Appendix B). Not only the percentages of the dopants need to be carefully measured, the mixing, pressing and sintering recipes also require fine tuning because the source materials $\left(\mathrm{SrTiO}_{3}, \mathrm{Nb}_{2} \mathrm{O}_{5}\right.$ and $\mathrm{PtO}_{2}$ powders in this case) have different physical properties including melting point and compressibility. A uniform distribution of the species in the final sintered pellet, usually very dense to avoid cracking upon laser ablation, is crucial to the film's phase forming and long-term repeatability in the research project.

In many cases, the phase the thin film crystallizes in is sensitive to the composition of the target. As will be elaborated in Chapter 3 of this thesis, the phase purity of the $\mathrm{Ca}: \mathrm{TiO}_{2}-\mathrm{B}$ films depends heavily on the Ca content in the target. Since target making is a time-consuming task, theoretical guidance is of great value in exploring new phases and novel materials than simply trying out many different compositions. In this thesis, the optimization of bronze film growth benefited extensively from the phase diagrams deduced by first principles calculations.

While the laser pulses ablate the target, unique phenomena such as melting, ripple pattern formation, ${ }^{46}$ and phase segregation are usually observed on the target surface. The amount of material ablated per pulse is deducted from the heat balance equation: ${ }^{41}$

$$
\Delta x_{t}=\frac{(1-R)\left(E-E_{t h}\right)}{\Delta H+C_{v} \Delta T}
$$

where $\Delta x_{t}$ is the evaporated thickness of the target, $R$ is the reflectivity of the target material, $E_{t h}$ is the energy threshold above which evaporation occurs, $\Delta H$ is the volume latent heat, $C_{v}$ is volume heat capacity, and $\Delta T$ is the maximum temperature rise, respectively. The energy threshold $E_{t h}$ is determined by the interaction between the laser pulse and the target, which further includes many factors such as the laser wavelength, pulse duration, plasma losses and thermal properties of the target material. Since many of these parameters are material specific, the ablation process is different for different targets, or for different species in the same target, 
which explains the fact that the material flow in the plume is not uniform, especially at the outer edge of the cone-shaped plume where ions need to travel a longer path before reaching the substrate at a lower energy. Considering the transfer efficiency from the target to the films may vary for different elements, compensation on certain elements is occasionally made when mixing the source powders.

(2) Choice of substrates:

The fundamental rules that dictate the thin film epitaxy on a crystalline substrate are the lattice and thermal mismatches. For high quality, single-crystalline thin film growth, substrates that are in the same crystal system with similar symmetry as the desired thin film generally result in good epitaxy when the lattice mismatch is small. And hence, homoepitaxy, where the film and the substrate are the same material, results in films with highest possible quality.

In case of heteroepitaxy, where the film is a different material from the substrate, the difference in thermal expansion coefficients plays an important role. As the films are usually deposited at an elevated temperature, the shrinkage of the film will be different from that of the substrate during the post-growth cooling. If the thermal expansion coefficient of the film is larger than that of the substrate, the film shrinks more than the substrate during cooling and is bent concavely under tensile strain; on the contrary, if the film's thermal expansion coefficient is smaller and it shrinks less than the substrate, then the film is bent convexly with compressive strain. ${ }^{47}$ Exceedingly high tensile strain, especially in thick films, may crack the film after cooling. The residual strain in the film at room temperature can be estimated from ex situ asymmetric XRD measurements. ${ }^{48}$ As the residual strain slightly alters both short-range and long-range lattice arrangement, which in turn change the energy levels and band structure, physical properties of the film will also change as a result, e.g. a shift of the photoluminescence peak position in a $\mathrm{ZnO}$ film grown on $\mathrm{Si}^{43}$

The stacking of atoms in the film begins at the surface of the substrate, so the surface orientation of the substrate crystal also has a critical influence in the deposited film, which crystallizes in phases or orientations best matching the atomic structure of the substrate surface. 
Later in Chapter 3, we will see that single-crystalline (001) $\mathrm{Ca}$ : $\mathrm{TiO}_{2}-\mathrm{B}$ thin films can be grown on $\mathrm{SrTiO}_{3}$ substrates with a (001) surface, while the film becomes polycrystalline in different orientations on (110) $\mathrm{SrTiO}_{3}$ substrates. Such epitaxial relationship may be utilized to either optimize thin film growth, or to control and sometimes enhance certain properties of the film. An example will be presented in Chapter 5, where $\mathrm{Ca}: \mathrm{TiO}_{2}-\mathrm{B}$ thin films comprising rotated grains grown on a (110) $\mathrm{SrTiO}_{3}$ substrate deliver significantly enhanced battery performance.

In many property tests or device applications, thin films grown on particular substrate materials may be desired. For instance, measuring electrical properties by four probe Hall method requires a resistive substrate; measuring thermal conductivity by the $3 \omega$ method prefers that the substrate's thermal conductivity is much higher than that of the film; thin films with superior optoelectronic characteristics are often desired on Si substrates for easy integration with the current industrial infrastructure, etc. When the lattice and thermal mismatches between the films and the preferred substrate are too large for high quality growth, buffer layers are often used to mitigate the difference. As an example, single-crystalline $\mathrm{ZnO}$ films (hexagonal wurtzite) can be grown on $\mathrm{Si}$ (diamond cubic) with $\mathrm{Lu}_{2} \mathrm{O}_{3}{ }^{49}$ and $\mathrm{Sc}_{2} \mathrm{O}_{3}{ }^{48}$ buffer layers. Successful synthesis of a $\mathrm{SrTiO}_{3}$ buffer layer on $\mathrm{Si}$ also opens up numerous opportunities for depositing a series of perovskites on top. ${ }^{50}$

(3) Target-substrate distance:

The spatial distance between the target and the substrate is usually adjustable in modern PLD systems. Most PLD users place the substrate around the tip of the plume where it is fully extended. If the substrate is positioned too close to the target, the species in the plume will reach the substrate with very high kinetic energy, which may sputter off the film surface and increase its roughness. ${ }^{51}$ On the other hand, if the substrate is positioned too far from the target, the longer distance would allow greater segregation of different species in the plume that are travelling at different speeds, changing the stoichiometry when they arrive at the substrate, especially at the outer rim of the plume, and eventually causing variation in the thickness and chemical composition of the film. 
(4) Growth temperature:

The growth temperature refers to the temperature that the substrate is heated to and maintained at during deposition. It is a defining factor for the thermodynamic environment in which the elements condense into crystals at the substrate surface, and a critical parameter that determines the reaction rate. Different phases or different polymorphs of a material may form at different temperatures. If the target is heavily doped on a host material, or contains many species, phase segregation may occur at some temperatures, while forming a uniform compound phase at other temperatures. Hence, phase diagrams are often instructive in choosing a starting point for growth optimization, before trying out a range of temperatures. The dependence of the film quality on temperature is usually not exceedingly sensitive, so an interval of $50{ }^{\circ} \mathrm{C}$ often suffices when varying the growth temperature.

For some PLD systems, the substrate is in direct contact of the heating plate, to which the thermal couple is attached, so the reading on the controller is the actual temperature of the substrate. For other systems often equipped with a load-lock chamber for convenient substrate mounting, which are seen more and more recently, the substrate is separated from the heater by a gap of vacuum, and is heated by radiation. In the latter case, the temperature of the substrate must be calibrated because it is lower than that of the heater, and varies for different substrates due to the difference in emissivity of the material, which is associated with the thermal conductivity. The calibration can be performed by attaching a thermocouple to the substrate, or by using an optical pyrometer.

Within a certain temperature range in which the phase of the film does not change substantially, a higher temperature generally leads to higher structural quality. This is because the higher temperature facilitates a better mobility of the atoms along the film surface on which they continue to stack, resulting in a facile surface reconstruction and uniform distribution of the elements. In some cases, a higher temperature also helps with strain relaxation and grain growth, leading to larger grain sizes, fewer stacking faults, boundaries or other defects, and often times lower dislocation density. ${ }^{43,49}$ 
(5) Laser fluence:

The laser fluence is defined as:

$$
\text { Fluence }\left[\frac{\text { Joules }}{\mathrm{cm}^{2}}\right]=\frac{\text { Laser pulse energy }[\mathrm{J}]}{\text { Effective focal spot area }\left[\mathrm{cm}^{2}\right]}
$$

which should be distinguished from intensity and peak power, the two other terms often used with pulsed laser, defined as:

$$
\begin{gathered}
\text { Intensity }\left[\frac{\mathrm{Watts}}{\mathrm{cm}^{2}}\right]=\frac{\text { Laser peak power }[\mathrm{W}]}{\text { Effective focal spot area }\left[\mathrm{cm}^{2}\right]} \\
\text { Peak power }[\mathrm{W}]=\frac{\text { Laser pulse energy }[\mathrm{J}]}{\text { Pulse duration }[\mathrm{s}]}
\end{gathered}
$$

Since most PLD systems use similar laser models, such as the COMPex 205 for the work in this thesis, which have a pulse duration in the scale of nanoseconds (ns), fluence is the most commonly used term to describe the laser pulse energy in the literature.

The laser fluence determines the amount of material that is ablated from the target surface, and the kinetic energy the species carry. Experimentally, higher laser fluence excites a stronger and larger plume, and usually increases the film deposition rate until a saturation point governed by the crystallization thermodynamics is reached. The bigger plume coverage is also favorable for a uniform film growth on a large substrate. Similarly to a small target-substrate distance, exceedingly high laser fluence may cause an increase in the film roughness. Furthermore, an over-supply of metallic ions may sometimes force the film to crystallize in a different phase. For instance, as will be presented in Chapter 3, $\mathrm{TiO}$ phase could form instead of $\mathrm{TiO}_{2}$ when the laser energy is set too high ablating a target containing $\mathrm{TiO}_{2}$. On the other hand, lower energy fluence often results in smoother films with better structural quality, as long as the threshold of species energy and respective ion concentrations are met for the desired phase to form. For targets that are dense or containing heavy elements such as $\mathrm{Pt}$, the laser fluence required to generate a strong enough plume is higher.

Ultrafast PLD which employ low energy pulses in the picosecond (ps) or femtosecond (fs) regimes has also been investigated. ${ }^{51}$ The major merit of such technique is that the pulse width is 
shorter than the electron-phonon time scale in solids (typically a few ps), and therefore thermal effects in the target can be neglected. However, the high pulse power density $\left(\sim 10^{15} \mathrm{~W} / \mathrm{cm}^{2}\right)$ resulting from the brevity of the pulse induces high kinetic energy $(\sim 1 \mathrm{keV})$ species in the plume, which likely leads to a large structural disorder and surface roughness in the films.

(6) Laser repetition rate:

The repetition rate is the number of pulses emitted per second from the laser, expressed with the unit of $\mathrm{Hz}$. Increasing the repetition rate directly increases the amount of material ablated from the target in any given time, and is usually an effective method to enhance the deposition rate of the thin films, which is conventionally described by $\AA /$ pulse, provided that the saturation growth rate is not yet reached. Similarly to using high laser pulse energy, a high repetition rate may also cause lower structural quality, as the lattice has less time for lateral reconstruction and grain growth before its vertical stacking. In practice, PLD users often search for a compromise between the deposition rate and the structural quality, and a repetition rate of $1-10 \mathrm{~Hz}$ is mostly applied.

(7) Gas backfill pressure:

The gas backfill pressure controls the mean-free path of species composing the plume, their respective kinetic energies and the plume angular distribution. For ceramic thin film growth, such as many semiconductors or other functional metal-oxides, oxygen is most commonly used backfill gas. This is because most ceramic targets are oxygen deficient from the sintering process, and more oxygen is further lost during ablation since it is much lighter than the metal ions and easily carried away by the vacuum pump suction. An oxygen ambient therefore counteracts the deficiency and enhances structural quality of the films.

Using a higher oxygen partial pressure, other than causing a weaker plume, is essentially creating a more oxidizing environment for the films to grow. For instance, in a research work included in Appendix B, we have found that from the same Pt doped $\mathrm{SrTiO}_{3}$ target, uniform $\mathrm{SrTi}_{1-\mathrm{x}} \mathrm{Pt}_{\mathrm{x}} \mathrm{O}_{3}$ films were grown under high oxygen pressure, while Pt particle precipitation out of the host matrix was observed in films grown under low oxygen pressure. First principles 
calculations may sometimes suggest the level of redox conditions needed for a desired phase to form, which thus provides valuable guidance for experimental investigations.

Another major effect of the oxygen partial pressure is reflected by the number of oxygen vacancies in the thin films. Higher pressure generally leads to fewer oxygen vacancies. For a semiconductor thin film, an oxygen vacancy is an $n$-type carrier, so changing the oxygen pressure during growth is actually an effective method to control the electrical properties of the film. Apparently, if a p-type film is desired, higher oxygen pressure should be used to avoid charge carrier counteraction, while in an $n$-type film, lower oxygen pressure may help increase the carrier concentration and electrical conductivity. If a high concentration of oxygen vacancies is preferred provided that the crystal structure still remains stable, an inert gas ambient, such as Ar, may be used. ${ }^{52}$

After the deposition is finished, the same oxygen partial pressure may be maintained during cooling, or the gas supply can be turned off for a vacuum cooling process. Since the post-growth cooling is essentially half of an annealing cycle, the choice of whether to keep the gas flow should be made according to the purpose. To obtain a film with better structural quality, oxygen backfill is often kept to fill up the vacancies. Vacuum cooling followed by another thermal treatment may yield different microstructures, while also provides an intermediate stage for property testing.

Besides the chamber pressure as a result of gas backfilling, the directions of the gas flow may sometimes have an effect on the deposited film, ${ }^{53}$ since the flow can change the shape of the plume, or affect the paths of various species. In the PLD system that produced most of the samples for this thesis, the gas inlet and the port to the turbopump are positioned such that the gas flow is skimming the inner wall of the chamber, while the vacuum gauge is placed near the substrate and out of the gas flow. Hence the partial pressure reported in this thesis is a more accurate reflection of the chamber pressure, and the direction of the gas flow is ignored. 


\subsubsection{The PLD systems}

The $\mathrm{TiO}_{2}-\mathrm{B}$ and $\mathrm{Ca}: \mathrm{TiO}_{2}-\mathrm{B}$ thin films in this study were grown in two different PLD systems that shared a $\mathrm{KrF}$ excimer laser. One system was customized with major components purchased from Neocera LLC, which produced most of the films discussed in this thesis. The rest of the bronze films, as well as the Pt-Nb co-doped $\mathrm{SrTiO}_{3}$ films investigated in Appendix B, were fabricated in another system manufactured by Thermionics Laboratory Inc. Photographs of the laser, plasma plume and the two PLD systems can be found in Ref. 59, and are provided here in Figure 2.2.

The KrF excimer laser (Lambda-Physik COMPex 205) emits laser pulses with a wavelength of $248 \mathrm{~nm}$ and a pulse duration of $22 \mathrm{~ns}$. The beam cross section is $8 \times 22 \mathrm{~mm}^{2}$. The laser tube is water-cooled and uses refillable premix gas of krypton-helium-fluorine-neon (3.82\%-1.68\%-0.09\%-94.41\%). In this study the laser repetition rate was set to $10 \mathrm{~Hz}$ and the beam energy was in the range of $200-400 \mathrm{~mJ}$ for most films. The laser beam is focused by a fused silica lens onto the target with a spot size of approximately $1 \times 2 \mathrm{~mm}^{2}$. Considering the pulse energy loss in the optics, the effective fluence was $\sim 3.4-6.8 \mathrm{~J} \mathrm{~cm}^{-2}$.

The Neocera PLD system consists of a 14.5" diameter stainless steel vacuum chamber equipped with a conductive heating substrate stage, a multi-target carrousel, a Varian SH-100 dry scroll mechanical pump, and a Varian V-301 turbopump. The 2" flat-top heating stage can heat up to $900-950{ }^{\circ} \mathrm{C}$, on which the substrates are held using silver paste as adhesive, so the growth temperature control for the substrates is quite accurate with this configuration. The multi-target carrousel holds six targets at one time, where two servo motors control the rotation and rastering of the targets, respectively. The mechanical fore-pump is used for pre-stage pumping of the chamber down to $\sim 50$ mTorr. The turbopump further lowers the chamber base pressure to $<10^{-4}$ mTorr before deposition. The distance between the target and the substrate is fixed at $6.35 \mathrm{~cm}$ in this system. During deposition, backfill gases such as $\mathrm{O}_{2}, \mathrm{~N}_{2}$ and $\mathrm{Ar}$ are introduced through a needle leakage valve. The gas background pressure is adjusted between vacuum and 100 mTorr. A major advantage of this system is that the substrates are glued to the heating stage, so 
substrates of any size and shape can be used.

The Thermionics PLD system is an ultra-high vacuum system, which has a stainless steel 22" diameter cylindrical main chamber evacuated by a Varian V-551 turbopump backed by a Varian TriScroll 300 dry-pump. The chamber base pressure is $\sim 10^{-7}$ mTorr. A load-lock chamber (LLC), sealed by a gate valve, is installed to transfer substrates and targets in and out using a magnetic mechanical arm, without having to break the vacuum in the main chamber, thus greatly enhancing the workflow efficiency. The LLC is equipped with a Pfeiffer TMU-071P air-cooled turbopump backed by a Pfeiffer MVP 035-2 diaphragm pump. The substrate manipulator is equipped with an $\mathrm{XYZ}$ stage. The $\mathrm{XY}$ micrometers allow $\mathrm{a} \pm 0.5^{\prime \prime}$ travel and the Acme drive allows a $\mathrm{Z}$ travel of 4 " into and out of the chamber. In addition, the substrate manipulator can continuously rotate for $360^{\circ}$, where it is vacuum-sealed by a two-stage differentially pumped rotary seal (RNN) connected to an ion pump. This allows fine positioning of the substrates in the vacuum chamber, facing the target at a distance adjustable between 1.5" and 2.5". Substrates are radiatively heated by a $\mathrm{Pt}_{0.8} / \mathrm{Rh}_{0.2}$ filament integrated in the manipulator. A type-K thermocouple is placed closely to the filament to monitor the heater temperature. The maximum filament temperature is $1100^{\circ} \mathrm{C}$, while the actual substrate temperature needs to be calibrated by placing a second thermocouple directly in contact with the substrate (see Section 2.1.2: Growth temperature). The substrate manipulator is also equipped with a motorized feedthrough for azimuthal rotation of the substrate during growth, in order to achieve uniform film structure and thickness. Hollow substrate holders specially designed for radiative heating accept substrates with sizes of $10 \times 10 \mathrm{~mm}^{2}, 5 \times 5 \mathrm{~mm}^{2}$, and 2" diameter. The target manipulator can hold six targets vertically in-line at one time, and is operated on a dual-axis rotary feedthrough. One axis is used for vertical clocking of the targets and the other is for spinning. The vacuum level in the main deposition chamber is monitored by a Pfeiffer full range Pirani inverted magnetron gauge able to measure from $5 \times 10^{-9}$ to 1000 mbar. One major merit of the Thermionic system is that the substrates are held by Ni clips without any adhesives, so the cross-contamination inside the chamber is considerably lower than that in the Neocera system (silver paste). 


\subsubsection{Target and substrate preparation}

In addition to the general considerations for targets and substrates discussed previously in Section 2.1.2, more details in the experimental preparation are provided here.

A smooth surface and a high density are the generally desirable features of a target in order to minimize splashing (ejection of molten liquid from the ablation front causing droplets on the film surface) ${ }^{46}$ The ceramic targets used for PLD growth in this thesis were all home-made by mixing and thoroughly ball-milling source powders at the designed ratio, which were then uniaxially cold pressed into a pellet of 1 " diameter under $10000-15000 \mathrm{lb}$ of force $(2.205 \mathrm{lb}=1$ $\mathrm{kg}$ ) and sintered at $1000-1400{ }^{\circ} \mathrm{C}$. The sintering process is for the ingredients in the green body to fully mix and react, forming a dense PLD target. The ramp rate was $1{ }^{\circ} \mathrm{C} / \mathrm{min}$, for heating and cooling, while the dwelling time at the sintering temperature was usually 48-72 hours. In an alternative recipe, the cooling process was broken into three parts, where the first part was controlled cooling at the set ramp rate $\left(1^{\circ} \mathrm{C} / \mathrm{min}\right)$, then the pellet was held at an intermediate temperature (e.g. $\left.600^{\circ} \mathrm{C}\right)$ for 1 hour, and finally a natural cooling to room temperature. In certain cases this recipe yielded a more uniform, better shaped and crack-free target with flat surfaces as the added steps helped with the shrinkage and strain relaxation during cooling in the bulk. Although not employed in this study, using a hot press instead of a cold press may further improve the target uniformity. Prior to each growth, the target was pre-ablated for a few minutes to remove possible surface contaminants and expose the fresh material. After being used for a while, the targets were periodically taken out of the chamber and re-polished with SiC grit paper for a smooth surface.

The single crystal substrates used in this study were purchased from MTI Corporation. These commercial substrates, other than $\mathrm{Si}$, were single-side polished to epi-ready smoothness, and precision-cut to $10 \times 10 \mathrm{~mm}^{2}$ with a $0.5 \mathrm{~mm}$ thickness. The single-side polished Si substrates (intrinsic and $p$ - or $n$-doped) were originally $3 "$ wafers (also $0.5 \mathrm{~mm}$ thick) and diced into $10 \times 10$ $\mathrm{mm}^{2}$ squares using an ADT-7100 dicing saw. Before dicing, the wafers were spin-coated with a 
thin layer of photoresist (SPR 220) using a CEE 100CB spinner for protection. After dicing, the photoresist was completely removed by acetone. Before being loaded into the PLD chamber, the substrates were ultrasonically cleaned in acetone, rinsed in sequence with acetone, methanol and de-ionized (DI) water, and finally blown dry with $\mathrm{N}_{2}$ gas. It should be noted that $\mathrm{Si}$ substrates are especially brittle, and tiny Si splinters may come off of the cutting edges upon vibration and stick to the surface, which are very difficult to remove. Therefore the ultrasonic cleaning time should be brief, usually 10-30 seconds, for Si substrates, while a few minutes are good for hard substrates such as sapphire and perovskites $\left(\right.$ e.g. $\left.\mathrm{SrTiO}_{3}\right)$. Prior to deposition, the substrates were heated up in vacuum to avoid surface oxidation, and oxygen ambient gas was backfilled once the set growth temperature was reached and maintained at the same pressure throughout the deposition and cooling process.

$\mathrm{SrTiO}_{3}$ buffered $\mathrm{Si}$ substrates were also used, provided by Prof. Darrell Schlom at Cornell University. The buffer layers contained 20 or 40 unit cells of $\mathrm{SrTiO}_{3}$ crystal, grown on (100) Si by reactive molecular-beam epitaxy $(\mathrm{MBE}){ }^{50}$

Crystal information of the substrates used for thin film deposition will be listed later in this thesis, where the growth details of particular films are discussed.

\subsubsection{PLD growth of the Ca: $\mathrm{TiO}_{2}-\mathrm{B}$ and $\mathrm{TiO}_{2}-\mathrm{B}$ thin films}

Thin film growth optimization is a systematic process aimed at finding the optimal combination of the parameters, often guided by structural characterization and property tests along the way. Chapter 3 depicts such a process in great detail, from first observing the $\mathrm{Ca}: \mathrm{TiO}_{2}-\mathrm{B}$ and $\mathrm{TiO}_{2}-\mathrm{B}$ phases as defects in $\mathrm{CaTiO}_{3}$ films to single crystal fabrication with high phase purity. Here a brief preview is offered into that process, and how it is connected to the growth conditions discussed above in Section 2.1.2.

It was not clear at the early stage what the $\mathrm{Ca}-\mathrm{Ti}$ ratio is in the $\mathrm{Ca}: \mathrm{TiO}_{2}-\mathrm{B}$ phase, so a number of targets with different $\mathrm{Ca}$ content were made, starting from $10 \% \mathrm{Ca}$. The growth conditions for $\mathrm{CaTiO}_{3}$ films previously were used as the starting point. A few batches were then 
grown, with 3-4 different substrates installed in the chamber for every batch, at various temperatures in $100{ }^{\circ} \mathrm{C}$ steps and under different $\mathrm{O}_{2}$ partial pressure, centering the initial parameters. Each film was examined for phase composition and crystal quality using the techniques described in Section 2.2. The $\mathrm{Ca}: \mathrm{TiO}_{2}-\mathrm{B}$ phase was observed in a few samples as small grains, which concluded the first stage when a rough estimation was achieved on the possible conditions for the new phase to form.

The second stage was aiming at expanding the $\mathrm{Ca}: \mathrm{TiO}_{2}-\mathrm{B}$ grain size until a uniform film was obtained. Increasing growth temperature helped the grain growth significantly. Increasing the flux of ions from the target to the substrate was another important measure, which was executed by raising the laser energy and repetition rate, until undesirable phases started to form or the crystal quality began to deteriorate. After a good combination of these conditions is found, a longer deposition time was used to produce thicker films. The end result of this stage was a uniform $\mathrm{Ca}: \mathrm{TiO}_{2}-\mathrm{B}$ film but containing considerable amount of the $\mathrm{TiO}_{2}$-anatase phase $(>10 \%)$.

The third stage was to optimize the bronze phase purity and to further improve the crystal quality. By this point, the deposition parameters were narrowed down to small ranges, and the structure of the $\mathrm{Ca}: \mathrm{TiO}_{2}-\mathrm{B}$ phase was clear from theoretical calculations. The optimization at this stage requires fine tuning of the conditions in small steps, e.g. changing the temperature in $50{ }^{\circ} \mathrm{C}$ steps or even smaller. The microstructures of every sample should be studied carefully. While simple appearance of the $\mathrm{Ca}: \mathrm{TiO}_{2}-\mathrm{B}$ peaks in diffraction patterns would suffice earlier, now the strength and width of both the $\mathrm{Ca}: \mathrm{TiO}_{2}-\mathrm{B}$ and anatase peaks need to be tracked, and the causes should be clearly understood, for which TEM/STEM images are especially helpful. Reasonably higher $\mathrm{O}_{2}$ pressure may help reduce oxygen vacancies in the oxide matrix and thus reduce crystal defects, without favoring other phases that prefer highly oxidizing condition; slightly lower laser repetition rate may slow down the deposition rate but in return improve the film quality by reducing stacking faults. The most effective method to suppress the anatase phase formation was found to be an over-compensation of $\mathrm{Ca}$ above the stoichiometry content, and single-crystalline $\mathrm{Ca}: \mathrm{TiO}_{2}-\mathrm{B}$ films were obtained by using a target containing $20 \% \mathrm{Ca}$. 
Each of the three stages described above took approximately $1 / 3$ of the time in the optimization process. This is expected to be roughly true for other material systems as well.

Once the high quality $\mathrm{Ca}: \mathrm{TiO}_{2}-\mathrm{B}$ growth was achieved, it was not long before we found that regular $\mathrm{TiO}_{2}-\mathrm{B}$ single-crystalline films could be grown on a $\mathrm{Ca}: \mathrm{TiO}_{2}-\mathrm{B}$ template layer,

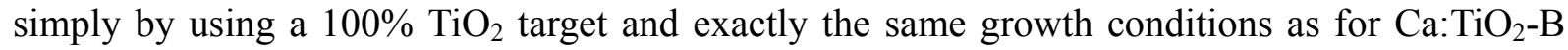
(see Section 3.5).

Now that the conditions are well refined, the fourth and final stage for synthesizing the bronze films was to grow them for various practical purposes. The best film quality was achieved on (001) $\mathrm{SrTiO}_{3}$ substrates, followed by other similar cubic crystals including $\mathrm{LaAlO}_{3}$ and LSAT (see details in Chapter 3). Nb-doped $\mathrm{SrTiO}_{3}$ substrates were used to grow films for electrochemical testing, where a conductive current collector was needed (see Section 4.2.1). Polycrystalline $\mathrm{Ca}: \mathrm{TiO}_{2}-\mathrm{B}$ thin films with enhanced high rate battery performance were obtained on (110) $\mathrm{SrTiO}_{3}$ (see Chapter 5).

\subsection{Structural characterization}

Structural characterization of the thin films was carried out by x-ray diffraction (XRD), atomic force microscopy (AFM), and transmission electron microscopy (TEM).

\subsubsection{Film thickness measurement}

The bronze film thickness was measured by a Veeco Dektak profilometer, which scanned a fine tip along a straight line from an area on the sample where the bare substrate was exposed to an area covered with the film, and read the step height. For films deposited in the Thermionics system, clips on the substrate holder acted as masks during growth and left small areas on the substrate exposed. For films deposited in the Neocera system, the entire substrate was covered with the film, so a small piece of the film needed to be gently scraped off the substrate for the measurement. Typical films in this study have thicknesses of 50-200 nm.

Many of the films have been studied by TEM, and the images further confirmed the 
thicknesses measured by the profilometer. If a discrepancy occurred between the two measurements, the value acquired by TEM was used for further analysis, such as in calculating the mass of the film and the specific capacity for battery testing.

\subsubsection{X-ray diffraction}

All Ca: $\mathrm{TiO}_{2}-\mathrm{B}$ and $\mathrm{TiO}_{2}-\mathrm{B}$ films were characterized by two x-ray diffractometers in the $\mathrm{J}$. D. Hanawalt X-ray MicroAnalysis Laboratory. The first is a Rigaku Rotaflex rotating anode diffractometer operated at $40 \mathrm{kV}$ and $100 \mathrm{~mA}$, designed mainly for $\theta-2 \theta$ scans and pole figures. The second is a BEDE D1 triple-axis high resolution diffractometer operated at $40 \mathrm{kV}$ and 40 $\mathrm{mA}$, which offers precision movement of the sample stage in all six degrees of freedom, especially powerful in off-axis XRD experiments such as for $\omega$-scans (rocking curves) and $\phi$-scans. Both instruments use monochromatic $\mathrm{Cu}$ - $\mathrm{K} \alpha$ radiative sources $(\lambda=1.54 \AA)$.

\subsubsection{1 $\theta-2 \theta$ scan}

The $\theta-2 \theta$ scans were performed in the Rigaku diffractometer for the bronze thin films. A $0.5 \mathrm{~mm}$ source slit was used to limit the width of the x-ray beam. During the scan, the sample was placed vertically and rotated by an angle of $\theta$, while the detector was simultaneously rotated by an angle of $2 \theta$, with respect to the horizontal incident $\mathrm{x}$-ray beam. The x-ray source was not moving. The scanned $2 \theta$ range was set to $5^{\circ}-130^{\circ}$. The scans were set to the continuous mode at a speed of $3 \% / \mathrm{min}$ and the $2 \theta$ increment was $0.01 \%$ step. The thin film samples with a surface area $\leq 1 " \times 1$ " were fixed by clay on the sample holder.

Unlike experiments on powder samples, where the particles are randomly oriented and the XRD pattern represent a collective effect, for epitaxial films, the crystal orientation is fixed and only lattice planes parallel to the film surface will appear in a $\theta-2 \theta$ scan where the Bragg's law is satisfied: 


$$
n \lambda=2 d \sin \theta
$$

where $n$ is an integer, $\lambda$ is the $\mathrm{x}$-ray wavelength, and $d$ is the spacing between the lattice planes. Therefore, prior to the complete scan, the sample surface was first aligned by a pre-scan of the substrate surface reflection (e.g. $\mathrm{SrTiO}_{3} 100$ reflection). For high quality single crystal substrates, the peak intensity was up to $\sim 5 \times 10^{4}$ counts on the detector. The XRD pattern of a highly crystalline film usually reveals a group of strong peaks corresponding to a series of planes parallel to the surface, the intensities of which are not proportional to those in the standard powder diffraction files (PDFs).

It is worth noting that due to such limitation, the $\theta-2 \theta$ scan results cannot provide definite and comprehensive insight into the phase composition of the film, since lattice planes, sometimes in a tilted grain, which are not parallel to the surface will not show in the $\theta-2 \theta$ patterns. The investigation of the film structure is thus best aided by the TEM method, or by pole figures that are more efficient but less accurate and intuitive.

\subsubsection{2 $\omega$-scan}

The $\omega$-scan of the epilayers, sometime referred to as $\omega$-rocking curves, was performed in the BEDE D1 diffractometer. A high resolution scan was carried out by limiting the x-ray beam width with a channel cut collimator (CCC) crystal and two source slits $(1 \mathrm{~cm}$ diameter and 0.5 $\mathrm{mm}$ width, respectively). During the scan, the detector was fixed at the $2 \theta_{\mathrm{B}}$ Bragg angle of the reflection under study (e.g. $\left.006 \mathrm{Ca}: \mathrm{TiO}_{2}-\mathrm{B}\right)$ and the vertically placed sample was 'rocked' within $2-3^{\circ}$ deviation from $\theta_{\mathrm{B}}$. The scan speed was set between $0.1-0.5 \% \mathrm{~min}$ and the scan increment was $0.005 \%$ step. The sample was fixed by double-sided tape on the sample holder with its surface parallel to the incident $\mathrm{x}$-ray beam, and the position was pre-aligned so the thickness of the sample cuts half the width of the x-ray beam by adjusting the triple axes (XYZ) and optimizing the tilt angle ( $\chi$-scan) of the sample holder. The diffractometer axes and angles are referred to as in Figure 2.3.

The broadening of the rocking curve, i.e. its full width at half maximum ( $\omega$-FWHM), 
which represents the mosaic misorientation of the XRD reflection, is an important (and usually convenient) marker to evaluate the crystalline quality of a epitaxial thin film. For lithium hosts in electrochemical studies, where the Li ions changes the lattice structure upon insertion, this parameter can also be used to reveal the difference in structural quality before and after lithiation. The $\mathrm{Ca}: \mathrm{TiO}_{2}-\mathrm{B}$ and $\mathrm{TiO}_{2}-\mathrm{B}$ epilayers discussed in this thesis have strong symmetric, out-of-plane 006 and 001 reflections, respectively, the broadening of which have been measured to compare the crystal quality between films grown under different conditions. Though not used in this study, such technique may further be used to estimate the degree of the in-plane misorientation, where a reflection from planes with higher indices, which are tilted at an angle with the film surface, is measured in skew symmetric geometry, where the sample holder is first inclined by a tilt angle $(\chi)$ equaling to the intersection angle between the surface plane and the measured plane, and then rotated by an azimuthal angle $(\phi)$ about the normal of the measured plane to satisfy the Bragg condition. More details on the skew symmetric $\omega$-scan can be found in Ref. 59.

\subsubsection{Pole figure}

The right arm of the Rigaku diffractometer is capable of pole figure measurements, which are useful to determine the texture of the thin films, as well as the in-plane orientation relationship between the film and the substrate. It is efficient to perform a pole figure measurement that automatically sweeps over a range of tilt angles, especially when the film structure is largely unknown, in order to identify new phases and locate lattice planes which are not parallel to the surface and thus not discoverable in $\theta-2 \theta$ scans. The sample was fixed by clay on a round triple-axis rotating plate.

To set up a pole figure measurement, the sample plate was positioned to the Bragg angle $\left(\omega=\theta_{\mathrm{B}}\right)$ of the desired plane, and the detector was fixed at $2 \theta_{\mathrm{B}}$. For the bronze films studied in this thesis, the $2 \theta_{\mathrm{B}}$ angle was set at $30.22^{\circ}$ for $\mathrm{Ca}: \mathrm{TiO}_{2}-\mathrm{B} 006$ peak, and after the scan starts, the sample plate was inclined from $\chi=0^{\circ}$ to $\chi=75^{\circ}$ with a step of $2.5^{\circ}$. A $360^{\circ} \phi$-scan, where the sample plate rotates about its surface normal with a step of $5^{\circ}$, was measured at each $\chi$ angle. An 
XRD pole figure is drawn by plotting all these $\phi$-scans plotted on the same polar diagram. Conventionally, the label on the polar axis refers to $90^{\circ}-\chi$. It is very difficult to align the sample surface perfectly in position, so the intensities of the poles are usually not homogeneous.

\subsubsection{Atomic force microscopy}

The film surface structure was characterized by a Veeco NanoMan AFM located in the Lurie Nanofabrication Facility (LNF). The microscope was operated under tapping mode, imaging the surface morphology by tracking a small silicon cantilever over the film surface and recording the oscillation of the coupled laser signals. The cantilever was pre-tuned before imaging, oscillating near the resonance frequency around $300 \mathrm{kHz}$. The typical scan area for the $\mathrm{TiO}_{2}-\mathrm{B} / \mathrm{Ca}: \mathrm{TiO}_{2}-\mathrm{B}$ films was $5 \times 5 \mu \mathrm{m}^{2}$, and the images were recorded at a scan rate of $1-2 \mu \mathrm{m} / \mathrm{s}$. The scan resolution can be improved by optimizing the scan rate, the proportional gain, and the integral gain of the cantilever. The vertical offset induced by artifacts such as sample tilting and drifting was removed by a flattening function using the NanoScope Analysis software, which also provided the root-mean-square (rms) roughness and 3D image processing.

\subsubsection{Transmission electron microscopy}

Microstructures and defects in the bronze films were studied at the atomic scale by scanning transmission electron microscopy (STEM) using a JEOL 2100F TEM equipped with a spherical aberration corrector, located at the Electron Microbeam Analysis Laboratory (EMAL). TEM specimen preparation and imaging were performed by our research group members Michael Katz and Sung Joo Kim.

The JEOL 2100F STEM is an advanced field emission electron microscope equipped with a spherical aberration (Cs) hexapole STEM probe corrector (CEOS GmbH). The electron beam is accelerated by a voltage of $200 \mathrm{kV}$. The TEM point-to-point resolution is $0.19 \mathrm{~nm}$. The STEM probe size is $<0.1 \mathrm{~nm}$. Z-contrast image is obtained in the STEM mode by focusing and rastering the electron probe across the specimen while using a Fischione high angle angular dark 
field (HAADF) detector to collect the scattered electrons. The brightness of atoms in the $Z$-contrast images is approximately proportional to the square of the atomic numbers $(Z)$.

Preparation of the TEM specimen consists of standard processes of mechanical grinding, polishing, precision dimpling, and ion milling, with the end result of an electron transparent edge which is then examined in the microscope. High quality TEM imaging also requires little artificial damage to the specimen during sample preparation. Detailed information of the preparation procedure can be found in Ref. 54. The precise cutting direction, if not immediately available from the substrate itself, can be determined by finding the correct azimuthal angle in skew symmetric XRD $\omega$-scan of off-axis reflections. ${ }^{59}$

In addition to imaging, TEM/STEM can be used with various spectroscopic techniques in order to characterize the physical and chemical nature of the specimen, including $\mathrm{x}$-ray energy dispersive spectroscopy (XEDS) and electron energy loss spectroscopy (EELS), each offering a way to obtain information on the elemental composition.

XEDS, sometimes referred to as EDS or EDX, is more commonly used between the two. In an XEDS experiment, an atom may absorb from impinging electron beam an energy high enough to eject an electron from an inner shell, creating a hole in the core level. An electron from an outer, higher-energy shell then fall into the hole, and the difference in energy between the two shells is released in the form of an x-ray, which is characteristic to the particular transition, and thus to a particular element. The emitted x-ray is collected by a detector and analyzed by computer software to produce a spectrum with elemental identification across most of the periodic table, while the technique is generally less efficient for elements lighter than carbon. The major disadvantage of XEDS is its low collection efficiency, because the detector can only cover a small solid angle in the $3 \mathrm{D}$ space $(\sim 0.05-0.1$ steradian) while the $\mathrm{x}$-ray from the specimen travels in all directions. Experimentally, a compromise needs to be made between moving the detector closer to the specimen and keeping enough distance so it is not shadowed by the sample holder.

EELS has played an important role in identifying the structure of the new $\mathrm{Ca}: \mathrm{TiO}_{2}-\mathrm{B}$ 
phase in this work. The EELS signal results from an impinging electron interacting inelastically with atoms in the specimen, partially losing its energy while continuing down the TEM column. The electron taking the energy is either ejected out of the atom, or excited into an unoccupied, higher energy orbital. Therefore, the energy that the beam electron loses has a definite value which is unique to the specific element, resulting in a characteristic signal, or EELS edge, in the spectra. After passing through the specimen, the electron beam now consists of electrons with different energies, which are dispersed by a magnetic prism, where lower energy electrons have more curved paths through the prism. Eventually the dispersed electrons are focused onto a CCD (charge-coupled device) camera acting as the EELS detector. The EELS method is especially powerful when the specimen is thin. Since almost all of the inelastically scattered electrons continue down the column and get collected in the entrance aperture of the EELS detector, the signal-to-noise ratio is very high. However, not all elements produce clear EELS edges. For instance, Pd produces a very broad edge that is often damped out.

When used in conjunction with STEM, which is able to place a sub-Ångstrom probe at any point of the specimen, highly localized XEDS and EELS measurements with atomic resolution can be realized. For samples discussed in this thesis, XEDS and EELS were performed in the TEM instrument described above with XEDS detectors from JEOL and EDAX, and Gatan Image Filter EELS system, respectively.

\subsection{Electrical characterization}

The electrical properties of the bronze films were characterized by Hall effect measurements in the Van der Pauw configuration. ${ }^{55}$ The National Institute of Standards and Technology suggests the preferred, acceptable, and not recommended sample geometries for Hall measurements, as shown in Figure 2.4. ${ }^{56}$ Experimental error increases with increasing size of the contacts. The configuration used in this study is Figure 2.4 (b), where the contacts were smaller than $0.5 \times 0.5 \mathrm{~mm}^{2}$ in size. For typical $5 \times 5 \mathrm{~mm}^{2}$ and $10 \times 10 \mathrm{~mm}^{2}$ samples, such contact size induces about $5-10 \%$ relative error. The contacts were made by pressing indium dots on the 
corners of the sample, bonding with thin gold wires, and annealing at $200{ }^{\circ} \mathrm{C}$ in $\mathrm{N}_{2}$ gas flow for 1 min. For semiconductor thin films with sufficient electrical conductivity, linear dependence of current on varying voltage (i.e. I-V curve) between any two contacts should be obtained prior to each measurement, which is usually referred to as an Ohmic contact. Unless the film has an exceedingly high electrical conductivity in the regime of a semimetal, the contact resistance is often neglected.

Other geometries proposed to improve the accuracy of the Hall effect measurement are presented in the book from Putley et al. ${ }^{57}$

Hall measurements on the bronze films in this thesis were performed on two different systems. The first one is an MMRH-50 Hall Measurement System equipped with a $0.64 \mathrm{~T}$ permanent magnet, graciously offered for use at IMRA America, Inc. The second one is a customized system integrated with the cryostat of the photoluminescence (PL) characterization system, equipped with a $0.22 \mathrm{~T}$ permanent magnet, a Keithley 220 current source, and a Keithley 6517A high resistance meter. The closed-cycle cryostat (ARS, Displex, CS-202) is cooled by a He gas compressor, and is operated with accurate temperature control between $10 \mathrm{~K}$ and $320 \mathrm{~K}$. The sample is mounted on the cold finger by conductive copper tape for good heat conduction. If the thin film was grown on an conductive substrate, non-conductive double-sided tape is used instead, and a longer idle period is adopted every time the temperature setting is changed, allowing the sample temperature to reach equilibrium with that of the cold finger. The cryostat is first evacuated to a pressure of $10^{-5}$ mbar by a Pfeiffer TSH-071E turbopump station, and the He compressor is turned on after $15 \mathrm{~min}$, which cools the cryostat to $10 \mathrm{~K}$ in about $90 \mathrm{~min}$. A laser beam from a He-Cd laser (Kimmon, IK3501R-G, $\lambda=325 \mathrm{~nm}, 100 \mathrm{~mW}$ ) is focused on the film surface, which excites luminescence in a PL measurement. If used in the Hall measurement, the incident laser may also excite more conducting carriers in the sample, changing its electrical properties. For example, if the film is an $n$-type material with a band gap $<3.8 \mathrm{eV}$, the laser energy can be absorbed to elevate more electrons into the conduction band, significantly increasing the carrier concentration and electrical conductivity. 
The resistivity $(\rho)$ of the sample is determined without a magnetic field $(B=0)$ by measuring the voltages $\left(V_{i j}\right)$ across two adjacent contacts while running currents $\left(I_{k l}\right)$ through the other two contacts. The measurements are repeated eight times by rotating among the four contacts and reversing the direction of current each time. The resistivity is thus given by: ${ }^{55}$

$$
\rho=R_{S} d=\frac{\pi d}{\ln 2} \frac{\overline{V_{l j}}}{I_{k l}} f \quad(i, j, k, l=1,2,3,4)
$$

where $R_{S}$ is the sheet resistance, $d$ is the film thickness, and $f$ is the sample geometry factor which takes values between 0 and 1 . For a symmetric sample, $f=1$. More details for determining the $f$ value can be found in Ref. 55. The average of eight $V_{i j} / I_{k l}$ values eliminates potential errors caused by non-uniformity of the thin film, misalignment of the contacts, and other possible anisotropic effects in the material.

In Hall voltage measurements, current runs diagonally through the sample (e.g. $\left.I_{13}\right)$ and voltage is measured across the other diagonal twice for $+B$ and $-B$ magnetic fields, respectively (e.g. $V_{24}^{+B}, V_{24}^{-B}$ ). The measurements are repeated four times by rotating the contacts and reversing the current direction. The conduction type of the sample, carrier concentration $(n)$ and Hall mobility $(\mu)$ are calculated by: $:^{56}$

$$
\begin{gathered}
\left\{\begin{array}{l}
p-\text { type, } \quad \text { if } \sum\left(V_{i j}^{+B}-V_{i j}^{-B}\right)>0 \\
n-\text { type, } \quad \text { if } \sum\left(V_{i j}^{+B}-V_{i j}^{-B}\right)<0
\end{array}, \quad i j=13,31,24,42\right. \\
n=\frac{n_{S}}{d}=\frac{8 \times 10^{-8} I B}{e d} \frac{1}{\sum\left(V_{i j}^{+B}-V_{i j}^{-B}\right)} \\
\mu=\frac{1}{e \rho n}
\end{gathered}
$$

where $n_{s}$ is the sheet carrier density and $e$ is the elementary charge.

\subsection{Electrochemical property testing}

\subsubsection{Testing techniques}

Electrochemistry deals with the interaction between electrical energy and chemical change, which studies the chemical reactions that take place at the interface of an electrode, 
usually a solid metal or a semiconductor, and an ionic conductor, the electrolyte. These reactions involve electrical charges carried by ionic species moving between the electrodes and the electrolyte. In the study of an electrode material in lithium-ion batteries, electrochemical testing aims at understanding the dynamics of $\mathrm{Li}^{+}$moving into and out of the electrode, which determines its performance in terms of capacity, retention, and rate capability.

Two basic techniques are generally used in electrochemical investigations: galvanostatic cycling (GC) and cyclic voltammetry (CV). The GC method is based on a chronopotentiometry action, where a fast-rising current pulse is enforced on the working electrode of an electrochemical cell and maintained at a constant level, while the potential of this electrode is measured against a reference electrode as a function of time, as schematically shown in Figure 2.5. The direction of the current is determined by the charging or discharging action of the cell. For a continuous cycling, a number of chronopotentiometry actions are connected in sequence, reversing the direction of the current at the beginning of each section, with the amplitude of the current unchanged. Using this technique, the actual capacity of a battery at that specific current, which is more often referred to as the charge/discharge rate, can be obtained by the product of the imposed current and the elapsed time between the beginning and the end of a charge or discharge process. For instance, the $x$-axis in Figure 2.5(b) can be converted to discharge capacity of the battery through multiplying it by the current amplitude. Many researchers adopt a formation cycle method in GC experiments, where the first few cycles, usually at a very slow rate, were performed for the organic electrolytes to decompose and for the solid-electrolyte interphase (SEI) to form, before starting data acquisition on any measurement of interest. This is because the charges passed during these processes are irreversible, resulting in erroneously higher capacity than what can actually be repeated.

In $\mathrm{CV}$, the potential of a battery is controlled by applying a changing voltage on the cell, which is a triangular function with respect to time, and the resulting current is measured. It is known that current peaks appear in both cathodic and anodic scan direction when an electrochemically reversible system is considered. The waveform of the applied voltage and a 
typical CV curve is shown in Figure 2.6. The peak positions [V, Figure 2.6(b)] indicate the potential where redox reactions occur, i.e. when $\mathrm{Li}^{+}$is inserted into or extracted from the electrode, which is the critical information in understanding a battery material. In some circumstances, a hold may be desired at the end of the voltage ramp [vertex hold in Figure 2.6(a)], such as to allow a full lithiation or delithiation in the electrode material.

The scan rate (or sweep rate), i.e. the slope of the triangular wave in CV [Figure 2.6(a)], defined as $v=\mathrm{d} E / \mathrm{dt}$ and usually used with the unit $\mathrm{mV} / \mathrm{s}$, is closely tied to the electrochemical process in a particular material of interest. For a strictly diffusion-limited irreversible redox reaction, the current in amperes at the peak of the $\mathrm{CV}$ curve scales with the square root of the scan rate $v$, as described by: ${ }^{16}$

$$
|i|=0.4958 n F A c\left(\frac{D \alpha n F}{R T}\right)^{1 / 2} v^{1 / 2}
$$

where $i$ is the peak current, $n$ is the number of electrons, $A$ is the electrode area in $\mathrm{cm}^{2}, c$ is the maximum concentration of $\mathrm{Li}^{+}$in the accumulation layer, $D$ is the chemical diffusion coefficient for $\mathrm{Li}^{+}$in $\mathrm{cm}^{2} / \mathrm{s}$, and $\alpha$ is the transfer coefficient usually taken as $0.5 .^{58}$ A capacitive current, on the other hand, normally follows a linear dependence on the scan rate: ${ }^{58}$

$$
\left|i_{C}\right|=A C_{d} v
$$

where $i_{c}$ and $C_{d}$ are the capacitive current and the double-layer capacitance, respectively. $\mathrm{TiO}_{2}-\mathrm{B}$ is particularly attractive as a battery material because its charge storage is not limited by bulk diffusion, but instead by surface processes, so that the overall behavior seems capacitive, exhibiting a linear dependence of the current on the scan rate. ${ }^{16,17}$

\subsubsection{Apparatus and procedure}

The bronze films in this study were assembled in the so-called battery half-cells, where lithium metal was used as counter electrode with a $1.55 \mathrm{~mm}$ thick glass fiber separator between the cathode (film) and anode (lithium). Non-aqueous $\mathrm{LiPF}_{6}$ solution was adopted as electrolyte, either homemade (1M LiPF 6 in ethylene carbonate:dimethyl carbonate 1:1 (v/v), Merck), or 
commercially purchased (Sigma-Aldrich).

A typical bronze film in the electrochemical testing was grown on a $10 \times 10 \mathrm{~mm}^{2}$ substrate with a thickness of 50-200 $\mathrm{nm}$. Considering the very small mass of the active material, which is on the order of a few tens of $\mu \mathrm{g}$, a larger sample is preferred to reduce experimental error. Commercial cells (EL-CELL ECC-STD) which can accommodate samples up to $18 \mathrm{~mm}$ in diameter were assembled in an argon-filled glove box (Innovative Technology Inert Lab) with $\mathrm{O}_{2}$ and $\mathrm{H}_{2} \mathrm{O}$ levels below 2 and $1 \mathrm{ppm}$, respectively, and tested at room temperature on a Princeton Applied Research VersaSTAT MC 4-channel system operating in galvanostatic mode. The structure of the cell and the testing equipment are shown in Figure 2.7. To ensure a steady power supply to the system during long term cycling, a UPS (uninterruptible power supply) unit is equipped for the controlling computer and the VersaSTAT.

The VersaSTAT system employs a five-lead connection geometry. For most investigations into batteries, capacitors, resistors, fuel cells and some sensors, a two-terminal connection is used, where the working electrode (WE, the material of interest) lead and the sense electrode (SE, measures/controls the voltage against the reference electrode) lead are both connected to the material at which the desired reactions will occur, while the counter electrode (CE) lead and the reference electrode (RE, voltage control against SE) lead are both connected to the material used as counter electrode (e.g. lithium). The fifth lead is the ground lead and not ordinarily used in most experiments. The two-terminal configuration is schematically shown in Figure 2.8(a). A three-terminal connection is used for general aqueous electrochemistry, corrosion experiments, and most EIS (electrochemical impedance spectroscopy) experiments, as shown in Figure 2.8(b). The voltage at WE/SE is controlled relatively to a stable reference electrode positioned in close proximity to the working electrode. All battery cells in this study were connected in the two-terminal configuration.

\subsubsection{Data acquisition and processing}

The electrochemical testing data on the $\mathrm{Ca}: \mathrm{TiO}_{2}-\mathrm{B}$ and $\mathrm{TiO}_{2}-\mathrm{B}$ thin films were collected 
in the VersaStudio software bundled with the VersaSTAT system. The software offers a wide variety of basic operations, which can be programmed in sequences or loops to perform complicated electrochemical experiments.

To set up a GC experiment, one should first estimate the $1 \mathrm{C}$ current for the sample of interest. For example, $\mathrm{Ca}: \mathrm{TiO}_{2}-\mathrm{B}$ has a theoretical density of $3.637 \mathrm{~g} \mathrm{~cm}^{-3}$ and a specific capacity of $294 \mathrm{~mA} \mathrm{~h} \mathrm{~g}^{-1}$ (see Sections $3.5 \& 4.3$ ), so a $53 \mathrm{~nm}$ thick $\mathrm{Ca}: \mathrm{TiO}_{2}-\mathrm{B}$ film grown on a $5 \times 5 \mathrm{~mm}^{2}$ substrate has a theoretical capacity of:

$$
53 \times 10^{-7} \mathrm{~cm} \times 0.5 \mathrm{~cm} \times 0.5 \mathrm{~cm} \times 3.637 \frac{\mathrm{g}}{\mathrm{cm}^{3}} \times 294 \frac{\mathrm{mAh}}{\mathrm{g}}=1.41 \times 10^{-3} \mathrm{mAh}
$$

Therefore the $1 \mathrm{C}$ current, at which the film completely discharges its capacity in 1 hour, is 1.41 $\mu \mathrm{A}$ for this sample. Figure 2.9 shows the on-screen setup of a continuous cycling performed on such a $\mathrm{Ca}: \mathrm{TiO}_{2}-\mathrm{B}$ film at a $10 \mathrm{C}$ rate, where the current is fixed at $14.1 \mu \mathrm{A}$, in a voltage window of 1-3 V. It is customary to record 100-500 data points in each half-cycle (charge or discharge), so the "Time Per Point" value is set at $1 \mathrm{~s}$ in this example for $10 \mathrm{C}$ cycling. If the rate is much lower, such as $\mathrm{C} / 10$, the device is often programmed to record one data point every $10 \mathrm{~s}$ or longer. It is worth noting that the $\mathrm{C}$ unit is defined by the theoretical capacity of the material, which is usually not reached in experiments, so the time the sample actually takes to fully discharge its capacity at 10C might not be exactly $360 \mathrm{~s}(1 \mathrm{hr} / 10)$, but shorter.

The raw data recorded in a GC experiment is in the form of potential (V) vs. elapsed time (s). A simple script on common programming platforms such as Matlab or Origin can be used to sift through the data stream and pick out all the end points of each half-cycle, three of which are marked in Figure 2.9 as $t_{\mathrm{n}-1}, t_{\mathrm{n}}$ and $t_{\mathrm{n}+1}$. Considering a battery half-cell with metallic Li counter electrode, from $t_{\mathrm{n}-1}$ to $t_{\mathrm{n}}, \mathrm{Li}$ ions are inserted into the $\mathrm{Ca}: \mathrm{TiO}_{2}-\mathrm{B}$ thin film, lowering the cell voltage, which is a discharge process; and inversely from $t_{\mathrm{n}}$ to $t_{\mathrm{n}+1}$, a charge process. The battery capacity in each half cycle is calculated via multiplying the respective time period $\left(t_{\mathrm{n}}-t_{\mathrm{n}-1}\right.$ and $t_{\mathrm{n}+1}-t_{\mathrm{n}}$ ) by the current, which is then divided by the mass of the film to obtain specific capacity. The Coulombic efficiency is defined as: 


$$
\text { Coulombic efficiency }=\frac{\text { Charges that can be extracted }}{\text { Charges that enter the battery }}
$$

which can be calculated by the ratio between the discharge capacity and the charge capacity, is an important index to gauge the cyclability of a battery material. A Coulombic efficiency closer to 1 , which suggests almost all $\mathrm{Li}^{+}$inserted into the active material can be extracted, is desired in long term cycling. The charge and discharge capacities, along with the Coulombic efficiency, at each cycle are often plotted vs. the cycle number, e.g. Figure 4.13.

In order to create an intuitive comparison between different cycles or between different rates, it is conventional to shift the charge curve in a particular cycle, i.e. the curve from $t_{\mathrm{n}}$ to $t_{\mathrm{n}+1}$ in Figure 2.9 , to the left so $t_{\mathrm{n}}$ lines up with $t_{\mathrm{n}-1}$, and convert the $x$-axis to capacity (or charge passed/Li content). This way, the voltage profiles (sometimes called load curves) in multiple cycles can be overlapped in the same plot to demonstrate capacity retention (e.g. Figure 6.5); or those with the same cycle number but at multiple rates to demonstrate rate capabilities (e.g. Figure 5.5). Many researchers also adopt a modified version of this plot, where the charge curve is mirrored horizontally and the end point of the discharge curve still meets the start point of the charge curve. In Figure 2.9, this means $t_{\mathrm{n}}$ is fixed while flipping the curve from $t_{\mathrm{n}}$ to $t_{\mathrm{n}+1}$ to the left. The purpose of such a modified plot is to clearly illustrate the amount of $\mathrm{Li}$ inserted into the active material that is trapped and cannot be extracted later, i.e. the capacity loss. Most authors show one cycle of this plot (e.g. Figure 4.11), while some draw a few cycles connected together to demonstrate capacity retention (e.g. Ref. 28).

The GC experimental data can be further used to generate a differential capacity $(\mathrm{d} Q / \mathrm{d} V)$ plot, which is obtained by taking a derivative of elapsed time with respect to the potential and multiplying by the current:

$$
\frac{\mathrm{d} Q}{\mathrm{~d} V}=I \frac{\mathrm{d} t}{\mathrm{~d} V}
$$

The differential capacity plot is very sensitive to plateaus in the voltage profiles (small $\mathrm{d} V$ with elapsed time), showing them as peaks. ${ }^{33,36}$ Physically, the peak positions (V) in the $\mathrm{d} Q / \mathrm{d} V$ plot are linked to the potential where redox reactions occur in the electrode material, so they usually correspond well with the $\mathrm{CV}$ testing results. Therefore, these plots are often used when the $\mathrm{CV}$ 
technique is not available, or when the peaks on the CV curves are difficult to identify.

The setup for a CV measurement in the VersaStudio software is more straightforward. As shown in Figure 2.10, one only needs to add a few basic CV actions into the sequence as desired, each set with a specific scan rate $(\mathrm{V} / \mathrm{s})$, the number of cycles and the voltage window. No more data processing is necessary after the experiment finishes, unless curve smoothing is preferred, which can be done by the sampling or smoothing functions available in most data plotting software such as Origin.

The importance of planning should be emphasized in an electrochemical research project, since battery cycling experiments usually take a long time. For instance, slow rate cycling is necessary to probe the thermodynamic equilibrium state of the battery, which is very useful in extracting information on kinetic effects at higher rates; however, at a rate of $\mathrm{C} / 12$, one cycle takes an entire day. In a CV measurement, one cycle between 1 and $3 \mathrm{~V}$ at a scan rate of 0.1 $\mathrm{mV} / \mathrm{s}$ takes more than 11 hours. Hence, a complete set of experiments at various rates on one sample often need weeks or even months to finish. The researcher should fully utilize the multi-channels on the testing device and arrange more samples to be tested at the same time. 


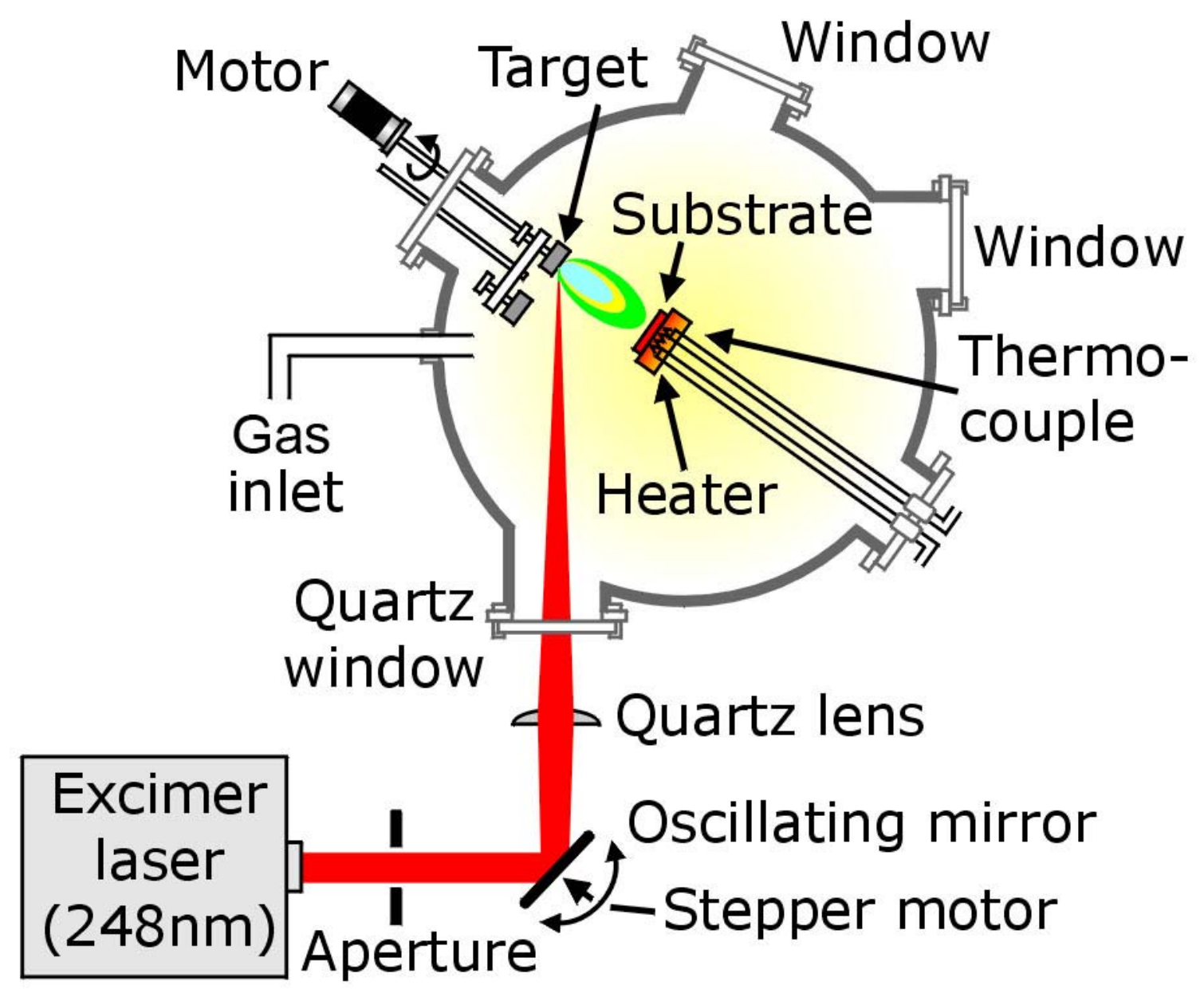

Figure 2.1 Schematic of a typical PLD system (reproduced and modified from a schematic drawing available on http://www.egr.msu.edu/). 


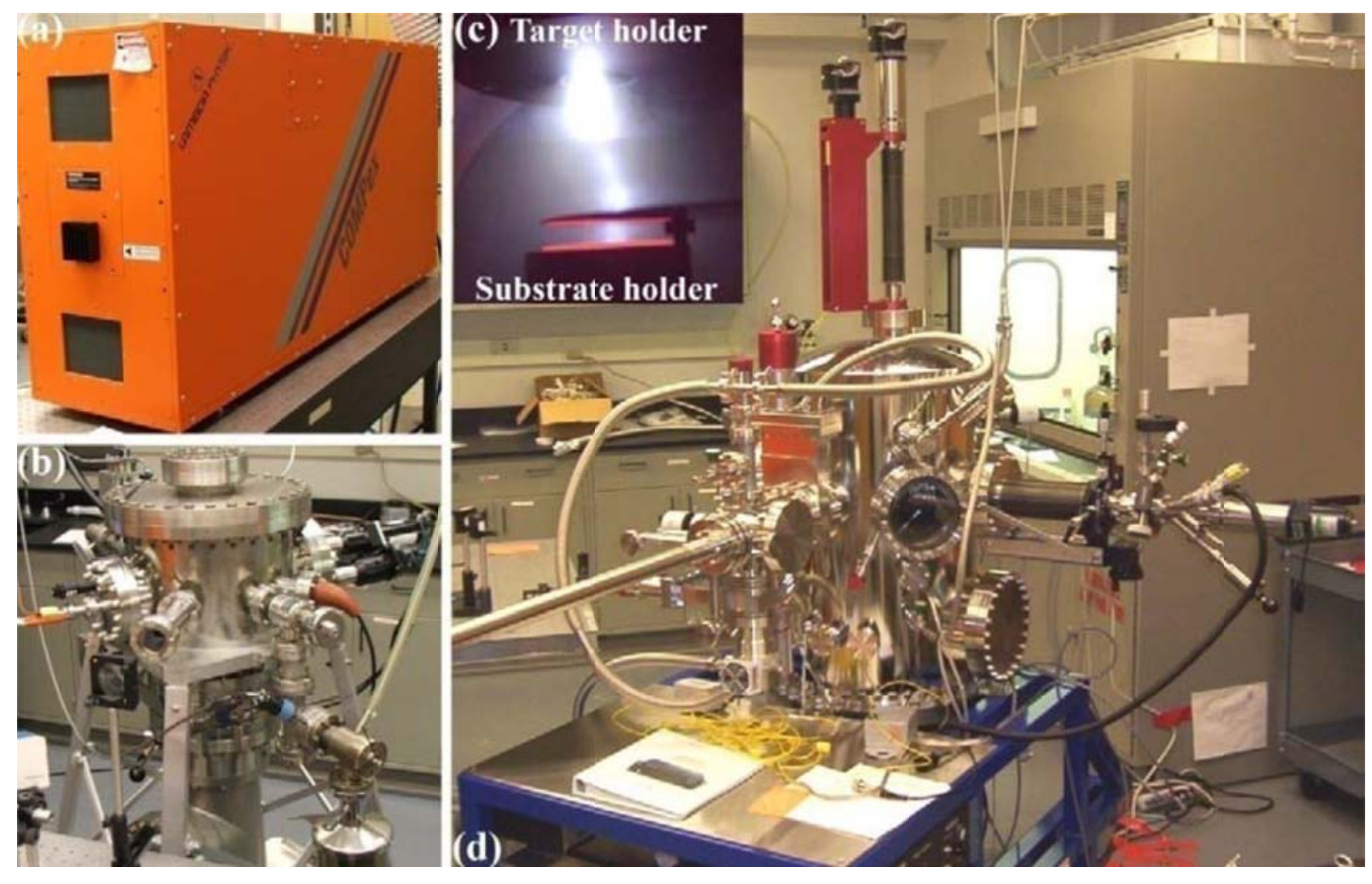

Figure 2.2 Photographs of (a) the excimer laser (COMPex 205), (b) the Neocera PLD system, (c) the laser plume captured in the Neocera system, and (d) the Thermionics system. This figure is reproduced from Ref. 59. 


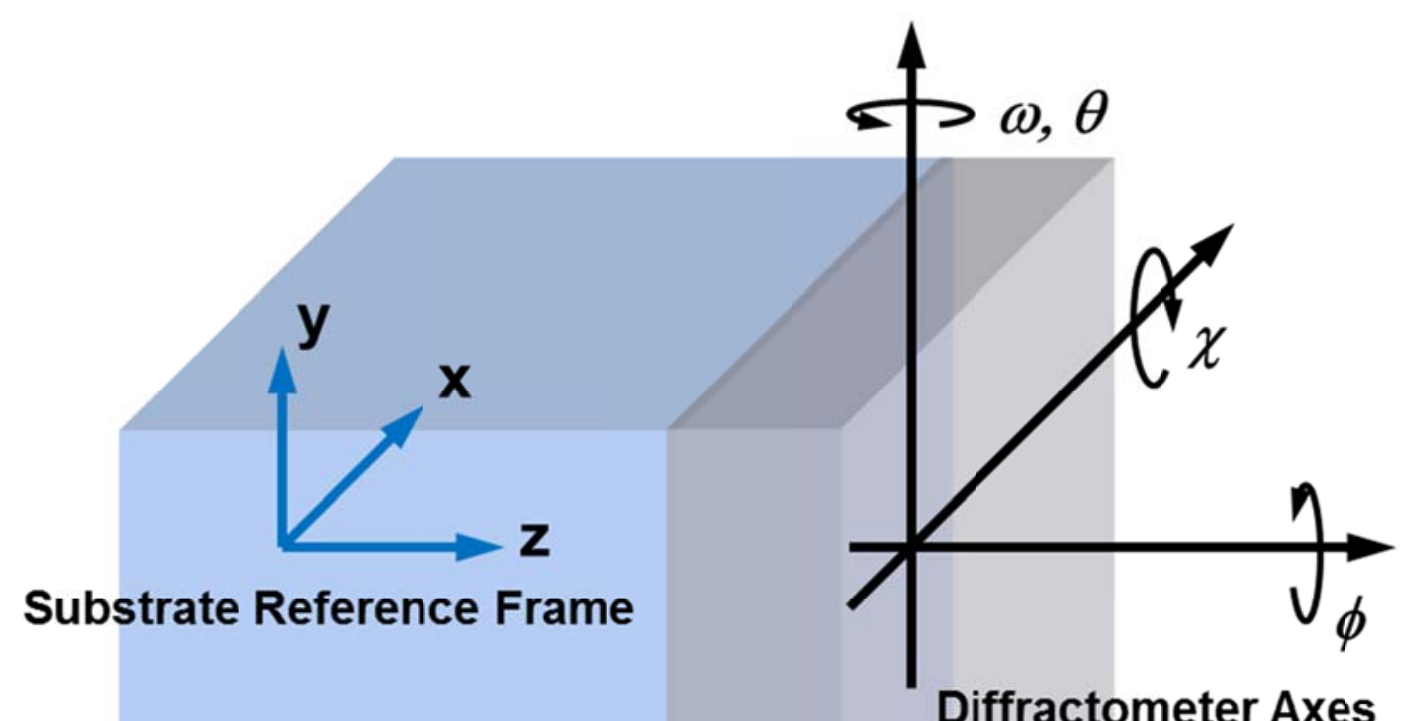

\section{Film}

\section{Substrate}

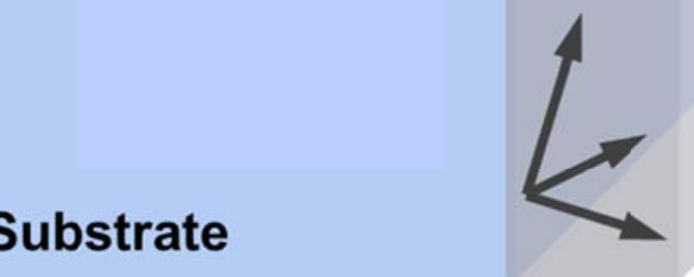

\section{Crystallite Reference Frame}

Figure 2.3 Schematic showing the relationship between the diffractometer axes and the substrate reference frame in the XRD measurements (modified from Ref. 60). 


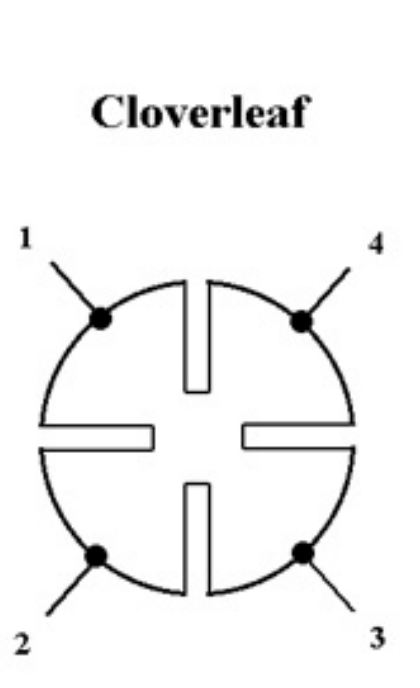

(a)

Preferred
Square or rectangle:

contacts at

the corners

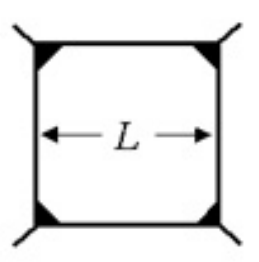

(b)

Acceptable
Square or rectangle:

contacts at the edges or inside the perimeter

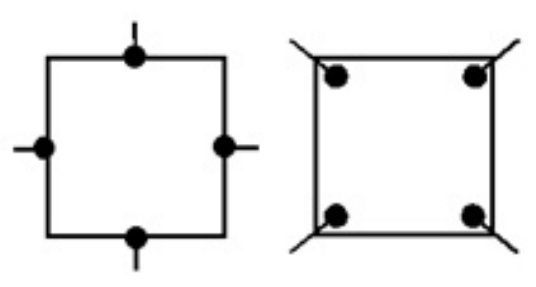

(c)

Not Recommended

Figure 4

Figure 2.4 Sample geometries for Van der Pauw resistivity and Hall effect measurements. The cloverleaf design will have the lowest error due to its smaller effective contact size, but it is more difficult to fabricate than a square or rectangle [from NIST, Ref. 56]. The configuration used in this study is (b). 

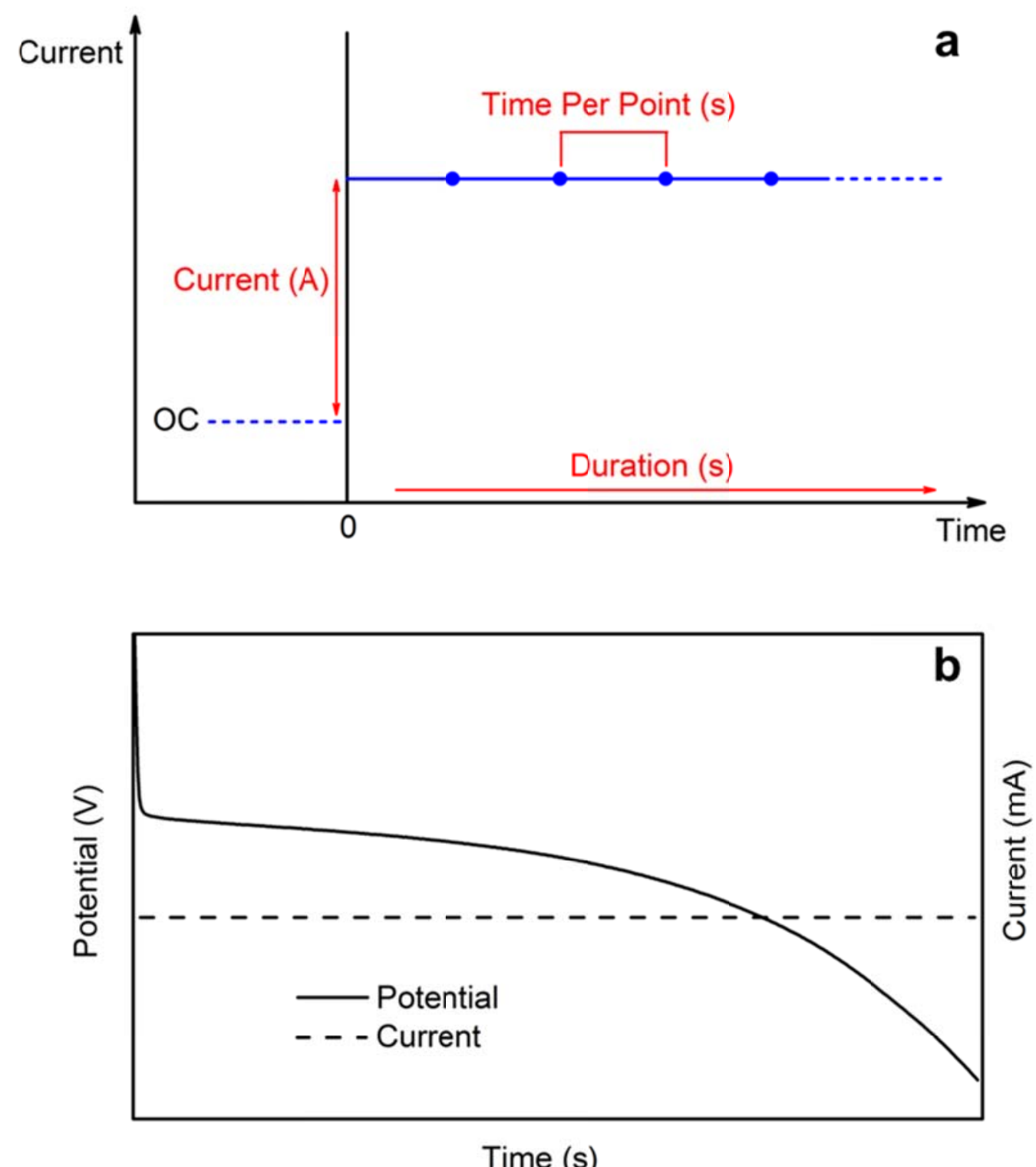

Figure 2.5 (a) The current applied on the battery cell in a galvanostatic cycling (GC) experiment [from the VersaStudio software]. (b) Typical current and potential profiles of a battery in a GC experiment. 

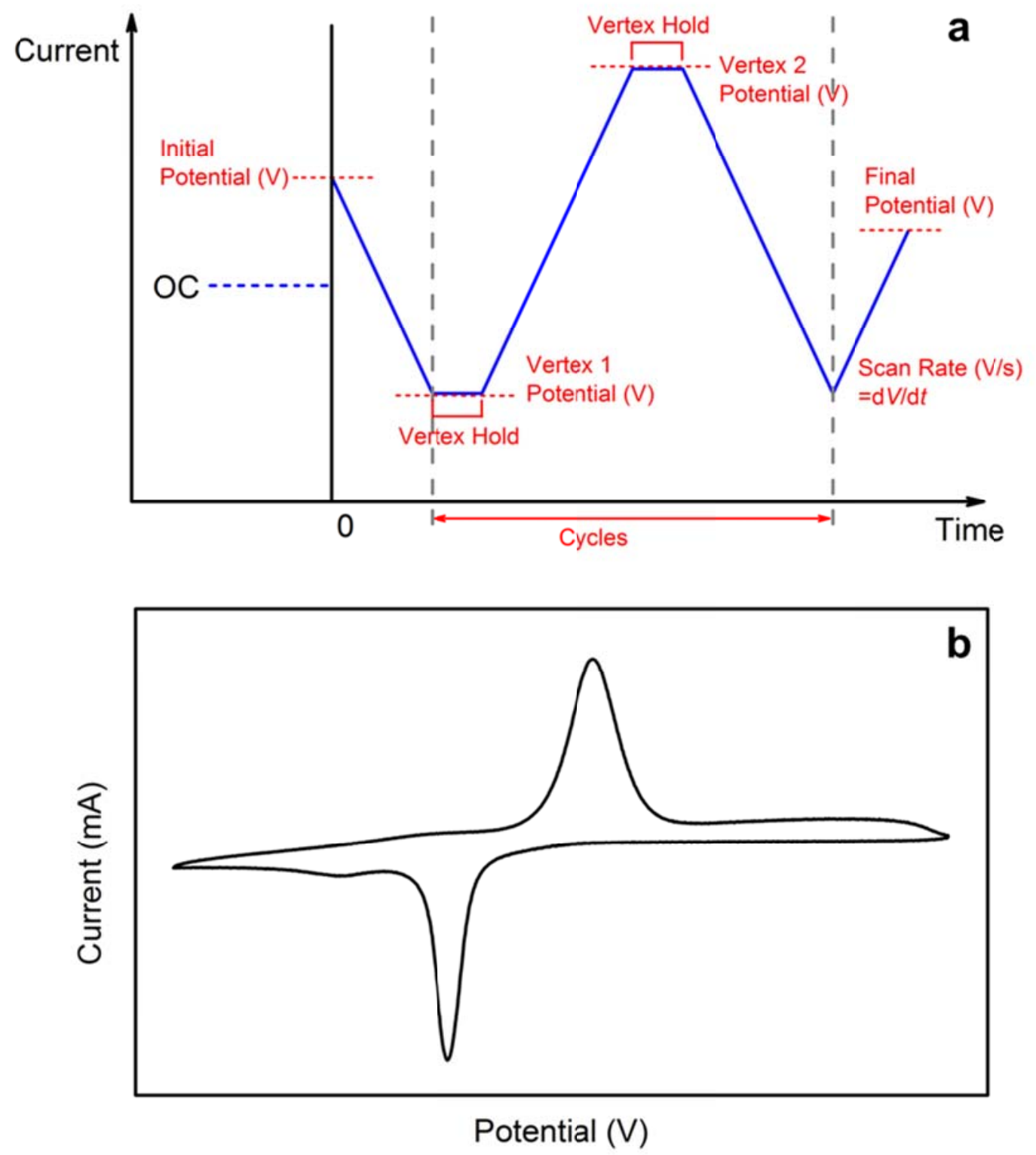

Figure 2.6 (a) The voltage applied on the battery cell in a cyclic voltammetry (CV) experiment [from the VersaStudio software]. (b) A typical CV curve of a battery in a CV experiment. 

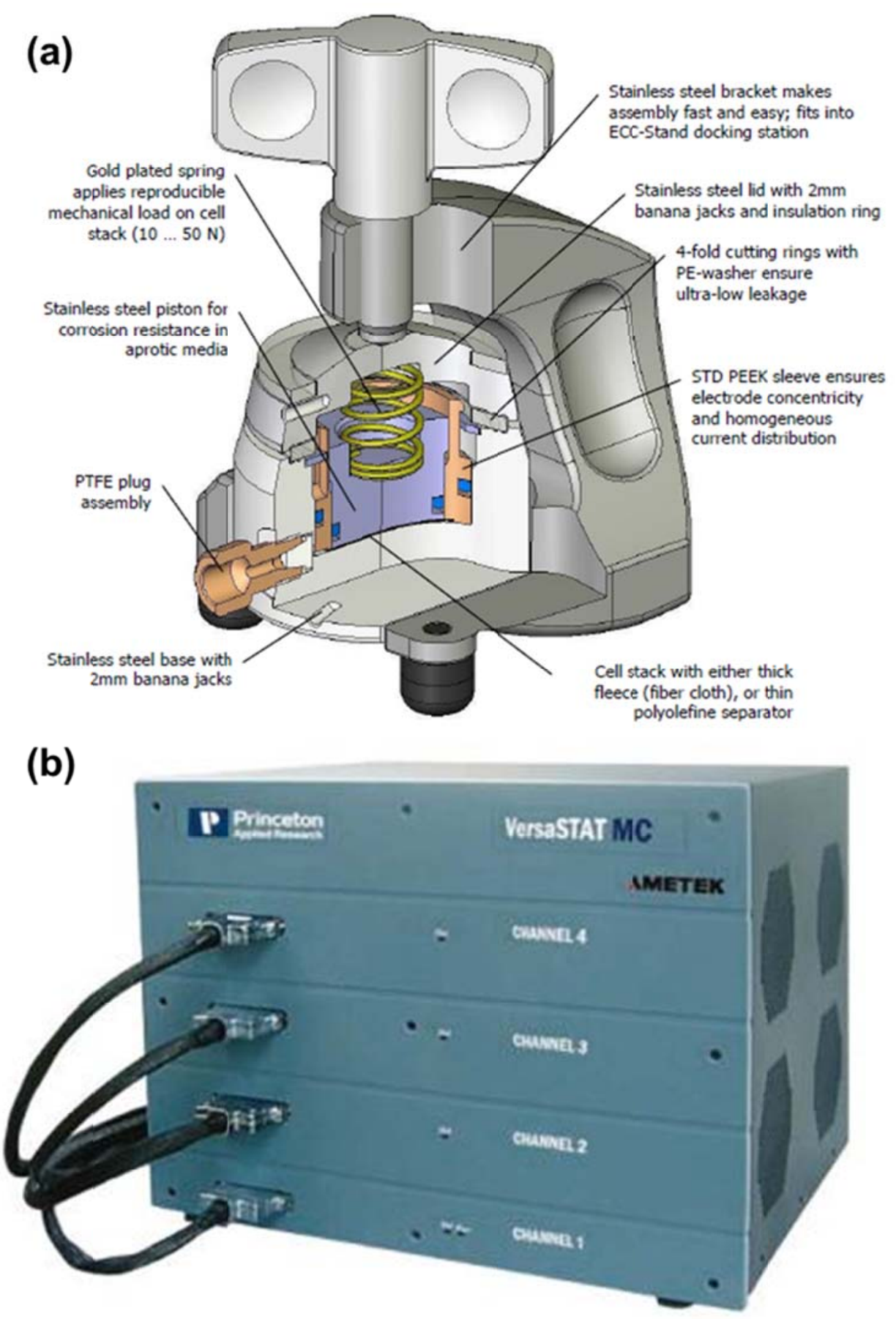

Figure 2.7 (a) Internal structure of the commercial battery test cell (EL-CELL ECC-STD, image credit: http://el-cell.com/). (b) A photograph of the VersaSTAT MC 4-channel electrochemical testing system. [Princeton Applied Research: user manual] 

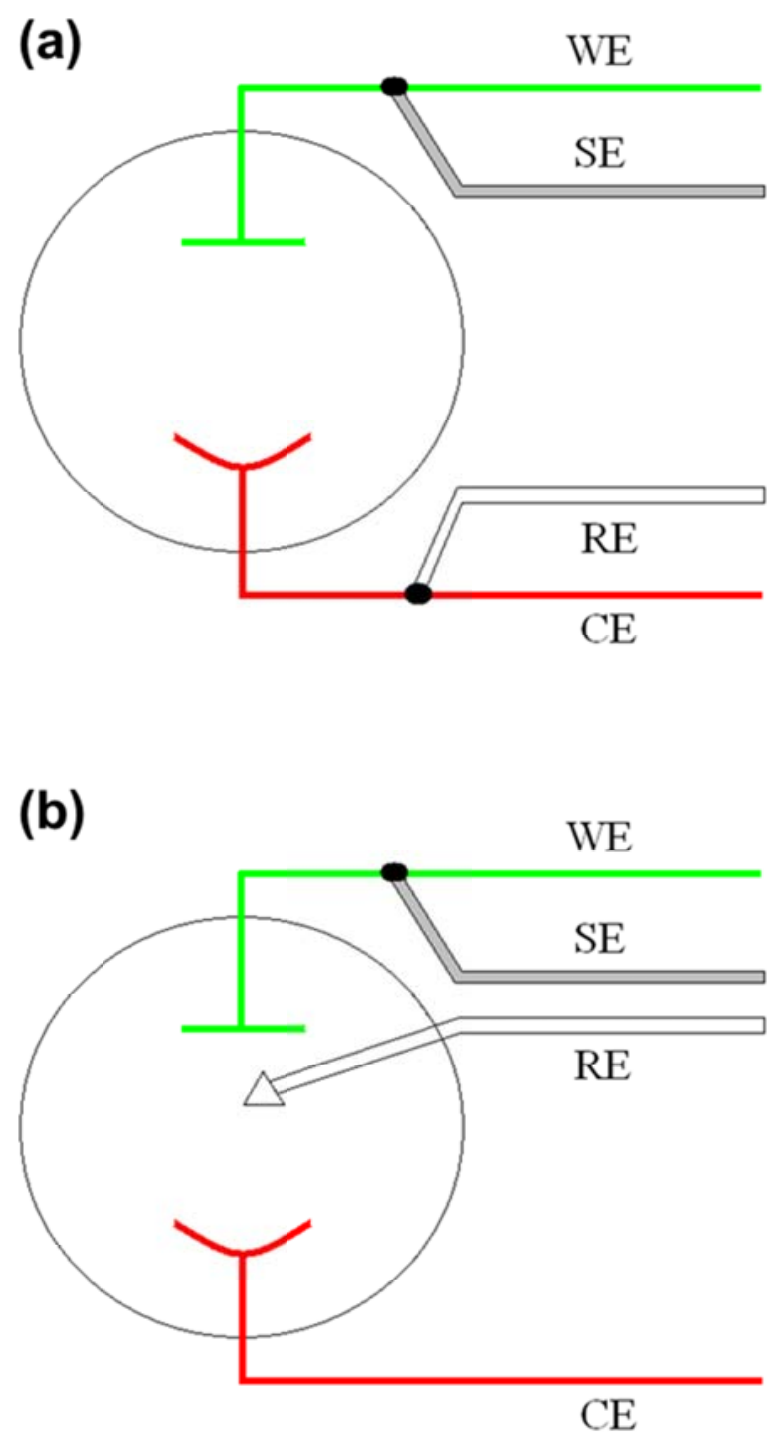

Figure 2.8 (a) The two-terminal connection. (b) The three-terminal connection. [Princeton Applied Research: user manual] 


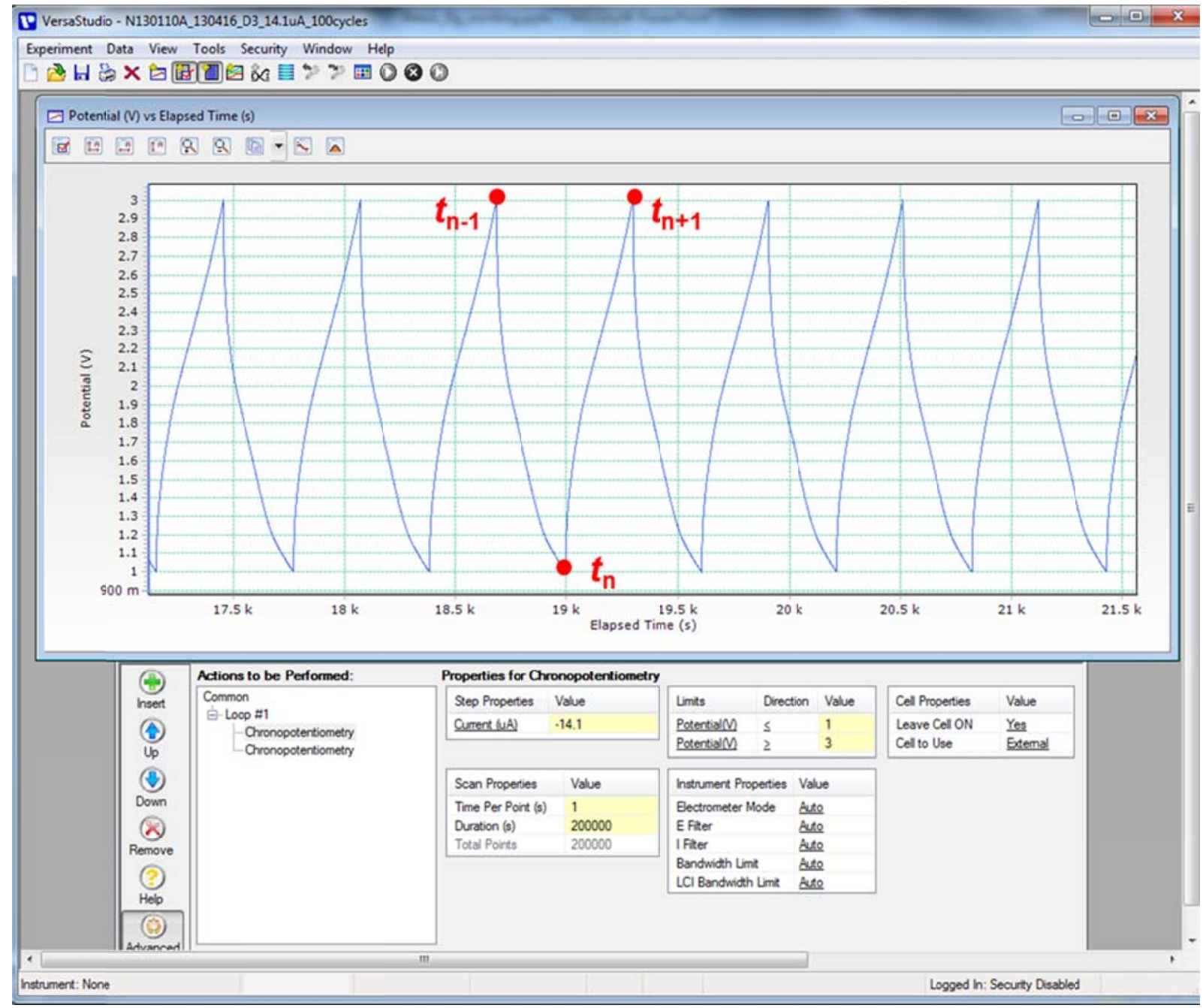

Figure 2.9 Screenshot of the VersaStudio software in a galvanostatic cycling experiment on a (001) $\mathrm{Ca}: \mathrm{TiO}_{2}-\mathrm{B}$ thin film. The current is set at $14.1 \mu \mathrm{A}$, and the voltage window is $1-3 \mathrm{~V}$. 


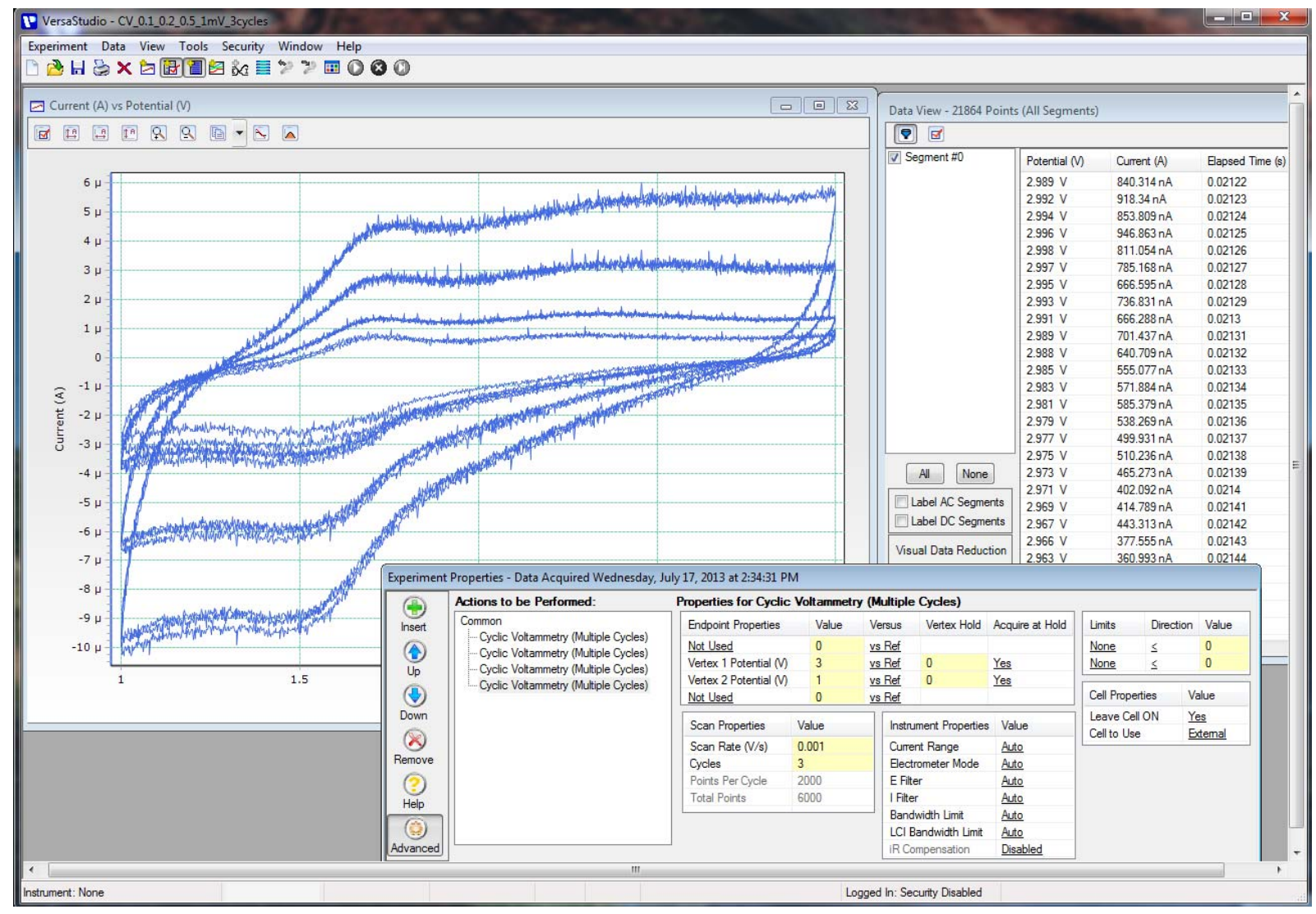

Figure 2.10 Screenshot of the VersaStudio software in a cyclic voltammetry experiment on a (001) $\mathrm{TiO}_{2}-\mathrm{B}$ thin film. The voltage window is $1-3 \mathrm{~V}$. 


\section{Chapter 3}

\section{Epitaxial Growth of High Quality Ca: $\mathrm{TiO}_{2}-\mathrm{B}\left(\mathrm{CaTi}_{5} \mathrm{O}_{11}\right)$ and $\mathrm{TiO}_{2}-\mathrm{B}$ Thin Films}

Being a metastable polymorph, phase pure $\mathrm{TiO}_{2}-\mathrm{B}$ is extremely hard to obtain with good crystallinity, significantly impeding its development both in the fundamental understanding and in potential applications. This chapter demonstrates, in great detail, a new waterless synthesis route to produce single-crystalline epitaxial thin films of $\mathrm{TiO}_{2}-\mathrm{B}$ and its more stable variant $\mathrm{Ca}: \mathrm{TiO}_{2}-\mathrm{B}\left(\mathrm{CaTi}_{5} \mathrm{O}_{11}\right)$, using pulsed laser deposition (PLD). The growth mechanism and various microstructures in the thin films are clearly shown at the atomic scale. These findings can serve as guidelines for creating these materials in a controlled form, providing well-defined lattice orientation and surfaces for future research efforts.

\subsection{Introduction}

The first synthesis of $\mathrm{TiO}_{2}-\mathrm{B}$ dates back to 1980 by Marchand et al. from the layered titanate $\mathrm{K}_{2} \mathrm{Ti}_{4} \mathrm{O}_{9}$ which was converted to $\mathrm{H}_{2} \mathrm{Ti}_{4} \mathrm{O}_{9}$ via acid washing and finally dehydrated to the layered $\mathrm{TiO}_{2}-\mathrm{B}$ structure. ${ }^{7}$ The sample was heated to $500{ }^{\circ} \mathrm{C}$ to remove $\mathrm{H}_{2} \mathrm{O}$, although it was not clear if the removal was complete, as water residual was still likely to be trapped in the structure.

After more than 30 years, hydrothermal methods are still the dominate route to synthesize this material, despite the variations in process parameters and the morphology of the end product. $\mathrm{TiO}_{2}-\mathrm{B}$, and titania in general suffers from poor electrical and ionic conductivity. Therefore, recent research has been focused on nanostructuring, which can help alleviate problems with 
poor ionic conductivity by shortening lithium diffusion pathways. ${ }^{38}$ Kogure et al. (1999) identified $\mathrm{TiO}_{2}-\mathrm{B}$ nanocrystallites of $5-10 \mathrm{~nm}$ in size in a sol-gel derived $\mathrm{SiO}_{2}-\mathrm{TiO}_{2}$ film, prepared by mixing acid-hydrolyzed silicon tetraethoxide, $\mathrm{Si}(\mathrm{OEt})_{4}$, in ethanol with titanium tetra- $n$-butoxide, $\mathrm{Ti}\left(\mathrm{O}^{\mathrm{n}} \mathrm{Bu}\right)_{4}{ }^{19}$ The Bruce group at University of St. Andrews, UK, has successfully synthesized various $\mathrm{TiO}_{2}-\mathrm{B}$ nanostructures, including nanowires (2004) with a typical diameter of $20-40 \mathrm{~nm}$ and a length of 2-10 $\mu \mathrm{m}$, prepared by adding $\mathrm{TiO}_{2}$-anatase to a $15 \mathrm{M}$ aqueous solution of $\mathrm{NaOH}$ at a reaction temperature of $170{ }^{\circ} \mathrm{C}$, and washing with dilute $\mathrm{HCl}$ to promote complete exchange of $\mathrm{Na}^{+}$by $\mathrm{H}^{+}$for the formation of hydrogen titanates; ${ }^{20}$ nanotubes (2004, 2005) with external diameters of 10-20 nm, internal diameters of 5-8 nm, and a length up to $1 \mu \mathrm{m}$, prepared by a similar procedure but using $10 \mathrm{M} \mathrm{NaOH}$ solution at a reaction temperature of $150{ }^{\circ} \mathrm{C}$, which easily convert to anatase upon heating, losing the tubular morphology; ${ }^{20,21}$ and nanoparticulate $\mathrm{TiO}_{2}-\mathrm{B}$ (2012), where the nanoparticles of ca. $2.5 \times 4.3 \mathrm{~nm}$ in size form agglomerates of 0.3-3 $\mu \mathrm{m}$, synthesized by dissolving Ti metal in a mixture of $\mathrm{H}_{2} \mathrm{O}_{2}$ and $\mathrm{NH}_{3}$ in water, adding glycolic acid and finally hydrothermal treatment and calcination. ${ }^{36}$ Yang et al. (2009) fabricated $\mathrm{TiO}_{2}-\mathrm{B}$ nanofibers covered with a 10-20 nm shell of anatase nanocrystals, converted from $\mathrm{H}_{2} \mathrm{Ti}_{3} \mathrm{O}_{7}$ nanofibers by hydrothermal reaction and heating. ${ }^{61}$ Beuvier et al. (2010) synthesized non-porous nanoribbons around $30 \mathrm{~nm}$ large and $1-2 \mu \mathrm{m}$ long by refluxing treatment, ionic exchange and thermal treatment of the titanic acid. ${ }^{22}$ Liu et al. (2011) designed mesoporous $\mathrm{TiO}_{2}-\mathrm{B}$ microspheres comprising of micrometer-sized particles with a diameter of around $1 \mu \mathrm{m}$, uniform mesopores of $12 \mathrm{~nm}$, and nanosized crystal grains of $\sim 6 \mathrm{~nm}$, prepared by a template assisted ultrasonic spray pyrolysis method followed by refluxing, ion-exchange and heat treatment. ${ }^{23}$ Zhou et al. (2011) synthesized $\mathrm{TiO}_{2}$ nanobelts by annealing hydrothermally acquired $\mathrm{H}_{2} \mathrm{Ti}_{3} \mathrm{O}_{7}$ nanobelts at different temperatures, resulting in different $\mathrm{TiO}_{2}$ phases including $\mathrm{TiO}_{2}-\mathrm{B}$, where the nanobelts were typically 20-40 nm thick, 50-200 nm wide, and up to several tens of $\mu \mathrm{m}$ long. ${ }^{25}$ Liu et al. (2012) presented a one-step preparation of porous $\mathrm{TiO}_{2}$-B constructed by nanosheets with 5-10 nm thickness, $3.6 \mathrm{~nm}$ pore size, and petal-like morphology, based on the self-assembly of titanium precursor chelated by $\mathrm{TiCl}_{4}$ and ethylene glycol and the subsequent 
solvothermal condensation in the presence of ammonia. ${ }^{24}$ Liu et al. (2013) reported an additive-free flexible film electrode by anchoring $\mathrm{TiO}_{2}-\mathrm{B}$ nanosheets on non-woven activated carbon fabric, where the 30-40 $\mathrm{nm}$ thick nanosheets were deposited by kinetically controlled hydrolysis and condensation of the titanium precursor. ${ }^{35}$ Etacheri et al. (2013) synthesized highly mesoporous nanosheet-assembled hierarchical $\mathrm{TiO}_{2}-\mathrm{B}$ microflowers, where the process involved the corrosion of Ti-foil in alkaline hydrogen peroxide solution under hydrothermal condition, resulting in microflowers that have a spherical shape with an average diameter of $1.5 \mathrm{~mm}$, composed of 5-10 nm thick bundled 2-D nanosheets that uniformly grow perpendicular to the center forming a hierarchical morphology. ${ }^{26}$

In these representative reports on the $\mathrm{TiO}_{2}-\mathrm{B}$ material summarized above, nanostructuring has indeed brought about excellent photocatalytic and battery performances, where the discharge capacity is generally higher than $270 \mathrm{~mA} \mathrm{~h} \mathrm{~g}^{-1}$ and up to $332 \mathrm{~mA} \mathrm{~h} \mathrm{~g}^{-1}\left(\mathrm{Li}_{0.99} \mathrm{TiO}_{2}\right)$. However, the presence of lattice water in the structures prepared by these hydrothermal methods is unavoidable, as the end products are likely to include layered hydrogen titanates $\mathrm{H}_{2} \mathrm{Ti}_{n} \mathrm{O}_{2 n+1} \cdot x \mathrm{H}_{2} \mathrm{O}$, which exhibit features similar to $\mathrm{H}_{2} \mathrm{Ti}_{3} \mathrm{O}_{7}, \mathrm{H}_{2} \mathrm{Ti}_{4} \mathrm{O}_{9} \cdot \mathrm{H}_{2} \mathrm{O}$, and other members of the hydrogen titanate family. ${ }^{20,21}$ Residual $\mathrm{H}_{2} \mathrm{O}$ in the structure may interfere with $\mathrm{Li}^{+}$ transport, lowering charge storage capacity, rate capability and capacity retention, or react with $\mathrm{Li}$, causing instability to the battery. More significantly, recent studies have suggested that $\mathrm{H}_{2} \mathrm{O}$ may even be needed to keep the bronze structure obtained in this route from collapsing into anatase upon aggressive heating. ${ }^{20,26,38,39}$ Additionally, the precursors, which often times contain other more stable $\mathrm{TiO}_{2}$ polymorphs such as anatase or rutile, rarely fully react, also contributing to the limited phase purity for $\mathrm{TiO}_{2}-\mathrm{B}$.

Furthermore, in spite of the extensive efforts to synthesize this material, especially in the past decade, a true bulk of $\mathrm{TiO}_{2}-\mathrm{B}$ is still lacking, largely due to the fact that it is a metastable phase. Though many researchers have used a "bulk" $\mathrm{TiO}_{2}-\mathrm{B}$ sample in property tests to contrast the electrochemical dynamics in nanostructure-engineered electrodes, ${ }^{20,21,36}$ the "bulk" actually stands for large powder particles, or a mechanically pressed, possibly sintered, pellet made from 
powders, which mimic a longer $\mathrm{Li}^{+}$transport pathway. Apparently, this is quite different from the strict definition of bulk, which refers to a relatively large single crystal. The grain boundaries between the powder particles, or the confinement effects depending on the particle sizes, would all cloud the fundamental understanding of the material, while complicating property testing data analysis because of the lack of a good baseline. Compounded by the random distribution of the nanocrystallites, direct association of test results from these nanostructures with theoretical studies is often difficult, where the latter simulates a perfect crystal, while adding defects and boundaries multiplies the computational complexity exponentially. Specifically for $\mathrm{TiO}_{2}-\mathrm{B}$, or other battery materials that exhibit the attractive pseudocapacitive behavior, a true bulk is critical to distinguish between bulk effects and surface effects.

In light of the above, a new method to synthesize high quality $\mathrm{TiO}_{2}-\mathrm{B}$ crystal is highly desired. Based on first principles calculations, x-ray diffraction (XRD) and atomic resolution high-angle annular dark-field (HAADF) scanning transmission electron microscopy (STEM), ${ }^{62}$ we have observed a stable derivative of the $\mathrm{TiO}_{2}-\mathrm{B}$ structure, $\mathrm{CaTi}_{5} \mathrm{O}_{11}$ (referred to as $\mathrm{Ca}: \mathrm{TiO}_{2}-\mathrm{B}$ below), and have demonstrated that it can be used as an anode material in lithium-ion batteries

with significantly enhanced rate capability via orientation engineering. ${ }^{12}$ In this chapter, we report a thin film growth approach to create high quality new $\mathrm{Ca}: \mathrm{TiO}_{2}-\mathrm{B}$ phase and its role as a template for the waterless synthesis of pure $\mathrm{TiO}_{2}-\mathrm{B}$. We will discuss the optimization of the PLD growth conditions, including target composition, choice of substrates, growth temperature, laser energy and $\mathrm{O}_{2}$ partial pressure, to achieve the best purity and crystallinity for the $\mathrm{Ca}: \mathrm{TiO}_{2}-\mathrm{B}$ and $\mathrm{TiO}_{2}-\mathrm{B}$ phases.

\subsection{First observation of the $\mathrm{Ca}: \mathrm{TiO}_{2}-\mathrm{B}$ phase}

The $\mathrm{TiO}_{2}$ - $\mathrm{B}$ phase was first observed unexpectedly as a defect phase inclusion in $\mathrm{Pt}$ doped $\mathrm{CaTiO}_{3}$ films grown by PLD. A $\mathrm{CaTi}_{0.95} \mathrm{Pt}_{0.05} \mathrm{O}_{3}$ thin film deposited at $750{ }^{\circ} \mathrm{C}$ in $50 \mathrm{mTorr}$ $\mathrm{O}_{2}$ showed good epitaxy as grown on a (100) $\mathrm{SrTiO}_{3}$ substrate, which was homogeneous and comprised a single perovskite phase, without any second phases observed in STEM. When the 
film was exposed to reducing conditions $\left(800{ }^{\circ} \mathrm{C}, 10 \% \mathrm{H}_{2} / \mathrm{N}_{2}, 1 \mathrm{~h}\right)$ in order to induce the extrusion of metallic Pt from the perovskite matrix, ${ }^{63}$ Pt clusters 1-2 $\mathrm{nm}$ in diameter precipitated from the $\mathrm{CaTiO}_{3}$ host, while the oxide itself in the $\mathrm{CaTi}_{0.95} \mathrm{Pt}_{0.05} \mathrm{O}_{3}$ film also underwent significant local phase transformations in the vicinity of the Pt clusters that had nucleated upon reduction. Small 2-15 $\mathrm{nm}$ regions of second phases formed epitaxially to the surrounding perovskite matrix, as shown in Figure 3.1(a). ${ }^{62,64}$ These phases were identified as the anatase phase and $\mathrm{TiO}_{2}-\mathrm{B}$, respectively, by their crystal structure as imaged in HAADF STEM and their stoichiometry - the absence of Ca relative to the surrounding matrix - as probed by EELS. The bronze phase regions are heavily twinned due to the lattice mismatch with the surrounding perovskite.

The $\mathrm{Ca}: \mathrm{TiO}_{2}-\mathrm{B}$ phase was also identified in STEM as another defect phase in the above sample, co-existing with $\mathrm{TiO}_{2}-\mathrm{B}$, as well as in other samples. Figure 3.2(a) shows a defect phase inclusion containing the $\mathrm{Ca}: \mathrm{TiO}_{2}-\mathrm{B}$ structure, where the arrow marks the unique Ti-Ca alternating layer. More generally in this sample, $\mathrm{Ca}: \mathrm{TiO}_{2}-\mathrm{B}$ forms out of the perovskite in small domains of order $10 \mathrm{~nm}$ in diameter, generally with its main crystallographic axes co-directional with those of the surrounding perovskite. Within these domains, however, about $1 / 3$ to $1 / 2$ of the volume is the simple $\mathrm{TiO}_{2}-\mathrm{B}$ structure, of which $\mathrm{Ca}: \mathrm{TiO}_{2}-\mathrm{B}$ is a twinned, augmented variant with a layer comprising one ion each of $\mathrm{Ca}$, $\mathrm{Ti}$, and $\mathrm{O}$ separating each twin plane. It is likely that the small size of the domains along with the large size of the $\mathrm{Ca}: \mathrm{TiO}_{2}-\mathrm{B}$ unit cell and its existing crystallographic template allows the otherwise slightly more unfavorable $\mathrm{TiO}_{2}$ - $\mathrm{B}$ to easily form. The dashed line in Figure 3.2(a) marks the interface between the two bronze phases. Such templating effect has been magnified to create high quality single-crystalline $\mathrm{TiO}_{2}-\mathrm{B}$ epilayers on top of a $\mathrm{Ca}: \mathrm{TiO}_{2}-\mathrm{B}$ template layer, as will be shown later in this chapter. Even larger $\mathrm{Ca}: \mathrm{TiO}_{2}-\mathrm{B}$ grains were observed in a $\mathrm{Rh}$ doped $\mathrm{CaTiO}_{3}\left(\mathrm{CaTi}_{0.95} \mathrm{Rh}_{0.05} \mathrm{O}_{3}\right)$ thin film under the same growth and thermal treatment conditions as the above Pt doped film, an example of which is shown in Figure 3.2(b) adjacent to a $\mathrm{Rh}$ particle precipitate. It is not yet clear whether the Pt and $\mathrm{Rh}$ had any catalysis effect during the phase formation of $\mathrm{TiO}_{2}-\mathrm{B}$ and $\mathrm{Ca}: \mathrm{TiO}_{2}-\mathrm{B}$ in these samples. 
Based on the experimental results, theoretical simulation of phase evolution was focused on temperatures between $100{ }^{\circ} \mathrm{C}$ and $800{ }^{\circ} \mathrm{C}$, mirroring those accessible during our experiments, and in oxygen partial pressures $\left(P_{O_{2}}\right)$ from 0.2 to $1 \times 10^{-8}$ atm (152 Torr to 0.0076 mTorr), representing oxidizing and reducing conditions, respectively. Pseudo-ternary phase diagrams were calculated by minimizing the grand canonical free energies of all compounds reported to exist within the Ca-Ti-Pt-O system. ${ }^{62}$ Phase diagrams were calculated at intervals of $100{ }^{\circ} \mathrm{C}$ between $100{ }^{\circ} \mathrm{C}$ and $1200{ }^{\circ} \mathrm{C}$ for $P_{O_{2}}=0.2$ atm and between $100{ }^{\circ} \mathrm{C}$ and $800{ }^{\circ} \mathrm{C}$ for $P_{O_{2}}=1 \times 10^{-8}$ atm. A selection of these calculated phase diagrams are shown in Figure 3.1 representing moderate redox conditions, while the full set of calculation results can be found in Ref. 62, in which the $\mathrm{Ca}: \mathrm{TiO}_{2}-\mathrm{B}$ phase always exists until it disappears at $1 \times 10^{-28}$ atm, an extremely aggressive reducing condition, suggesting that it is a thermodynamically stable phase.

Guided by these initial observations and theoretical predications, a systematic study to grow phase pure $\mathrm{Ca}: \mathrm{TiO}_{2}-\mathrm{B}$ and $\mathrm{TiO}_{2}-\mathrm{B}$ thin films has been carried out, as will be presented in detail below. Progressing parallel to the growth optimization, the atomic model of the new $\mathrm{Ca}: \mathrm{TiO}_{2}-\mathrm{B}$ phase has been gradually refined in density functional theory (DFT) calculations performed by Dr. Baihai Li, benefiting from the more and more clear STEM images obtained by Dr. Mike Katz from the high crystal quality thin films.

\subsection{Ca: $\mathrm{TiO}_{2}-\mathrm{B}$ thin film formation}

Although $\mathrm{TiO}_{2}-\mathrm{B}$ has the same stoichiometry as the other $\mathrm{TiO}_{2}$ polymorphs, it is far less stable in typical PLD growth conditions. Direct ablation from a pure $\mathrm{TiO}_{2}$ target onto a perovskite substrate usually results in the anatase or rutile phase, depending on the substrate surface structure and growth conditions. ${ }^{65,66}$ Figure 3.3 shows a (001) $\mathrm{TiO}_{2}$-anatase film grown on a (001) $\mathrm{SrTiO}_{3}$ substrate using a $\mathrm{TiO}_{2}$ target by PLD under $700{ }^{\circ} \mathrm{C}$ and $50 \mathrm{mTorr} \mathrm{O}_{2}$, well corresponding to its atomic structure in Figure 3.3(b). Except for an extended defect, which is possibly a grain boundary between two anatase grains that are rotated $90^{\circ}$ or $180^{\circ}$ from each other about the $c$-axis (equivalent directions due to symmetry), the pure anatase film is of high 
crystal quality.

Informed by the theoretical and experimental results described in Section 3.2, it was likely that Ca might help stabilize the bronze structure, forming a new phase. Hence, a target was constructed by mixing $90 \% \mathrm{TiO}_{2}$-anatase and $10 \% \mathrm{CaO}$ (mole percent) powders, which was then sintered and pressed into a dense pellet. The target was used to grow a thin film on a (001) $\mathrm{SrTiO}_{3}$ substrate under $700{ }^{\circ} \mathrm{C}$ and $50 \mathrm{mTorr}_{2}$ with a laser fluence of $3.4 \mathrm{~J} \mathrm{~cm}^{-2}$ and a repetition rate of $10 \mathrm{~Hz}$. The film, deposited in $20 \mathrm{~min}$, was discontinuous, consisting of small islands on the substrate surface, as shown in Figure 3.4. The grains were roughly epitaxial on the substrate, about up to $\sim 10 \mathrm{~nm}$ tall. Correlating with the atomic model as shown in Figure 1.1, it was clear that these were $\mathrm{Ca}: \mathrm{TiO}_{2}-\mathrm{B}$ grains, some of which were viewed along the [100] direction (Figure 3.4(b)), while others were viewed along the [010] direction (Figure 3.4(c-d)). Therefore, the film was approximately (001) oriented, although in many grains the $a-b$ planes were slightly tilted, possibly due to certain defect phase formation roughening the interface with the substrate.

Increasing the growth temperature significantly changed the morphology of the film. Grown at $800{ }^{\circ} \mathrm{C}$ with other conditions fixed using the same $10 \% \mathrm{CaO}$ target also in $20 \mathrm{~min}$, the film became continuous with a larger thickness of $\sim 26 \mathrm{~nm}$, as shown in Figure 3.5(a). As explained in Section 2.1.2, the higher temperature has apparently facilitated the grain growth and enhanced the deposition rate, thus increasing the volume of the film both laterally and vertically. The film exhibits a co-existence of the $\mathrm{Ca}: \mathrm{TiO}_{2}-\mathrm{B}$ and anatase phases, while different $\mathrm{Ca}: \mathrm{TiO}_{2}-\mathrm{B}$ grains are aligned in different orientations. These microstructure features are further discussed in the section as follows.

\subsection{Growth optimization of the $\mathrm{Ca}: \mathrm{TiO}_{2}-\mathrm{B}$ thin films}

\subsubsection{Target composition}

According to the theoretical calculations, ${ }^{62}$ the $\mathrm{Ca}: \mathrm{TiO}_{2}-\mathrm{B}$ phase in a $\mathrm{Ca}_{4} \mathrm{Ti}_{20} \mathrm{O}_{44}$ unit cell is a thermodynamically stable member of the $\mathrm{CaO}-\mathrm{TiO}_{2}$ pseudo-binary system, and its lattice constants were previously given in Section 1.2 . The $\mathrm{CaTi}_{5} \mathrm{O}_{11}$ stoichiometry corresponds to a 
Ti:Ca ratio of $5: 1$, or equivalently, $1 / 6$ (by mole) $\mathrm{CaO}$ in the powder mixture that was used to make the PLD target. Experimentally, we made targets containing $10 \%, 16.7 \%$ and $20 \% \mathrm{CaO}$ for comparison. The targets were installed in the Neocera PLD system with a chamber base pressure $<10^{-7}$ Torr, facing substrates at a distance of $6.35 \mathrm{~cm}$. The laser repetition rate was set at $10 \mathrm{~Hz}$ to ablate the targets.

$\mathrm{SrTiO}_{3}$ substrates are intrinsically fitting for growing $\mathrm{TiO}_{2}$ based structures on top, as a previous study illustrated that $\mathrm{SrTiO}_{3}(001)$ has a $\mathrm{TiO}_{2}$-rich surface. ${ }^{67}$ Further, good epitaxy of (001) $\mathrm{Ca}: \mathrm{TiO}_{2}-\mathrm{B}$ thin films on (001) $\mathrm{SrTiO}_{3}$ substrates is expected because its in-plane $a$ and $b$ cell parameters are nearly integer multiples of the lattice constant of cubic $\mathrm{SrTiO}_{3}(3.905 \AA)$, presenting a lattice mismatch of $\sim 3.25 \%$ (calculated for diagonal mismatch). Figure 3.6 compares the $\theta-2 \theta$ patterns of the thin films deposited from the targets of different compositions onto (001) $\mathrm{SrTiO}_{3}$ substrates at $800{ }^{\circ} \mathrm{C}$ in an oxygen ambient of 0.05 Torr, where the laser pulse energy was set to $200 \mathrm{~mJ}$ (a fluence of $\sim 3.4 \mathrm{~J} \mathrm{~cm}^{-2}$ ). With $10 \% \mathrm{CaO}$ in the target, multiple $00 \mathrm{l}$ peaks of $\mathrm{Ca}: \mathrm{TiO}_{2}-\mathrm{B}$ are seen at $2 \theta$ angles well corresponding to the theoretical calculations. The insufficiency of $\mathrm{Ca}$ caused some grains to crystallize in the anatase phase, the (001) plane of which also has a small lattice mismatch (-3.15\%) with $\mathrm{SrTiO}_{3}(001)$, evidenced by a prominent anatase 004 peak in XRD. Figure 3.5(b) shows a HAADF STEM image taken along the [010] direction of the $\mathrm{SrTiO}_{3}$. The film is mainly comprised of the $\mathrm{Ca}: \mathrm{TiO}_{2}-\mathrm{B}$ phase in its signature layered bronze structure, with the Ti-Ca interleaved layer turning the regular $\mathrm{TiO}_{2}-\mathrm{B}$ structure into a twinned zigzag pattern (see ref. 12 for atomic model). Since the cubic substrate has four equivalent directions on the surface, $\mathrm{Ca}: \mathrm{TiO}_{2}-\mathrm{B}$ grains can form in four different orientations with its [100] direction aligned along one of the $<100>$ directions on the (001) $\mathrm{SrTiO}_{3}$ substrate surface. As seen in Figure 3.5(b), the [010] direction of the large $\mathrm{Ca}: \mathrm{TiO}_{2}-\mathrm{B}$ grain on the top-left side is parallel to the [010] direction of the $\mathrm{SrTiO}_{3}$ substrate, while the small grain outlined in the top-right corner is viewed along the [100] direction, presenting a $90^{\circ}$ (or $270^{\circ}$ ) rotation about the [001] direction from the neighboring grain. This grain rotation effect is repeatedly observed throughout the film, as shown in Figure 3.5(c). Besides the $\mathrm{Ca}: \mathrm{TiO}_{2}-\mathrm{B}$ grains viewed along the 
[100] direction on the left and right of the image, one other grain in such orientation sits near the surface of the film, on top of an extended defect, while approximately maintaining its epitaxial relationship with the surrounding grains. The epitaxial relationships at the film-substrate interface are depicted in Figure 3.7, consistent with the observations in STEM. A few anatase grains growing along its $c$-axis were also observed (Figure 3.5). Images taken at lower magnifications indicate that the anatase phase occupies approximately $5-10 \%$ of the volume of the film, though it should be noted that most anatase grains are located near the interface with the substrate. Once the $\mathrm{Ca}: \mathrm{TiO}_{2}-\mathrm{B}$ phase is formed, it offers a good template for continuous upward stacking of the atom layers in the $a-b$ planes, and therefore the film surface is cleanly $\mathrm{Ca}: \mathrm{TiO}_{2}-\mathrm{B}$. As the $\mathrm{Ca}$ content is increased in the target, the anatase 004 peak is gradually suppressed relative to the $\mathrm{Ca}: \mathrm{TiO}_{2}-\mathrm{B}$ peaks. The target containing $20 \% \mathrm{CaO}$ resulted in the best films so far with strongest $00 l$ reflections from the bronze planes (bottom pattern in Figure 3.6), and only a trace amount of anatase. The STEM image in Figure 3.8(a) confirms that the film is $\sim 65 \mathrm{~nm}$ thick and is almost entirely of the $\mathrm{Ca}: \mathrm{TiO}_{2}-\mathrm{B}$ phase, with a smooth surface.

$\mathrm{Ca}$ is of vital importance in stabilizing the structure. Figure 3.8(b) shows the high magnification HAADF image of the film deposited using the $20 \% \mathrm{CaO}$ target. While the film is comprised entirely of the $\mathrm{Ca}: \mathrm{TiO}_{2}-\mathrm{B}$ phase, crystal defects can be identified stemming from stacking faults where the Ti-Ca interleaved layer is partially missing, creating a somewhat disordered boundary separating two grains, which is possibly caused by slight perturbation of material flow in the PLD plasma plume, or inhomogeneity in the target composition. In another case, instead of being partially missing, the Ti-Ca layer may be broken up and slightly shifted across two adjacent grains, forming an anti-phase boundary as an extended defect, as shown in Figure $3.8(\mathrm{c})$. The fact that the $20 \% \mathrm{CaO}$ target worked better than the stoichiometric $16.7 \%$ $\mathrm{CaO}$ target indicates that, in this specific thermodynamic environment during growth, $\mathrm{Ti}$ in the targets has a higher efficiency of transferring into the film than $\mathrm{Ca}$ does. Such observation helps explain the lower $\mathrm{Ca}: \mathrm{TiO}_{2}-\mathrm{B}$ purity in films grown from targets that have less Ca content, where the deficiency of $\mathrm{Ca}$ starves a few grains into forming $\mathrm{TiO}_{2}$-anatase. 


\subsubsection{Choice of substrates}

To explore the possibility of growing bronze films on other substrates, a few commonly used crystals have been tested, and their lattice structures are listed in Table $3.1 .^{66}$ The $\theta-2 \theta$ XRD patterns of the films deposited on various substrates from a $20 \% \mathrm{CaO}$ target with growth conditions fixed as above are compared in Figure 3.9. Among the five, only $\mathrm{LaAlO}_{3}$ led to the formation of the $\mathrm{Ca}: \mathrm{TiO}_{2}-\mathrm{B}$ phase, where anatase is also prominent, and no $\mathrm{Ca}: \mathrm{TiO}_{2}-\mathrm{B}$ formation can be identified on the rest. $\mathrm{CaTiO}_{3}$ phase was found on $\mathrm{Si}$ and $\mathrm{MgO}$, while $\mathrm{TiO}_{2}$-rutile could be identified on $\mathrm{YSZ}$ and $\mathrm{Al}_{2} \mathrm{O}_{3} \cdot{ }^{66,68}$ The resultant phases were mostly driven by their epitaxial relationship with the substrates. Table 3.2 summarizes the in-plane mismatch at the film-substrate interface, where it is clear that the structure which the films eventually crystallized in is determined by a combination of small lattice mismatch, similar crystal symmetry and target composition. There are a few unidentified peaks, sometimes quite broad, in each XRD pattern, which are likely associated with more complicated phases such as $\mathrm{Ti}_{4} \mathrm{O}_{7}$ (01-077-1392) or $\mathrm{CaTi}_{2} \mathrm{O}_{4}$ (04-010-1325) in less crystalline form, although these non-dominant phases were not observed in TEM (typical specimens have a viewing area of $\sim 1 \mu \mathrm{m}$ ).

\subsubsection{Growth temperature, laser energy and $\mathrm{O}_{2}$ partial pressure}

Understanding that (001) $\mathrm{SrTiO}_{3}$ is by far the most suitable substrate for the growth of $\mathrm{Ca}: \mathrm{TiO}_{2}-\mathrm{B}$, the effects of varying growth temperature, laser energy and $\mathrm{O}_{2}$ partial pressure were further investigated. In order to monitor the purity of the desired $\mathrm{Ca}: \mathrm{TiO}_{2}-\mathrm{B}$ phase relative to the major impurity, anatase, in this study, a target containing $10 \% \mathrm{CaO}$ was used so that the anatase 004 peak was usually manifest, as discussed above. If a $20 \% \mathrm{CaO}$ target was used, the anatase peak would generally be much weaker, making it hard to track against varying growth conditions. Figure 3.10 compares the XRD patterns of the films grown in the temperature range of $600-900{ }^{\circ} \mathrm{C}$ with other conditions fixed. The existence of anatase was observed in all samples, and its 004 peak was strongest at $700{ }^{\circ} \mathrm{C}$. At that same temperature, the $\mathrm{Ca}: \mathrm{TiO}_{2}-\mathrm{B}$ phase started 
to form showing a broad 006 peak, which became stronger and sharper with increasing temperature until the optimal result at $800{ }^{\circ} \mathrm{C}$, and then broadened and weakened again up to $900{ }^{\circ} \mathrm{C}$. On the other hand, the formation of the $\mathrm{Ca}: \mathrm{TiO}_{2}-\mathrm{B}$ phase was not very sensitive to the laser pulse energy, as samples of similar quality were obtained using laser energy around $200 \mathrm{~mJ}$ (a fluence of $\sim 3.4 \mathrm{~J} \mathrm{~cm}^{-2}$ ). However, if the laser pulses were overly powerful, the excess amount of $\mathrm{Ti}$ in the plasma plume would force part of the film to crystallize in cubic TiO phase rather than $\mathrm{TiO}_{2}$, as seen in Figure 3.11(a). The impact of $\mathrm{O}_{2}$ partial pressure on the thin film growth is shown in Figure 3.11(b). With no $\mathrm{O}_{2}$ backfilling, anatase formation was prohibited and a faint $\mathrm{Ca}: \mathrm{TiO}_{2}-\mathrm{B} 006$ peak was observed. The increasing pressure of $\mathrm{O}_{2}$ helped fill oxygen vacancies in the bronze structure, yielding the best result at 0.05 Torr, beyond which the material flow was scattered and ion path shortened with almost no film deposited at 0.1 Torr.

\section{5 $\mathrm{TiO}_{2}-\mathrm{B}$ thin film growth on a $\mathrm{Ca}: \mathrm{TiO}_{2}-\mathrm{B}$ template layer}

It was found through further investigations that $\mathrm{Ca}: \mathrm{TiO}_{2}-\mathrm{B}$ can serve as a template for growing regular $\mathrm{TiO}_{2}-\mathrm{B}$ on top due to the near-perfect lattice match between the two, where their mismatch in the $a-b$ plane is $-0.075 \%$ (Table 3.2 ). ${ }^{8}$ The crystal structure of $\mathrm{TiO}_{2}-\mathrm{B}$ epitaxy on $\mathrm{Ca}: \mathrm{TiO}_{2}-\mathrm{B}$ that has been optimized by VASP software ${ }^{10}$ is shown in Figure 3.12, where the two phases align their respective $a-b$ planes with each other, bonded by a layer of oxygen. As a result, high quality (001) $\mathrm{TiO}_{2}$-B thin films could be deposited on a (001) $\mathrm{Ca}: \mathrm{TiO}_{2}-\mathrm{B}$ template layer using a pure $\mathrm{TiO}_{2}$ target under the same growth conditions as for $\mathrm{Ca}: \mathrm{TiO}_{2}-\mathrm{B}$, in contrast to the (001) anatase film obtained from direct deposition of pure $\mathrm{TiO}_{2}$ on a (001) $\mathrm{SrTiO}_{3}$ substrate. ${ }^{69}$ The XRD pattern and a HAADF image of a $\mathrm{TiO}_{2}-\mathrm{B} / \mathrm{Ca}: \mathrm{TiO}_{2}-\mathrm{B} / \mathrm{SrTiO}_{3}$ heterostructure are illustrated in Figure 3.13(a) (top part) and Figure 3.13(b), corresponding well with the atomic model in Figure 3.12. The structure parameters of our $\mathrm{TiO}_{2}-\mathrm{B}$ films are in good agreement with values in the literature. ${ }^{8,70}$ The theoretical densities of $\mathrm{Ca}: \mathrm{TiO}_{2}-\mathrm{B}$ and $\mathrm{TiO}_{2}-\mathrm{B}$ are determined to be $3.637 \mathrm{~g} \mathrm{~cm}^{-3}$ and $3.616 \mathrm{~g} \mathrm{~cm}^{-3}$, respectively, approximately $7 \%$ lower than that of anatase.

The templating effect in such a dual layer film is obvious. Depending on the terminating 
surface in its zigzag structure of the $\mathrm{Ca}: \mathrm{TiO}_{2}-\mathrm{B}$ layer, the $\mathrm{TiO}_{2}-\mathrm{B}$ phase could be growing along two opposite directions in the upward stacking of the atom layers in the $a-b$ planes, as seen in Figure 3.13(c). Originating from the defect (indicated by the arrow) of a partially missing Ti-Ca interleaved layer, the $\mathrm{Ca}: \mathrm{TiO}_{2}-\mathrm{B}$ phase terminates with different surfaces that are two Ti layers apart, resulting in an anti-phase boundary above it where two $\mathrm{TiO}_{2}-\mathrm{B}$ grains collide. A number of variations to such defects also exist, changing the terminating surface of the $\mathrm{Ca}: \mathrm{TiO}_{2}-\mathrm{B}$ template layer, so the abutting $\mathrm{TiO}_{2}$-B grains on top may stack away from each other, as shown in Figure 3.14(a), or may run upwards in the same direction when two Ti-Ca layers are partially missing, as shown in Figure 3.14(b). More examples and detailed analysis on these crystal defects and their forming mechanism can be found in Ref. 71.

Crystal quality of the $\mathrm{TiO}_{2}$ - $\mathrm{B}$ thin films was then examined by monitoring the full width at half maximum (FWHM) of the $\mathrm{TiO}_{2}-\mathrm{B} 002$ (strongest) peak. The average grain sizes can be calculated using the Scherrer equation, ${ }^{72}$

$$
\tau=\frac{K \lambda}{\beta \cos \theta}
$$

where $\tau$ is the mean size of the ordered crystalline domains (may be smaller or equal to the grain size), $K$ is a dimensionless shape factor with a value close to unity and often taken as $0.9, \lambda$ is the $\mathrm{x}$-ray wavelength $(\lambda=1.54 \AA$ for $\mathrm{Cu}-\mathrm{K} \alpha$ radiation), $\beta$ is the FWHM value after subtracting the instrumental line broadening, and $\theta$ is the Bragg angle. Both the FWHM values and the average grain sizes are plotted as functions of the $\mathrm{TiO}_{2}-\mathrm{B}$ film thickness in Figure 3.15(a). Below $\sim 40 \mathrm{~nm}$, the grain size increases proportionally with the film thickness, resulting in a decreasing FWHM. As the thickness further increases, the grain size is often limited in the lateral dimension by crystal defects such as the anti-phase boundary in Figure 3.13(c), and shows a saturation behavior at $\sim 50 \mathrm{~nm}$, corresponding to a FWHM of $\sim 0.162^{\circ}$. The dependence of $\mathrm{TiO}_{2}-\mathrm{B} 002$ FWHM on the thickness of the underlying $\mathrm{Ca}: \mathrm{TiO}_{2}-\mathrm{B}$ template layer, however, is not significant. Except for the relatively large value associated with the smallest $\mathrm{TiO}_{2}-\mathrm{B}$ thickness, the $\mathrm{FWHM}$ is almost independent on the template thickness over a wide range, from 10 to $140 \mathrm{~nm}$, as seen in Figure 3.15(b). 
These results suggest that the templating effect can be transferred to other systems for $\mathrm{TiO}_{2}-\mathrm{B}$ applications, as long as a well-defined $\mathrm{Ca}: \mathrm{TiO}_{2}-\mathrm{B}(001)$ surface is formed. For instance, a conductive substrate is convenient for current collection when investigating the electrochemical properties of $\mathrm{TiO}_{2}-\mathrm{B}$ as a thin film anode in lithium-ion batteries. ${ }^{12}$ Using a 0.15 at.\% $\mathrm{Nb}$ doped $\mathrm{SrTiO}_{3}(001)$ substrate (resistivity $\sim 0.08 \Omega \mathrm{cm}$ ), a high quality dual layer film was also achieved (Figure 3.13(a)). Similar results were acquired from another sample grown on (001) LSAT, where it was demonstrated that the thicknesses of the two phases can be adjusted by controlling respective growth times: deposition time for $\mathrm{TiO}_{2}-\mathrm{B}$ was doubled ( $2 \mathrm{hr}$ to $4 \mathrm{hr}$ ) while it was decreased to $1 / 6$ for $\mathrm{Ca}: \mathrm{TiO}_{2}-\mathrm{B}$ ( $2 \mathrm{hr}$ to $20 \mathrm{~min}$ ), producing relatively stronger $\mathrm{TiO}_{2}-\mathrm{B}$ peaks in XRD (Figure 3.13(a)). A substantial amount of anatase existed in the $\mathrm{Ca}: \mathrm{TiO}_{2}-\mathrm{B}$ films grown on LSAT, likely caused by the fact that anatase has a smaller in-plane mismatch with LSAT than with $\mathrm{SrTiO}_{3}$ (Table 3.2). Similarly, since $\mathrm{Ca}: \mathrm{TiO}_{2}-\mathrm{B}$ proves to have a good epitaxy on (001) $\mathrm{SrTiO}_{3}$, the $\mathrm{TiO}_{2}-\mathrm{B} / \mathrm{Ca}: \mathrm{TiO}_{2}-\mathrm{B}$ dual layer structure may be grown on many other substrates where a (001) $\mathrm{SrTiO}_{3}$ buffer layer can be fabricated. As an example, Figure 3.16 show the $\theta-2 \theta$ XRD pattern of a (001) $\mathrm{TiO}_{2}-\mathrm{B} / \mathrm{Ca}: \mathrm{TiO}_{2}-\mathrm{B}$ dual layer film grown on a $\mathrm{SrTiO}_{3}$ (20 unit cells) buffered (100) Si substrate. The quality of the bronze film is essentially limited by the quality of the $\mathrm{SrTiO}_{3}$ buffer, whereas in this experiment, the buffer layer was not a perfect (001) film, as $\mathrm{SrTiO}_{3}$ (111) peak was also observed. The ability to grow bronze films on Si suggests great potential to integrate the properties of these new materials with existing infrastructure of the current semiconductor industry. Scientifically, we have used this sample to investigate the Raman properties of the bronze films, because in our test environment, it was much easier to isolate the substrate signal from that of the films which were grown on Si rather than on $\mathrm{SrTiO}_{3} .{ }^{50,73}$ Owing to the plate-by-plate growth mechanism of these structures, the $\mathrm{Ca}: \mathrm{TiO}_{2}-\mathrm{B}$ film usually has a rather smooth surface with a $1.77 \mathrm{~nm}$ root-mean-square (rms) roughness, which translates well to the $\mathrm{TiO}_{2}-\mathrm{B}$ layer above it with an rms surface roughness of $1.76 \mathrm{~nm}$, as shown by the atomic force microscopy (AFM) images in Figure 3.17(a-b), where the hills and valleys in the morphology are possibly associated with crystal defects that are extending to the 
surface (e.g. Figure 3.5 and Figure 3.8).

\subsection{Summary and conclusions}

In summary, high quality $\mathrm{TiO}_{2}-\mathrm{B}$ and $\mathrm{Ca}: \mathrm{TiO}_{2}-\mathrm{B}$ thin films with well-controlled lattice orientation and smooth surface can be fabricated on various substrates through a completely waterless process. Building on guidelines established here, multilayer devices that utilize both the bulk and the surface of these attractive materials may prove promising in a wider range of applications. Specifically, the following conclusions have been reached:

(1) High quality single crystalline (001) $\mathrm{Ca}: \mathrm{TiO}_{2}-\mathrm{B}$ thin films with a smooth surface can be deposited on (001) $\mathrm{SrTiO}_{3}$ substrates using a $(80 \mathrm{~mol} \% \mathrm{TiO} 2+20 \mathrm{~mol} \% \mathrm{CaO})$ target,

under the optimal growth conditions of $800{ }^{\circ} \mathrm{C}, 50$ mTorr $\mathrm{O}_{2}, 3.4 \mathrm{~J} \mathrm{~cm}^{-2}$ laser fluence and $10 \mathrm{~Hz}$ repetition rate.

(2) Relatively high quality $\mathrm{Ca}: \mathrm{TiO}_{2}-\mathrm{B}$ thin films may also be grown on (001) $\mathrm{LaAlO}_{3}$ and (001) LSAT, where anatase is a major defect phase usually locating near the interface with the substrate, due to the small lattice mismatch.

(3) High quality single crystalline (001) $\mathrm{TiO}_{2}-\mathrm{B}$ thin films with a smooth surface can be deposited on a (001) $\mathrm{Ca}: \mathrm{TiO}_{2}-\mathrm{B}$ template layer using a pure $\mathrm{TiO}_{2}$ target, under the same conditions as those used to grow $\mathrm{Ca}: \mathrm{TiO}_{2}-\mathrm{B}$. The crystal quality of the $\mathrm{TiO}_{2}-\mathrm{B}$ films is largely independent on the template layer thickness. However, the grain size is often limited laterally by crystal defects.

(4) The stacking direction of the $\mathrm{TiO}_{2}-\mathrm{B}$ phase is determined by the terminating surface of the $\mathrm{Ca}: \mathrm{TiO}_{2}-\mathrm{B}$ template layer, resulting in various types of grain boundaries.

(5) Anywhere a (001) $\mathrm{SrTiO}_{3}$ surface exists, a (001) $\mathrm{Ca}: \mathrm{TiO}_{2}-\mathrm{B}$ thin film can grow on it; anywhere a (001) $\mathrm{Ca}: \mathrm{TiO}_{2}-\mathrm{B}$ surface is formed, a (001) $\mathrm{TiO}_{2}-\mathrm{B}$ thin film can grow on top. Therefore, the two bronze phases can essentially be synthesized on a wide variety of substrates, including $\mathrm{Si}$, for device applications. 
Table 3.1 Lattice structures of the phases and substrates involved in this study.

\begin{tabular}{llc}
\hline Phase & Crystal system & Lattice parameters \\
\hline $\mathrm{Ca}: \mathrm{TiO}_{2}-\mathrm{B}$ & orthorhombic & $a=12.1702 \AA, b=3.8013 \AA, c=17.9841 \AA$ \\
$\mathrm{TiO}_{2}-\mathrm{B}$ & monoclinic & $a=12.1787 \AA, b=3.7412 \AA, c=6.5249 \AA, \beta=107.054^{\circ}$ \\
$\mathrm{TiO}_{2}$-anatase & tetragonal & $a=b=3.7820 \AA, c=9.5150 \AA$ \\
$\mathrm{TiO}_{2}$-rutile & tetragonal & $a=b=4.5900 \AA, c=2.9600 \AA$ \\
$\mathrm{CaTiO}_{3}$ & pseudocubic & $a=3.8917 \AA$ \\
\hline $\mathrm{SrTiO}_{3}$ & cubic & $a=3.9051 \AA$ \\
$\mathrm{LSAT}^{a}$ & cubic & $a=3.8680 \AA$ \\
$\mathrm{LaAlO}_{3}$ & pseudocubic & $a=3.7913 \AA$ \\
$\mathrm{Si}$ & diamond cubic & $a=5.4305 \AA$ \\
$\mathrm{YSZ}^{b}$ & cubic & $a=5.1420 \AA$ \\
$\mathrm{MgO}$ & cubic & $a=4.2130 \AA$ \\
$\mathrm{Al}_{2} \mathrm{O}_{3}$ & hexagonal & $a=4.7580 \AA, c=12.9910 \AA$ \\
\hline
\end{tabular}

${ }^{a}\left(\mathrm{LaAlO}_{3}\right)_{0.3}\left(\mathrm{Sr}_{2} \mathrm{AlTaO}_{6}\right)_{0.7},{ }^{b}$ yttria-stabilized zirconia 
Table 3.2 In-plane mismatch (calculated on a diagonal basis) between difference phases and the substrate surfaces. The preferred phase eventually adopted by the film deposited from a $(80 \%$ $\mathrm{TiO}_{2}+20 \% \mathrm{CaO}$ ) target on each specific substrate is set in bold.

\begin{tabular}{|c|c|c|c|c|c|}
\hline Substrate surface & $\begin{array}{c}\mathrm{Ca}: \mathrm{TiO}_{2}-\mathrm{B} \\
(001)\end{array}$ & $\begin{array}{c}\mathrm{TiO}_{2} \text {-anatase } \\
(001)\end{array}$ & $\begin{array}{c}\mathrm{TiO}_{2} \text {-rutile } \\
(001)\end{array}$ & $\begin{array}{c}\mathrm{CaTiO}_{3} \\
(001)\end{array}$ & $\begin{array}{c}\mathrm{TiO}_{2}-\mathrm{B} \\
(001)\end{array}$ \\
\hline $\mathrm{SrTiO}_{3}(001)$ & $3.25 \%$ & $-3.15 \%$ & $17.5 \%$ & $-0.343 \%$ & $3.17 \%^{a}$ \\
\hline LSAT (001) & $4.24 \%$ & $-2.22 \%$ & $18.7 \%$ & $0.613 \%$ & $4.16 \%^{a}$ \\
\hline $\mathrm{LaAlO}_{3}(001)$ & $6.35 \%$ & $-0.245 \%$ & $21.1 \%$ & $2.65 \%$ & $6.27 \%^{a}$ \\
\hline $\mathrm{Si}(100)$ & $8.58 \%$ & $4.47 \%$ & $-15.5 \%$ & $7.50 \%$ & $7.82 \%^{a}$ \\
\hline YSZ (100) & $14.7 \%$ & $10.3 \%$ & $-10.7 \%$ & $13.5 \%$ & $13.9 \%{ }^{a}$ \\
\hline $\mathrm{MgO}(100)$ & $-4.30 \%$ & $-10.2 \%$ & $8.95 \%$ & $-7.63 \%$ & $-4.37 \%^{a}$ \\
\hline$\alpha-\mathrm{Al}_{2} \mathrm{O}_{3}(0001)$ & $-22.6 \%$ & $-11.1 \%$ & $7.95 \%$ & $-8.48 \%$ & $-22.6 \%^{a}$ \\
\hline $\mathrm{Ca}: \mathrm{TiO}_{2}-\mathrm{B}(001)$ & 0 & $-6.20 \%$ & $13.8 \%$ & $-3.48 \%$ & $-0.075 \%$ \\
\hline
\end{tabular}

${ }^{a}$ For comparison only. $\mathrm{TiO}_{2}-\mathrm{B}$ does not grow directly on these substrates in this study. 

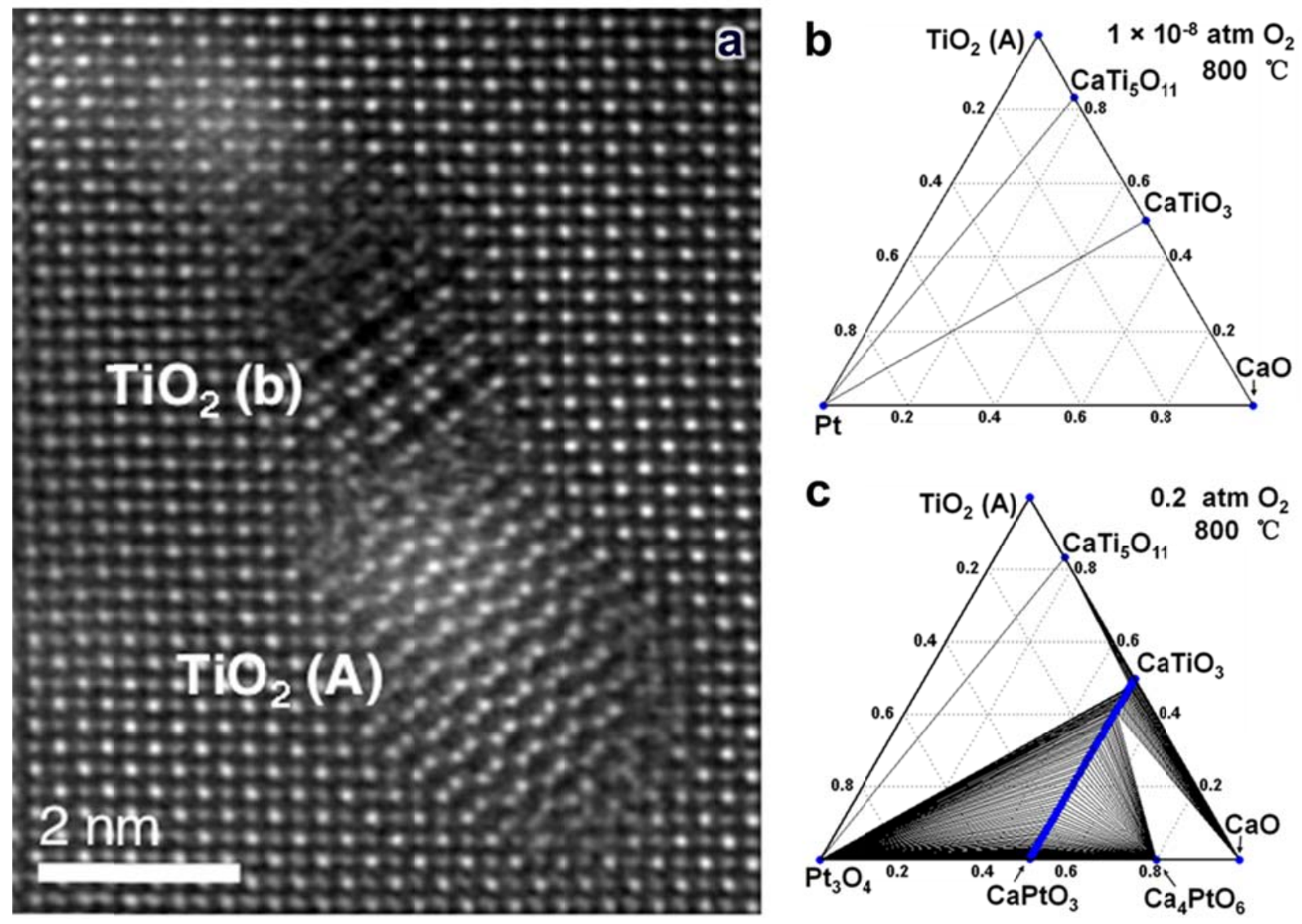

Figure 3.1 (a) $\mathrm{TiO}_{2}$-anatase and $\mathrm{TiO}_{2}-\mathrm{B}$ inclusions in the $\mathrm{CaTi}_{0.95} \mathrm{Pt}_{0.05} \mathrm{O}_{3}$ thin film after reduction treatment. (b-c) Calculated pseudo-ternary phase diagrams for the $\mathrm{CaO}_{\mathrm{x}}-\mathrm{TiO}_{\mathrm{y}}-\mathrm{PtO}_{z}$ system. [Figures reproduced from Ref. 62] 

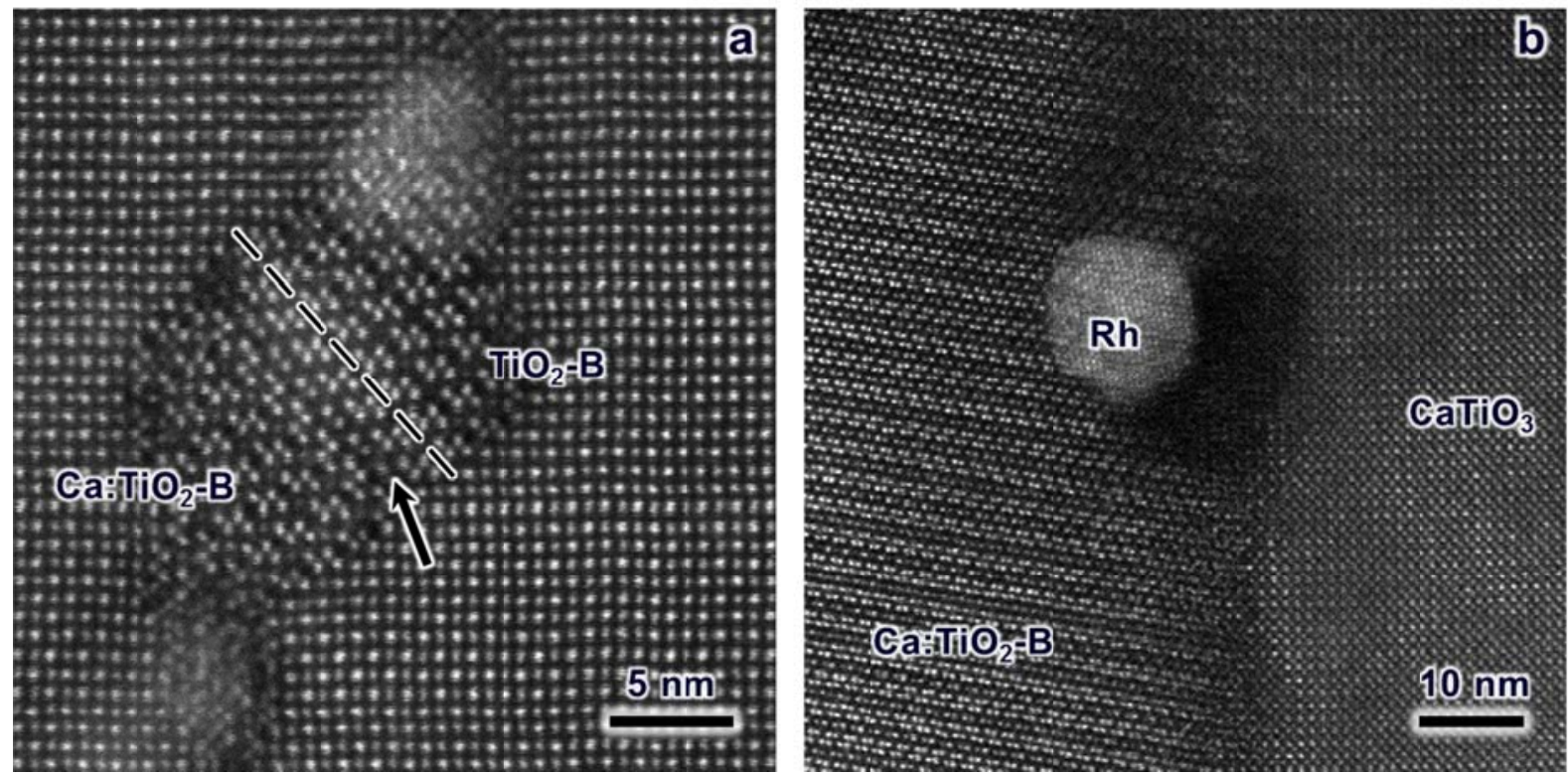

Figure 3.2 (a) $\mathrm{Ca}: \mathrm{TiO}_{2}-\mathrm{B}$ phase inclusion in the $\mathrm{CaTi}_{0.95} \mathrm{Pt}_{0.05} \mathrm{O}_{3}$ thin film after reduction treatment. The arrow marks a Ti-Ca layer in the $\mathrm{Ca}: \mathrm{TiO}_{2}-\mathrm{B}$ structure. The dashed line marks the interface between $\mathrm{Ca}: \mathrm{TiO}_{2}-\mathrm{B}$ and simple $\mathrm{TiO}_{2}-\mathrm{B}$. (b) A large $\mathrm{Ca}: \mathrm{TiO}_{2}-\mathrm{B}$ grain observed in a $\mathrm{CaTi}_{0.95} \mathrm{Rh}_{0.05} \mathrm{O}_{3}$ thin film after reduction treatment. [TEM image credit: Michael B. Katz] 

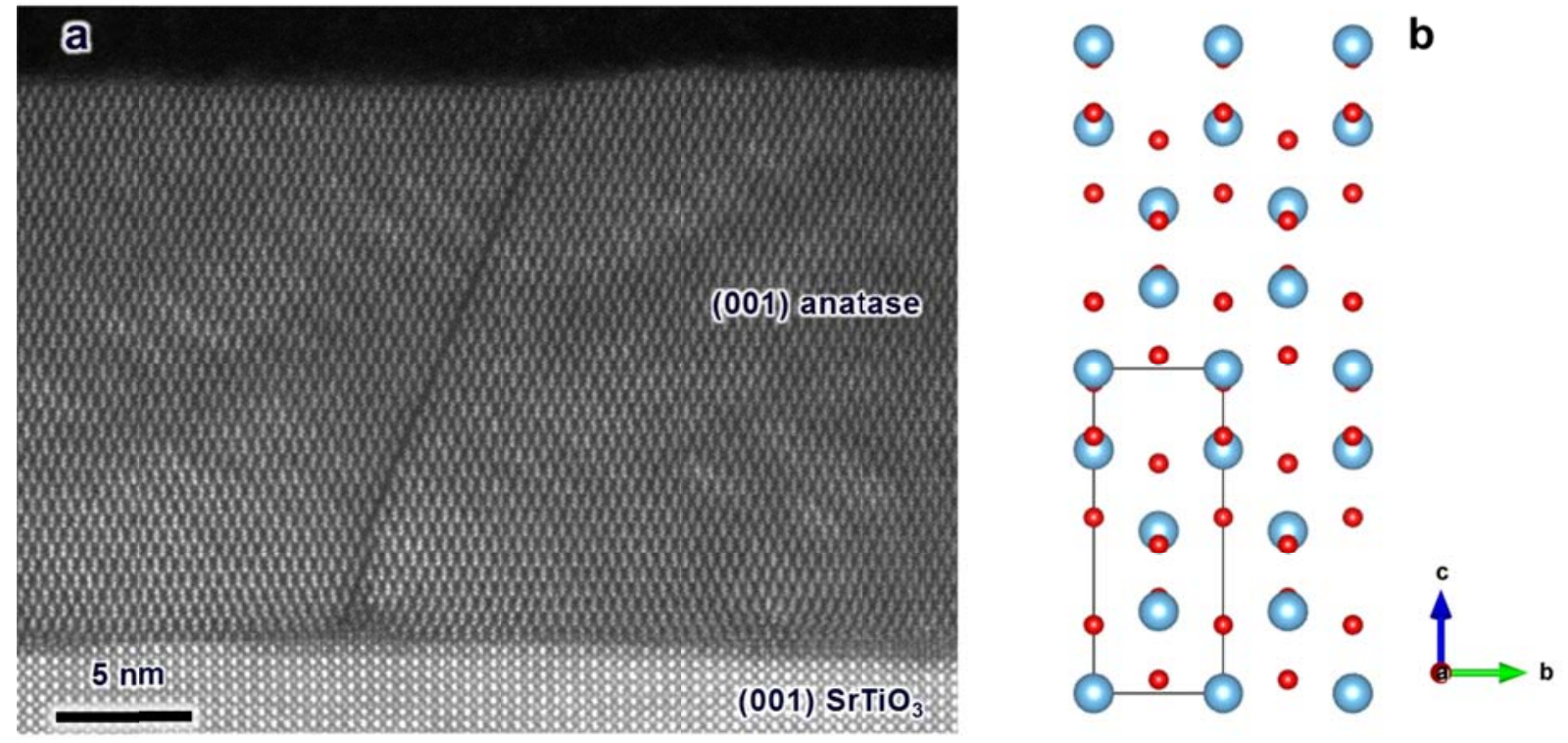

Figure 3.3 (a) HAADF STEM image of a (001) anatase film grown on a (001) $\mathrm{SrTiO}_{3}$ substrate by PLD. (b) The crystal structure of anatase projected along its [100] direction. [TEM image credit: Michael B. Katz] 

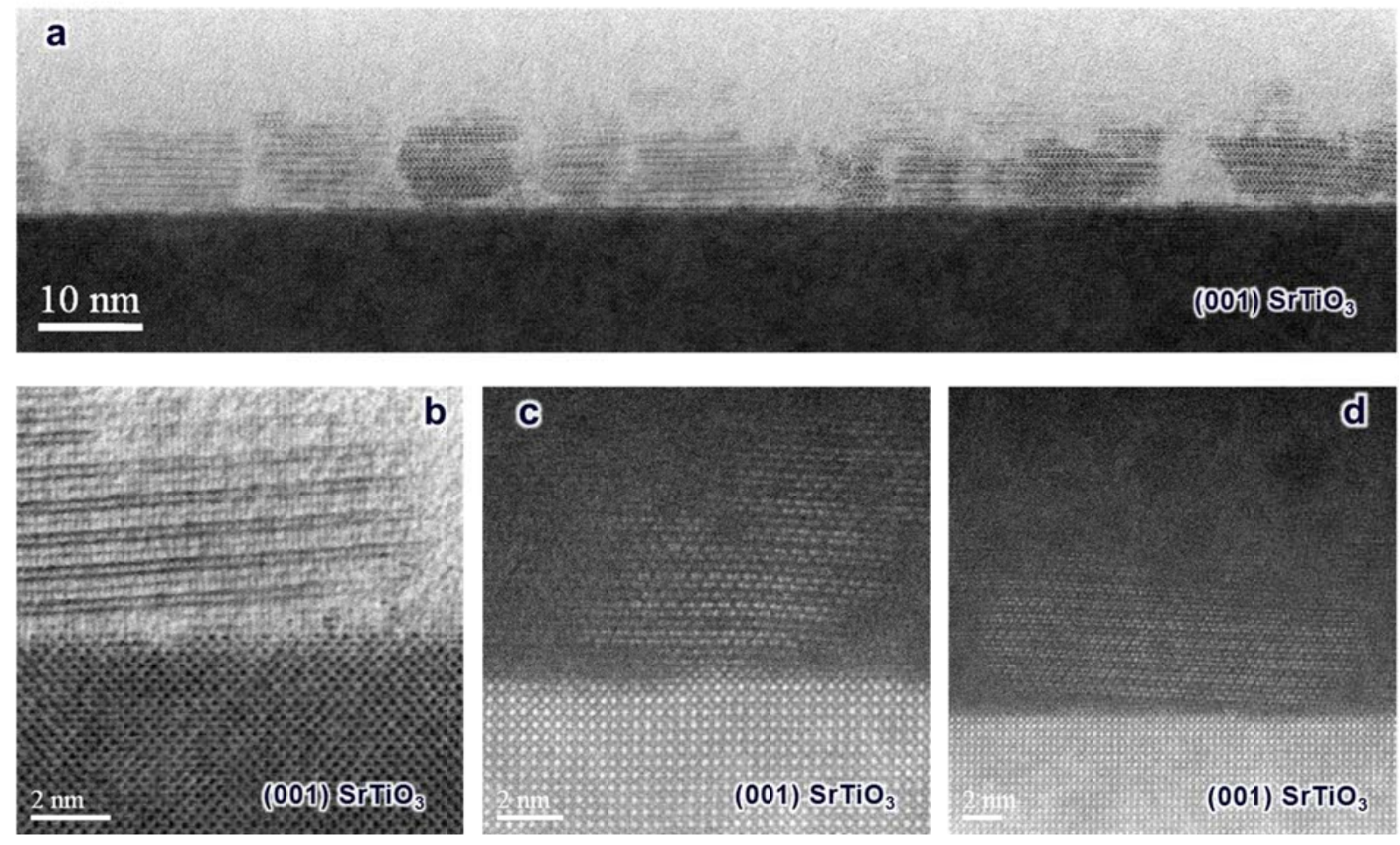

Figure 3.4 STEM images of a thin film deposited on (001) $\mathrm{SrTiO}_{3}$ using a $\left(\mathrm{TiO}_{2} 90 \%+\mathrm{CaO}\right.$ $10 \%$ ) target, showing a few grains in the $\mathrm{Ca}: \mathrm{TiO}_{2}-\mathrm{B}$ structure. (a-b) Bright field images. (c-d) Dark field images. [TEM image credit: Michael B. Katz] 


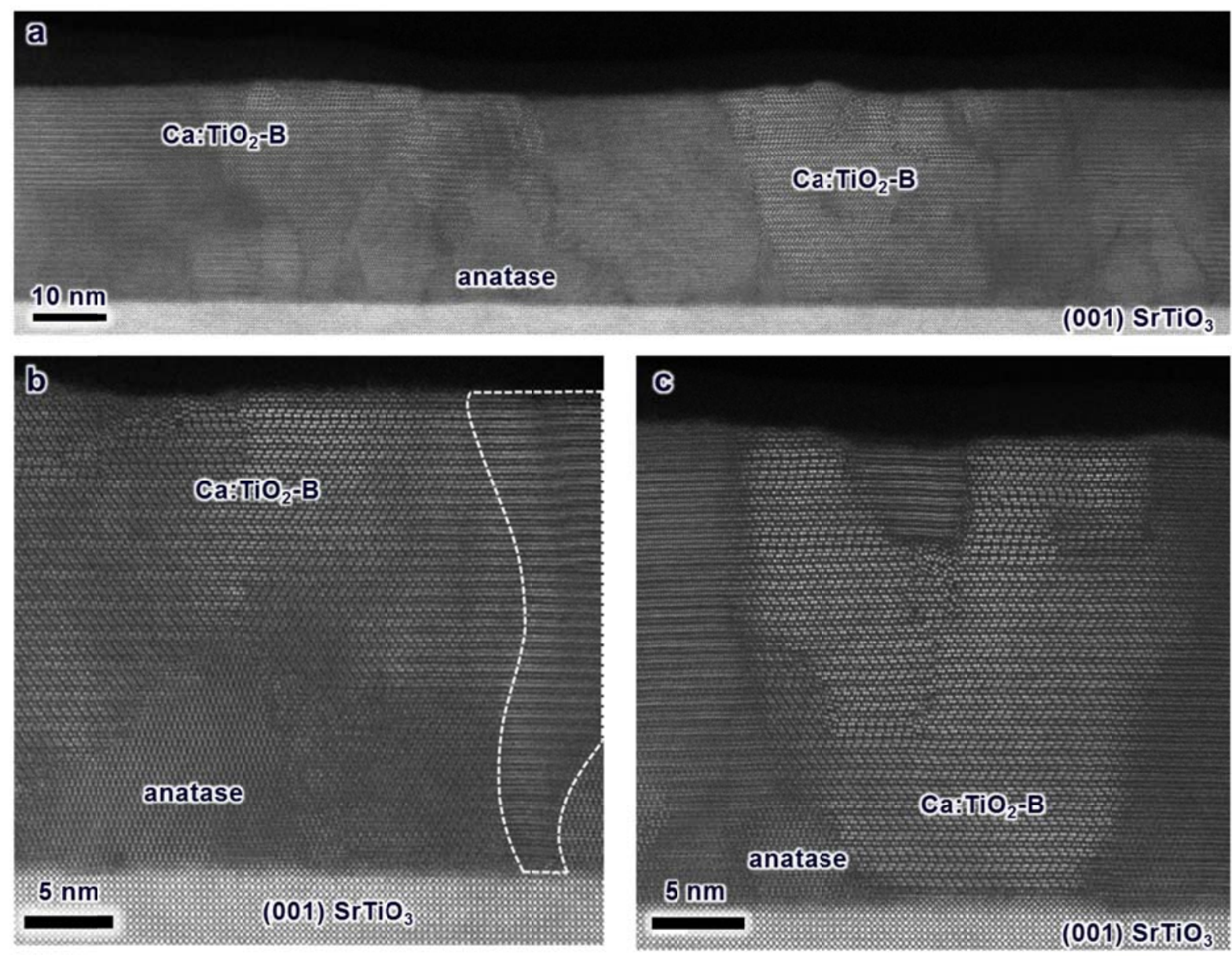

Figure 3.5 HAADF STEM images of a film deposited from a $\left(\mathrm{TiO}_{2} 90 \%+\mathrm{CaO} 10 \%\right)$ target showing the co-existence of $\mathrm{Ca}: \mathrm{TiO}_{2}-\mathrm{B}$ and anatase. (a) A low magnification image of the film. (b) A higher magnification image showing a $\mathrm{Ca}: \mathrm{TiO}_{2}-\mathrm{B}$ grain in a different orientation from its neighboring grain, which is encompassed by the dashed line. (c) More instances showing the grain rotation about the $c$-axis of $\mathrm{Ca}: \mathrm{TiO}_{2}$-B. [TEM image credit: Michael B. Katz] 


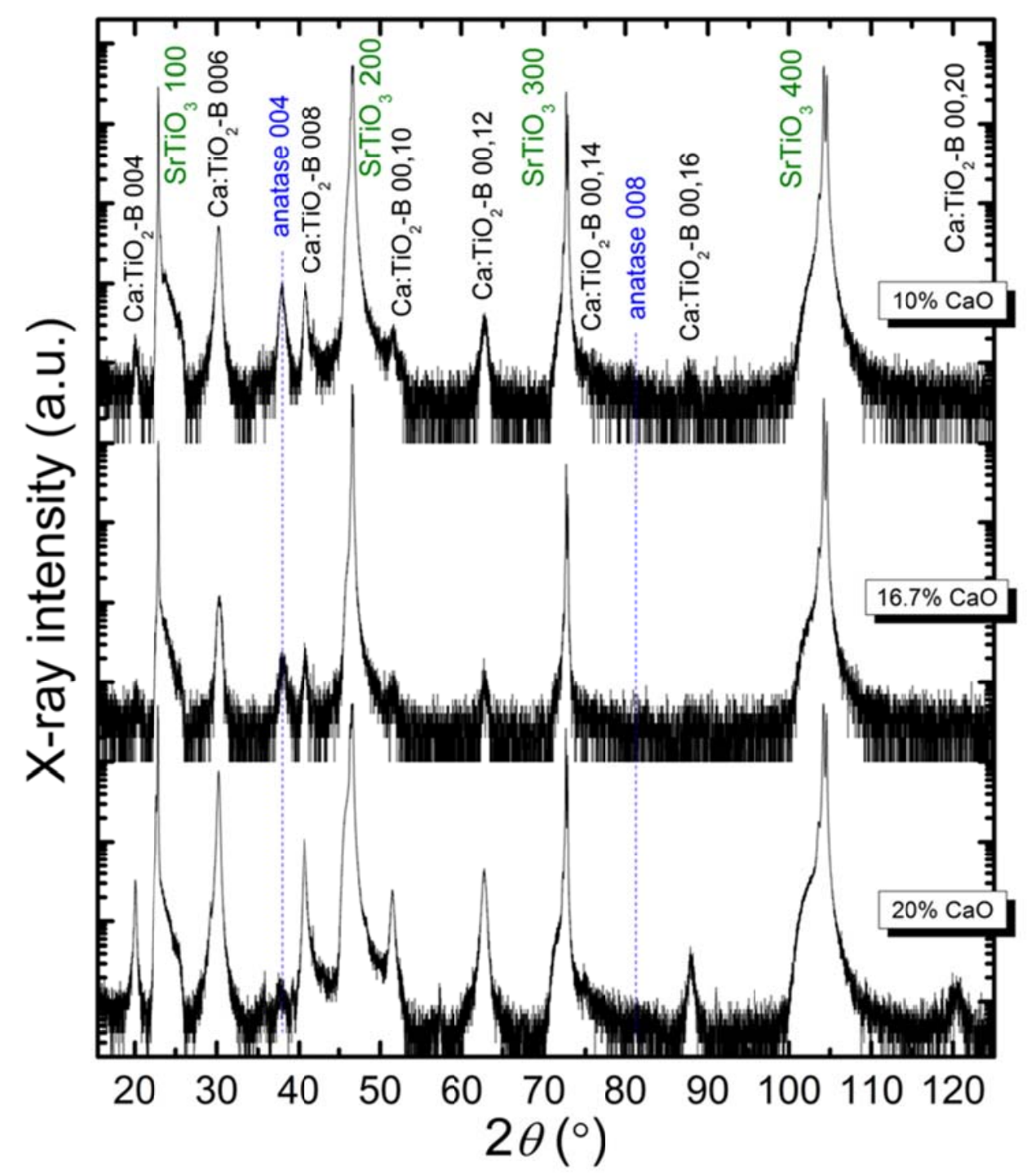

Figure 3.6 XRD patterns of thin films deposited from targets of different compositions. The 20\% $\mathrm{CaO}\left(80 \% \mathrm{TiO}_{2}\right.$, by mole) target resulted in a $(001) \mathrm{Ca}: \mathrm{TiO}_{2}-\mathrm{B}$ film with highest crystalline quality and only a trace amount of anatase. Vertical dashed lines mark the peak positions of the anatase phase. 


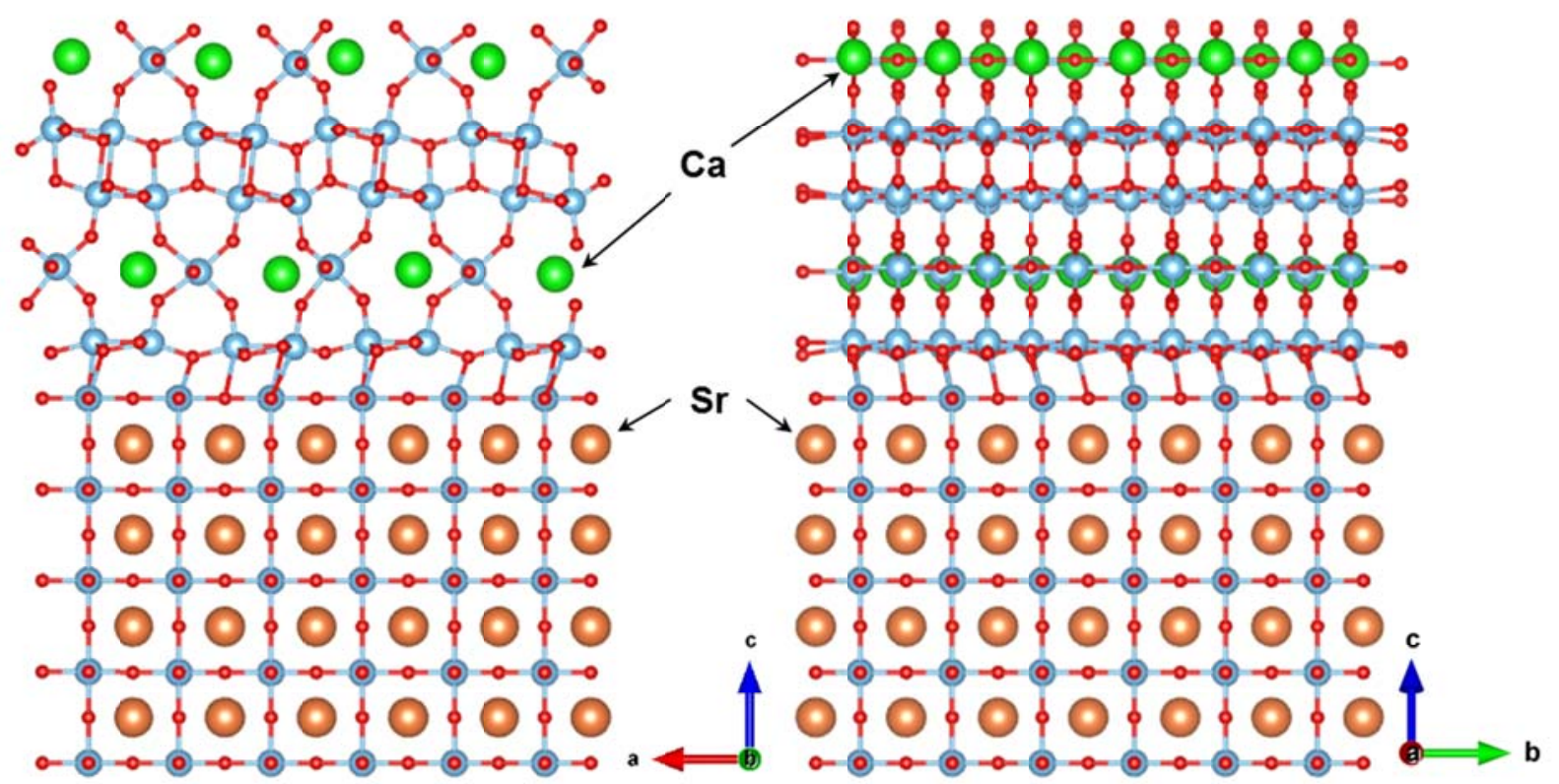

Figure 3.7 Crystal structure projected along [010] (left) and [100] (right) directions, showing the interfacial atomic structure and epitaxial relationship between $\mathrm{Ca}: \mathrm{TiO}_{2}-\mathrm{B}(001)$ and $\mathrm{SrTiO}_{3}(001)$ planes. 


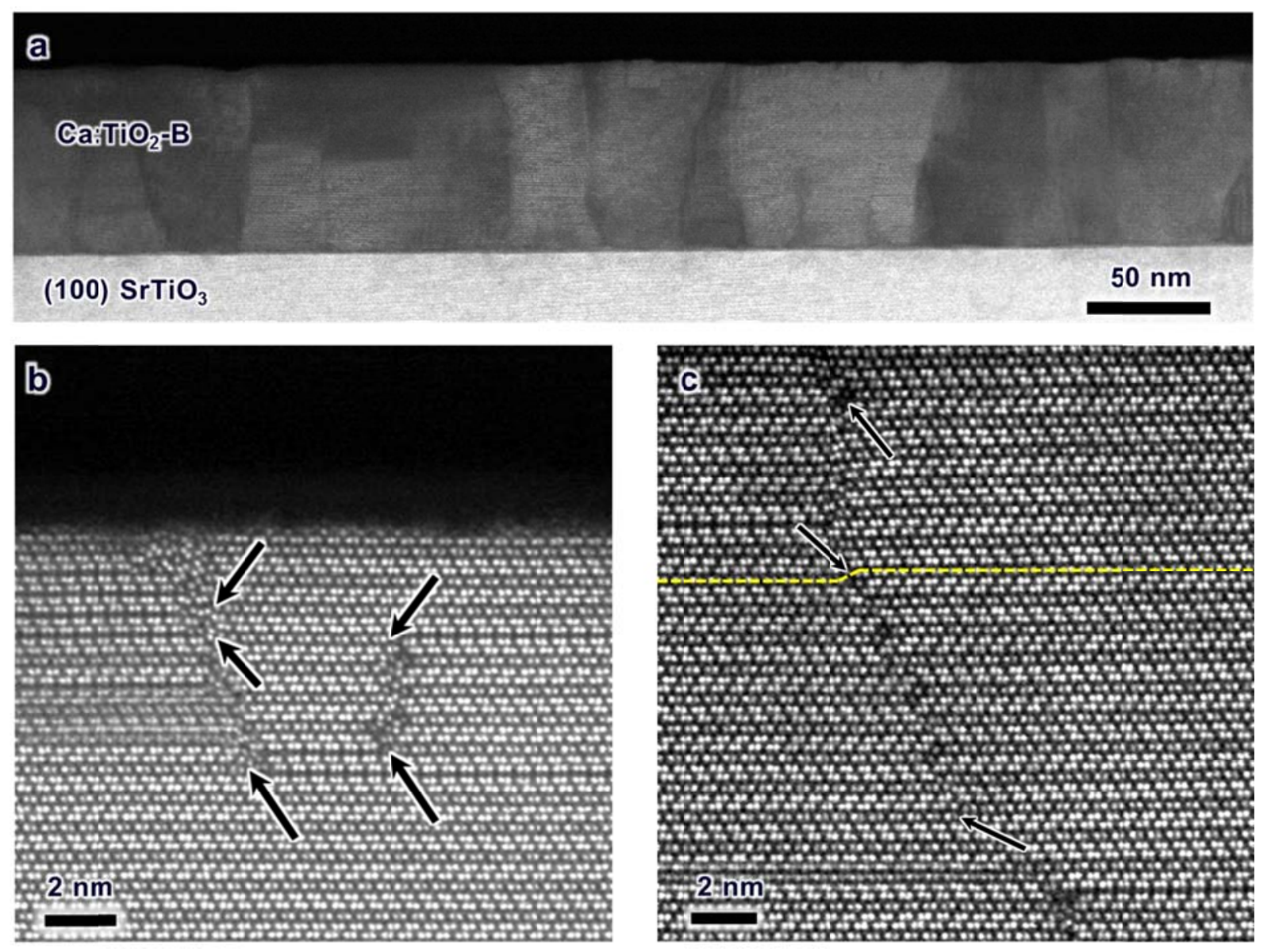

Figure 3.8 HAADF STEM images of a (001) Ca:TiO ${ }_{2}-\mathrm{B}$ film deposited from a $\left(\mathrm{TiO}_{2} 80 \%+\right.$ $\mathrm{CaO} 20 \%$ ) target on a (001) $\mathrm{SrTiO}_{3}$ substrate. (a) A low magnification image of the film with a smooth surface. (b) High magnification images showing the microstructure of the film. The arrows mark the stacking faults. (c) An anti-phase boundary caused by the shift of the Ti-Ca layer in two adjacent grains, marked by the arrows. The yellow dashed line depicts a discontinuous Ti-Ca layer with a jump at the anti-phase boundary. [TEM image credit: Michael B. Katz] 


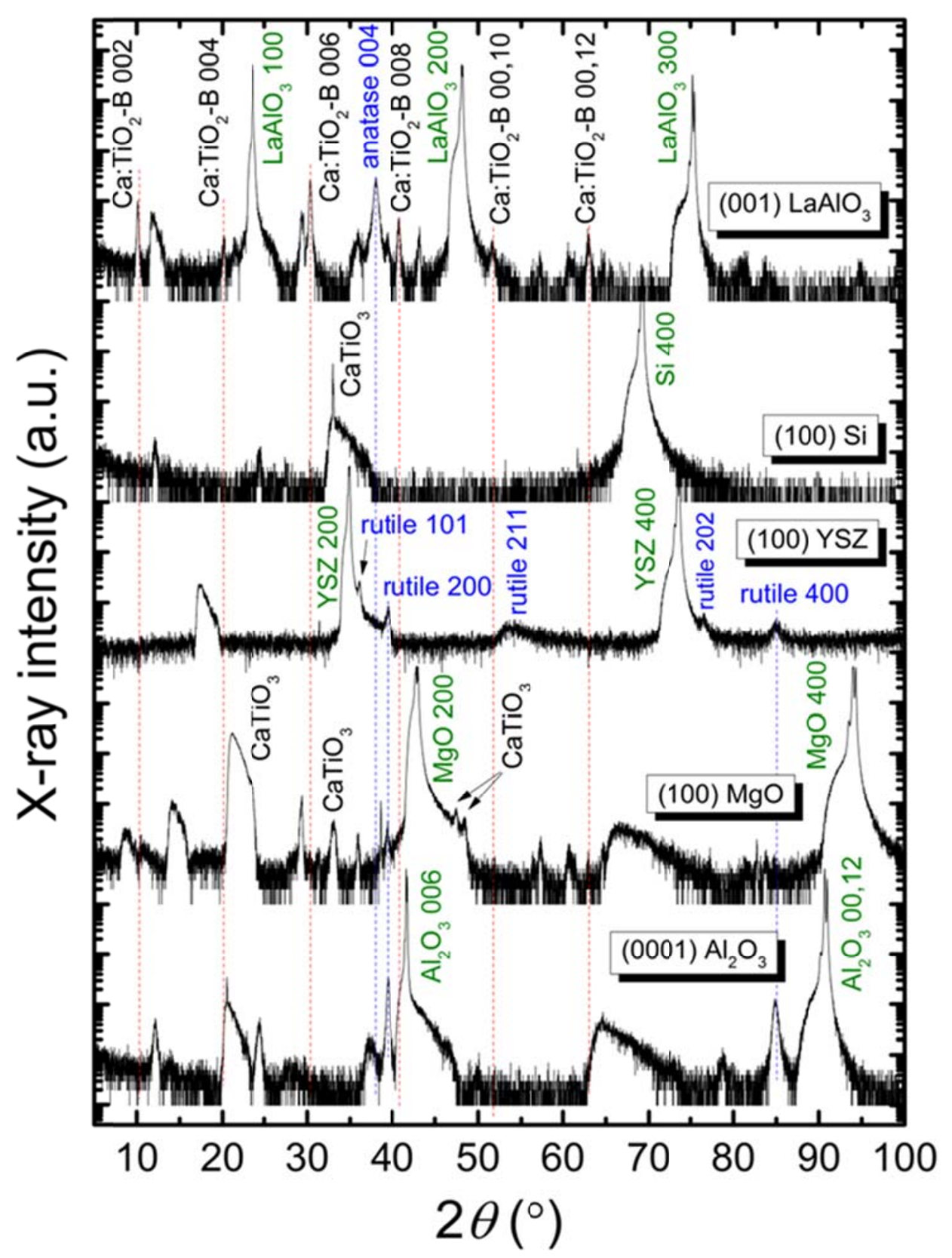

Figure 3.9 XRD patterns of the films deposited from a $20 \% \mathrm{CaO}$ target on 5 different substrates. Growth conditions were the same as those used to grow the films in Figure 3.6. Substrate peaks are labeled in green, and anatase/rutile peaks in blue. Vertical dashed lines are drawn to mark peak positions for comparison. 


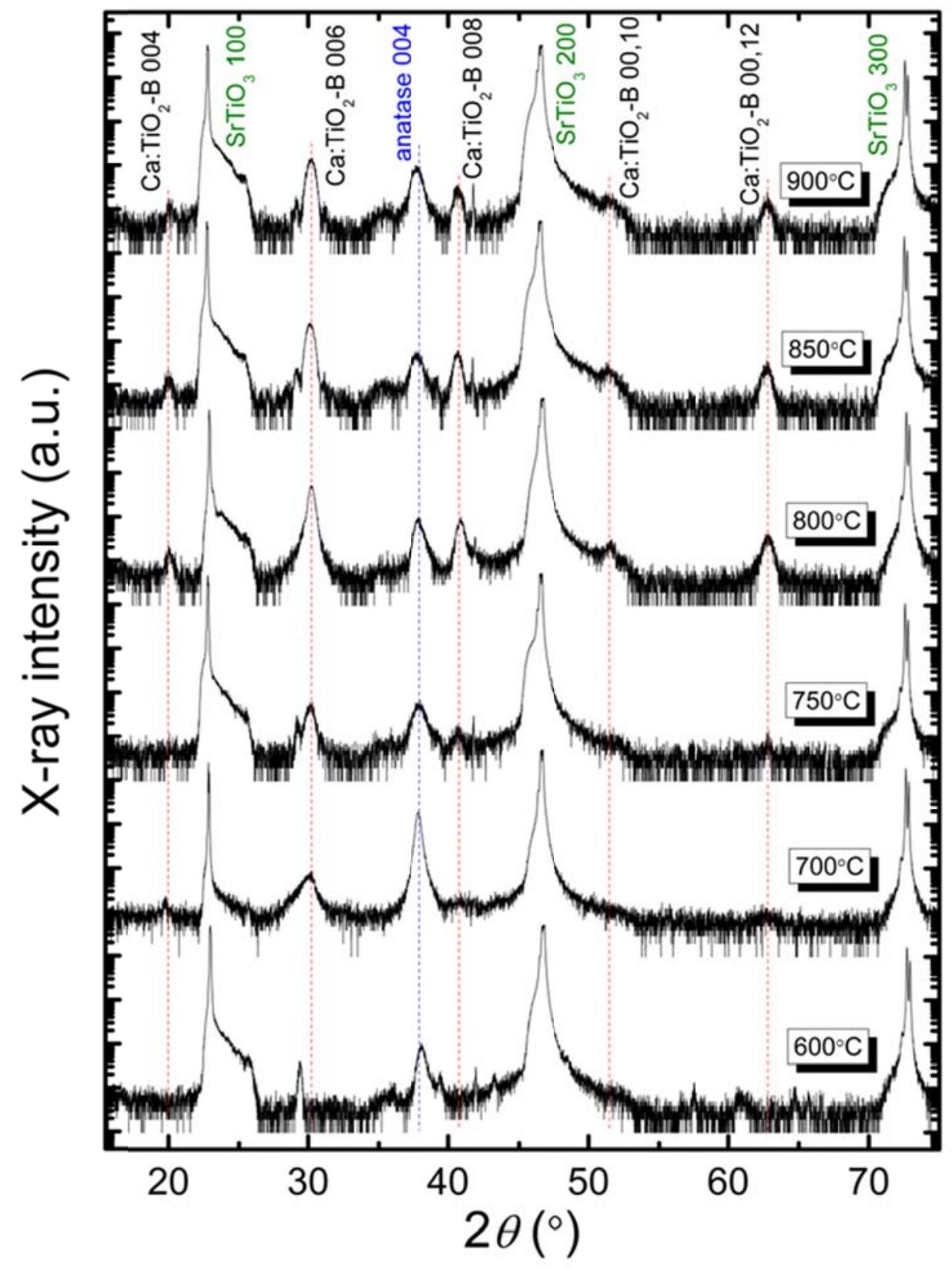

Figure 3.10 XRD patterns of the films deposited from a $10 \% \mathrm{CaO}$ target on (001) $\mathrm{SrTiO}_{3}$ substrates under different growth temperatures from 600 to $900{ }^{\circ} \mathrm{C}$. Substrates peaks are labeled in green, anatase peaks in blue. Vertical dashed lines are drawn to mark peak positions for comparison. 

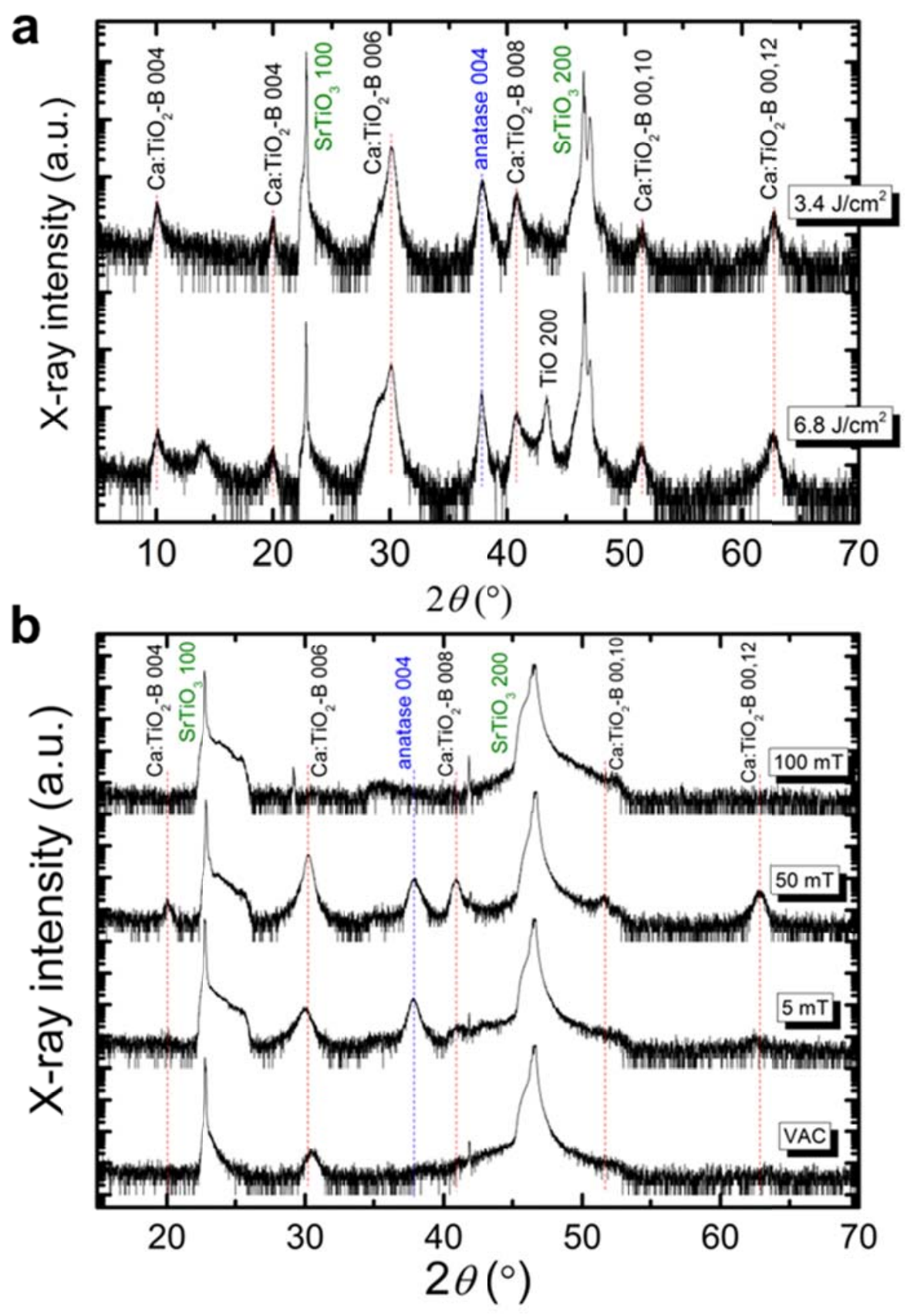

Figure 3.11 XRD patterns of the films deposited from a $10 \% \mathrm{CaO}$ target on (001) $\mathrm{SrTiO}_{3}$ substrates under different conditions: a) laser fluence from 3.4 to $6.8 \mathrm{~J} \mathrm{~cm}^{-2}$; b) $\mathrm{O}_{2}$ partial pressure from vacuum to 100 mTorr. Substrates peaks are labeled in green, anatase peaks in blue. Vertical dashed lines are drawn to mark peak positions for comparison. 


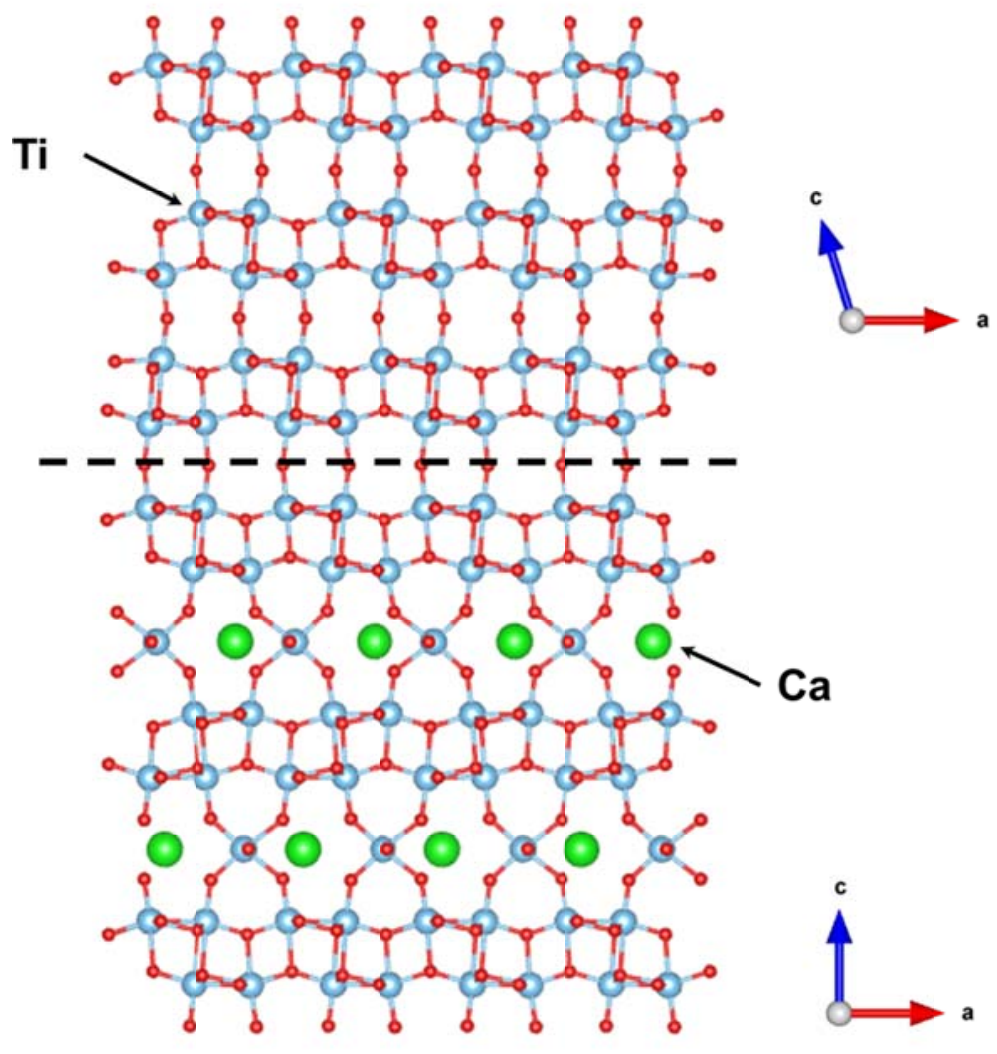

Figure 3.12 The atomic model projected along $\mathrm{Ca}$ : $\mathrm{TiO}_{2}-\mathrm{B}$ 's $b$-axis showing the epitaxial relationship between $\mathrm{TiO}_{2}-\mathrm{B}$ and $\mathrm{Ca}: \mathrm{TiO}_{2}-\mathrm{B}$. The dashed line indicates the interface between the two phases, where they align their respective (001) planes with each other. 

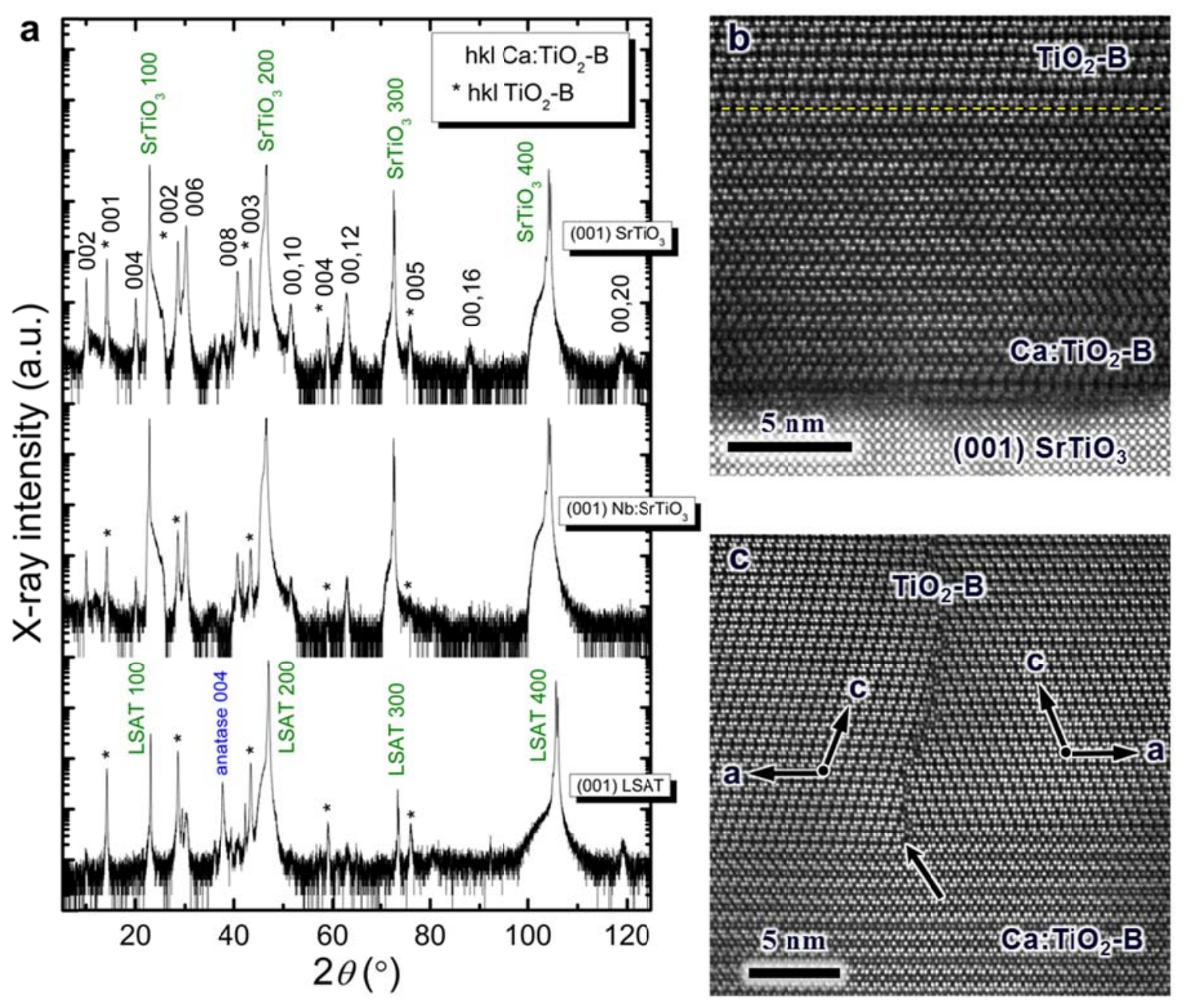

Figure $3.13(001) \mathrm{TiO}_{2}-\mathrm{B}$ grown on a (001) $\mathrm{Ca}: \mathrm{TiO}_{2}-\mathrm{B}$ template. a) XRD patterns of the dual layer films grown on various substrates. The $\mathrm{Ca}: \mathrm{TiO}_{2}-\mathrm{B}$ template was deposited from a $20 \% \mathrm{CaO}$ target. b-c) HAADF STEM images of a dual layer film grown on $\mathrm{SrTiO}_{3}(001)$ substrate. The dashed line in b) indicates the interface. [TEM image credit: Michael B. Katz] 

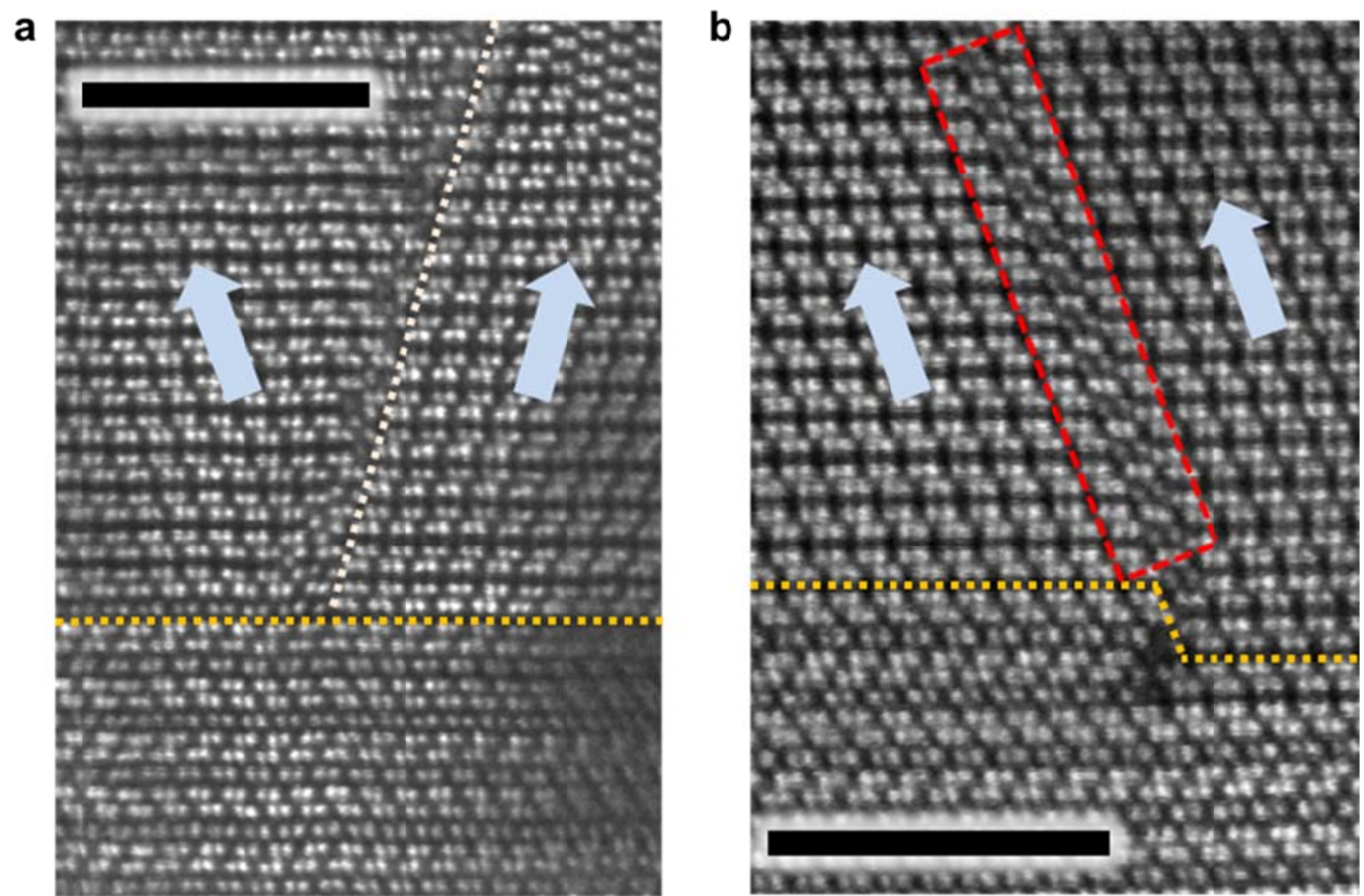

Figure 3.14 (a) High-resolution STEM images of defects at the interface between $\mathrm{TiO}_{2}-\mathrm{B}$ and $\mathrm{Ca}: \mathrm{TiO}_{2}-\mathrm{B}$ that generate the two adjacent $\mathrm{TiO}_{2}-\mathrm{B}$ grains orienting in opposite directions. (b) Two missing Ti-Ca layers result in the adjacent $\mathrm{TiO}_{2}-\mathrm{B}$ grains stacking in the same direction. Scale bars are $5 \mathrm{~nm}$. [TEM image credit: Sung Joo Kim] 

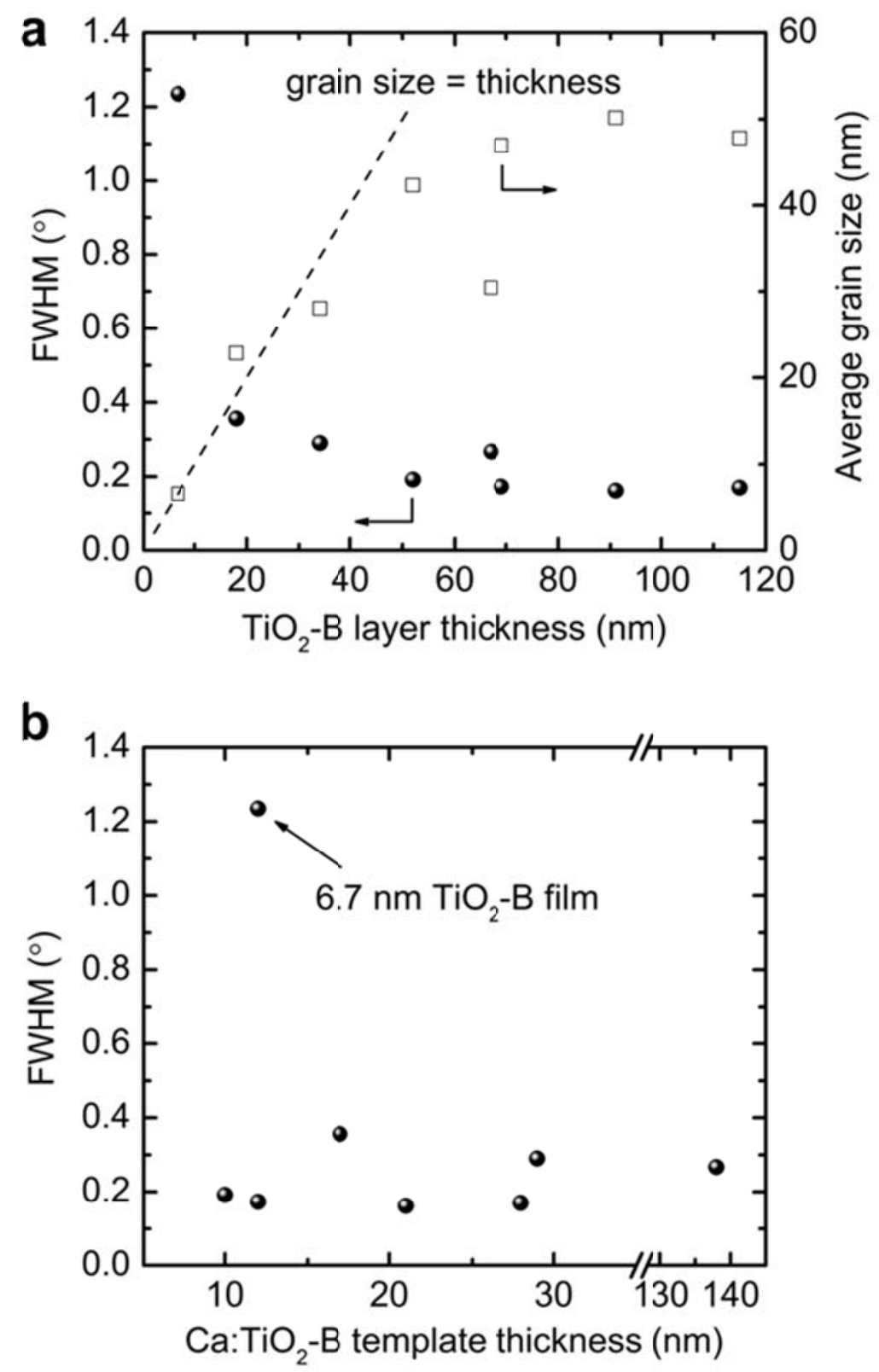

Figure 3.15 (a) FWHM of the $\mathrm{TiO}_{2}$-B 002 peak as a function of the layer thickness (solid dots), and the average grain size calculated from the FWHM using Scherrer equation (open squares). The dashed line indicates the situation when grain size equals film thickness. (b) $\mathrm{TiO}_{2}-\mathrm{B} 002$ FWHM as a function of the $\mathrm{Ca}: \mathrm{TiO}_{2}-\mathrm{B}$ template thickness. 


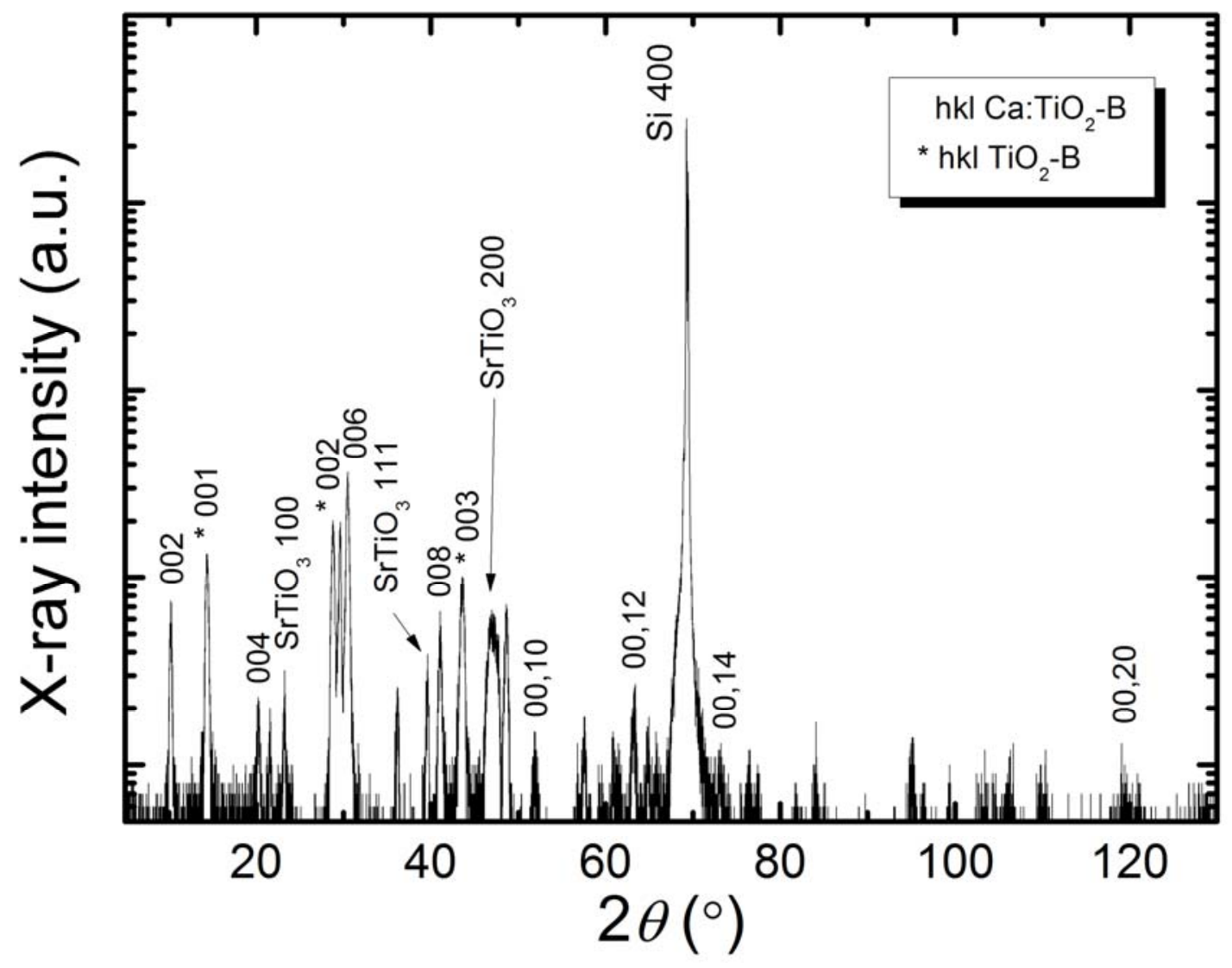

Figure 3.16 XRD $\theta-2 \theta$ pattern of a (001) $\mathrm{TiO}_{2}-\mathrm{B} / \mathrm{Ca}: \mathrm{TiO}_{2}-\mathrm{B}$ dual layer film grown on a $\mathrm{SrTiO}_{3}$ buffered (100) Si substrate. 


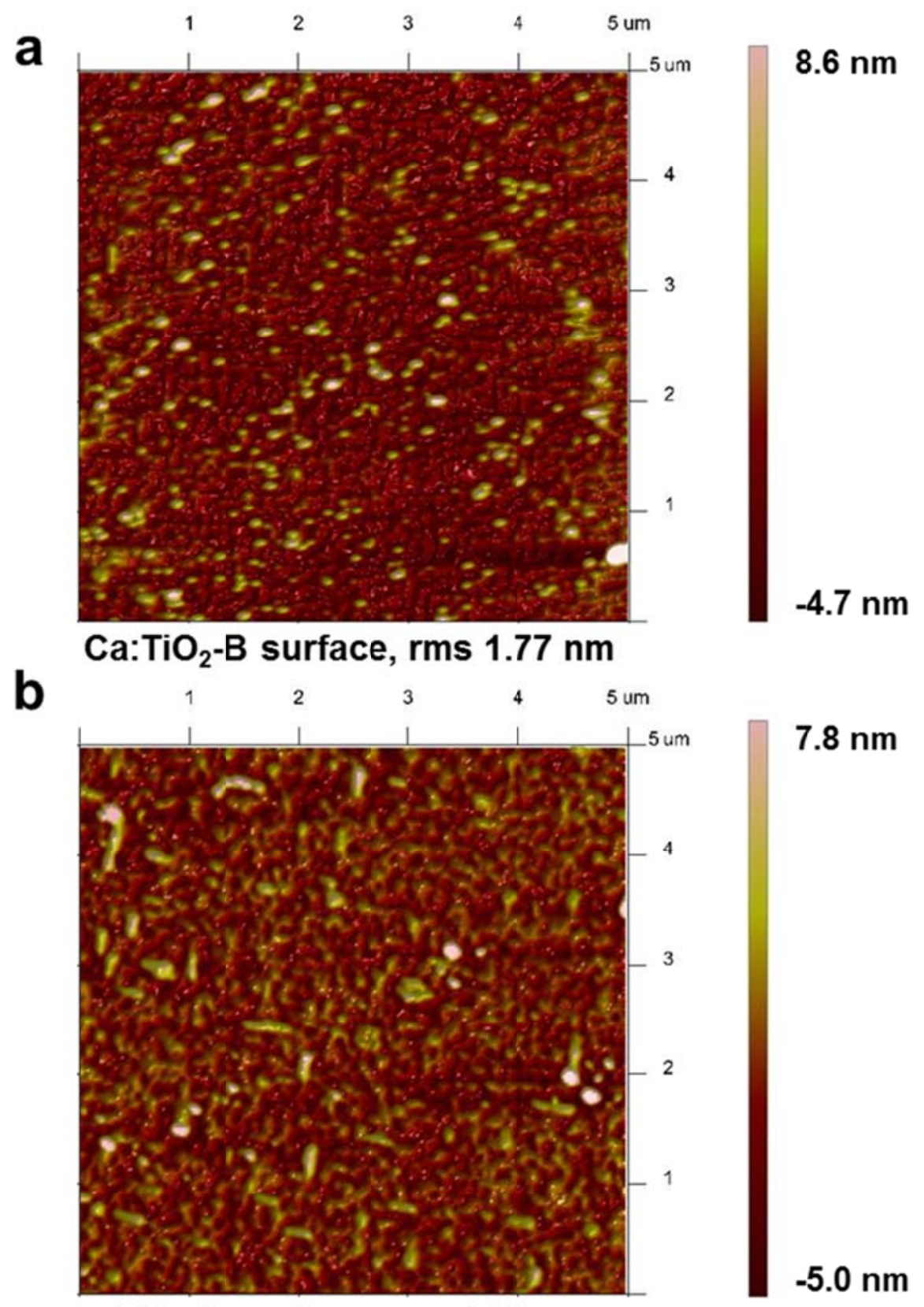

$\mathrm{TiO}_{2}$-B surface, rms $1.76 \mathrm{~nm}$

Figure 3.17 AFM images (tapping mode) showing the surfaces of (a) a $\mathrm{Ca}: \mathrm{TiO}_{2}-\mathrm{B}$ film and (b) a $\mathrm{TiO}_{2}$-B film (on top of a $\mathrm{Ca}: \mathrm{TiO}_{2}-\mathrm{B}$ template), respectively, both grown on (001) $\mathrm{SrTiO}_{3}$ substrates. Scan area is $5 \mu \mathrm{m} \times 5 \mu \mathrm{m}$ for both. 


\section{Chapter 4}

\section{Epitaxial Ca: $\mathrm{TiO}_{2}-\mathrm{B}\left(\mathrm{CaTi}_{5} \mathrm{O}_{11}\right)$ and $\mathrm{TiO}_{2}-\mathrm{B}$ Thin Films as Anode Materials for Lithium-Ion Batteries}

\subsection{Introduction}

Being an efficient energy storage device for portable or mobile electric systems, the battery is one of the very cornerstones that construct the modern way of life. There are two types of batteries. One is the primary battery, which can be used only once, such as the most common alkaline batteries. The other one is the secondary battery, which may be recharged for reuse, such as the well-known lead-acid, nickel-cadmium (Ni-Cd), nickel-metal-hydride (Ni-M-H), and lithium-ion batteries. The voltage of secondary batteries using aqueous electrolytes is limited by the stability of water ( $1.23 \mathrm{~V}$ vs. hydrogen reference), which may not sustain high voltage applications. $^{74,75}$ For instance, Ni-Cd and Ni-M-H batteries have a cell voltage $\sim 1.2 \mathrm{~V}$, and are often connected in series to produce a higher voltage. ${ }^{76}$

Rechargeable lithium-ion batteries (LIBs) offer several advantages when compared to traditional secondary power sources, including a wide range of cell voltages up to $>4 \mathrm{~V}$ depending on the particular cathode and anode materials used, ${ }^{77}$ high energy density, and long cycle life. ${ }^{78}$ Until most recently, lithium-ion batteries are considered to have no memory effect an effect commonly seen in Ni-Cd or Ni-M-H batteries which lose usable capacity owing to a reduced working voltage if recharged repeatedly after being only partially discharged, although the memory effect has so far only been observed in $\mathrm{LiFePO}_{4}{ }^{79}$ More generally, lithium-ion batteries are still far more superior in capacity retention over other rechargeable batteries. 
Besides enhancing the performance of a LIB in the three core areas, i.e. voltage $(P=I \times V)$, energy density (specific capacity) and cycle life (capacity retention), it is also crucial to improve the safety aspects of this high voltage energy storage system while using more earth abundant and low cost materials. In recent years, the focus of research in LIBs has expanded from developing capacity alone to enhancing charge/discharge rate capabilities, thus expanding the application to high power systems such as smart grids and electric vehicles. ${ }^{15,17}$

\subsubsection{The structure of a lithium-ion battery}

The structure of a LIB is essentially similar to other batteries. In many cases, a battery contains several basic cells to obtain the desired voltage and power rate. As shown in Figure 4.1, a cell is comprised of a cathode (positive electrode), an anode (negative electrode), and an electrolyte. The electrolyte, which can be a liquid or a solid, allows $\mathrm{Li}^{+}$movement but prohibits electron conduction. In the modern compact LIBs, where the cathode and the anode are closely packed together, an insulating separator is needed to prevent short-circuit.

During discharge, when the external circuit is powered by the electron flow, Li ions are extracted from the anode at the anode/electrolyte interface, and inserted into the cathode at the cathode/electrolyte interface, often reducing the metal ions in the cathode to a lower valence state. The most commonly used materials in a commercial LIB cell is $\mathrm{LiCoO}_{2}$ as cathode and graphite as anode, and the chemical reactions are:

$$
\begin{gathered}
\mathrm{LiCoO}_{2} \leftrightharpoons \mathrm{Li}_{1-x} \mathrm{CoO}_{2}+x \mathrm{Li}^{+}+x \mathrm{e}^{-} \\
6 \mathrm{C}+\mathrm{Li}^{+}+\mathrm{e}^{-} \leftrightharpoons \mathrm{LiC}_{6}
\end{gathered}
$$

The focus of this work is to develop titania-bronze as a high rate anode material. When characterizing a new battery material, it is often paired with Li metal as counter electrode in a battery cell, often called a half-cell, as compared to the notion of a full-cell such as the above. In this case, an anode material of interest actually works as the cathode vs. Li. Since the Li counter electrode acts as a zero potential supplier of $\mathrm{Li}^{+}$and a universal reference point, the material's 
electrochemical properties in a LIB is most accurately depicted. The voltage of a proposed full-cell can be easily predicted from the difference between the respective potentials of the cathode and the anode vs. lithium.

Most researchers deal with electrode materials synthesized in the powder form. The active material, which is the actual host to $\mathrm{Li}^{+}$, is usually mixed with carbon powders as current collector and a polymeric binder which holds the mixture together, and forming a slurry which is then pasted and dried on a piece of metal, usually $\mathrm{Cu}$ or Al, working as a base. Such an electrode is then assembled in a battery cell for testing.

In this thesis, where we work with crystalline thin films, the configuration, as well as testing protocols, is quite different from the traditional, and will be presented in detail later in this chapter.

\subsubsection{Electrode materials}

The pursuit of high cell voltage drove many researchers in the early days on the path searching for a cathode material with reversible $\mathrm{Li}^{+}$insertion and extraction capability. One of the earliest reports was in 1976 from Whittingham, introducing $\mathrm{TiS}_{2}$ as the positive electrode against lithium in a cell that worked at a voltage exceeding $2 \mathrm{~V}^{80}$ Unfortunately, Li was reactive to the electrolytes used the cell, where various flammable gas species were formed due to the decomposition of the electrolyte at its interface with $\mathrm{Li}^{81}$ creating a major safety issue that remains a concern in many battery systems even today. Li was used as the negative electrode, or anode, until the discovery of practical lithium-alloy, ${ }^{82}$ spinel based Li-transition metal oxides, ${ }^{83}$ and Li-carbon intercalation hosts. ${ }^{84}$

$\mathrm{TiS}_{2}$ was attractive at the time because it forms a layered, two-dimensional (2D) framework or a rigid three-dimensional structure $(3 \mathrm{D}) .{ }^{85,86}$, where the $2 \mathrm{D}$ polymorph provides better energy density. A shift in focus from sulfides to oxides started not long after, aiming at increasing the energy density of the material as well as the cell voltage. Similar to $\mathrm{TiS}_{2}$, some transition metal oxides also have a layered 2D form. As a result of higher electronegativity of the 
oxide anions as compared with those in the sulfides, the oxides have a higher ionization energy, and hence lead to a higher cell voltage when used as cathodes, ${ }^{87}$ as the cell voltage is determined by the difference in electron potential between the cathode and the anode.

The first transition metal oxides considered were known as host solids, which are materials synthesized without lithium in their framework. After the synthesis is complete, yielding an empty structure, the host solid is then lithiated by a Li intercalation process. Early host transition metal oxides investigated for $\mathrm{Li}^{+}$intercalation included $\mathrm{V}_{2} \mathrm{O}_{5}, \mathrm{MoO}_{3}, \mathrm{~V}_{6} \mathrm{O}_{13}$, ${ }^{88}$ $\mathrm{WO}_{3}$ and $\mathrm{MnO}_{2}{ }^{89}$

In 1980, Mizushima et al. discovered a new non-host type layered transition metal oxide with $\mathrm{Li}$ intercalation and deintercalation properties without destroying the oxide framework, i.e. $\mathrm{Li}_{x} \mathrm{CoO}_{2}(0<x \leq 1)$, which consists of $\mathrm{Li}^{+}$lamellae between two $\mathrm{CoO}_{2}$ lamellae, prepared by electrochemical extraction of lithium from the parent $\mathrm{LiCoO}_{2}$ ordered rock-salt structure. ${ }^{90}$ Due to its high average operating voltage of $\sim 3.7 \mathrm{~V}$ vs. lithium, high charge capacity $\left(274 \mathrm{~mA} \mathrm{~h} \mathrm{~g}{ }^{-1}\right.$ theoretical), ${ }^{91}$ and acceptable electrochemical stability, $\mathrm{LiCoO}_{2}$ is one of the most widely used cathode material in LIB technology today.

Because cobalt is an expensive metal, especially when considered for large scale applications, many other materials have been synthesized using alternate transition metals or mixtures of transition metals to vary the composition and properties of $\mathrm{LiCoO}_{2}$, while maintaining the layered $2 \mathrm{D}$ structure. These materials can be generally described as $\mathrm{Li} M \mathrm{O}_{2}$ and $\mathrm{Li}_{x} \mathrm{Co}_{1-x} \mathrm{O}_{2},{ }^{92-97}$ where $M$ represents the alternate transition metals such as $\mathrm{V}, \mathrm{Fe},{ }^{98,99} \mathrm{Ni},{ }^{100,101}$ and Mn. ${ }^{102-105}$ Metals (Mg) and non-metals (F) were also proposed in exchange for $\mathrm{Li}$ and $\mathrm{O}$, respectively, in $\mathrm{LiCoO}_{2}{ }^{106,107}$

The design of an anode material has essentially the same considerations as for the cathodes. High energy density, long cycle life, high rate performance, high structural stability and low cost are still the ultimate goals. The major difference, however, is that for anode materials, different voltage may be desired in different situations. Apparently, for a fixed cathode material, an anode with lower potential leads to a higher cell voltage and thus higher power, 
which is the very reason that graphite, with a potential of $0.1-0.2 \mathrm{~V}$ vs. $\mathrm{Li}$ and a capacity of 372 $\mathrm{mA} \mathrm{h} \mathrm{g}{ }^{-1}$, is the most commonly used commercial anode material. On the other hand, the low potential introduces an inherent safety issue: lithium plating. The lower the potential of a material is vs. $\mathrm{Li}$, the more easily $\mathrm{Li}$ would plate on the surface of that material. To make things worse, the electro-deposition of Li occurs in the form of dendritic growth, as shown in Figure 4.2. Once the Li dendrite pierces through the separator and reaches the cathode to cause short-circuit, the catastrophic failure of the battery may end with a fire or an explosion. Low anode operating voltage $(<1 \mathrm{~V})$ also causes the electrolyte to decompose and form an unstable SEI layer on the anode's surface, releasing gases that build pressure in the cell and endangering the safety of the battery system especially during prolonged cycling. Therefore, when the safety and stability are considered as priority over energy/power density, ${ }^{108}$ higher anode operating voltage is factually a beneficial factor over graphite. Although the sacrifice in full-cell voltage may be disadvantageous in portable systems, high voltage anodes are excellent candidates for stationary energy storage systems that have less stringent weight and space requirements but emphasize operation safety, such as grid level applications.

Table 4.1 and Table 4.2 list a few popular cathode and anode materials, respectively, comparing their properties. The $\mathrm{TiO}_{2}-\mathrm{B}$ and $\mathrm{Ca}: \mathrm{TiO}_{2}-\mathrm{B}$ thin films presented in this thesis are also included (Table 4.2).

For any electrode material, including both the cathode and the anode, the major problem with layered transition metal oxides is that, they usually have other more stable phases, e.g. the spinel phase for $\mathrm{LiCoO}_{2}$, or anatase for $\mathrm{TiO}_{2}$. The open structure, which is the very reason for their high $\mathrm{Li}^{+}$capacity or fast $\mathrm{Li}^{+}$transport, is also the weakness of the framework, with the risk of collapsing into a more stable polymorph upon $\mathrm{Li}^{+}$cycling. Both in synthesis and the lithiation-delithiation process, there is always a concern to maintain the structural stability as well as the full recharge capacity. Another issue with transition metal oxides used as LIB materials is that they often have poor electronic conductivity. Lithium transition metal oxides are usually $p$-type semiconductors. ${ }^{109,110}$ Tukamoto et al. demonstrated that $\mathrm{Mg}$-doping into $\mathrm{LiCoO}_{2}$ 
to form $\mathrm{LiMg}_{x} \mathrm{Co}_{1-x} \mathrm{O}_{2}$ increased the conductivity by over two orders of magnitude, by partial substitution of $\mathrm{Co}^{3+}$ by $\mathrm{Mg}^{2+}$ and compensating hole creation, at the sacrifice of a small reduction in capacity associated with a reduction in $\mathrm{Co}^{3+}$ content. ${ }^{109}$ It was also shown that $\mathrm{LiCo}_{1-x} \mathrm{Ni}_{x} \mathrm{O}_{2}$ has higher electronic conductivity than $\mathrm{LiCoO}_{2},{ }^{111-113}$ again at the cost of a loss in energy capacity. More generally, manipulating the electronic band structure by doping is the main approach to enhance conductivity in battery materials, both for cathodes and for anodes.

\subsection{3 $\mathrm{Ca}: \mathrm{TiO}_{2}-\mathrm{B}$ and $\mathrm{TiO}_{2}-\mathrm{B}$ as anode materials}

The $\mathrm{TiO}_{2}-\mathrm{B}$ and $\mathrm{Ca}: \mathrm{TiO}_{2}-\mathrm{B}$ materials discussed in this thesis are high voltage anode materials with a buffer $>1.5 \mathrm{~V}$ before lithium plating, offering good inherent safety in a battery. The loss in voltage may be compensated by their high rate capabilities, since high rate stands for large charge/discharge current, which is the other defining factor in power output $(P=I \times V)$; and by its low cost, minimal environmental impact and structural stability, which are all important aspects in commercialization.

As introduced in Section 3.1, $\mathrm{TiO}_{2}-\mathrm{B}$ has been synthesized in various forms of nanostructures over the past decade and tested for its electrochemical properties, many approaching its theoretical $\mathrm{Li}^{+}$capacity of $335 \mathrm{~mA} \mathrm{~h} \mathrm{~g}^{-1}$. For example, the Bruce group (2005) reported a specific capacity of $305 \mathrm{~mA} \mathrm{~h} \mathrm{~g}^{-1}\left(\mathrm{Li}_{0.91} \mathrm{TiO}_{2}\right)$ in $\mathrm{TiO}_{2}-\mathrm{B}$ nanowires, ${ }^{33}$ and $328 \mathrm{~mA} \mathrm{~h}$ $\mathrm{g}^{-1}\left(\mathrm{Li}_{0.98} \mathrm{TiO}_{2}\right)$ in $\mathrm{TiO}_{2}-\mathrm{B}$ nanotubes. ${ }^{21}$ As the pseudocapacitive behavior of lithium storage in $\mathrm{TiO}_{2}-\mathrm{B}$ became known, ${ }^{16,38}$ the research community started to pay more attention to its performance at high charge/discharge rates, witnessed by a few representative reports in recent years. Mesoporous $\mathrm{TiO}_{2}-\mathrm{B}$ microspheres prepared by Liu et al. (2011) showed a capacity of 165 $\mathrm{mA} \mathrm{h} \mathrm{g} \mathrm{g}^{-1}$ at $10 \mathrm{C}$ and $116 \mathrm{~mA} \mathrm{~h} \mathrm{~g}^{-1}$ at $60 \mathrm{C}$, with exceptional retention after 5000 cycles. ${ }^{23}$ Nanosheet-constructed porous $\mathrm{TiO}_{2}$-B synthesized by Liu et al. (2012) exhibited a capacity of $216 \mathrm{~mA} \mathrm{~h} \mathrm{~g}^{-1}$ at $10 \mathrm{C}$, with a capacity loss of $7.4 \%$ after 200 cycles. ${ }^{24}$ A flexible film electrode made by anchoring $\mathrm{TiO}_{2}-\mathrm{B}$ nanosheets on activated carbon fabric, which was fabricated by Liu $e t$ al. (2013) delivered a capacity of $130 \mathrm{~mA} \mathrm{~h} \mathrm{~g}^{-1}$ after 2000 cycles at 20C. ${ }^{35}$ Chemically bonded 
$\mathrm{TiO}_{2}-\mathrm{B}$ nanosheet/reduced graphene oxide hybrid electrode prepared by Etacheri et al. (2014) showed a high specific capacity of $200 \mathrm{~mA} \mathrm{~h} \mathrm{~g}^{-1}$ at $40 \mathrm{C}$, maintaining $80 \%$ of the initial capacity after 1000 cycles. ${ }^{114}$ Commonly seen in many of these reports, the charge-discharge voltage profiles of the $\mathrm{TiO}_{2}$-B based electrodes are usually in the characteristic sloping shape, which is associated with the pseudocapacitive process, and often contain a plateau/plateaus at 1.6-2.2 V probably attributable to $\mathrm{TiO}_{2}$-anantase impurities. ${ }^{23,24}$

As a variant phase to $\mathrm{TiO}_{2}-\mathrm{B}, \mathrm{Ca}: \mathrm{TiO}_{2}-\mathrm{B}$ is expected to have similar lithium electrochemical reactivity. In the following sections, (001) $\mathrm{Ca}: \mathrm{TiO}_{2}-\mathrm{B}$ and (001) $\mathrm{TiO}_{2}-\mathrm{B}$ (on a thin $\mathrm{Ca}: \mathrm{TiO}_{2}-\mathrm{B}$ template layer) thin films grown on (001) $\mathrm{SrTiO}_{3}$ substrates are studied as anode materials for LIBs in battery half-cells. Both the $\mathrm{Ca}: \mathrm{TiO}_{2}-\mathrm{B}$ films and the $\mathrm{TiO}_{2}-\mathrm{B} / \mathrm{Ca}: \mathrm{TiO}_{2}-\mathrm{B}$ dual layer films chosen for this study have fairly smooth surfaces on the $a-b$ plane due to the characteristically layered structure of bronze, as shown in Figure 4.3. The films are generally of high crystalline quality, although crystal defects including grain boundaries, dislocations and stacking faults can be identified, nevertheless with a low density. More detailed investigation into various structural features and forming mechanism in these thin films can be found in Ref. 71. The exposed (001) surface of the test films is the predominate interface with the electrolyte for $\mathrm{Li}^{+}$exchange, since the other crystal facets on the side of the film can be neglected as the thicknesses of the films $(50-200 \mathrm{~nm})$ are orders of magnitude smaller than the length and width $(5-10 \mathrm{~mm})$ constituting the surface area, imposing great certainty in data interpretation.

\subsection{Electrochemical measurement setup for the bronze films}

\subsubsection{Bronze thin film preparation on $\mathrm{Nb}: \mathrm{SrTiO}_{3}$ substrates}

Epitaxial (001) $\mathrm{Ca}: \mathrm{TiO}_{2}-\mathrm{B}$ thin films grown on (001) $\mathrm{SrTiO}_{3}$ substrates were first synthesized using a $\mathrm{CaTi}_{4} \mathrm{O}_{9}$ (nominal composition) target made by mixing $80 \% \mathrm{TiO}_{2}$ and $20 \%$ $\mathrm{CaO}$ powders, sintering at $1400{ }^{\circ} \mathrm{C}$, and pressed into a pellet under $10,000-\mathrm{lb}$ of force. The laser fluence was $\sim 3.4 \mathrm{~J} \mathrm{~cm}^{-2}$ at a $10 \mathrm{~Hz}$ repetition rate, and the substrate-target distance was set to $6.35 \mathrm{~cm}$. The films were deposited at $800{ }^{\circ} \mathrm{C}$ in an oxygen ambient of 0.05 Torr. The 
deposition rate was $0.01-0.02 \AA /$ pulse. Films studied in this work have thicknesses of $50-200 \mathrm{~nm}$ (typical deposition time of 1-4 hours), which were measured by a Veeco Dektak profilometer and confirmed with TEM images.

Figure 4.4(a) shows the $\theta-2 \theta$ XRD pattern of the epitaxial (001) thin film of the $\mathrm{Ca}: \mathrm{TiO}_{2}-\mathrm{B}$ phase, where the film peaks are very strong and sharp, confirming a high quality, single crystalline film. No other impurity phases or polycrystalline $\mathrm{Ca}: \mathrm{TiO}_{2}-\mathrm{B}$ peaks were detected. A HAADF STEM image taken along the $b$ direction of the structure is displayed in Figure 4.4(b), showing the interesting layered, zigzag pattern where every inserted layer of alternating $\mathrm{Ti}$ and $\mathrm{Ca}$ atoms flips the stacking direction of the next two layers of $\mathrm{Ti}$ atoms above it, consistent with the atomic model in Figure 1.1. Figure 4.4(c) and (d) show the $\theta-2 \theta$ XRD pattern and the HAADF image, respectively, of the (001) $\mathrm{TiO}_{2}-\mathrm{B} / \mathrm{Ca}: \mathrm{TiO}_{2}-\mathrm{B}$ dual layer structure.

In order to investigate the electrochemical performance of the thin films, a current collector is needed. One natural approach was to grow the films on conductive $\mathrm{SrTiO}_{3}$ substrates doped with 0.5 at.\% $\mathrm{Nb}$ (resistivity $0.05 \Omega \mathrm{cm}$ ), which served as bottom current collectors. However, we have found that the crystal quality of the films grown on (001) $\mathrm{Nb}: \mathrm{SrTiO}_{3}$ substrates varies from growth to growth, despite the fixed conditions. Sometimes similar film quality to the ones grown on undoped $\mathrm{SrTiO}_{3}$ substrates could be achieved, while other times the films contained significant amount of anatase grains at the interface with the substrate, as shown in Figure 4.5. Although the reason is yet unclear, it should be associated with certain effects on the surface of the $\mathrm{Nb}: \mathrm{SrTiO}_{3}$ substrate. Possible explanations include different ion bonding dynamics between the film and the substrate through electron cloud overlapping, due to the different electrostatic boundary conditions at the surface of a conductive crystal from that of a non-conductive crystal; $\mathrm{Nb}$ dopant migrating within the $\mathrm{SrTiO}_{3}$ host matrix, resulting in lattice deformation near the substrate surface; surface dulling or roughening due to in-air storage, etc. It was also noticed that some films grown on $\mathrm{Nb}: \mathrm{SrTiO}_{3}$ were delaminated from the substrate during TEM sample preparation (grinding and polishing) more easily than those grown on undoped $\mathrm{SrTiO}_{3}$. Even different batches of $\mathrm{Nb}: \mathrm{SrTiO}_{3}$ substrates, though purchased from the 
same supplier (MTI Corporation), yielded different results of $\mathrm{Ca}: \mathrm{TiO}_{2}-\mathrm{B}$ film deposition.

An effective way to increase the success rate of $\mathrm{Ca}: \mathrm{TiO}_{2}-\mathrm{B}$ growth on $\mathrm{Nb}: \mathrm{SrTiO}_{3}$, while improving the film quality, is to add a $\mathrm{SrTiO}_{3}$ intervening layer on the surface of the substrate. Figure 4.6 shows such a film, where a $9 \mathrm{~nm}$ thick $\mathrm{SrTiO}_{3}$ layer was homoepitaxially deposited on the substrate by using a stoichiometric $\mathrm{SrTiO}_{3}$ target and the same growth conditions as for the bronze film $\left(\mathrm{SrTiO}_{3}\right.$ can be grown over a wide range of oxidizing environment), before depositing the $\mathrm{Ca}: \mathrm{TiO}_{2}-\mathrm{B}$ layer. The growth can either end with a $\mathrm{Ca}: \mathrm{TiO}_{2}-\mathrm{B}$ film, or further with subsequent growth of a $\mathrm{TiO}_{2}-\mathrm{B}$ film above the $\mathrm{Ca}: \mathrm{TiO}_{2}-\mathrm{B}$ template (Figure 4.6(a)). The $\mathrm{SrTiO}_{3}$ buffer layer obviously improves the surface conditions, leading to high quality bronze crystals on top (Figure 4.6(b)).

Although adopting the $\mathrm{SrTiO}_{3}$ buffer layer solves the problem of bronze film growth, it introduces new issues at the same time. Pure $\mathrm{SrTiO}_{3}$ is highly insulating, which, even with the thickness of merely a few nanometers, creates a high energy barrier for electrons to move through, thus defeating the purpose of using $\mathrm{Nb}: \mathrm{SrTiO}_{3}$ substrates as current collector in a battery. We have found that such an approach may work for slow battery cycling experiments under low currents, but the performance is greatly limited in high rate cycling tests. One possible remedy is to fabricate a Nb:SrTiO 3 buffer layer instead of the insulating $\mathrm{SrTiO}_{3}$ buffer. However there was no guarantee that this might be effective, since it was unknown whether the root cause of the bronze growth problem was with the substrate surface or the $\mathrm{Nb}: \mathrm{SrTiO}_{3}$ material itself. Hence, such solution was not tried, while instead, a top current collection configuration has been devised, as follows

\subsubsection{Top current collection configuration and data acquisition protocol}

For films grown on non-conductive $\mathrm{SrTiO}_{3}$ substrates, a top current collection geometry was fabricated using a grid of $\mathrm{Cu}$ wires with line width of $100 \mu \mathrm{m}$ and a thickness of $20 \mathrm{~nm}$ which were deposited on the film surface in an E-beam evaporator with a Mo mask. On a $10 \times 10$

$\mathrm{mm}^{2}$ test sample, the grid covers $<0.8 \%$ of the surface area, which should have a negligible 
influence on the $\mathrm{Li}^{+}$exchange between the film and the electrolyte. Since the film surface needs to be facing the separator, the $\mathrm{Cu}$ wire grid should be connected to the back of the sample, which touches the stainless steel casing of the test cell that links to the external circuit. A thin $\mathrm{Cu}$ foil ( $0.025 \mathrm{~mm}$ thick) was wrapped around the sample, with edges touching the ends of the $\mathrm{Cu}$ wires on top, and a good connection is formed when the entire cell stack is pressed firmly together. Such a configuration is shown schematically in Figure 4.7, which presents the situation that a $\mathrm{TiO}_{2}-\mathrm{B} / \mathrm{Ca}: \mathrm{TiO}_{2}-\mathrm{B}$ dual layer film is studied. Obviously when only the $\mathrm{Ca}: \mathrm{TiO}_{2}-\mathrm{B}$ is of interest, the $\mathrm{TiO}_{2}$-B layer is absent.

To study the electrochemical properties of such a small amount of active material is in itself a significant challenge. A $50 \mathrm{~nm} \mathrm{Ca:TiO} 2-\mathrm{B}$ thin film on a $10 \times 10 \mathrm{~mm}^{2}$ substrate weighs only $\sim 18 \mu \mathrm{g}$, compared to tens of mg of the active material commonly used in a slurry electrode for conventional battery studies reported in the literature. Therefore, a new data acquisition protocol is demanded to assure the validity of the data recorded from these test cells. Since we have two different testing configurations, i.e. bottom current collection $\left(\mathrm{Nb}: \mathrm{SrTiO}_{3}\right.$ substrate) and top current collection ( $\mathrm{Cu}$ wire grid), we may acquire experimental data on the same control samples using both techniques and compare the results, so that they may validate each other.

While using conductive $\mathrm{Nb}: \mathrm{SrTiO}_{3}$ substrate as bottom current collector, electrons are traveling through the entire substrate to the external circuit, and therefore the electrochemical force may drive some $\mathrm{Li}^{+}$into the substrate. Even though $\mathrm{SrTiO}_{3}$ does not appear to have a high $\mathrm{Li}^{+}$capacity, ${ }^{115}$ it is important to rule out the contribution from the substrate for determining the actual capacity of the film. A bare $\mathrm{Nb}: \mathrm{SrTiO}_{3}$ substrate was assembled in a half-cell and tested with exactly the same routine and rates as for the thin film samples. The measured capacity of the substrate at each rate was then subtracted from the total to obtain the capacity of the film at that rate. It should be noted that the voltage window of 1-3 V for bronze film testing is much higher than the possible $\mathrm{Li}$ intercalation voltage of $\mathrm{SrTiO}_{3}(0.07-0.18 \mathrm{~V}),{ }^{115}$ so the substrate contribution is very low, as seen in the control test at a $1 \mathrm{C}$ rate below in Figure 4.8. Based on these results, the electrical charge contribution from a $10 \times 10 \times 0.5 \mathrm{~mm}^{3} \mathrm{Nb}: \mathrm{SrTiO}_{3}$ substrate is 
$\sim 0.004 \mathrm{~mA} \mathrm{~h}$, while the charge capacity of a $50 \mathrm{~nm} \mathrm{Ca:TiO}{ }_{2}-\mathrm{B}$ film is $\sim 0.005 \mathrm{~mA} \mathrm{~h}$. Therefore, the 'signal-to-noise' ratio is good enough to assure the accuracy of the capacity data acquired from the film itself, after subtracting the contribution from the substrate.

A similar approach is needed to determine the capacity of the regular $\mathrm{TiO}_{2}-\mathrm{B}$ phase. Since the $\mathrm{TiO}_{2}-\mathrm{B}$ film has to be grown on top of a $\mathrm{Ca}: \mathrm{TiO}_{2}-\mathrm{B}$ template layer, our procedure for figuring its specific capacity was to cycle the $\mathrm{Ca}: \mathrm{TiO}_{2}-\mathrm{B}$ sample and the $\mathrm{TiO}_{2}-\mathrm{B} / \mathrm{Ca}: \mathrm{TiO}_{2}-\mathrm{B}$ dual layer sample of the same sizes using exactly the same routine and rates, determine the specific capacity of $\mathrm{Ca}: \mathrm{TiO}_{2}-\mathrm{B}$ at each rate first, calculate the capacity contribution of the $\mathrm{Ca}: \mathrm{TiO}_{2}-\mathrm{B}$ layer in the dual layer sample from its thickness obtained by STEM, and finally subtract that part as well from the total capacity.

For thin films grown on insulating $\mathrm{SrTiO}_{3}$ substrates and using the top current collection configuration, the substrate is not a part of the electrochemical reaction or the circuit, so its contribution to the measured capacity should be minimal and hence was not considered. This method provides additional convenience in cyclic voltammetry $(\mathrm{CV})$ measurements, where the contribution from the substrate cannot be easily subtracted.

For the purpose of comparing the effectiveness of these two configurations described above, two $\mathrm{TiO}_{2}-\mathrm{B} / \mathrm{Ca}: \mathrm{TiO}_{2}-\mathrm{B}$ dual layer control samples were grown simultaneously to the same thicknesses on a (001) $\mathrm{SrTiO}_{3}$ substrate and a (001) 0.5 at.\% $\mathrm{Nb}: \mathrm{SrTiO}_{3}$ substrate, respectively. XRD and TEM results confirmed that the two films were of almost equal quality. The former was processed in a top current collection geometry as in Figure 4.7. Both samples, together with a bare $\mathrm{Nb}: \mathrm{SrTiO}_{3}$ substrate, were then assembled in half-cells with metallic Li counter electrodes and tested under $1 \mathrm{C}$ and $10 \mathrm{C}$ rates for 100 cycles. The discharge capacities of $\mathrm{TiO}_{2}-\mathrm{B}$ obtained in the two samples are compared in Figure 4.9. It is clear that the two test configurations produced similar results, while the capacity values acquired from the top $\mathrm{Cu}$ grid method were slightly higher. This is due to the fact that $\mathrm{Cu}$ metal provides better current collection efficiency than $\mathrm{Nb}: \mathrm{SrTiO}_{3}$ semiconductor substrate. Such an advantage will become more significant as the rates increase beyond 10C. Therefore, results of battery cycling performance reported later in this 
thesis were all acquired in the top $\mathrm{Cu}$ grid collector configuration.

Recent studies demonstrated that Li plating may occur at the interface of electrolyte and $\mathrm{Cu}$ current collector, ${ }^{116}$ which would result in erroneously higher capacity measured for the Li storage material in battery cycling tests. To rule out the influence of possible Li plating on the $\mathrm{Cu}$ grid, a $\mathrm{Ca}: \mathrm{TiO}_{2}-\mathrm{B}$ thin film sample with top $\mathrm{Cu}$ grid current collector was charged and discharged at the rate of $1000 \mathrm{C}$ for 20 cycles, taken out of the cell at a half cycle when the film was fully charged with $\mathrm{Li}^{+}$, and examined with an FEI Quanta scanning electron microscope. The surface image and the $\mathrm{X}$-ray energy dispersive spectrum from the $\mathrm{Cu}$ wire are shown in Figure 4.10. No obvious $\mathrm{Li}$ dendrite formation was observed either on the $\mathrm{Cu}$ wire or on the $\mathrm{Ca}: \mathrm{TiO}_{2}-\mathrm{B}$ film surface.

By this point, we have established confidence in the validity of the testing methods and the results that will be discussed for the bronze films below. To calculate specific capacity for both the $\mathrm{Ca}: \mathrm{TiO}_{2}-\mathrm{B}$ and $\mathrm{TiO}_{2}-\mathrm{B}$ phases, the mass of active material was determined from its theoretical density, measured surface area and thickness. The mass loading of active material was $\sim 0.036 \mathrm{mg} / \mathrm{cm}^{2}$.

\subsection{Cycling performance of the (001) $\mathrm{Ca}^{\mathrm{T}} \mathrm{TiO}_{2}-\mathrm{B}$ films}

In $\mathrm{Ca}: \mathrm{TiO}_{2}-\mathrm{B}$, assuming $5 \mathrm{Li}^{+}$is intercalated per $\mathrm{CaTi}_{5} \mathrm{O}_{11}$ formula unit, reducing all $\mathrm{Ti}^{4+}$ to $3^{+}$, its theoretical capacity is estimated to be $294 \mathrm{~mA} \mathrm{~h} \mathrm{~g}^{-1}$. We have thus far achieved in these (001) $\mathrm{Ca}_{\mathrm{TiO}_{2}}-\mathrm{B}$ films a capacity of $273 \mathrm{~mA} \mathrm{~h} \mathrm{~g} \mathrm{~g}^{-1}$, corresponding to a composition of $\mathrm{Li}_{4.64} \mathrm{CaTi}_{5} \mathrm{O}_{11}$ at the end of discharge, at a rate of $33.5 \mathrm{~mA} \mathrm{~g}^{-1}$, favorable when compared to other non-graphitic anodes currently being studied. Typical charge/discharge voltage profiles are shown in Figure 4.11, where the $\mathrm{Ca}: \mathrm{TiO}_{2}-\mathrm{B}$ film exhibit sloped profiles corresponding to a pseudocapacitive process of $\mathrm{Li}^{+}$transport, which is a typical characteristic often observed for $\mathrm{TiO}_{2}-\mathrm{B}{ }^{24,26,117}$ These results also support that both the $\mathrm{Ca}: \mathrm{TiO}_{2}-\mathrm{B}$ films have good purity without a pronounced amount of other $\mathrm{TiO}_{2}$ polymorphs, which would otherwise create plateaus in the profiles. ${ }^{24}$ Theoretical calculations show that up to $18 \mathrm{Li}$ sites may be filled during 
intercalation in a $\mathrm{Ca}_{4} \mathrm{Ti}_{20} \mathrm{O}_{44}$ unit cell, ${ }^{118}$ corresponding to a composition of $\mathrm{Li}_{4.5} \mathrm{CaTi}_{5} \mathrm{O}_{11}$, which is in good agreement with the experimental data.

The term 'pseudocapacitance' stems from the characteristically sloped voltage profiles, observed in a few materials including $\mathrm{TiO}_{2}-\mathrm{B}$ and $T-\mathrm{Nb}_{2} \mathrm{O}_{5}{ }^{17}$ In a constant-current experiment, the cell potential $E$ changes with the extent of charge $Q$ :

$$
Q=C \Delta E
$$

where $Q$ is the charge passed (Coulombs), $\Delta E$ is the potential change (V) and $C$ is the pseudocapacitance (F). Apparently for a standard capacitor, where $C$ is a constant, the voltage profile is a straight line with a fixed slope. However, a capacitor cannot have a high capacity since it only stores electrical charge on the surface. Pseudocapacitive battery materials, on the other hand, have the ability to store energy in the bulk, and to release and restore that energy in a process much faster than traditional battery materials, at ultrahigh rates comparable to those of supercapacitors (high power delivery). ${ }^{15}$ The charge/discharge voltage profiles of these materials are thus shaped in between the cases of a capacitor and a conventional battery, as schematically shown in Figure 4.12. Presently, the prevailing point of view for pseudocapacitive behavior is that they usually emerge in materials with a loose, layered structure, where facile $2 \mathrm{D}^{+}{ }^{+}$ diffusion pathways exist without much obstruction, so the atomic structure does not undergo phase transformations on $\mathrm{Li}^{+}$intercalation. ${ }^{17}$ These 2D pathways, often called open channels, may act as secondary surfaces, so the charge storage that behaves as a quasi-2D process exhibits similar behavior to $2 \mathrm{D}$ surface adsorption reactions, ${ }^{119}$ and hence the sloped voltage profiles which are typically associated with surface effects.

Capacity retention has been examined for this material. 10C $\left(1 \mathrm{C}=294 \mathrm{~mA} \mathrm{~g}^{-1}\right)$ cycling results in 100 cycles are shown in Figure 4.13. The capacity loss and the lower Coulombic efficiency in the first 20 cycles reflect the poor electrical conductivity characteristic of pure $\mathrm{Ca}: \mathrm{TiO}_{2}-\mathrm{B}{ }^{33}$ From cycle 20 to 200 , however, the loss was only $0.2 \%$ per cycle, and the Coulombic efficiency close to 1 . The major cause for the capacity loss is the much longer $\mathrm{Li}^{+}$and $\mathrm{e}^{-}$transport paths in the bulk of the film than in typical powder particles or nanostructures often 
seen the literature reports.

\subsection{Cycling performance of the (001) $\mathrm{TiO}_{2}-\mathrm{B}$ films}

As explained earlier, since the regular $\mathrm{TiO}_{2}-\mathrm{B}$ films needed to be grown on a $\mathrm{Ca}: \mathrm{TiO}_{2}-\mathrm{B}$ template, the capacity of the $\mathrm{TiO}_{2}$-B film was calculated by subtracting the contribution from the $\mathrm{Ca}: \mathrm{TiO}_{2}-\mathrm{B}$ layer. The samples were processed in the top current collection geometry (Figure 4.7). The insertion reaction of $\mathrm{Li}^{+}$into $\mathrm{TiO}_{2}$, regardless of various polymorphs, can be expressed as: ${ }^{120}$

$$
\mathrm{TiO}_{2}+x \mathrm{Li}^{+}+x \mathrm{e}^{-} \leftrightharpoons \mathrm{Li}_{x} \mathrm{TiO}_{2}
$$

The charge/discharge voltage profiles of a (001) $\mathrm{TiO}_{2}-\mathrm{B}$ thin film at a rate of $\mathrm{C} / 10$ $\left(1 \mathrm{C}=335 \mathrm{~mA} \mathrm{~g}^{-1}\right)$ is shown in Figure 4.14(a), again in a sloped shape, similarly to those of $\mathrm{Ca}: \mathrm{TiO}_{2}-\mathrm{B}$. The film almost discharged to its theoretical capacity, recording $334 \mathrm{~mA} \mathrm{~h} \mathrm{~g}^{-1}$ at the end of discharge, corresponding to a $\mathrm{Li}$ content of $\mathrm{Li}_{0.997} \mathrm{TiO}_{2}$. Capacity retention was examined at a slow rate of $\mathrm{C} / 12$, and the film maintained a high capacity close to $300 \mathrm{~mA} \mathrm{~h} \mathrm{~g}^{-1}$ after 18 cycles, as exhibited in Figure 4.14(b). The capacity loss was again caused by the poor electrical conductivity of the $\mathrm{TiO}_{2}$-B material.

Cyclic voltammograms (CV curves) of the $\mathrm{TiO}_{2}-\mathrm{B}$ thin film sample were recorded at scan rates from 0.1 to $1 \mathrm{mV} \mathrm{s}^{-1}$ as shown in Figure 4.15(a). A pair of redox peaks at $1.54 \mathrm{~V}$ and $1.69 \mathrm{~V}$ was observed, which represents the signature pseudocapacitive $\mathrm{Li}^{+}$storage behavior of $\mathrm{TiO}_{2}-\mathrm{B} \cdot{ }^{17,24}$ No other redox peaks can be identified on the $\mathrm{CV}$ curves, confirming the excellent phase purity of the $\mathrm{TiO}_{2}-\mathrm{B}$ film. ${ }^{23,35}$ The $\mathrm{CV}$ curves encompass a larger area at a higher voltage scan rate. The change in the shape and encompassed area of the CV curves originates from the pseudocapacitive charging process, which is a specific characteristic of $\mathrm{TiO}_{2}-\mathrm{B}$, as described below: ${ }^{16,17}$

$$
i=\frac{\mathrm{d} Q}{\mathrm{~d} t}=C \frac{\mathrm{d} E}{\mathrm{~d} t}=C v
$$

where $v$ is the scan rate and $C$ is the pseudocapacitance. Therefore as the scan rate increases, the $\mathrm{CV}$ loops grow bigger. Other than the areas these curves encompass, the shapes of the CV curves 
(Figure 4.15(a)) are actually similar at different scan rates.

The slight shift of redox peak position with increasing sweep rate reflects the charge transport kinetics in the material. The peak shift of our $\mathrm{TiO}_{2}-\mathrm{B}$ thin films is similar to, if not smaller than, literature reports of $\mathrm{TiO}_{2}-\mathrm{B}$ electrodes where the $\mathrm{CV}$ curves were also taken at the same scan rates. ${ }^{24}$

An important factor to consider is that for the highly crystalline films studied here which are 50-100 $\mathrm{nm}$ thick, $\mathrm{Li}^{+}$exchange and current collection occur only at the surface, so the $\mathrm{Li}$ transport path in battery cycling is much longer than that of many high efficiency $\mathrm{TiO}_{2}-\mathrm{B}$ nanostructures (usually powders heavily mixed with carbon black as current collector) in the reports, for instance, $6 \mathrm{~nm}$ average grain size in mesoporous microsphere, ${ }^{23}$ or 5-10 nm thickness in nanosheets. ${ }^{24}$ The fact that our films showed comparable charge storage kinetics is likely a result of the water free, high quality structures.

Each CV loop is comprised of two CV curves sweeping from 1 to $3 \mathrm{~V}$ and from 3 to $1 \mathrm{~V}$. During every sweep of the voltage, the electrical power exerted by the battery can be calculated by integrating the $\mathrm{CV}$ curve, and an average current can be deduced:

$$
\begin{gathered}
P_{\text {integ }}=\int i \mathrm{~d} E \\
\bar{\imath}=\frac{P_{\text {integ }}}{\Delta E}
\end{gathered}
$$

where $\Delta E$ is the range of the voltage window, or $2 \mathrm{~V}$ in this case. Combining (4.5) and (4.7), the pseudocapacitance, which represents the charge capacity of the pseudocapacitive material, can be calculated as:

$$
C=\frac{\bar{\imath}}{v}
$$

We have calculated the specific capacitance by integrating the CVs and found the capacitance is almost the same from either the oxidation or the reduction curve, regardless of scan rate, which also corresponds well with the specific capacity obtained from galvanostatic cycling, as shown in Figure 4.15(b). Such result offers additional proof that the $\mathrm{TiO}_{2}$-B film, though thin, was actually being lithiated during cycling, because if the electrical charge was only 
stored on the surface, the capacitance would vary at different scan rates as in a capacitor.

\subsection{Structural stability of the $\mathrm{Ca}: \mathrm{TiO}_{2}-\mathrm{B}$ and $\mathrm{TiO}_{2}-\mathrm{B}$ films}

The materials' response to the intensive cycling was examined by XRD and TEM. XRD patterns of both the $\mathrm{TiO}_{2}-\mathrm{B}$ and $\mathrm{Ca}: \mathrm{TiO}_{2}-\mathrm{B}$ structures before and after being cycled for over 40 days are essentially unchanged (Figure 4.16). Atomic resolution TEM analysis confirmed that all the bronze structures stayed intact without any signs of significant degradation or collapse into other $\mathrm{TiO}_{2}$ polymorphs. The film surfaces were also clean, without any observation of SEI layer formation or Li plating.

Relatively subtle changes, however, were observed by comparing the images before and after cycling in these horizontally oriented (001) films. Figure 4.17(b) shows a region near the interface between $\mathrm{TiO}_{2}-\mathrm{B}$ and $\mathrm{Ca}: \mathrm{TiO}_{2}-\mathrm{B}$ template in an as-grown dual layer film on (100) $\mathrm{SrTiO}_{3}$. A straight, inclined anatase "wall" $\sim 3 \mathrm{~nm}$ wide stems from the interface and extends to the surface, separating two $\mathrm{TiO}_{2}-\mathrm{B}$ grains. The interface is formed between the $\mathrm{TiO}_{2}-\mathrm{B}(\overline{2} 01)$ plane and the anatase (103) plane. $^{6}$ Upon lithiation, the anatase phase experiences a volumetric increase along the wall, while the $\mathrm{TiO}_{2}-\mathrm{B}$ grain undergoes a contraction along its $c$-axis, ${ }^{121,122}$ and consequently creates a small fracture at the interface, as shown by the HAADF image in Figure 4.17(b). Such fractures were seen repeatedly in the post-cycling sample along the anatase walls. Figure 4.18 shows a wider area of the same film, where such fracturing always occurs along the inclined anatase walls throughout the film, some at the interface of $\mathrm{TiO}_{2}-\mathrm{B}$ and $\mathrm{Ca}: \mathrm{TiO}_{2}-\mathrm{B}$ and others inside the $\mathrm{TiO}_{2}-\mathrm{B}$ phase. Therefore, this should be an actual effect of the

$\mathrm{Li}^{+}$insertion and extraction rather than an incidental event. A longer fracture could form between two parallel anatase walls. Because of the layered structure of $\mathrm{TiO}_{2}-\mathrm{B}$, all fractures are parallel to the $a-b$ planes.

To argue the fractures are actually caused by volume expansion of anatase upon lithiation, which is $\sim 4 \%,{ }^{27}$ while the thickness of anatase is only $3 \mathrm{~nm}$, requires a process of elimination, explained as follows: 
(1) These fractures were not observed in as-grown films, but only appeared after cycling throughout the film, as seen in Figure 4.18. So this must be an effect of the cycling.

(2) This effect must be related to a certain mechanic force that is not uniform for different phases in the film.

(3) The fractures occur not only at the interface of $\mathrm{Ca}: \mathrm{TiO}_{2}-\mathrm{B}$ and $\mathrm{TiO}_{2}-\mathrm{B}$ layers, but in the $\mathrm{TiO}_{2}$-B phase itself as well. So this is not due to the inhomogeneity between these two phases.

(4) The fractures are always touching the anatase phase. They do not exist in the $\mathrm{Ca}: \mathrm{TiO}_{2}-\mathrm{B}$ layer where there are no such inclined anatase walls. Therefore it is really between the anatase phase and the $\mathrm{TiO}_{2}-\mathrm{B}$ phase.

(5) Both the anatase and $\mathrm{TiO}_{2}-\mathrm{B}$ phases are quite insulating, so it is unlikely to be induced by current flow, or a dielectric/piezoelectric effect at room temperature.

(6) We have monitored the cell temperature during cycling, which was not obviously higher than room temperature. So this is not an effect of heating/thermal expansion.

In light of the above analysis, volume expansion due to $\mathrm{Li}$ intercalation is the most likely cause for the fractures, which is also supported by the literature reports. The anatase phase in Figure 4.17(b) is extending on its $b$-axis, which experiences an increase along the wall upon lithiation. ${ }^{27}$ In contrast, $\mathrm{TiO}_{2}-\mathrm{B}$ is grown along its $c$-axis, which undergoes a contraction. ${ }^{121}$ In this 1D system along the out-of-plane direction, this anatase wall, though thin, may still result in a significant amount of stress load at its phase boundary with $\mathrm{TiO}_{2}-\mathrm{B}$, especially at one tip end, and hence the peeling type of shearing in Figure 4.17(b).

In an effort to confirm that $\mathrm{Li}^{+}$was actually inserted into the bronze films during cycling, fine XRD scans were performed on a (001) $\mathrm{Ca}_{2} \mathrm{TiO}_{2}-\mathrm{B}$ film grown on a (100) $\mathrm{SrTiO}_{3}$ substrate around the strongest available diffraction peak, 006, before and after charging with $\mathrm{Li}^{+}$at a rate of $\mathrm{C} / 10$. The results are shown in Figure 4.19(a). Using the $\mathrm{SrTiO}_{3}$ substrate peaks as reference, the 006 peak of the lithiated film has shifted to lower $2 \theta$ angles, indicating a lattice expansion in the out-of-plane direction from $c_{\text {Ca:TiO2-B }}=17.98 \AA$ to $18.04 \AA$. By fitting the experimental data, it 
is also clear that the peak has broadened as the inserted $\mathrm{Li}^{+}$disturbs the crystallinity of the lattice structure. The broadening is mainly caused by local straining within the thin film as the unit cell undergoes an asymmetric deformation upon lithiation. Structural defects such as dislocations and stacking faults may also contribute to straining effects in close regions and thus to the peak broadening. The fractures observed in the cycled films (Figure 4.18) contribute to the broadening as well, as some planes are pushed slightly out-of-place by the cracks. If the crack is large enough, it essentially reduces the grain size and results in an XRD peak broadening.

The same experiments were performed around the $\mathrm{TiO}_{2}-\mathrm{B} 001$ peak of a (001) $\mathrm{TiO}_{2}-\mathrm{B} / \mathrm{Ca}: \mathrm{TiO}_{2}-\mathrm{B}$ dual layer film grown on a (100) $\mathrm{SrTiO}_{3}$ substrate. Figure 4.19(b) shows that $c_{\text {TiO2-B }}$ exhibits a slight contraction of $\sim 0.21 \%$ upon lithiation, which is in good agreement with the literature, ${ }^{121}$ where neutron diffraction results revealed a contraction in $c_{\text {TiO2-B }}$ of $0.18 \%$ $0.49 \%$, depending on the Li content. An obvious peak broadening was again observed. Changes in the other lattice constants were not examined.

It should be noted that the lattice constant changes we observed with this post-mortem procedure may not correspond to fully lithiated films. The thin films have a large surface and a small mass, so an unknown amount of Li charged into the film could be lost to more stable lithium oxides forming on the surface when the sample was exposed to air and the electrode was examined. ${ }^{123}$ Although this might also happen in the more typical powder samples of $\mathrm{TiO}_{2}-\mathrm{B}$, the much larger amount of active material used there may ensure that more of the $\mathrm{Li}$ is retained in the sample. Such a difference in sample geometry may help explain the discrepancy between our observation and the reported values in the literature. Water and anatase impurities may also have an effect on the values reported for $\mathrm{TiO}_{2}-\mathrm{B}$ in the literature.

\subsection{Summary and conclusions}

In summary, high quality (001) $\mathrm{Ca}_{\mathrm{TiO}}-\mathrm{B}$ and $(001) \mathrm{TiO}_{2}-\mathrm{B}$ (with a thin $\mathrm{Ca}: \mathrm{TiO} 2-\mathrm{B}$ template) films were investigated as anode materials for lithium-ion batteries, using a specially designed testing configuration and data acquisition/processing protocol. The following 
conclusions have been reached:

(1) The top current collection configuration with a $\mathrm{Cu}$ wire grid and the bottom current collection configuration with a conductive $\mathrm{Nb}: \mathrm{SrTiO}_{3}$ substrate generated similar and proven valid test results, with the former performing better at higher charge/discharge rates.

(2) Both $\mathrm{Ca}: \mathrm{TiO}_{2}-\mathrm{B}$ and $\mathrm{TiO}_{2}-\mathrm{B}$ exhibit $\mathrm{Li}$ electrochemical reactivity, with a pseudocapacitive intercalation behavior where the voltage profiles are sloped. No well-developed plateau is observed in the voltage profiles.

(3) $\mathrm{Ca}_{\mathrm{TiO}_{2}}-\mathrm{B}$ has a theoretical capacity of $294 \mathrm{~mA} \mathrm{~h} \mathrm{~g}$, and experimentally discharges to $273 \mathrm{~mA} \mathrm{~h} \mathrm{~g}^{-1}$, agreeing with theoretical calculations that $18 \mathrm{Li}$ sites may be filled during intercalation in a $\mathrm{Ca}_{4} \mathrm{Ti}_{20} \mathrm{O}_{44}$ unit cell.

(4) $\mathrm{TiO}_{2}-\mathrm{B}$ has a theoretical capacity of $335 \mathrm{~mA} \mathrm{~h} \mathrm{~g}^{-1}$, and experimentally discharges to $334 \mathrm{~mA} \mathrm{~h} \mathrm{~g}^{-1}$ at a rate of $\mathrm{C} / 10$. A pair of redox peaks is observed on the $\mathrm{CV}$ curvs at $1.54 \mathrm{~V}$ and $1.69 \mathrm{~V}$. The specific capacitance calculated by integrating the CVs is independent of the voltage scan rate, confirming that the thin film is actually being lithiated during battery cycling, and that the contribution to charge capacity is Faradaic, referred to as a 'pseudocapacitive Faradaic process' in the literature. ${ }^{16}$

(5) Capacity retention is good for both $\mathrm{Ca}: \mathrm{TiO}_{2}-\mathrm{B}$ and $\mathrm{TiO}_{2}-\mathrm{B}$, with major loss resulting from the poor electrical conductivity of the materials.

(6) Structural stability is excellent for both $\mathrm{Ca}: \mathrm{TiO}_{2}-\mathrm{B}$ and $\mathrm{TiO}_{2}-\mathrm{B}$, without any signs of phase transformation in the films, or SEI layer formation or Li plating on the surface. Small fractures are observed in post-cycling $\mathrm{TiO}_{2}-\mathrm{B}$ films caused by thin layers of anatase defects.

(7) The bronze structures undergo small deformation during Li intercalation: a slight increase in the $c$-axis lattice constant of $\mathrm{Ca}: \mathrm{TiO}_{2}-\mathrm{B}$ and a slight decrease in that of $\mathrm{TiO}_{2}-\mathrm{B}$. A broadening of the XRD peaks is also observed in both structures as the inserted $\mathrm{Li}^{+}$causes local straining. Limited by the thin film geometry, changes in the other lattice constants were not examined for this study. 
Table 4.1 List of a few popular cathode materials.

\begin{tabular}{|c|c|c|c|c|}
\hline Material & $\begin{array}{c}\text { Capacity }\left(\mathrm{mA} \mathrm{h} \mathrm{g}^{-1}\right) \\
\text { Theoretical/Experimental }\end{array}$ & $\begin{array}{c}\text { Potential } \\
\left(\text { V vs. } \mathrm{Li} / \mathrm{Li}^{+}\right)\end{array}$ & Notes & Ref. \\
\hline $\mathrm{LiCoO}_{2}$-layered & $273 / 160$ & 3.9 & $\begin{array}{c}\text { Good rate capability, } \\
\text { stable cycling properties; } \\
\text { toxic, expensive }\end{array}$ & [91] \\
\hline $\mathrm{Li}\left(\mathrm{Co}_{x} \mathrm{Ni}_{y} \mathrm{Mn}_{z}\right) \mathrm{O}_{2}$ & $\sim 270 / 150-180$ & $\sim 3.8$ & Cheaper, stable & $\begin{array}{l}{[94,95,} \\
101-105]\end{array}$ \\
\hline $\mathrm{LiMn}_{2} \mathrm{O}_{4}$-spinel & $148 / 130$ & 4.1 & $\begin{array}{c}\text { Cheap, green; lower } \\
\text { capacity }\end{array}$ & [124] \\
\hline $\mathrm{LiFePO}_{4}$-olivine & $170 / 166$ & 3.45 & $\begin{array}{l}\text { Cheap, green, ultrafast } \\
\text { charging/discharging }\end{array}$ & $\begin{array}{l}{[15,125,} \\
126]\end{array}$ \\
\hline
\end{tabular}


Table 4.2 List of a few popular anode materials.

\begin{tabular}{|c|c|c|c|c|}
\hline Material & $\begin{array}{l}\text { Capacity }\left(\mathrm{mA} \mathrm{h}^{-1}\right) \\
\text { Theoretical/Experimental }\end{array}$ & $\begin{array}{c}\text { Potential } \\
\left(\mathrm{V} \text { vs. } \mathrm{Li} / \mathrm{Li}^{+}\right)\end{array}$ & Notes & Ref. \\
\hline Graphite & $372 / 370$ & $0.1-0.2$ & $\begin{array}{l}\mathrm{LiC}_{6}, \text { excellent stability, } \\
\text { volume change } \sim 11 \%\end{array}$ & [127] \\
\hline Si (composite) & $4200 / \sim 1000-2500$ & $<0.5$ & $\begin{array}{l}\text { Severe volume changes } \\
(>300 \%) \text {, poor cycling }\end{array}$ & $\begin{array}{l}{[128,129,} \\
130]\end{array}$ \\
\hline $\mathrm{Li}_{4} \mathrm{Ti}_{5} \mathrm{O}_{12}$-spinel & $175 / 170$ & 1.55 & $\begin{array}{c}\text { High voltage, negligible } \\
\text { volume change }\end{array}$ & {$[32,131]$} \\
\hline $\begin{array}{l}\mathrm{TiO}_{2} \\
\text { (anatas/rutile) }\end{array}$ & $335 / 100-250$ & $1.7-2.1$ & $\begin{array}{l}\text { Cheap, small volume } \\
\text { change }(\sim 4 \%)\end{array}$ & {$[29,30]$} \\
\hline $\mathrm{TiO}_{2}-\mathrm{B}$ & $335 / 334$ & 1.6 & $\begin{array}{l}\text { Open structure, high rate } \\
\text { capability }\end{array}$ & This work \\
\hline $\begin{array}{l}\mathrm{Ca}: \mathrm{TiO}_{2}-\mathrm{B} \\
\left(\mathrm{CaTi}_{5} \mathrm{O}_{11}\right)\end{array}$ & $294 / 293$ & 1.5 & $\begin{array}{l}\text { Superfast charging with } \\
\text { orientation engineering }\end{array}$ & This work \\
\hline
\end{tabular}




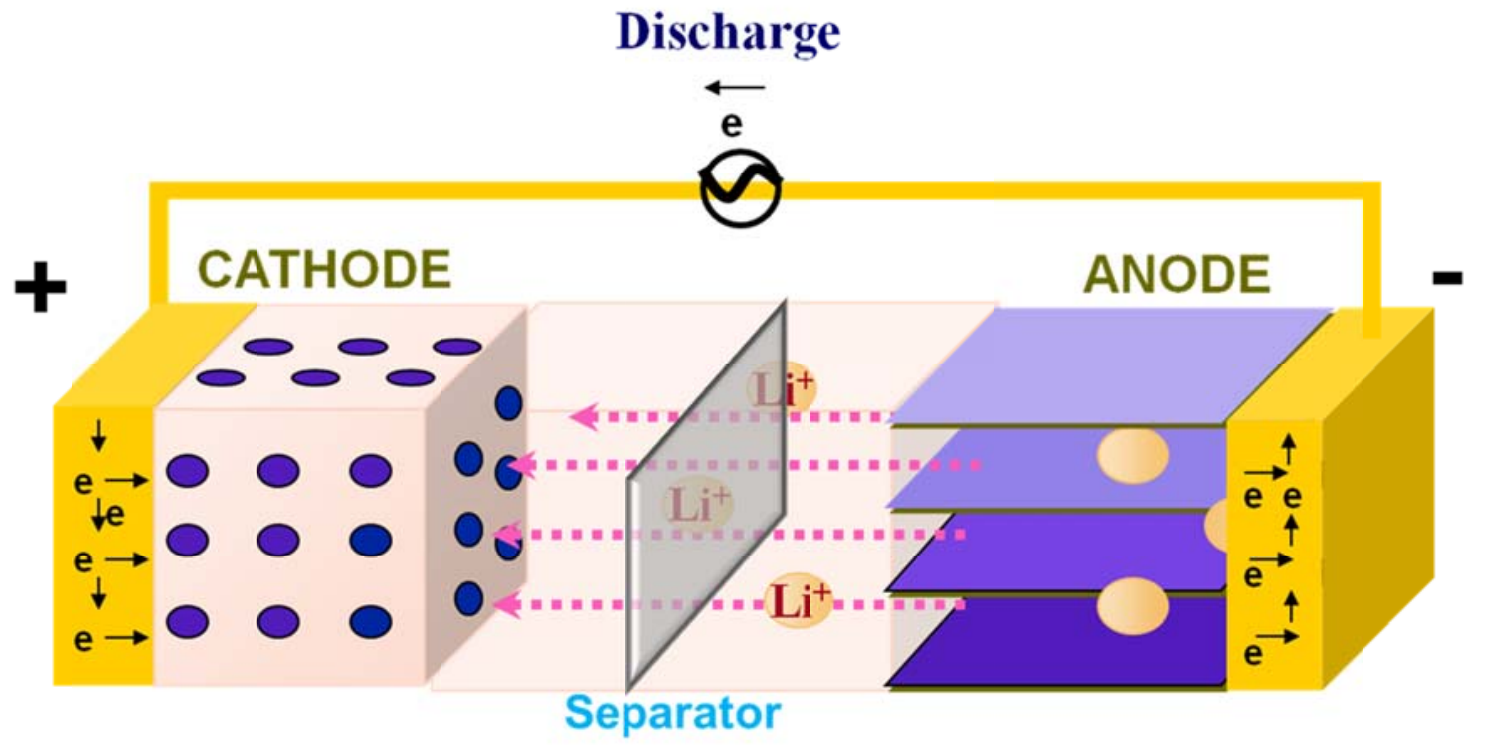

Figure 4.1 Schematic of a typical lithium-ion battery cell. 


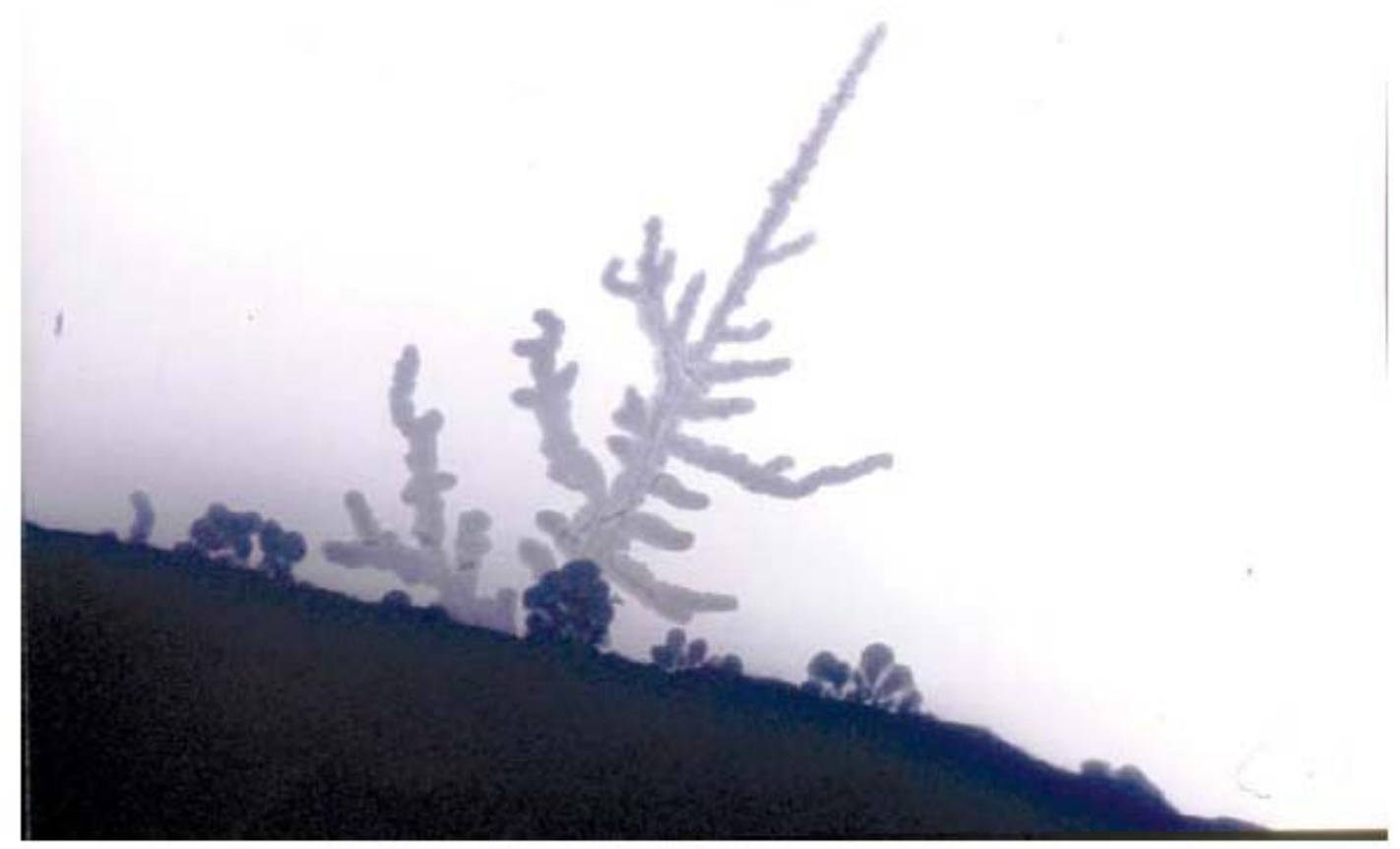

Figure 4.2 Dendritic growth of lithium during its electro-deposition. [From Ref. 132] 


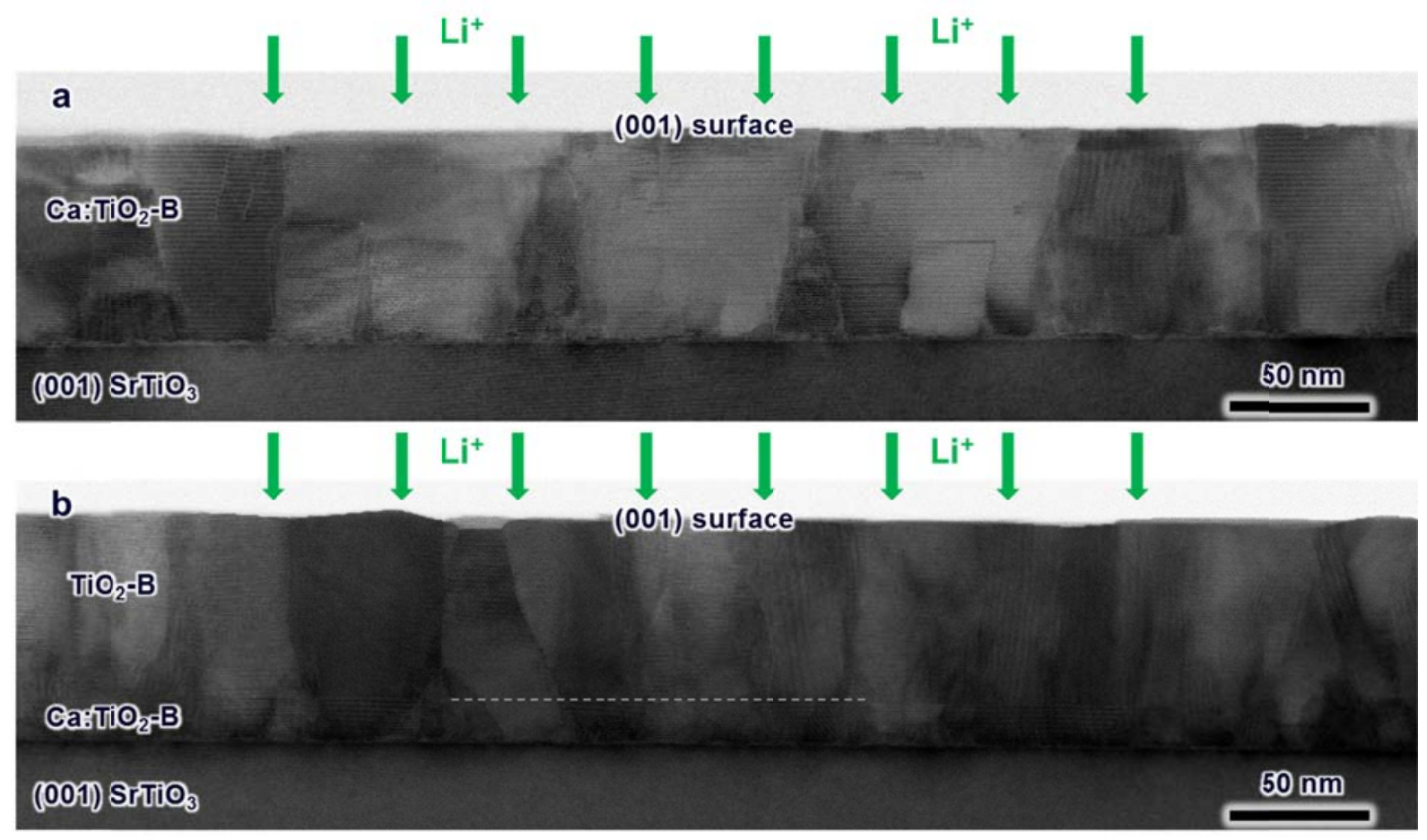

Figure 4.3 As-grown morphology of both the (001) $\mathrm{Ca}: \mathrm{TiO}_{2}$ thin film and the $(001)$ $\mathrm{TiO}_{2}-\mathrm{B} / \mathrm{Ca}: \mathrm{TiO}_{2}-\mathrm{B}$ dual layer film on (100) $\mathrm{SrTiO}_{3}$ substrate. (a) Low magnification STEM image of a $\mathrm{Ca}: \mathrm{TiO}_{2}-\mathrm{B}$ film with $\sim 75 \mathrm{~nm}$ thickness. (b) Low magnification STEM image of a $\mathrm{TiO}_{2}-\mathrm{B}$ film $(\sim 65 \mathrm{~nm})$ grown on top of a thin $\mathrm{Ca}: \mathrm{TiO}_{2}-\mathrm{B}$ template layer $(\sim 15 \mathrm{~nm})$. The dashed line was drawn to locate the interface. [TEM image credit: Michael B. Katz] 

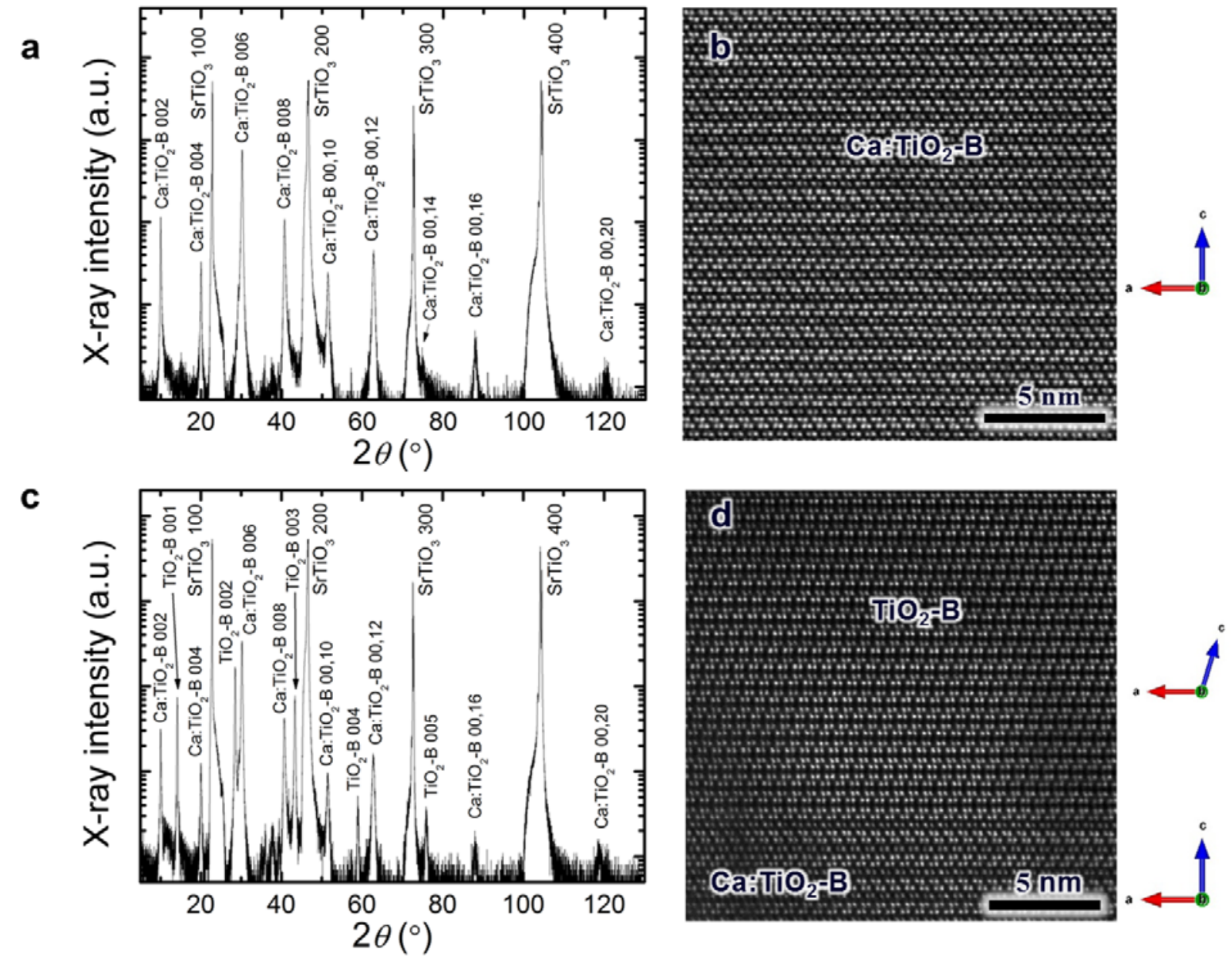

Figure 4.4 Structural characterization of $\mathrm{Ca}: \mathrm{TiO}_{2}-\mathrm{B}$ and regular $\mathrm{TiO}_{2}-\mathrm{B}$. (a) XRD pattern of a (001) $\mathrm{Ca}_{\mathrm{TiO}}-\mathrm{B}$ thin film grown on a (001) $\mathrm{SrTiO}_{3}$ substrate at $800{ }^{\circ} \mathrm{C}$ by PLD. The hetero-epitaxial relationship is [100](001) $)_{\text {Ca:TiO2-B }} \|$ [100](001) $)_{\text {SrTiO3. }}$ (b) HAADF STEM image of the $\mathrm{Ca}: \mathrm{TiO}_{2}$-B phase. (c) XRD pattern of a regular (001) $\mathrm{TiO}_{2}-\mathrm{B}$ thin film grown on top of a thin $\mathrm{Ca}: \mathrm{TiO}_{2}-\mathrm{B}$ template layer on a (001) $\mathrm{SrTiO}_{3}$ substrate under the same PLD conditions. (d) HAADF STEM image of the dual layer structure. [TEM image credit: Michael B. Katz] 


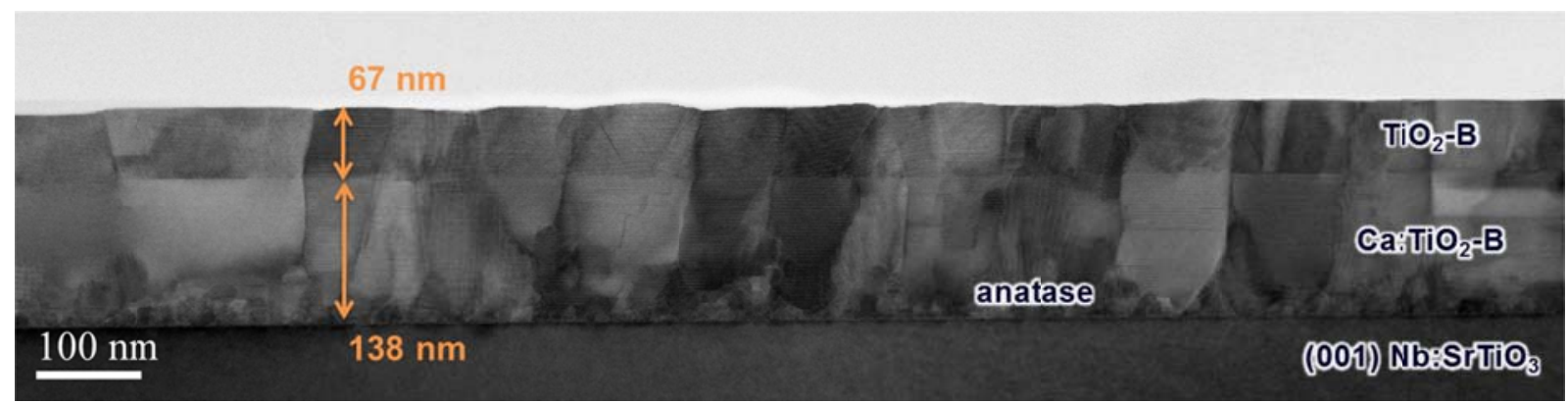

Figure 4.5 Bright field STEM image of a (001) $\mathrm{TiO}_{2}-\mathrm{B} / \mathrm{Ca}$ : $\mathrm{TiO}_{2}-\mathrm{B}$ dual layer film grown on a (001) $\mathrm{Nb}: \mathrm{SrTiO}_{3}$ substrate. Many small anatase grains formed at the interface. [TEM image credit: Michael B. Katz] 


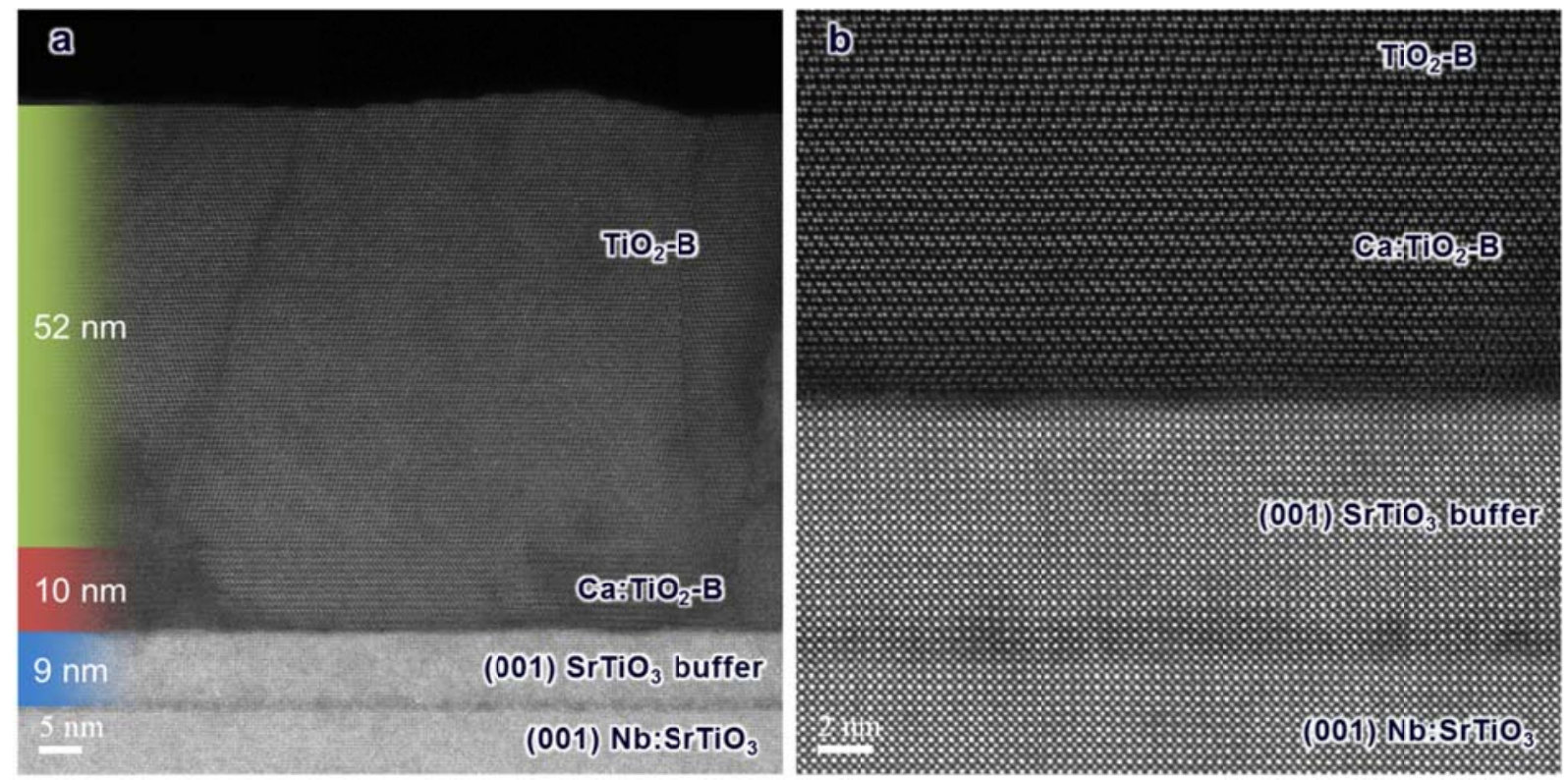

Figure 4.6 (a) Low magnification and (b) high magnification HAADF STEM images showing a (001) $\mathrm{TiO}_{2}-\mathrm{B} / \mathrm{Ca}: \mathrm{TiO}_{2}$-B dual layer film grown on a (001) $\mathrm{Nb}: \mathrm{SrTiO}_{3}$ substrate with a $\mathrm{SrTiO}_{3}$ buffer layer. [TEM image credit: Michael B. Katz] 

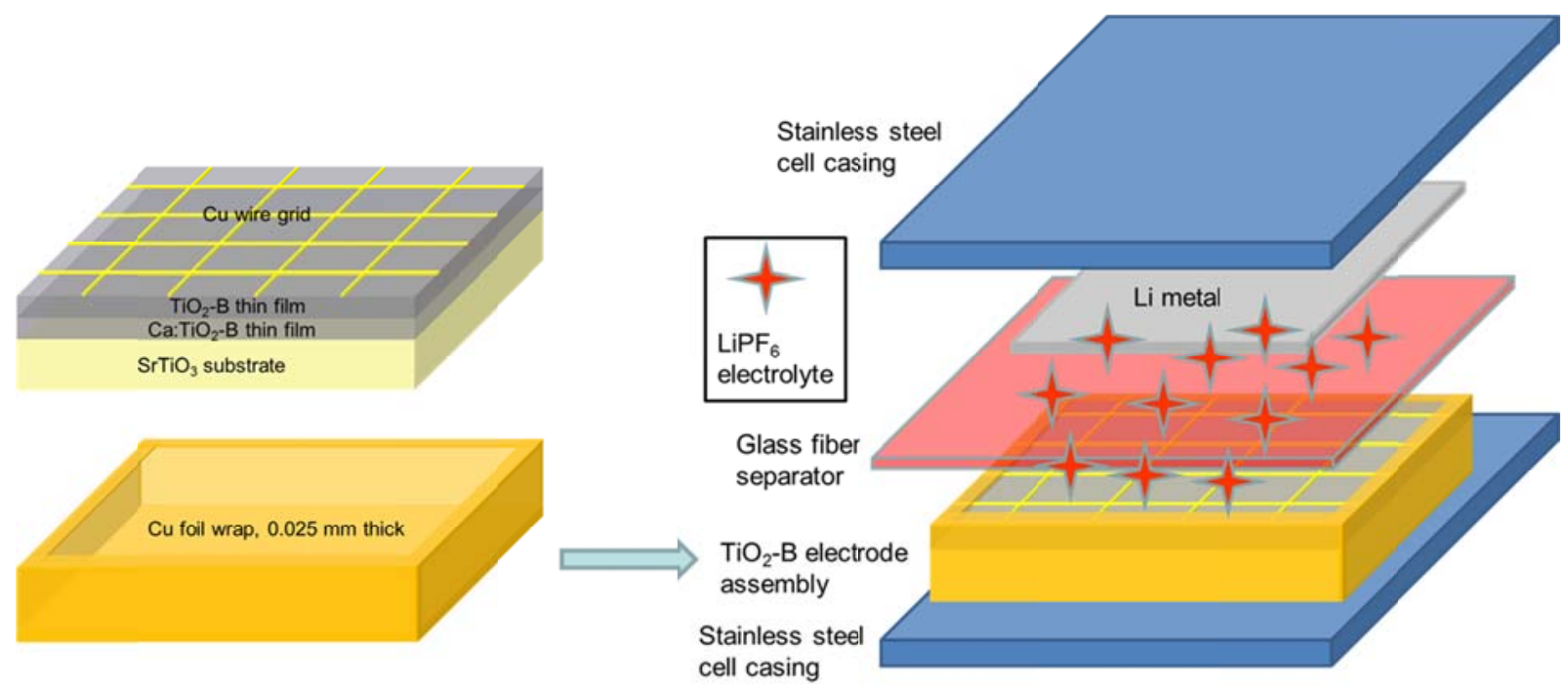

Figure 4.7 Top current collector configuration for electrochemical measurements. Most battery performance results reported in this thesis were acquired using such configuration from films grown on insulating $10 \times 10 \mathrm{~mm}^{2} \mathrm{SrTiO}_{3}$ substrates. 


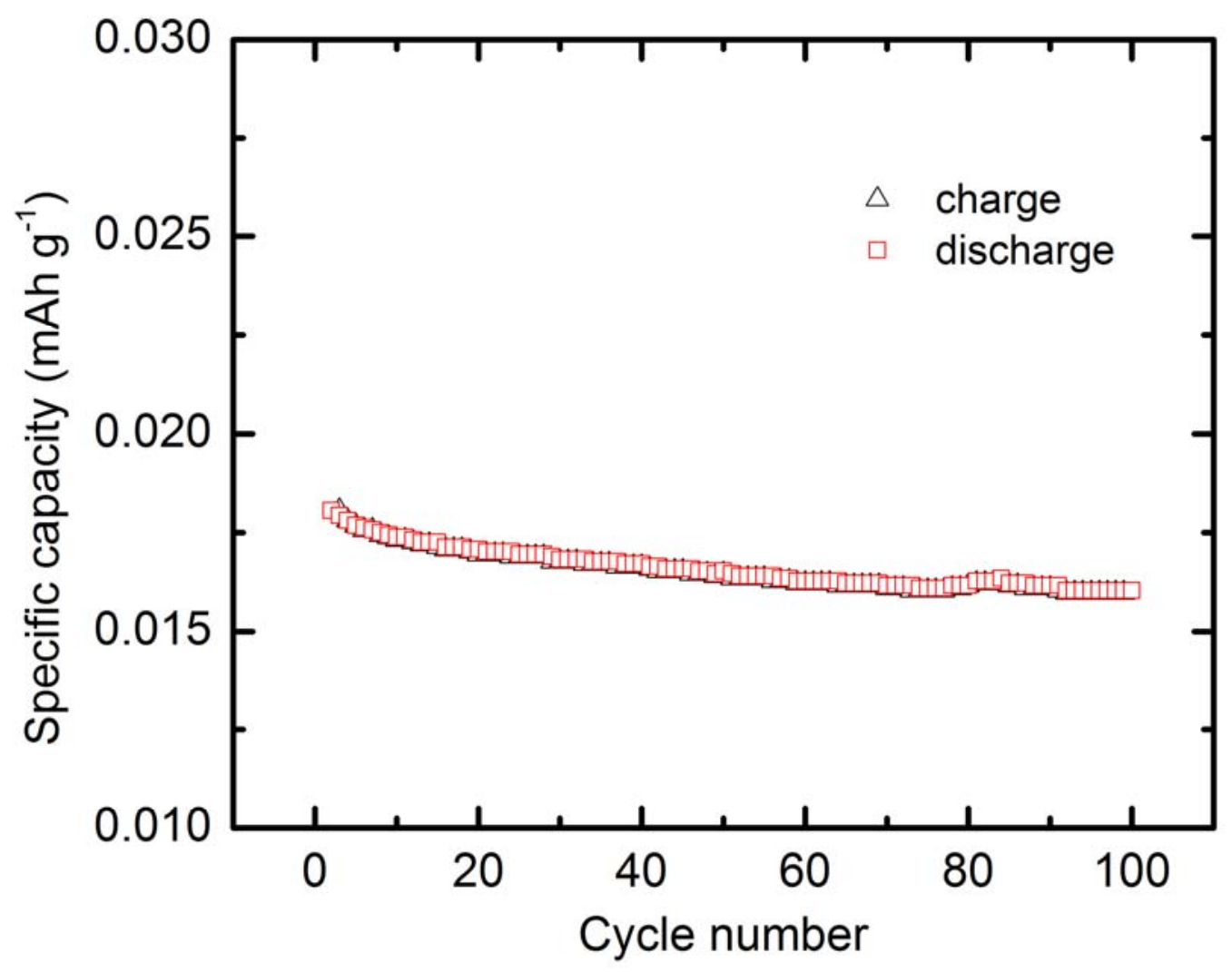

Figure 4.8 Cycling performance of a bare $\mathrm{Nb}: \mathrm{SrTiO}_{3}(001)$ substrate at a $1 \mathrm{C}$ rate for 100 cycles in a voltage window of 1-3 V versus Li metal. 


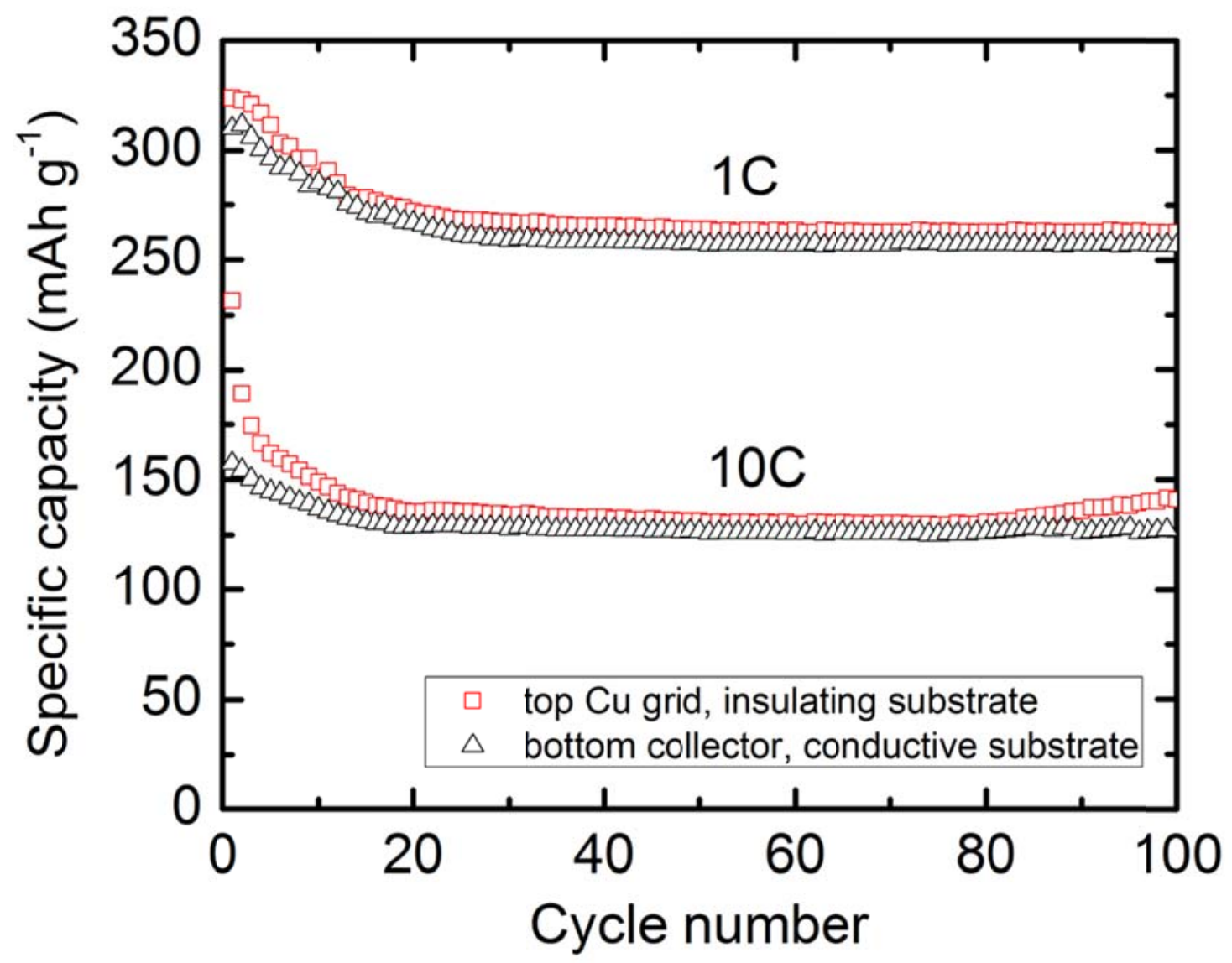

Figure 4.9 Comparison of discharge capacities of the $\mathrm{TiO}_{2}-\mathrm{B}$ thin film using two different test configurations. Capacity contribution from the $\mathrm{Ca}: \mathrm{TiO}_{2}-\mathrm{B}$ template layer was subtracted in both configurations; capacity of the conductive $\mathrm{Nb}: \mathrm{SrTiO}_{3}$ substrate was subtracted for the bottom collector configuration. 


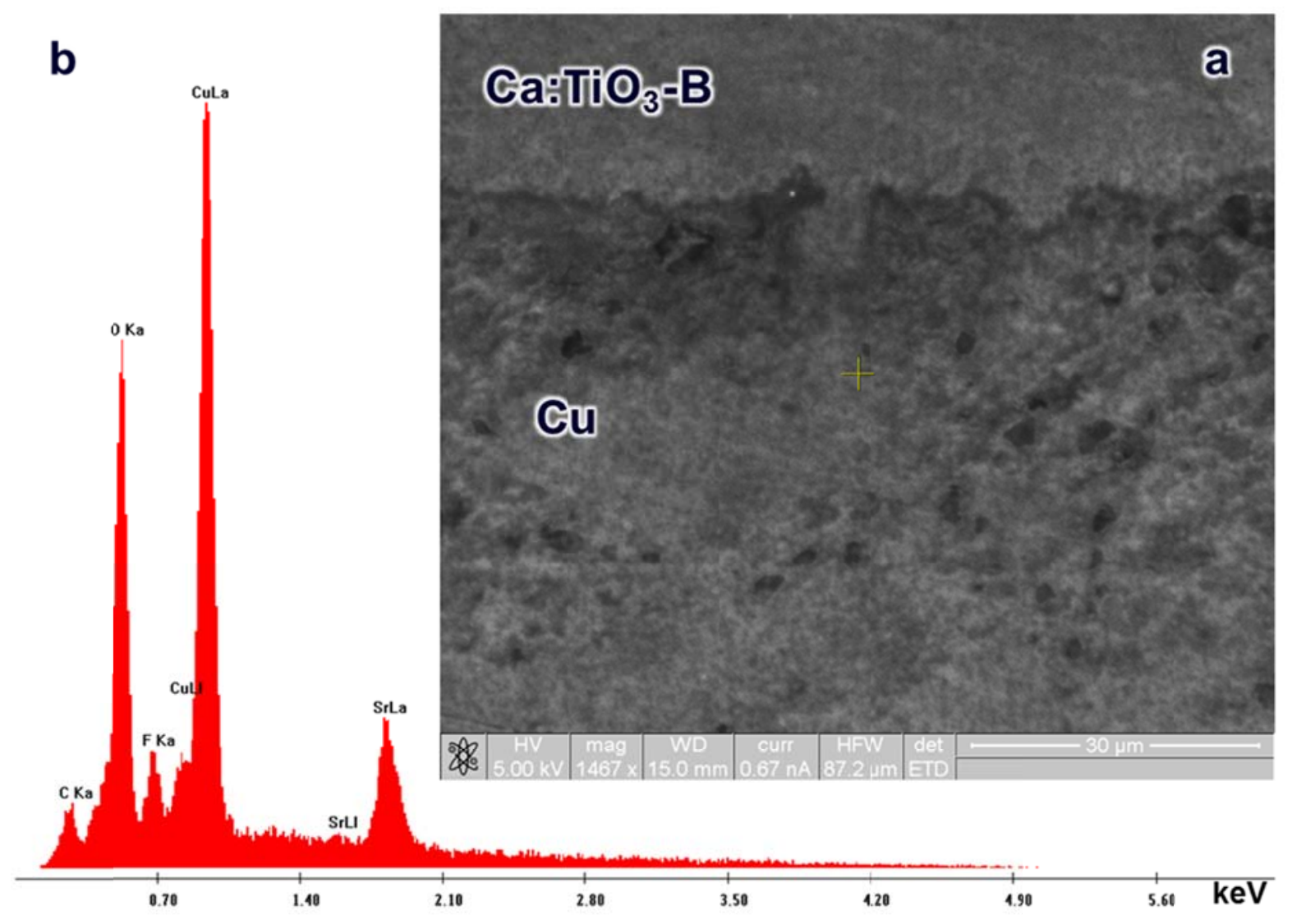

Figure 4.10 $\mathrm{Cu}$ grid surface at the charged state. (a) Scanning electron microscopy image showing the surface of the $\mathrm{Cu}$ wire on the $\mathrm{Ca}: \mathrm{TiO}_{2}-\mathrm{B}$ thin film. This film was fully charged with $\mathrm{Li}^{+}$at the rate of $1000 \mathrm{C}$, and removed from the cell at a half cycle to be examined. No obvious $\mathrm{Li}$ plating was observed. (b) X-ray energy dispersive spectrum taken on the $\mathrm{Cu}$ wire. Carbon and fluorine are from the residue of $\mathrm{LiPF}_{6}$ electrolyte. 


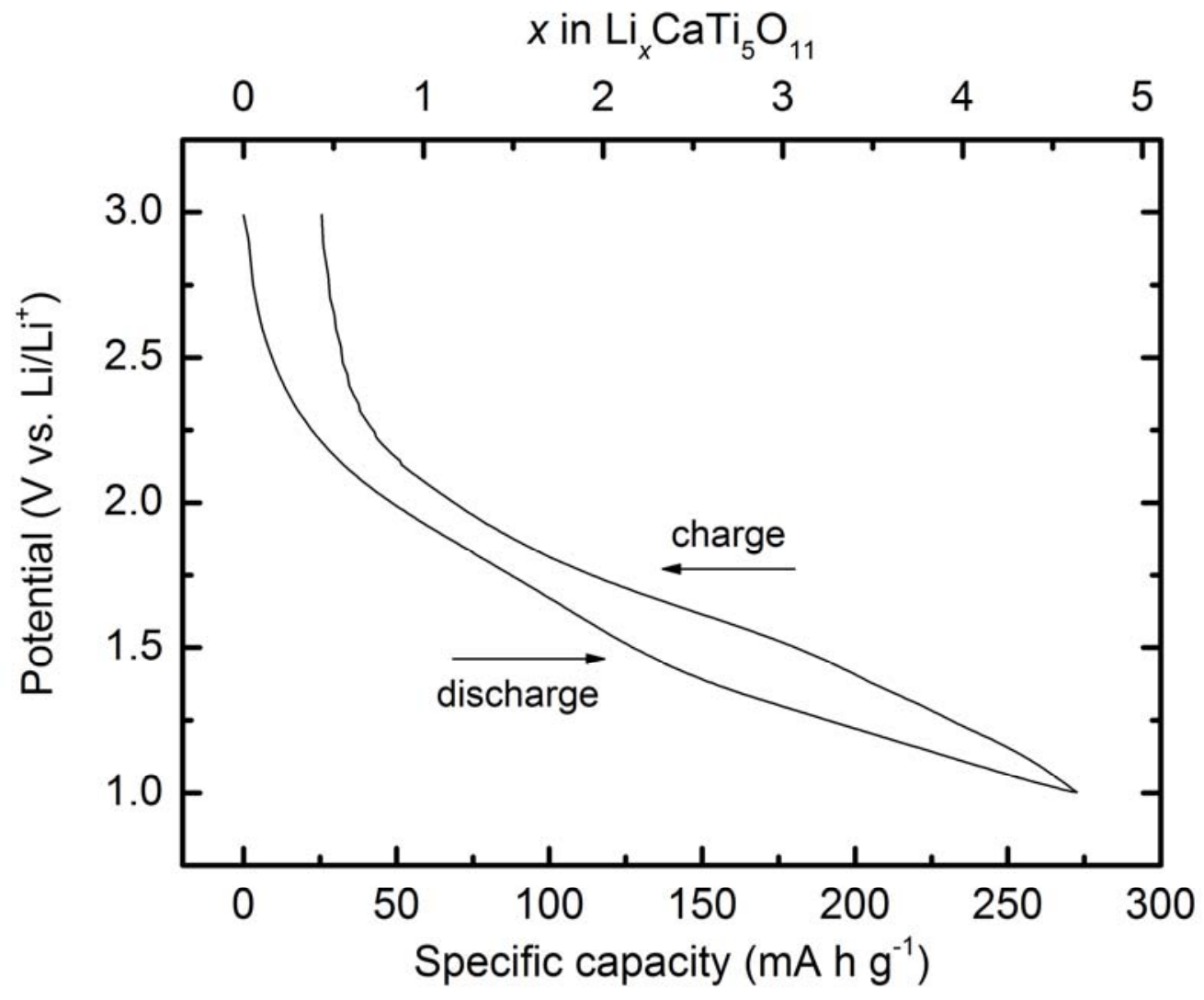

Figure 4.11 Voltage profile with $\mathrm{Li}$ content at the 8 th cycle of a $(001) \mathrm{Ca}: \mathrm{TiO}_{2}-\mathrm{B}$ thin film grown on a (001) $\mathrm{SrTiO}_{3}$ substrate versus Li metal at a rate of $33.5 \mathrm{~mA} \mathrm{~g}^{-1}$ between 1.0-3.0 V. 


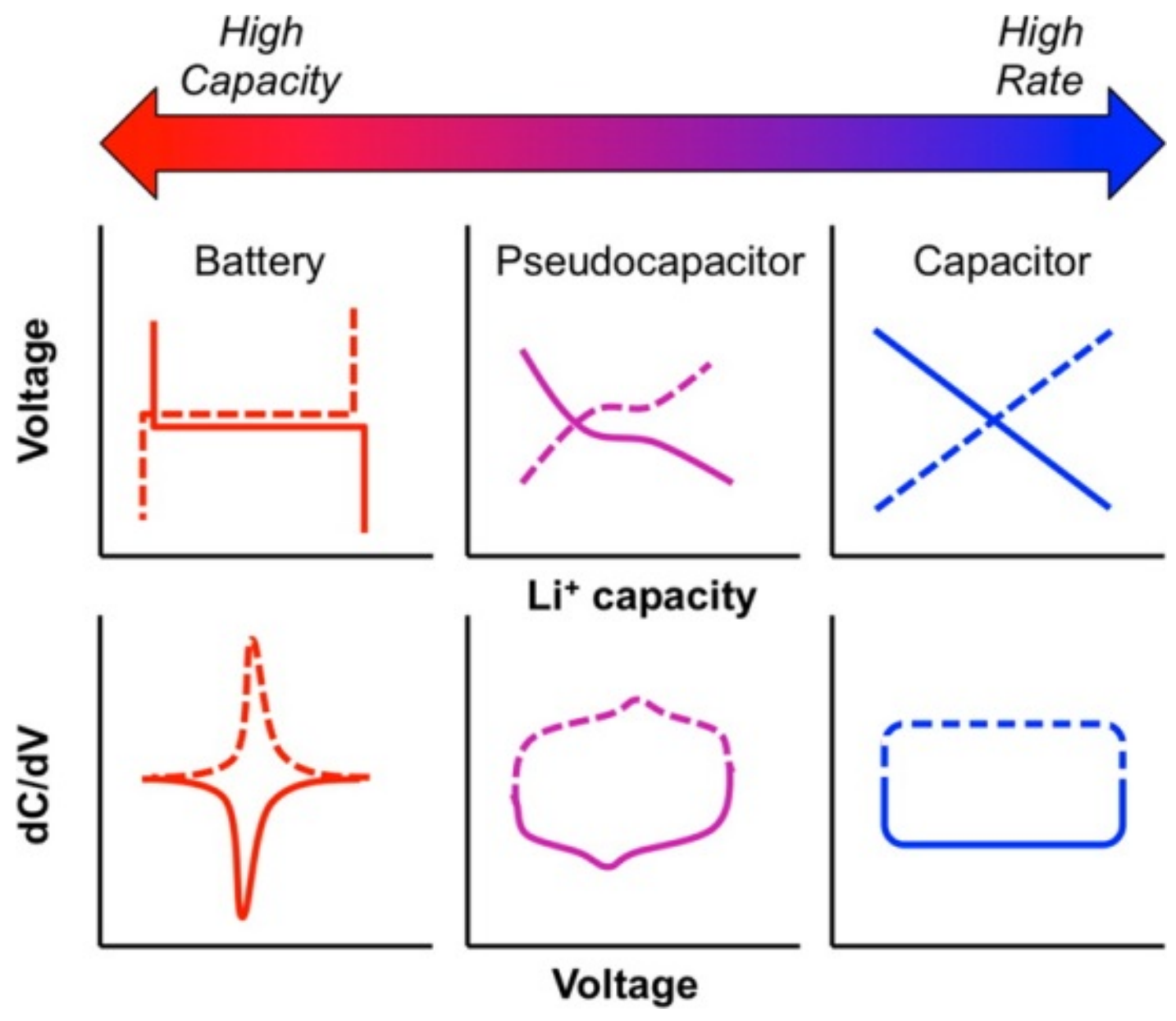

Figure 4.12 Schemes showing idealized voltage and differential capacity $(\mathrm{d} C / \mathrm{d} V)$ profiles for three basic charge storage mechanisms. [From Ref. 38] 


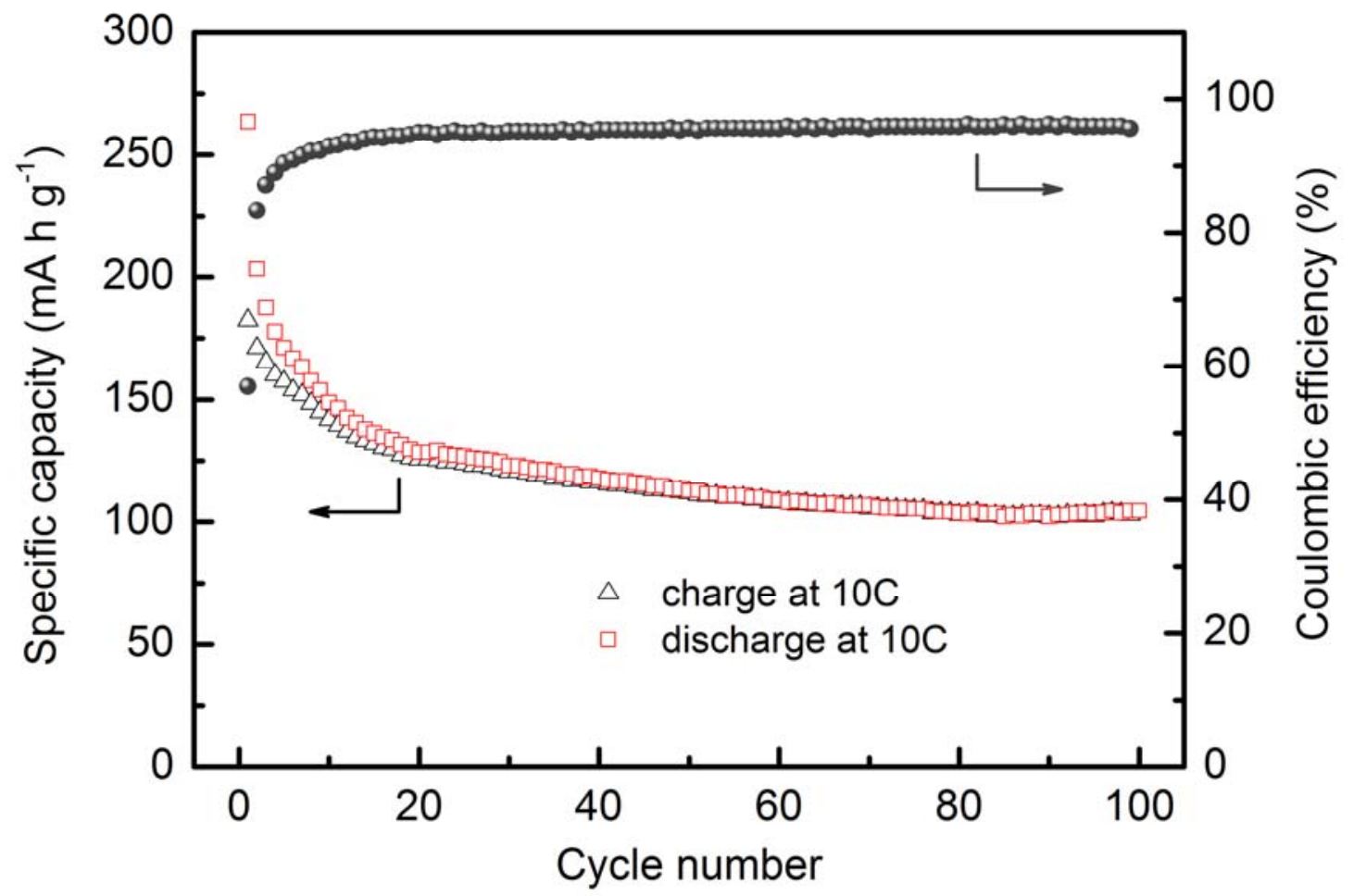

Figure 4.13 Cycling performance and Coulombic efficiency of a (001) $\mathrm{Ca}_{2} \mathrm{TiO}_{2}-\mathrm{B}$ thin film grown on a (001) $\mathrm{SrTiO}_{3}$ substrate at $10 \mathrm{C}\left(1 \mathrm{C}=294 \mathrm{~mA} \mathrm{~g}^{-1}\right)$ for 100 cycles. The voltage window is $1-3 \mathrm{~V}$. 

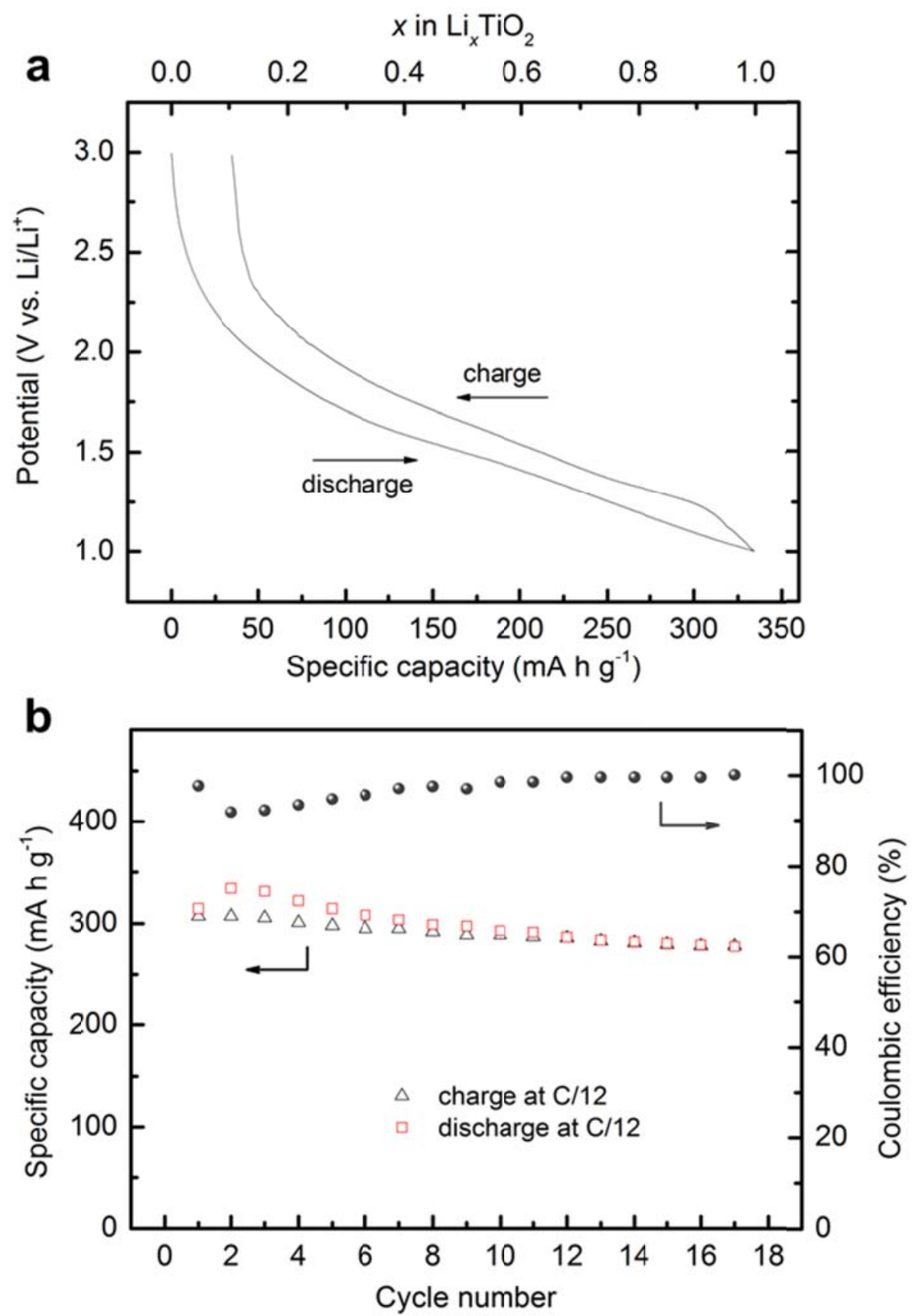

Figure 4.14 (a) Voltage profile with $\mathrm{Li}$ content at the 8th cycle of a (001) $\mathrm{TiO}_{2}-\mathrm{B}$ thin film grown with a $\mathrm{Ca}: \mathrm{TiO}_{2}-\mathrm{B}$ template layer on a (001) $\mathrm{SrTiO}_{3}$ substrate versus $\mathrm{Li}$ metal at a $\mathrm{C} / 10$ rate $\left(1 \mathrm{C}=335 \mathrm{~mA} \mathrm{~g}^{-1}\right)$ between $1.0-3.0 \mathrm{~V}$. (b) Slow rate $(\mathrm{C} / 12)$ cycling results for 18 cycles. 

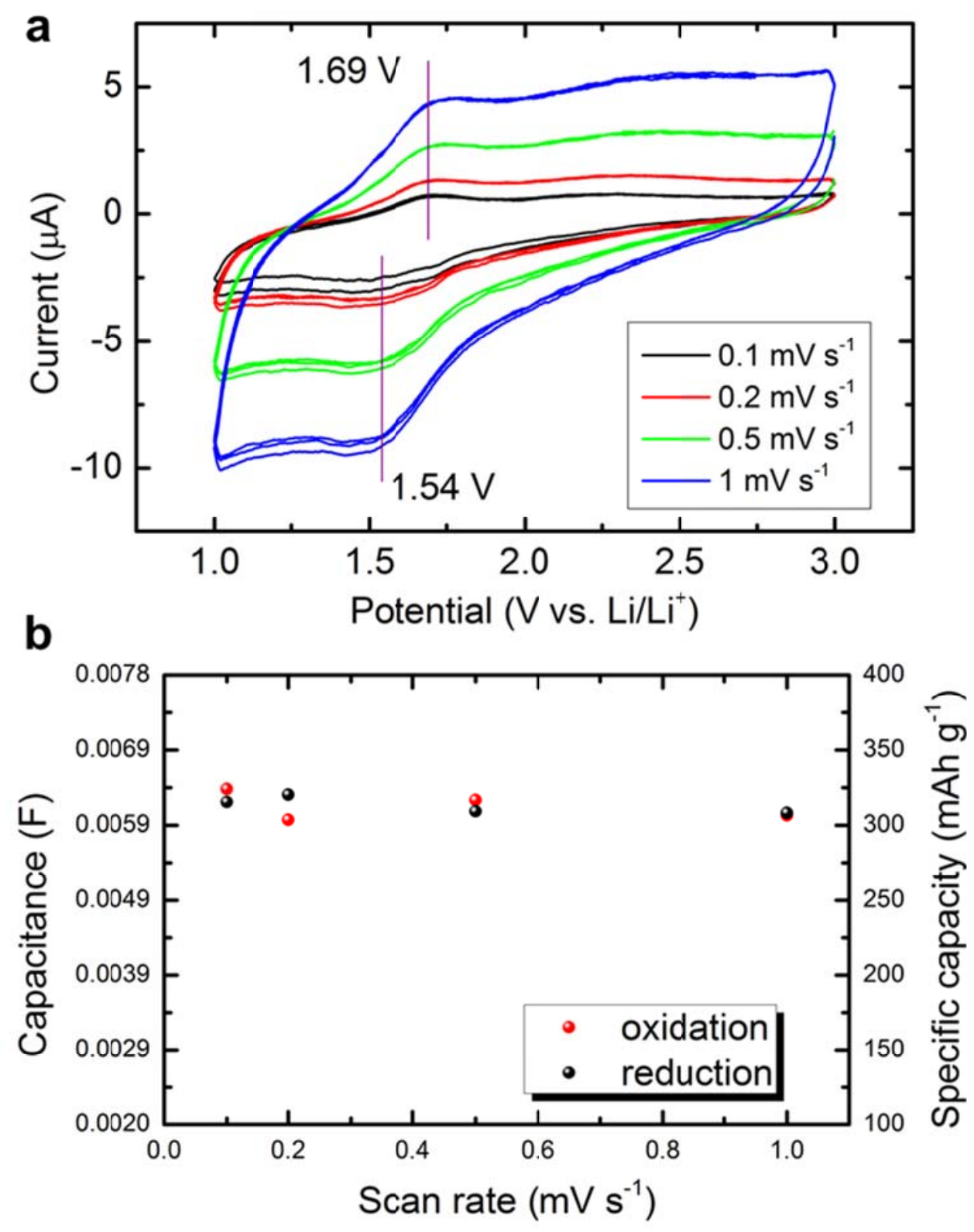

Figure 4.15 (a) Cyclic voltammograms (CVs) of a (001) $\mathrm{TiO}_{2}-\mathrm{B}$ thin film using the top $\mathrm{Cu}$ grid current collector at different scan rates. Three cycles are shown for each rate, demonstrating good repeatability of the measurement. Signature $\mathrm{TiO}_{2}-\mathrm{B}$ peaks were observed. (b) Specific capacitance calculated by integrating the CVs at different scan rates for both the oxidation and reduction curves. 
a
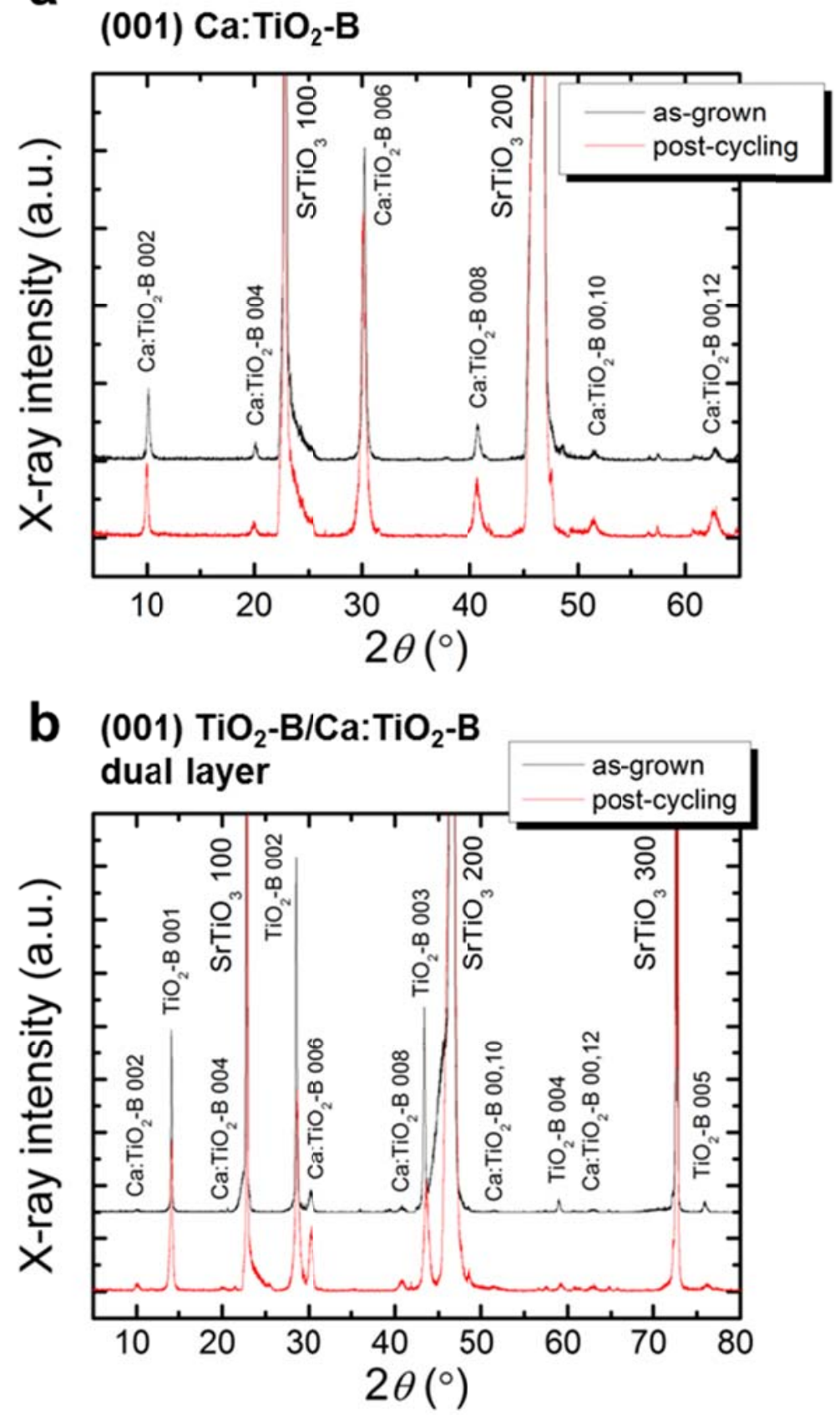

Figure 4.16 XRD $\theta-2 \theta$ patterns before and after cycling of (a) a (001) $\mathrm{Ca}: \mathrm{TiO}_{2}-\mathrm{B}$ film and (b) a (001) $\mathrm{TiO}_{2}-\mathrm{B} / \mathrm{Ca}: \mathrm{TiO}_{2}-\mathrm{B}$ dual layer film on (001) $\mathrm{SrTiO}_{3}$. 

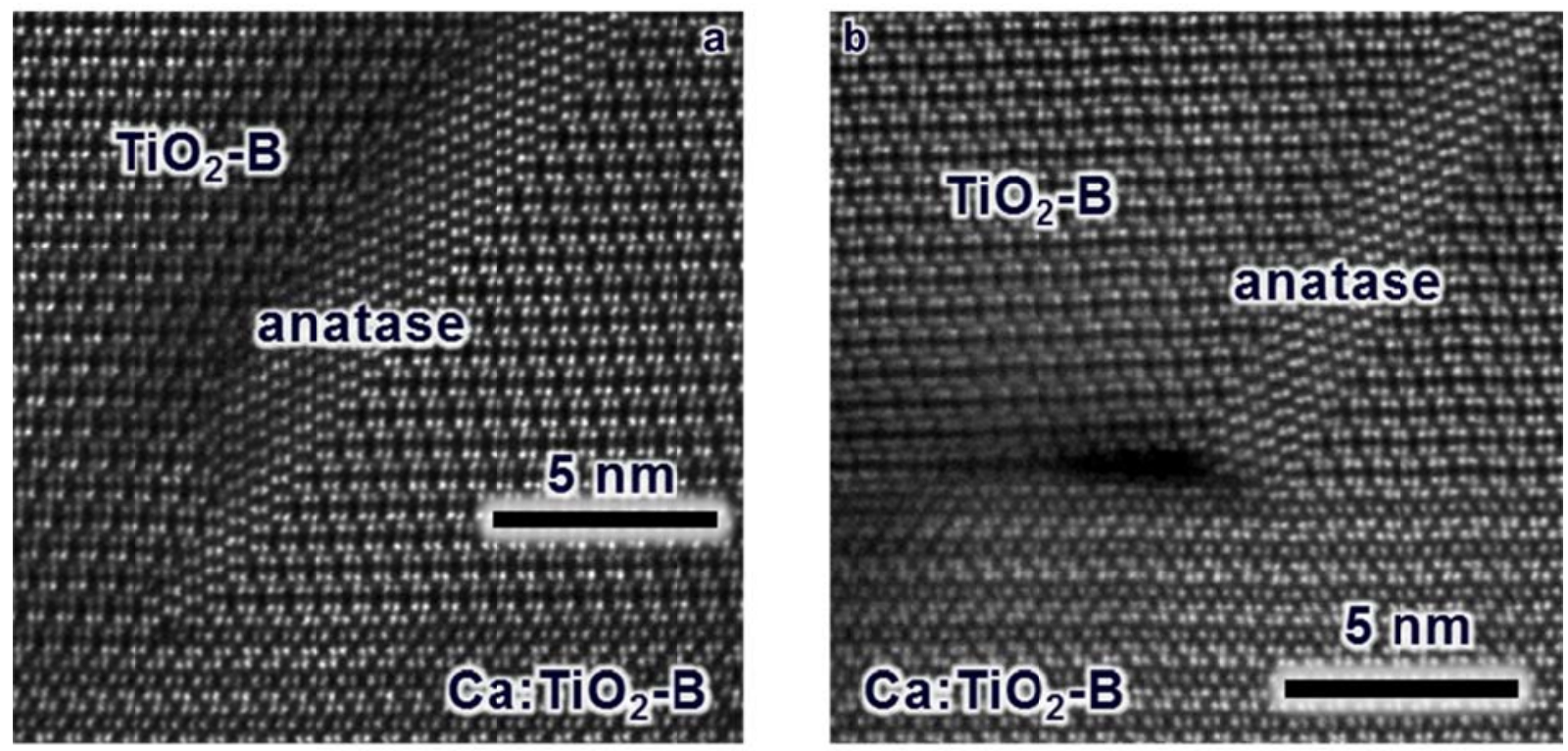

Figure 4.17 Before and after cycling structural comparison of a (001) $\mathrm{TiO}_{2}-\mathrm{B} / \mathrm{Ca}: \mathrm{TiO}_{2}-\mathrm{B}$ dual layer film grown on (001) $\mathrm{SrTiO}_{3}$. A fracture at the interface is clearly seen. Such fractures were observed throughout the post-cycling film. [TEM image credit: a) Michael B. Katz; b) Sung Joo Kim] 


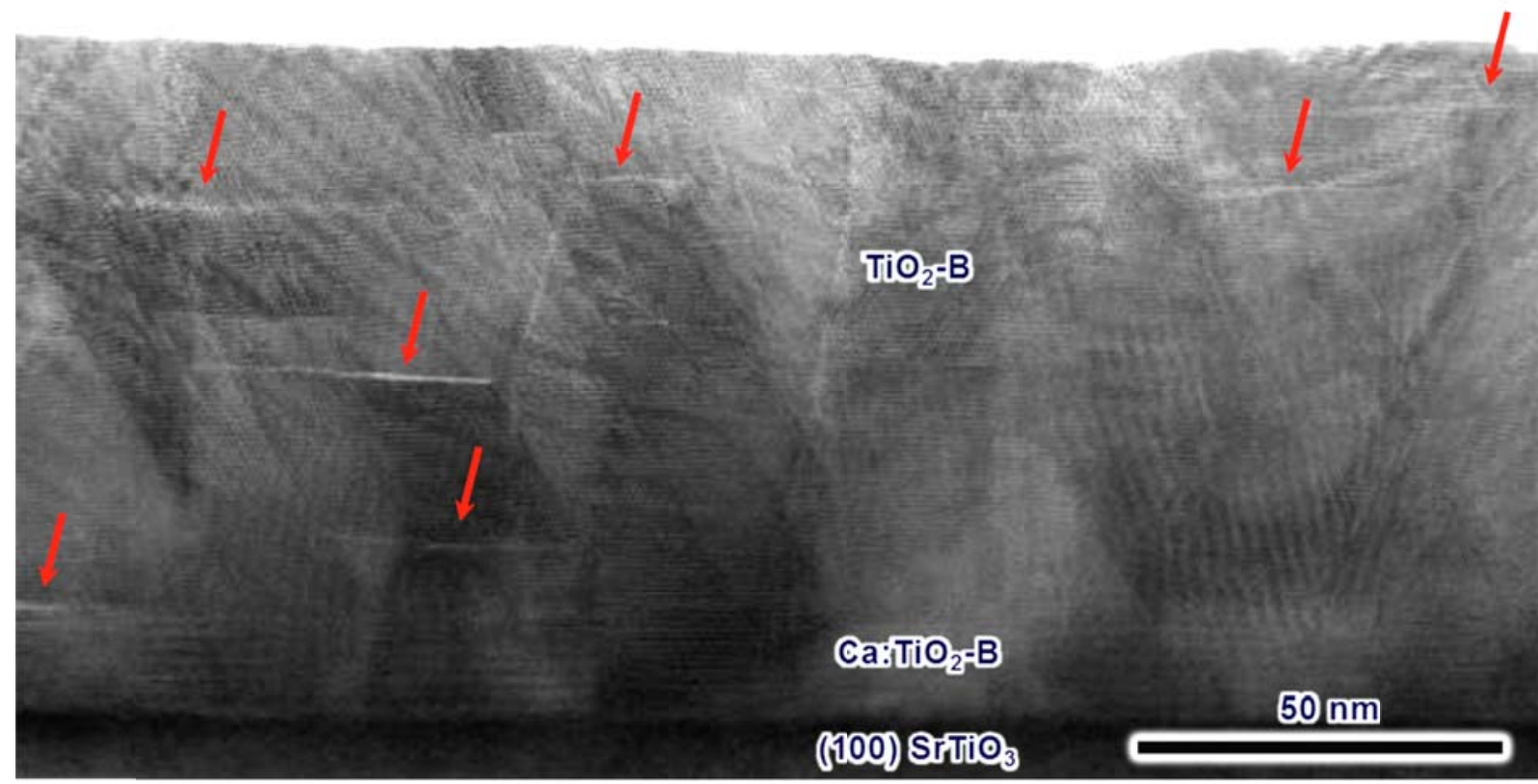

Figure 4.18 Fractures throughout the film caused by charging and discharging: lower magnification STEM image of the same sample as in Figure 4.17(b) showing more fractures in the (001) $\mathrm{TiO}_{2}-\mathrm{B} / \mathrm{Ca}: \mathrm{TiO}_{2}-\mathrm{B}$ dual layer film after cycling. The red arrows point to some of the fractures. [TEM image credit: Sung Joo Kim] 

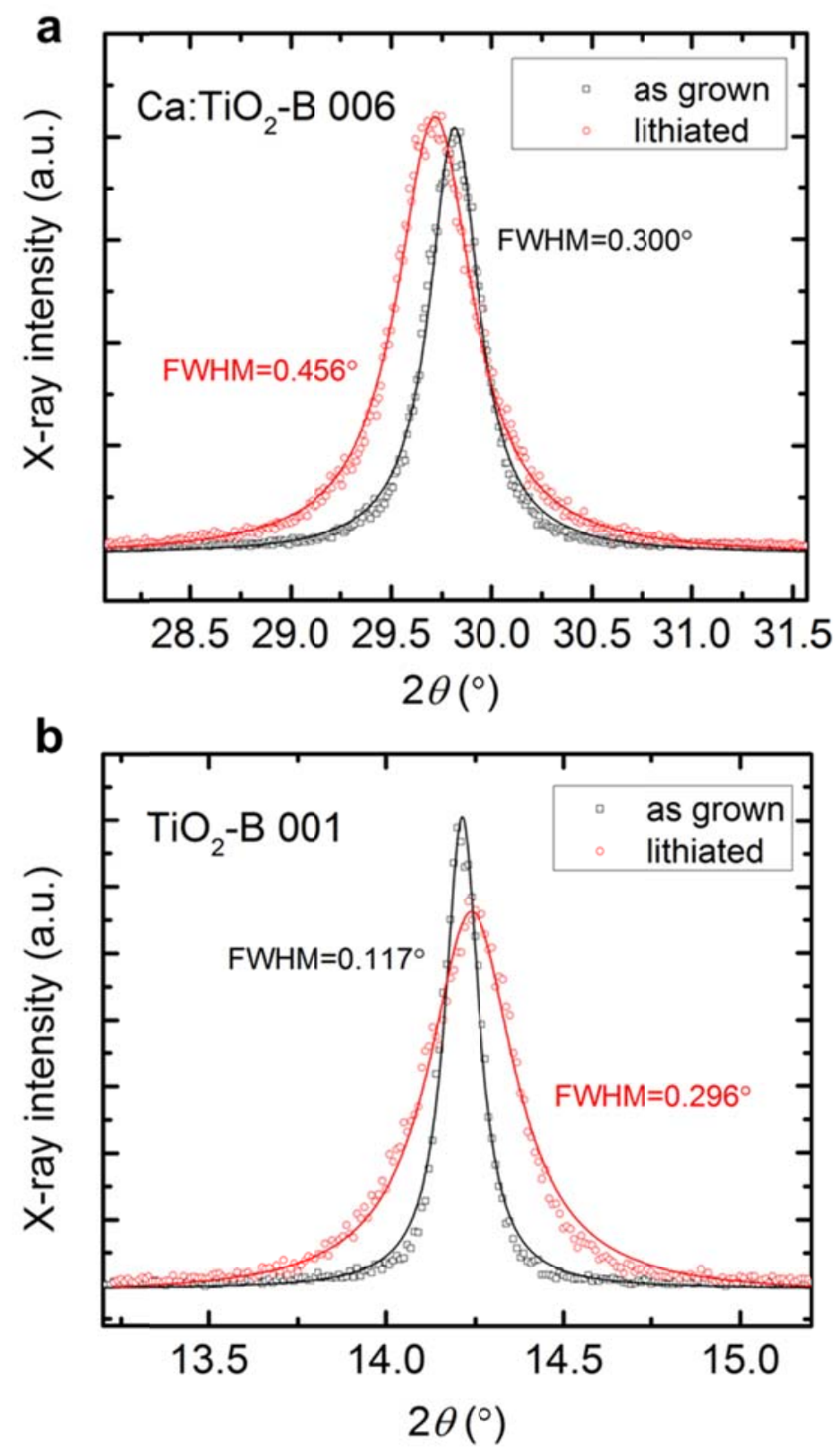

Figure 4.19 Lattice changes due to $\mathrm{Li}^{+}$intercalation. XRD fine scans of (001) bronze films grown on (100) $\mathrm{SrTiO}_{3}$ substrates. Solid curves are fittings of the experimental data. (a) The $\mathrm{Ca}: \mathrm{TiO}_{2}-\mathrm{B} 006$ peak. A peak position shift indicating an increase of $c$ and a peak broadening (FWHM: full width at half maximum) were observed. b) The $\mathrm{TiO}_{2}-\mathrm{B} 001$ peak of a $\mathrm{TiO}_{2}-\mathrm{B} / \mathrm{Ca}: \mathrm{TiO}_{2}-\mathrm{B}$ dual layer film. A peak position shift indicating a slight decrease of $c$, and a peak broadening were observed. 


\section{Chapter 5}

\section{Enhanced High Rate Performance in Ca: $\mathrm{TiO}_{2}-\mathrm{B}$ Thin Films Grown on (110) $\mathrm{SrTiO}_{3}$}

\subsection{Introduction}

The $\mathrm{TiO}_{2}-\mathrm{B}$ material has attracted intensive interest in its application as a battery material specifically because of its high rate performance, a result from its open structure and the pseudocapacitive lithium intercalation process. $^{23} \mathrm{~A}$ few key reports of theoretical and experimental studies on the anisotropic $\mathrm{Li}^{+}$transport in the bronze structure have led the way for the continuous improvement of $\mathrm{TiO}_{2}$-B's rate capabilities. Zukalova et al. (2005) stated that "the key effect seems to be fast $\mathrm{Li}^{+}$transport in the open channels running parallel to the $b$-axis". ${ }^{16}$ Panduwinata et al. (2009) concluded that "the more favorable pathway for diffusion lies close to the center of the more open channel structure, parallel to the $b$ axis of the material in accordance with experimental observations". 133 Arrouvel et al. (2009) published results that revealed "a low energy $(\sim 0.3 \mathrm{eV})$ pathway for Li ion diffusion along the $b$-axis channel in the [010] direction indicating high Li ion mobility." 134 Okumura et al. (2011) proposed that "in lithiated $\mathrm{Li}_{x} \mathrm{TiO}_{2}-\mathrm{B}$, the lithium ions are inserted into the five-fold coordinated sites and/or distorted octahedral sites distributed at the vicinity of $\mathrm{O}$ layers parallel to the ab plane for $\mathrm{x} \leq 0.5$, while the lithium ions are accommodated into the five-fold coordinated site distributed at the vicinity of $\mathrm{TiO}_{2}$ layers parallel to the ab plane for $\mathrm{x}>0.5 \%{ }^{135}$

These findings have pointed out that, $\mathrm{Li}^{+}$access into the $\mathrm{TiO}_{2}-\mathrm{B}$ crystal is expected to be easier in the $a$ - $b$ plane, i.e., through the well-aligned channels along the $a$-axis between layers of 
atoms (Figure 1.1, Figure 4.4(b) and (d)) as well as the possibly even faster channels along the $b$-axis, than in the perpendicular $c$-direction. For the novel $\mathrm{Ca}: \mathrm{TiO}_{2}-\mathrm{B}$ phase, no theoretical predictions on its $\mathrm{Li}^{+}$transport anisotropy are currently available. However, since it shares the similar layered structure to that of the regular $\mathrm{TiO}_{2}-\mathrm{B}$ phase along the $a-b$ planes, the natural deduction is that $\mathrm{Ca}: \mathrm{TiO}_{2}-\mathrm{B}$ may also have fast $\mathrm{Li}^{+}$transport channels within the $a-b$ plane or along its $b$-axis. Future development in the studies of this new material will help clarify the issue.

Previously, we have discussed the electrochemical properties of the (001) $\mathrm{Ca}_{2} \mathrm{TiO}_{2}-\mathrm{B}$ and $\mathrm{TiO}_{2}-\mathrm{B}$ thin films, where the possibly fast $\mathrm{Li}^{+}$transport channels in the $a-b$ plane or along the $b$-axis are all buried in-plane. Since the $\mathrm{Li}^{+}$exchange with the electrolyte occurs on the surface of the film, the rate capability may have been significantly limited. In this chapter, an effort to create fast channel openings on the film surface, and the performance enhancement as a result, will be presented. Owing to the well-defined lattice orientation, the interpretation of the performance data, compared along different crystallographic axes, is more definitive and better supports theoretical studies.

\subsection{Polycrystalline Ca: $\mathrm{TiO}_{2}-\mathrm{B}$ films grown on (110) $\mathrm{SrTiO}_{3}$}

In principle, to have the preferable open channels exposed at the film surface to increase the rates of lithium ion transport can be achieved by utilizing substrates with a different orientation, provided that the epitaxial relationship between the film and the substrate is maintained. Hence, instead of growing on (001) $\mathrm{SrTiO}_{3}$, (110) $\mathrm{SrTiO}_{3}$ substrates were used to deposit $\mathrm{Ca}: \mathrm{TiO}_{2}-\mathrm{B}$ films under the same growth conditions.

Figure 5.1(a) shows a STEM image of a $\mathrm{Ca}: \mathrm{TiO}_{2}-\mathrm{B}$ film deposited on a (110) $\mathrm{SrTiO}_{3}$ substrate from a $\left(80 \% \mathrm{TiO}_{2}+20 \% \mathrm{CaO}\right)$ target. Contrary to the films grown on (001) $\mathrm{SrTiO}_{3}$ which usually have a smooth surface, the surface of this film is quite rough as the $\mathrm{Ca}: \mathrm{TiO}_{2}-\mathrm{B}$ grains are now inclined. Similar to films grown on (001) $\mathrm{SrTiO}_{3}$, anatase grains were again observed near the interface, constituting a small percentage of the volume. Since (001) $\mathrm{Ca}: \mathrm{TiO}_{2}-\mathrm{B}$ films were grown on $\mathrm{SrTiO}_{3}(001)$ surface, it was originally expected that stacking on 
top of the $\mathrm{SrTiO}_{3}(110)$ plane, the atom layers in the $a-b$ planes of these $\mathrm{Ca}: \mathrm{TiO}_{2}-\mathrm{B}$ grains would be parallel to any equivalent $\mathrm{SrTiO}_{3}$ plane in the $\{100\}$ family, all at a $45^{\circ}$ angle with respect to the interface between the film and the substrate. This seemed to be the case at the first glance. As seen in Figure 5.1(c), five $\mathrm{Ca}: \mathrm{TiO}_{2}-\mathrm{B}$ grains with different orientations can be identified, where grains 2 and 3 exhibit a $90^{\circ}$ rotation about the [010] direction from grain 1, and there is also a $90^{\circ}$ rotation about the [001] direction between grains 1 and 4, or between grains 3 and 5, according to the atomic model in Figure 3.7. Such rotations seem to originate directly from the interface with the substrate, while creating crystal defects when two grains meet.

However, the truth is more complicated. A careful examination of the angles between the $a-b$ planes of different grains and the substrate surface, as shown in Figure 5.1(b) and (c), reveals that the angles are not exactly $45^{\circ}$, but a few degrees off. It should be noted that the angles may not be accurate because these STEM images (acquired on a JEOL 2100F) are known to have small $\mathrm{x} / \mathrm{y}$ distortions. The pole figure obtained on this film with the $2 \theta$ angle set at $30.22^{\circ}$ for $\mathrm{Ca}: \mathrm{TiO}_{2}-\mathrm{B} 006$ peak is shown in Figure 5.2(a). Six reflections are relatively strong at $\chi$ angles (Figure 5.2(b)) ranging from $\sim 40-50^{\circ}$, instead of being located exactly at $45^{\circ}$, consistent with the TEM observations. Additional input from theoretical calculations has indicated that it is difficult to build the model of the $\mathrm{Ca}: \mathrm{TiO}_{2}-\mathrm{B}(001)$ plane deposited in $45^{\circ}$ on the $\mathrm{SrTiO}_{3}(110)$ surface with matching bond angle and bond length at the interface. ${ }^{136}$ Therefore, it is believed that the interface between $\mathrm{Ca}: \mathrm{TiO}_{2}-\mathrm{B}$ and $\mathrm{SrTiO}_{3}(110)$ does not conform to a direct epitaxy.

The mystery was solved by a close-up study at the film-substrate interface in TEM. A tiny amount of cubic $\mathrm{CaTiO}_{3}$ phase could be identified at the interface, often manifesting itself in near-triangular shapes likely bounded by its (110) plane and $\{100\}$ family planes, as shown in the STEM image in Figure 5.3(b). To illustrate the relative size of these $\mathrm{CaTiO}_{3}$ grains, one grain is marked by a triangle in Figure 5.1(c). Hence, the growth mechanism of $\mathrm{Ca}^{-} \mathrm{TiO}_{2}-\mathrm{B}$ on (110) $\mathrm{SrTiO}_{3}$ is that pseudo-cubic $\mathrm{CaTiO}_{3}$ grains form first on the substrate surface, and their $\{100\}$ facets provide, again, a (001)-equivalent perovskite plane for the $a-b$ planes of $\mathrm{Ca}: \mathrm{TiO}_{2}-\mathrm{B}$ to build upon. Such an epitaxial relationship is established because the lattice mismatch between 
the $\mathrm{Ca}: \mathrm{TiO}_{2}-\mathrm{B}(001)$ plane and the $\mathrm{CaTiO}_{3}$ (001) plane is small, $3.5 \%$ (Table 3.2). Figure 5.3(a) schematically explains such a mechanism. Adjacent to the $\mathrm{CaTiO}_{3}$ grains, a few layers of regular $\mathrm{TiO}_{2}-\mathrm{B}$ without $\mathrm{Ca}$ were found before the $\mathrm{Ca}: \mathrm{TiO}_{2}-\mathrm{B}$ growth resumed on top (Figure 5.3(b)). This is possibly because the $\mathrm{CaTiO}_{3}$ phase, which has a Ti:Ca ratio of 1:1, took much Ca away from the PLD plume and forced the film to crystallize in the regular $\mathrm{TiO}_{2}-\mathrm{B}$ phase. From another point of view, the $\mathrm{TiO}_{2}-\mathrm{B}$ phase seems to act as a template for the growth of $\mathrm{Ca}: \mathrm{TiO}_{2}-\mathrm{B}$, reversely from what has been presented in previous chapters. Since the growth of the polycrystalline $\mathrm{Ca}: \mathrm{TiO}_{2}-\mathrm{B}$ film occur on facets of numerous small $\mathrm{CaTiO}_{3}$ islands, which are of different sizes and often joining each other at the edges, the large $\mathrm{Ca}: \mathrm{TiO}_{2}-\mathrm{B}$ grains on top exhibit slight variations of their tilting angles from exactly $45^{\circ}$. Due to the nature of its growth, this film contains many grain boundaries (between $\mathrm{Ca}$ : $\mathrm{TiO}_{2}-\mathrm{B}$ grains), interphase boundaries (between $\mathrm{Ca}: \mathrm{TiO}_{2}-\mathrm{B}, \mathrm{TiO}_{2}-\mathrm{B}, \mathrm{CaTiO}_{3}$ and $\mathrm{TiO}_{2}$-anatase), and other crystal defects. These interfacial microstructures and defects are detailed in another study. ${ }^{71}$

\subsection{Enhanced battery performance in $\mathrm{Ca}: \mathrm{TiO}_{2}-\mathrm{B}$ films grown on (110) $\mathrm{SrTiO}_{3}$}

Figure 5.4(b) shows the HAADF image of a $\mathrm{Ca}: \mathrm{TiO}_{2}-\mathrm{B}$ film deposited on a (110) $\mathrm{SrTiO}_{3}$ substrate under the same growth conditions as for the (001) $\mathrm{Ca}: \mathrm{TiO}_{2}-\mathrm{B}$ films on (001) $\mathrm{SrTiO}_{3}$ substrates. Instead of being parallel to the surface, the $a-b$ planes are now inclined, with channels along the $a$ - and $b$-axes reaching the surface. Figure 5.4(a) clearly displays a region near the boundary between two such grains, where one grain (right) has channels parallel to the $a$-axis running to the surface, and another grain (left) is rotated about the $a-b$ plane normal having channels parallel to the $b$-axis running to the surface. Figure 5.4(b) shows the polycrystalline nature of the film. It was found, however, that such a $\mathrm{Ca}: \mathrm{TiO}_{2}-\mathrm{B}$ film does not serve as a good template layer for the subsequent growth of uniform $\mathrm{TiO}_{2}-\mathrm{B}$ on top, due to the lack of a continuous, high quality $\mathrm{Ca}: \mathrm{TiO}_{2}-\mathrm{B}(001)$ plane at the surface.

To study their electrochemical properties, the thin films were assembled in half-cells with metallic $\mathrm{Li}$ as counter electrode. The experimental designs and procedures are detailed in Section 
4.2. For $\mathrm{Ca}: \mathrm{TiO}_{2}-\mathrm{B}$, assuming $5 \mathrm{Li}^{+}$is intercalated per $\mathrm{CaTi}_{5} \mathrm{O}_{11}$ formula unit (making all $\mathrm{Ti}^{+}$), its theoretical capacity is estimated to be $294 \mathrm{~mA} \mathrm{~h} \mathrm{~g}^{-1}$. For simplicity and comparison with $\mathrm{TiO}_{2}-\mathrm{B}$, we define in this chapter $1 \mathrm{C}=335 \mathrm{~mA} \mathrm{~g}^{-1}$. Superior charge/discharge rate capability was observed in the $\mathrm{Ca}: \mathrm{TiO}_{2}-\mathrm{B}$ film grown on (110) $\mathrm{SrTiO}_{3}$ with open channels extending to the surface. Starting at $1 \mathrm{C}$, the battery half-cell was charged and discharged between 1 and $3 \mathrm{~V}$ for 50 cycles at each of several rates up to an extreme of $600 \mathrm{C}$, ending again at $1 \mathrm{C}$ immediately following the last cycle at the highest rate for additional 20 cycles to examine the structural stability. Figure 5.5(a) shows the voltage curves of the 5th cycle at each rate. At $1 \mathrm{C}$, the film discharges to a specific capacity of $293 \mathrm{~mA} \mathrm{~h} \mathrm{~g}^{-1}$, over $99.6 \%$ of the theoretical capacity. The capacity reduced to $248 \mathrm{~mA} \mathrm{~h} \mathrm{~g}^{-1}$ at $10 \mathrm{C}, 61.4 \mathrm{~mA} \mathrm{~h} \mathrm{~g}^{-1}$ at $120 \mathrm{C}$, and $28.8 \mathrm{~mA} \mathrm{~h} \mathrm{~g}^{-1}$ at $600 \mathrm{C}$, likely because only a fraction of the film close to the surface and the current collector was actually lithiated at higher rates. When the rate was lowered back to $1 \mathrm{C}$, the capacity was immediately restored to $284 \mathrm{~mA} \mathrm{~h} \mathrm{~g}^{-1}$, showing outstanding endurance of the material under extreme conditions. The majority of the capacity occurred in the sloped regions of the voltage profiles, while the specific capacity obtained by integrating the cyclic voltammograms was almost the same regardless of the scan rate (Figure 4.15), expressing the pseudocapacitive Faradaic behavior of Li storage in this material.

It should be noted that, an extremely high rate of $12000 \mathrm{C}$ was tested during the experiments, and a discharge capacity of $11.5 \mathrm{~mA} \mathrm{~h} \mathrm{~g}^{-1}$ was achieved. Even though the capacity seems low, it is still believable that at least a portion of the film was lithiated while there was simply not enough time for Li ions to travel far into the bulk of the material, because the capacity was calculated using the entire mass of the film, and it is unlikely that all these electrical charges are stored on the surface alone with exceedingly high density.

To examine its capacity retention characteristics, the battery half-cell containing a $62 \mathrm{~nm}$ $\mathrm{Ca}: \mathrm{TiO}_{2}-\mathrm{B}$ film grown on (110) $\mathrm{SrTiO}_{3}$ was continuously charged and discharged for 200 cycles at $60 \mathrm{C}$ and $80 \mathrm{C}$, as shown in Figure 5.5(b), delivering discharge capacities of $155 \mathrm{~mA} \mathrm{~h} \mathrm{~g}^{-1}$ and $102 \mathrm{~mA} \mathrm{~h} \mathrm{~g}^{-1}$ at the 100th cycle, respectively. The capacity loss and the lower Coulombic 
efficiency in the first 10 cycles reflect the poor electrical conductivity characteristic of pure $\mathrm{Ca}: \mathrm{TiO}_{2}-\mathrm{B}{ }^{33}$ From cycle 10 to 200 , however, the loss was only $0.1 \%$ per cycle, and the Coulombic efficiency close to 1 . For long term cycling performance, the battery was further tested for 2000 cycles at a $60 \mathrm{C}$ rate, and the result is shown in Figure 5.6. Similarly to the above, the Coulombic efficiency was close to 1 other than the first few cycles, and the capacity loss from cycle 10 to 2000 was even lower, at $\sim 0.02 \%$ per cycle.

Furthermore, post-cycling examination of the $\mathrm{Ca}: \mathrm{TiO}_{2}-\mathrm{B}$ film has revealed its extraordinary structural stability after aggressive cycling for more than 60 days, including testing at $12000 \mathrm{C}$ charge/discharge rates, as seen in the HAADF STEM image in Figure 5.4(c). No significant structural degradation or phase transformation, either on the surface or in the film, was observed.

Taking advantage of the clearly defined lattice orientation, we can now experimentally demonstrate the presumed preference for $\mathrm{Li}^{+}$transport along certain crystal directions. The rate capability of $\mathrm{Ca}: \mathrm{TiO}_{2}-\mathrm{B}$ thin films grown on (110) $\mathrm{SrTiO}_{3}$ with channel openings on the surface is compared with both $\mathrm{Ca}: \mathrm{TiO}_{2}-\mathrm{B}$ and $\mathrm{TiO}_{2}-\mathrm{B}$ grown on (100) $\mathrm{SrTiO}_{3}$ with channels along $a$ - and $b$-axes running parallel to the surface. Considering the impaired electron transport in these materials, all three samples were grown to almost the same thickness in order to ensure a fair comparison. Slow cycling tests at a $\mathrm{C} / 10$ rate previously shown in the inset of Figure 5.7 were performed on the two (001) films that have in-plane $a$ - and $b$-channels, where $\mathrm{TiO}_{2}-\mathrm{B}$ and $\mathrm{Ca}: \mathrm{TiO}_{2}-\mathrm{B}$ discharge to $334\left(\mathrm{Li}_{0.997} \mathrm{TiO}_{2}\right)$ and $273 \mathrm{~mA} \mathrm{~h} \mathrm{~g}^{-1}\left(\mathrm{Li}_{4.64} \mathrm{CaTi}_{5} \mathrm{O}_{11}\right)$, respectively. As the rate increases (Figure 5.7), $\mathrm{TiO}_{2}-\mathrm{B}$ delivers higher capacities than $\mathrm{Ca}: \mathrm{TiO}_{2}-\mathrm{B}$ at every rate, indicating that $\mathrm{Li}^{+}$transport along the out-of-plane direction is faster in $\mathrm{TiO}_{2}-\mathrm{B}$ than in $\mathrm{Ca}: \mathrm{TiO}_{2}-\mathrm{B}$, because the difference in theoretical capacity alone is unlikely to account for such discrepancy. On the other hand, the $\mathrm{Ca}: \mathrm{TiO}_{2}-\mathrm{B}$ film with exposed $a$ - and $b$-channels exhibits far superior rate capabilities to both of the above, suggesting a better efficiency of inserting and extracting $\mathrm{Li}^{+}$. The superiority becomes more and more significant with increasing rates. For example, its capacity at $60 \mathrm{C}$ is even higher than the $10 \mathrm{C}$ capacity of $\mathrm{TiO}_{2}-\mathrm{B}$ and the $1 \mathrm{C}$ capacity 
of the same $\mathrm{Ca}: \mathrm{TiO}_{2}-\mathrm{B}$ phase in the different crystal orientation. These results suggest that $\mathrm{Li}^{+}$ transport into the bulk of the material is indeed much faster in the $\mathrm{Ca}_{2} \mathrm{TiO}_{2}-\mathrm{B}$ film on (110) $\mathrm{SrTiO}_{3}$, either because channels within the $a-b$ plane are more favorable for $\mathrm{Li}^{+}$transport than the ones along the $c$-axis, or due to certain effects associated with the polycrystalline structure and grain boundaries.

A preliminary attempt to explain the difference in rate capabilities between the films with different crystal orientations was carried out by EIS (electrochemical impedance spectroscopy) measurements on two $\mathrm{Ca}: \mathrm{TiO}_{2}-\mathrm{B}$ films grown on (110) and (100) $\mathrm{SrTiO}_{3}$, respectively. Experimental data were recorded in a frequency range from $100 \mathrm{kHz}$ to $10 \mathrm{mHz}$ with an $\mathrm{AC}$ perturbation of $\pm 10 \mathrm{mV}$. Nyquist plots are presented in Figure 5.8, where a semicircle at the high frequency region and a straight line at the low frequency region are seen for both. The experimental data has been fitted to the equivalent circuit shown in the inset. The charge transfer resistance $\left(R_{\mathrm{ct}}\right)$ in the film with inclined $a-b$ planes is $30 \%$ lower than that of the other film, consistent with the enhancement in rate capability (Figure 5.7). Cyclic voltammograms were collected for the $\mathrm{Ca}: \mathrm{TiO}_{2}-\mathrm{B}$ film grown on (110) $\mathrm{SrTiO}_{3}$ at scan rates from 0.1 to $1 \mathrm{mV} \mathrm{s}^{-1}$, as shown in Figure 5.9. A pair of redox peaks at $1.42 \mathrm{~V}$ and $1.58 \mathrm{~V}$ was attributed to $\mathrm{Ca}: \mathrm{TiO}_{2}-\mathrm{B}$, while the other two faint peaks at $1.72 \mathrm{~V}$ and $2.34 \mathrm{~V}$ were identified as anatase peaks, resulting from the contribution of the small anatase phase impurities (Figure 5.1). ${ }^{23}$ Compared with (001) $\mathrm{TiO}_{2}-\mathrm{B}$ 's redox peaks at $1.54 \mathrm{~V}$ and $1.69 \mathrm{~V}$ (Figure 4.15), the polycrystalline Ca: $\mathrm{TiO}_{2}-\mathrm{B}$ film requires less energy to lithiate.

In addition to moving along a certain direction, $\mathrm{Li}^{+}$transport in such a polycrystalline $\mathrm{Ca}: \mathrm{TiO}_{2}-\mathrm{B}$ film may also adopt a more complicated pattern combining various diffusion paths. For instance, $\mathrm{Li}^{+}$may hop between different sites in the 3D space along the $a-c$ plane in one grain, followed by a hopping parallel to one of the crystallographic axes, and then enter an adjacent grain and move along yet another direction (Figure 5.4(a)), until the film is lithiated in its entirety. Grain boundaries might have played an important role in the high rate performance in this film, as they could act as diffusion channels for $\mathrm{Li}$ ions due to potentially low $\mathrm{Li}^{+}$migration 
barriers because of structural disorder and incomplete bonding. One might also have noticed that the maximum recorded capacity in the $\mathrm{Ca}: \mathrm{TiO}_{2}-\mathrm{B}$ film grown on (110) $\mathrm{SrTiO}_{3}\left(293 \mathrm{~mA} \mathrm{~h} \mathrm{~g}{ }^{-1}\right.$ at 1C) is higher than that of the film grown on (100) $\mathrm{SrTiO}_{3}\left(273 \mathrm{~mA} \mathrm{~h} \mathrm{~g}^{-1}\right.$ at $\left.\mathrm{C} / 10\right)$, which could possibly be attributed to additional Li sites at the crystal defects. To elucidate the underlying physics of $\mathrm{Li}^{+}$storage and transport in $\mathrm{Ca}: \mathrm{TiO}_{2}-\mathrm{B}$, a new material largely unknown, more in-depth, systematic theoretical and experimental investigations into both single- and poly-crystalline thin films are required. First principles calculations taking into consideration the effects of grain boundaries (with a finite width) and possibly other defects for Li intercalation and migration, instead of simply simulating an infinite, perfect crystal, are currently being conducted. ${ }^{118}$

\subsection{Summary and conclusions}

By depositing on (110) $\mathrm{SrTiO}_{3}$ substrates under the same growth conditions as optimized in Chapter 3, polycrystalline $\mathrm{Ca}: \mathrm{TiO}_{2}-\mathrm{B}$ thin films were grown and characterized. In the absence of a direct epitaxial relationship between $\mathrm{Ca}: \mathrm{TiO}_{2}-\mathrm{B}$ and the $\mathrm{SrTiO}_{3}(110)$ surface, it was found that small pseudo-cubic $\mathrm{CaTiO}_{3}$ islands in near-triangular shapes formed first at the interface, before the $\mathrm{Ca}: \mathrm{TiO}_{2}-\mathrm{B}$ grains started to crystallize with their $a-b$ planes aligned parallel to the (001)-equivalent perovskite facets. Due to the inhomogeneous distribution of these $\mathrm{CaTiO}_{3}$ islands, the $a-b$ planes in the $\mathrm{Ca}: \mathrm{TiO}_{2}$ - $\mathrm{B}$ grains are a few degrees off from being exactly $45^{\circ}$ with respect to the substrate surface.

By aligning the material to a preferred orientation, superior rate capabilities and capacity retention performance were obtained in the $\mathrm{Ca}: \mathrm{TiO}_{2}-\mathrm{B}$ films grown on (110) $\mathrm{SrTiO}_{3}$ with excellent structural stability. At $1 \mathrm{C}$, the film discharges to a specific capacity of $293 \mathrm{~mA} \mathrm{~h} \mathrm{~g}{ }^{-1}$, over $99.6 \%$ of the theoretical capacity. A discharge capacity of $155 \mathrm{~mA} \mathrm{~h} \mathrm{~g}^{-1}$ was achieved at $60 \mathrm{C}$ after 100 cycles. In a long-term retention test at $60 \mathrm{C}$, the capacity loss was $\sim 0.02 \%$ per cycle from cycle 10 to 2000 . 

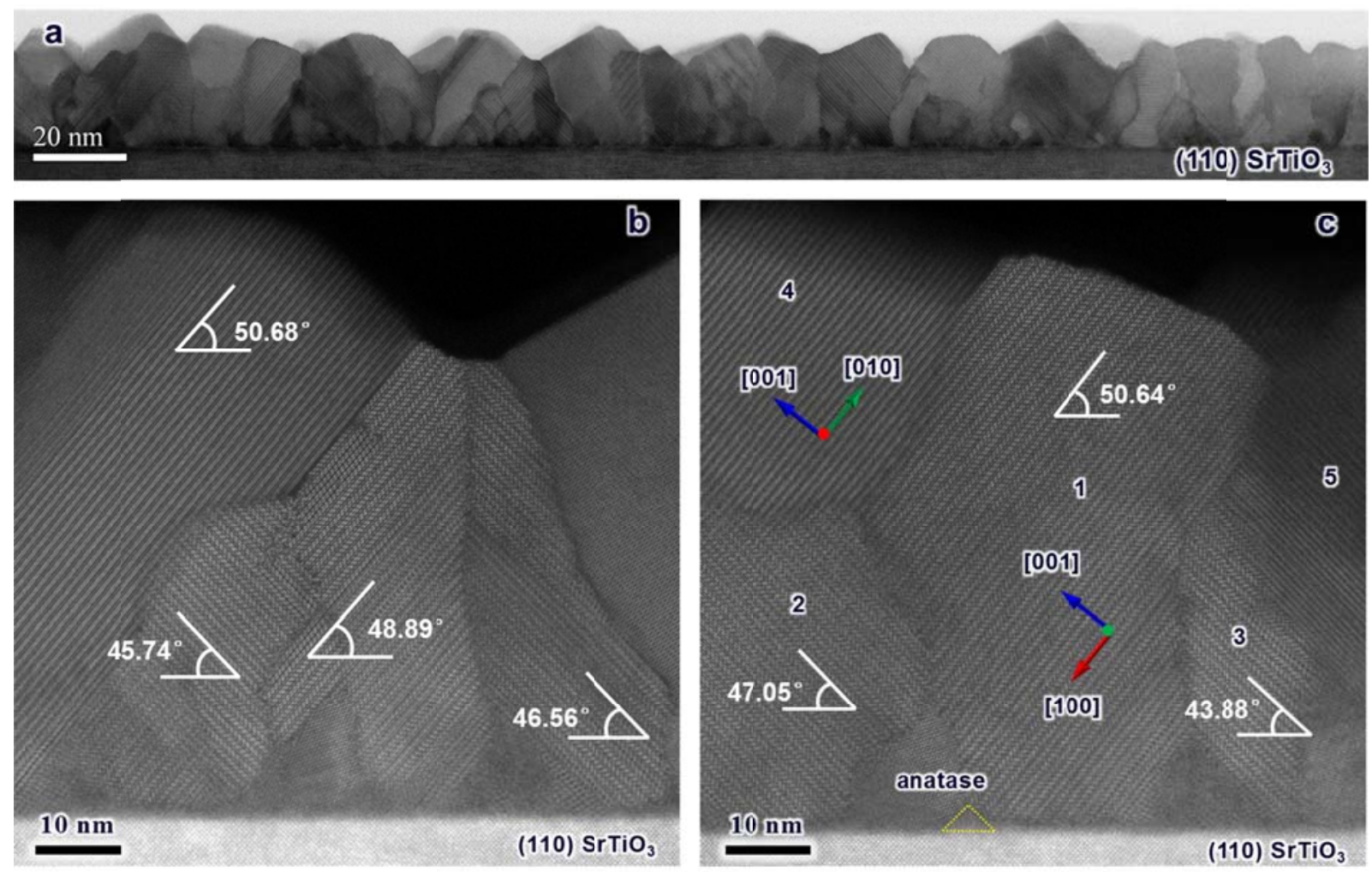

Figure 5.1 (a) Bright field STEM image of a $\mathrm{Ca}: \mathrm{TiO}_{2}-\mathrm{B}$ film grown on a (110) $\mathrm{SrTiO}_{3}$ substrate. (b-c) HAADF STEM images showing different grains in the film. The angles between the $a-b$ planes of different grains and the substrate surface are labeled. The yellow triangle in (c) marks a small $\mathrm{CaTiO}_{3}$ grain at the interface. [TEM image credit: Michael B. Katz] 


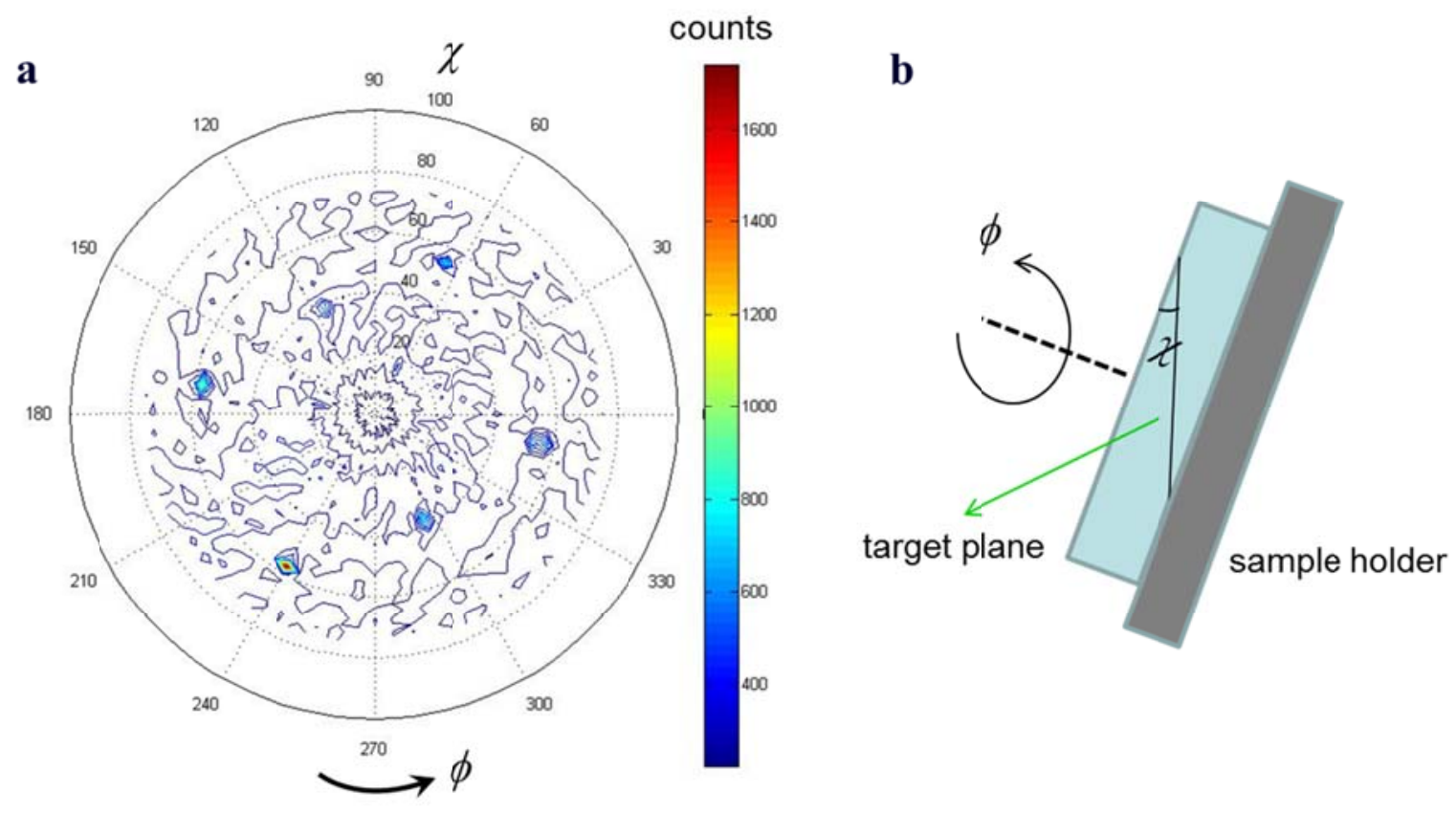

Figure 5.2 (a) Pole figure of the polycrystalline $\mathrm{Ca}: \mathrm{TiO}_{2}-\mathrm{B}$ film grown on a (110) $\mathrm{SrTiO}_{3}$ substrate. The $2 \theta$ angle was set at $30.22^{\circ}$ for $\mathrm{Ca}: \mathrm{TiO}_{2}-\mathrm{B} 006$ peak. The $\phi$ angle was scanned over $0-355^{\circ}$ at a $5^{\circ}$ step; the $\chi$ angle was scanned over $0-75^{\circ}$ at a $2.5^{\circ}$ step. (b) Schematic drawing of the pole figure experiment setup. 

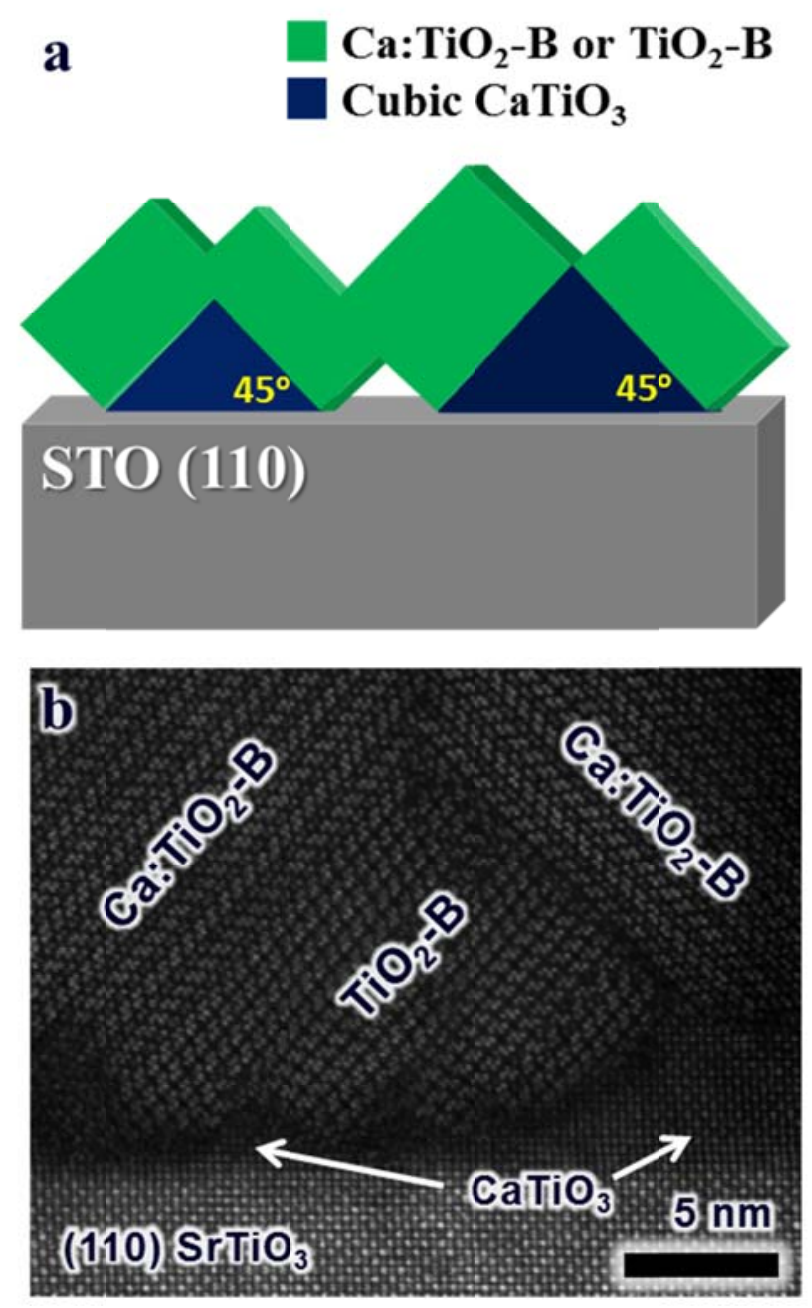

Figure 5.3 (a) Schematic drawing of the bronze film growth on a (110) $\mathrm{SrTiO}_{3}$ substrate. (b) HAADF STEM image showing the cubic $\mathrm{CaTiO}_{3}$ phase formation at the interface. See Ref. 71 for more details. 

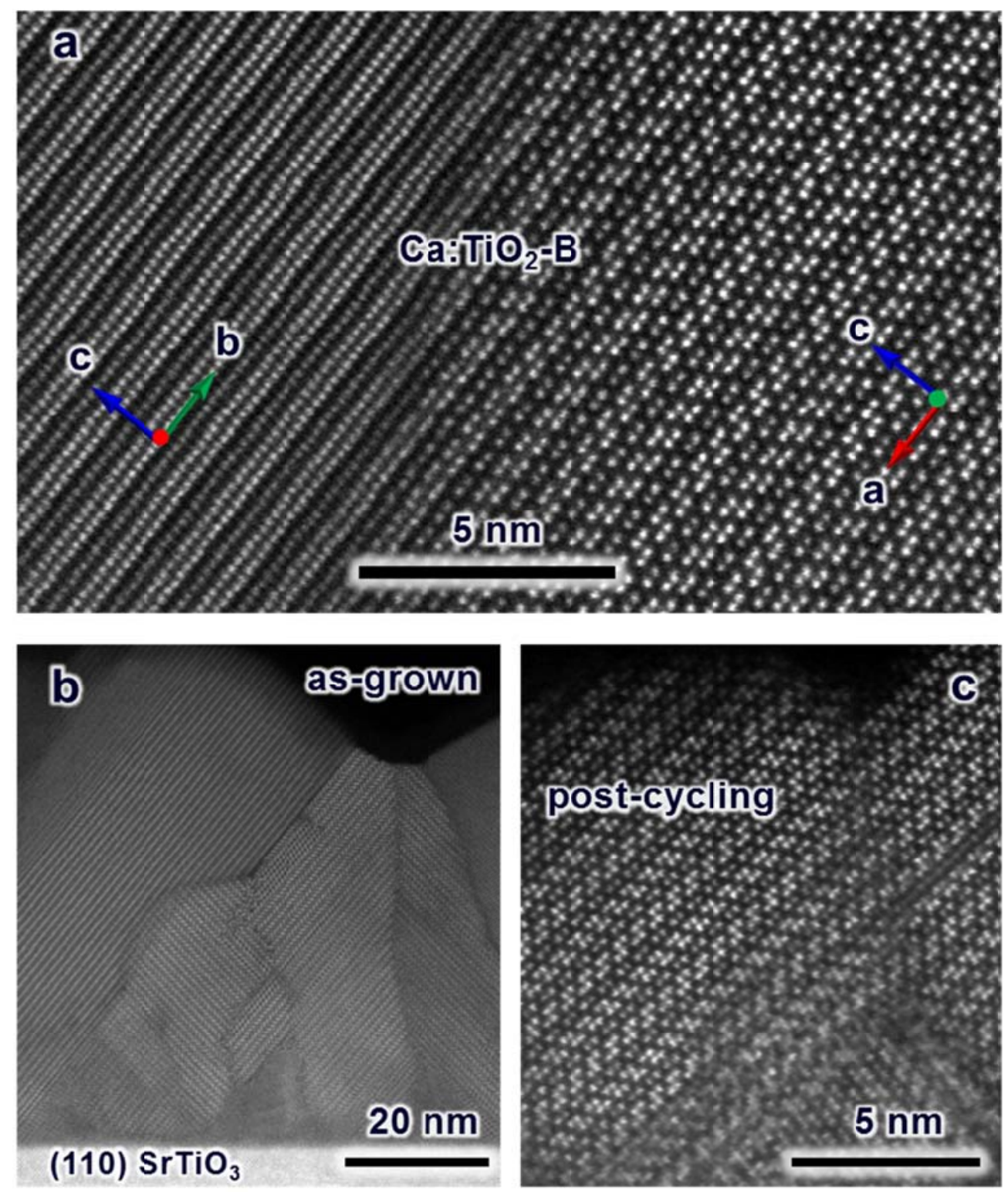

Figure 5.4 Structure an inclined $\mathrm{Ca}: \mathrm{TiO}_{2}-\mathrm{B}$ film grown on a (110) $\mathrm{SrTiO}_{3}$ substrate. a) HAADF STEM image of a region near a grain boundary. b) Lower magnification STEM image showing the polycrystalline nature of the film. Different grains have channels along different directions exposed at the surface. c) HAADF STEM image showing a region near the surface after aggressive cycling for more than 60 days. No significant structural degradation, either on the surface or in the film, was observed. [TEM image credit: Michael B. Katz] 


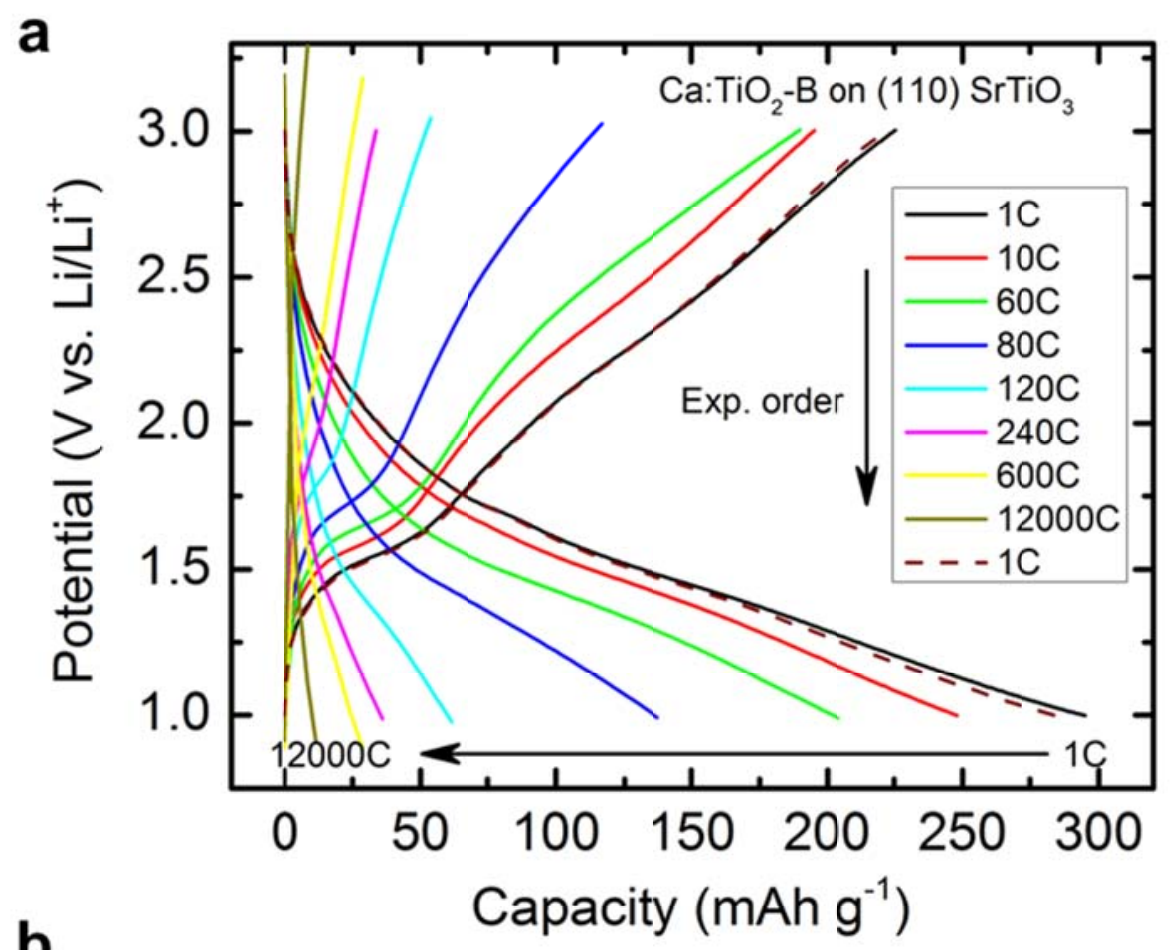

b

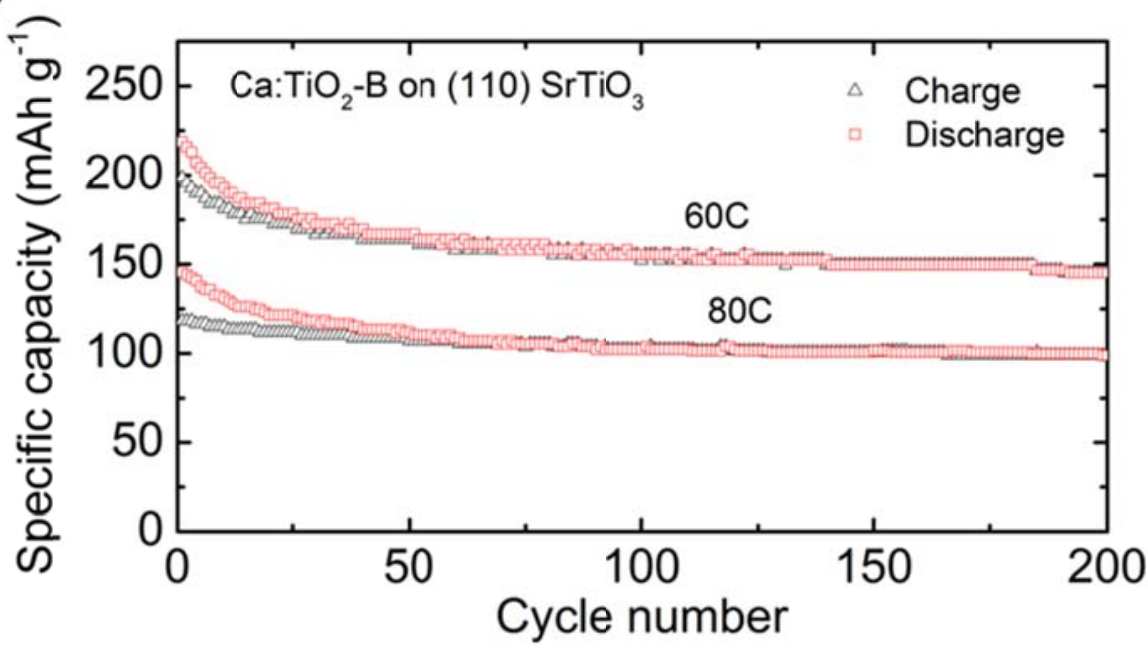

Figure 5.5 Electrochemical performance of an inclined $\mathrm{Ca}: \mathrm{TiO}_{2}-\mathrm{B}$ film grown on a (110) $\mathrm{SrTiO}_{3}$ substrate. (a) Charge-discharge voltage profiles of the 5th cycle at each current rate from 1C to $600 \mathrm{C}\left(1 \mathrm{C}=335 \mathrm{~mA} \mathrm{~g}^{-1}\right)$. Dashed lines depict the profiles at $1 \mathrm{C}$ immediately following the last cycle at the highest rate. (b) Capacity retention at constant $60 \mathrm{C}$ and $80 \mathrm{C}$ rates for 200 cycles in a voltage window of 1-3 V. 


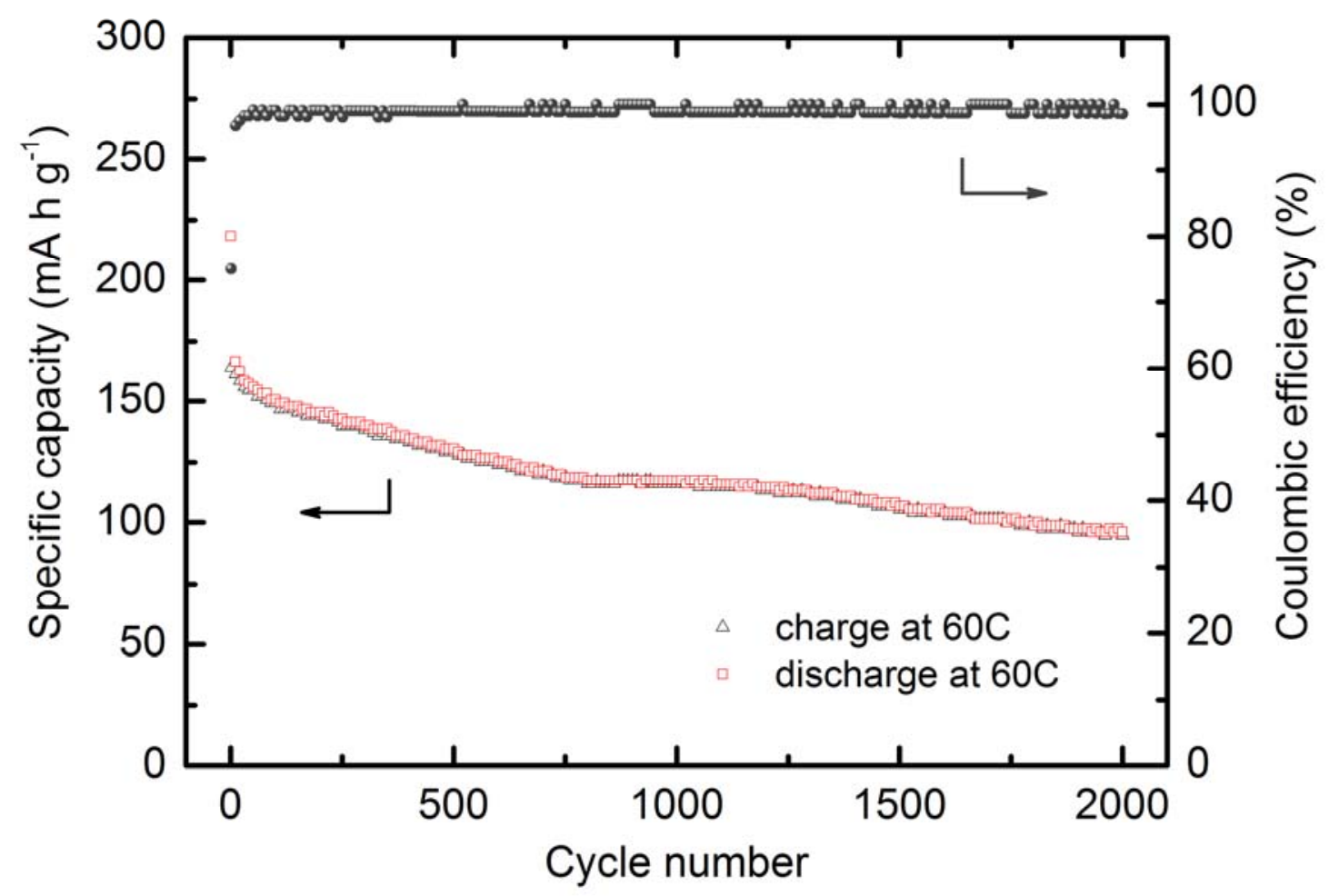

Figure 5.6 Long term cyclability of a $62 \mathrm{~nm}$ polycrystalline $\mathrm{Ca}: \mathrm{TiO}_{2}-\mathrm{B}$ thin film vs. Li metal at a $60 \mathrm{C}$ rate. The voltage window is $1-3 \mathrm{~V}$. 


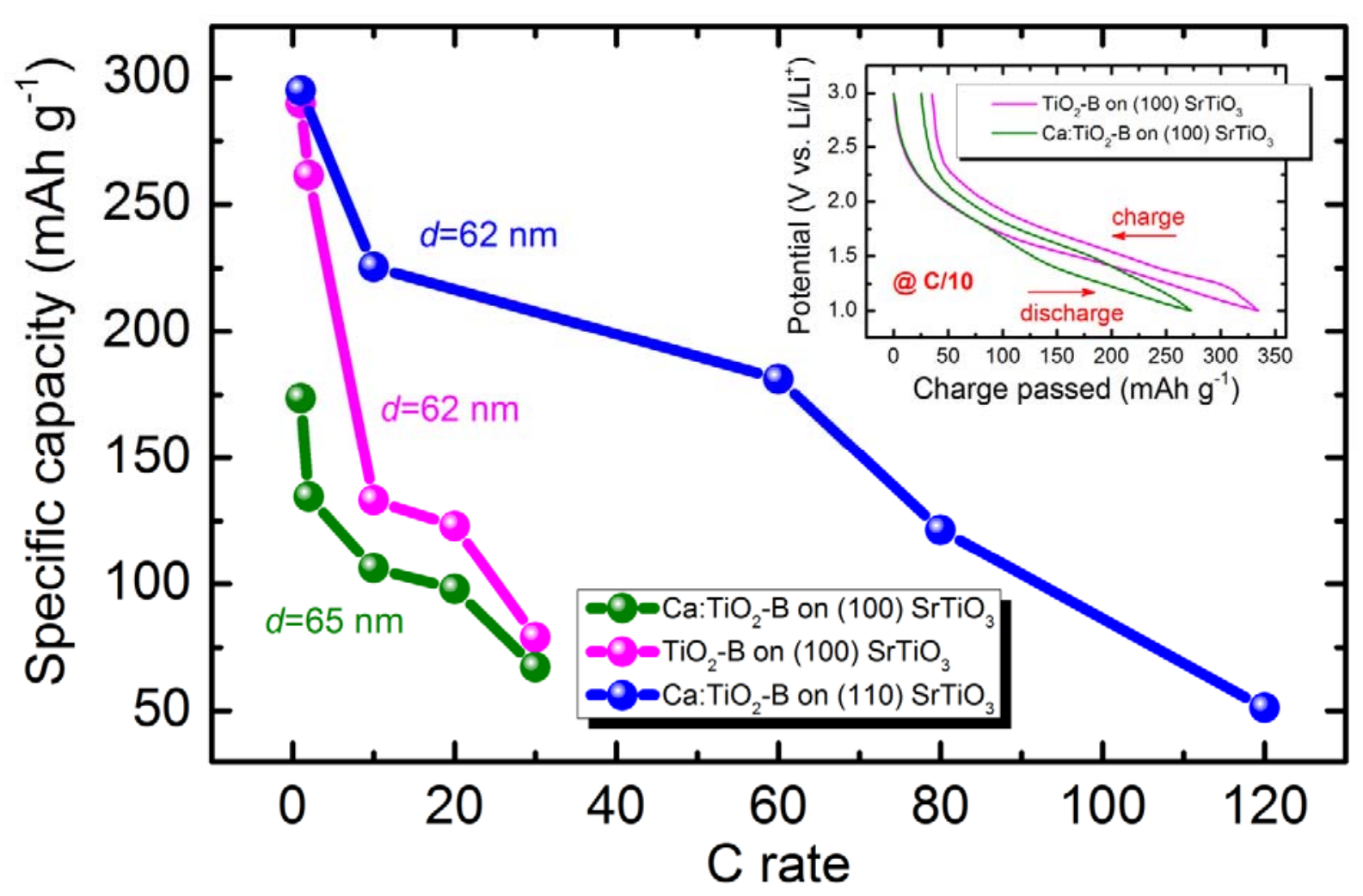

Figure 5.7 Rate capability comparison: discharge capacity at the 20th cycle of both $\mathrm{TiO}_{2}-\mathrm{B}$ and $\mathrm{Ca}: \mathrm{TiO}_{2}-\mathrm{B}$ with different orientation at increasing rates. Respective film thicknesses are labeled. Solid lines are guides for the eyes. Inset: potential profiles at the 5th cycle of (001) $\mathrm{TiO}_{2}-\mathrm{B}$ and $\mathrm{Ca}: \mathrm{TiO}_{2}-\mathrm{B}$ films at $\mathrm{C} / 10$ rate $\left(33.5 \mathrm{~mA} \mathrm{~g}^{-1}\right)$. 


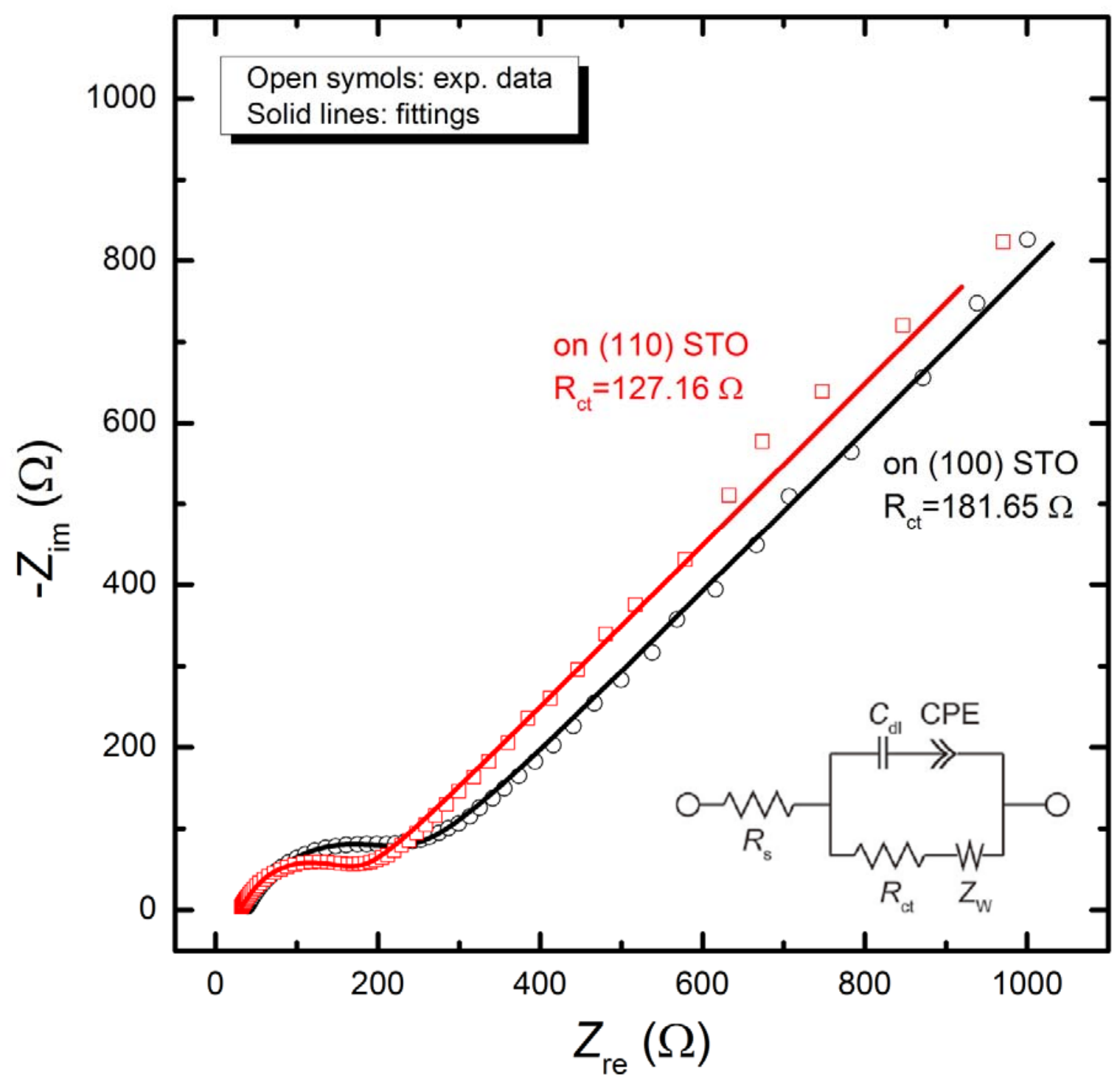

Figure 5.8 EIS spectra comparison between two $\mathrm{Ca}: \mathrm{TiO}_{2}-\mathrm{B}$ films grown on (110) and (100) $\mathrm{SrTiO}_{3}$, respectively. The experimental data (open symbols) has been fitted to the equivalent circuit in the inset, which includes solution resistance $\left(R_{\mathrm{S}}\right)$, an $R C$ circuit representing charge transfer, and a Warburg impedance representing ion diffusion. 


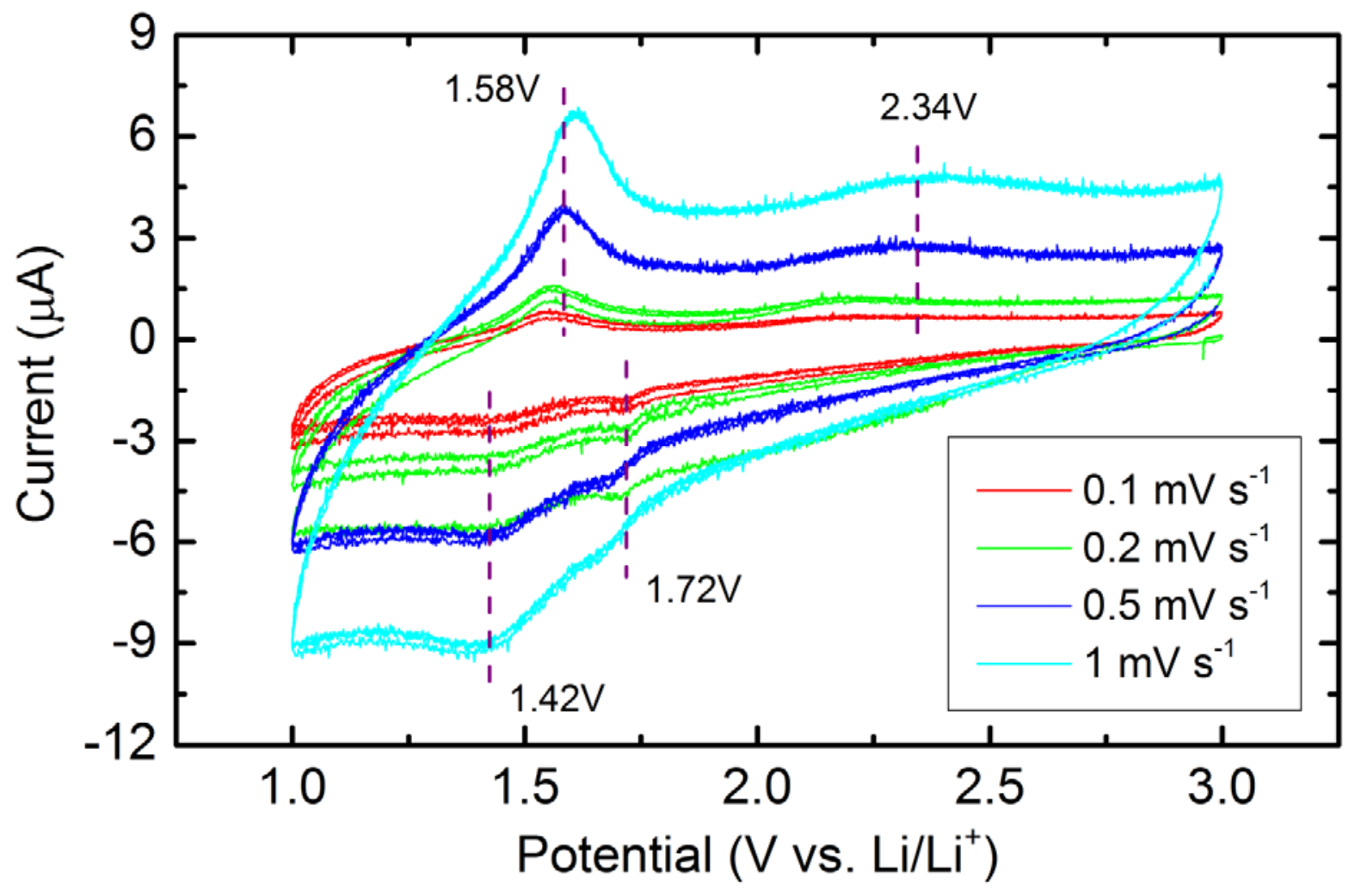

Figure 5.9 Cyclic voltammetry measurements for a polycrystalline $\mathrm{Ca}: \mathrm{TiO}_{2}-\mathrm{B}$ thin film grown on (110) $\mathrm{SrTiO}_{3}$ at different scan rates. Three cycles are shown for each rate, demonstrating good repeatability of the measurement. 


\section{Chapter 6}

\section{High Rate Full-Cell Batteries Containing a Polycrystalline Ca:TiO $-\mathrm{B}$ Anode and $\mathrm{LiFePO}_{4}$ or $\mathrm{LiMn}_{2} \mathrm{O}_{4}$ Cathodes}

\subsection{Introduction}

Having established thus far that the new $\mathrm{Ca}: \mathrm{TiO}_{2}-\mathrm{B}$ phase, when grown in polycrystalline form on (110) $\mathrm{SrTiO}_{3}$ substrates, has superior high rate capabilities, it is of interest to further demonstrate its performance in a real-life situation as an anode material in a full-cell lithium-ion battery. Two cathode materials were chosen for this study: $\mathrm{LiFePO}_{4}$ and $\mathrm{LiMn}_{2} \mathrm{O}_{4} \cdot \mathrm{LiFePO}_{4}$ is a well-known 'fast' material that has exhibited ultrafast charging/discharging capability at a rate up to $400 \mathrm{C},{ }^{15}$ which may pair up with the high rate performance of the $\mathrm{Ca}: \mathrm{TiO}_{2}-\mathrm{B}$ films. $\mathrm{LiMn}_{2} \mathrm{O}_{4}$ was chosen because it has a high voltage versus $\mathrm{Li}$, thus partially compensating the voltage loss on the $\mathrm{Ca}: \mathrm{TiO}_{2}-\mathrm{B}$ anode. As discussed earlier, although the high voltage of the $\mathrm{Ca}: \mathrm{TiO}_{2}-\mathrm{B}$ anode ( $\sim 1.5 \mathrm{~V}$ vs. Li) lowers the overall cell voltage, the intrinsic safety is improved in the meantime, combined with other important advantages including low cost, low toxicity, and good cycle life.

The main purpose of this chapter is a proof of concept, so the cathode materials have not been particularly optimized. Nevertheless, both $\mathrm{LiFePO}_{4}$ and $\mathrm{LiMn}_{2} \mathrm{O}_{4}$ were synthesized in powder form and processed into slurry electrodes, and therefore had much larger mass of active materials than the $\mathrm{Ca}: \mathrm{TiO}_{2}$-B thin films. Even though their specific capacities, which is the passed electrical charge divided by the mass of active material, are not as high as that of $\mathrm{Ca}: \mathrm{TiO}_{2}-\mathrm{B}$, the cathodes still have larger total charge capacities than the anode. Since the capacity of the full-cell is essentially limited by the anode, the battery cyclability as well as the 
rate capabilities reported in this chapter are all calculated using the mass of the $\mathrm{Ca}: \mathrm{TiO}_{2}-\mathrm{B}$ thin film. Due to the difference in mass, when a fixed electrical current flows through the battery, a high $\mathrm{C}$ rate for $\mathrm{Ca}: \mathrm{TiO}_{2}-\mathrm{B}$ anode is actually a lower $\mathrm{C}$ rate for the cathode, which alleviates the issue that the cathode materials were not optimized for high rate performance. A rate of $n \mathrm{C}$ corresponds to a full discharge of the battery in $1 / n$ hours. In this case of a limiting anode, the $\mathrm{C}$ rate for the whole battery is equal to the $\mathrm{C}$ rate for the $\mathrm{Ca}: \mathrm{TiO}_{2}-\mathrm{B}$ thin films.

\subsection{Polycrystalline $\mathrm{Ca}: \mathrm{TiO}_{2}-\mathrm{B}$ thin film anode vs. $\mathrm{LiFePO}_{4}$ slurry cathode}

The homemade $\mathrm{LiFePO}_{4}$ power was prepared from $\mathrm{Li}_{2} \mathrm{CO}_{3}$ and $\mathrm{FePO}_{4} \cdot 2 \mathrm{H}_{2} \mathrm{O}$ (Aldrich $>99 \%$ ) following the reaction below:

$$
0.5 \mathrm{Li}_{2} \mathrm{CO}_{3}+\mathrm{FePO}_{4} \cdot 2 \mathrm{H}_{2} \mathrm{O} \stackrel{\Delta 95 \% \mathrm{Ar}+5 \% \mathrm{H}_{2}}{\longrightarrow} \mathrm{LiFePO}_{4}+0.5 \mathrm{CO}_{2}+2 \mathrm{H}_{2} \mathrm{O}
$$

The mixture was heated at $750{ }^{\circ} \mathrm{C}$ for 3 hours and dried on a hot plate set at $120{ }^{\circ} \mathrm{C}$ in an Ar gas flow for 1 day to remove the water content. The $\mathrm{LiFePO}_{4}$ phase formation in the reaction product was confirmed by XRD and TEM.

The $\mathrm{LiFePO}_{4}$ powder was mixed with carbon black as the current collector and PVDF (polyvinylidene difluoride) binder at a weight ratio of $80 \%: 10 \%: 10 \%$ to form slurry, which was then pasted on $\mathrm{Al}$ disks and dried to a solid state.

The $\mathrm{LiFePO}_{4}$ slurry electrode was first tested in a battery half-cell vs. Li metal with $\mathrm{LiPF}_{6}$ electrolyte. Its cycling behavior at a rate of $47.4 \mathrm{~mA} \mathrm{~g}^{-1}$ in a voltage window of $2.7-4.2 \mathrm{~V}$ is shown in Figure 6.1. The discharge capacity is on average $\sim 65 \mathrm{~mA} \mathrm{~h} \mathrm{~g}^{-1}$, which is fairly low for $\mathrm{LiFePO}_{4}$ with a theoretical capacity of $170 \mathrm{~mA} \mathrm{~h} \mathrm{~g}$. This is likely due to the fact that the $\mathrm{LiFePO}_{4}$ powder composition has not been optimized which may contain impurities and unreacted precursors, while the particle sizes are rather large ( $\mu \mathrm{m}$ level), creating longer diffusion paths for Li ions. ${ }^{137}$ Furthermore, computational studies have derived that in $\mathrm{LiFePO}_{4}$, the smallest Li migration energy lies along the [010] 1D tunnel with the continuous chain of edge-sharing $\mathrm{LiO}_{6}$ octahedra. ${ }^{14,138}$ If the [010] channels are not well exposed in the powder 
particles, the cycling performance will also be limited. Nevertheless, the characteristic intercalation and deintercalation voltages and the small polarization $(120.8 \mathrm{mV})$ of $\mathrm{LiFePO}_{4}$ have been observed in the charge-discharge voltage profiles and cyclic voltammograms (Figure 6.2), ${ }^{137,139}$ illustrating that the active material is indeed storing electrical charges as desired, without obvious contribution from other possible impurities.

A polycrystalline $\mathrm{Ca}: \mathrm{TiO}_{2}-\mathrm{B}$ thin film grown on a (110) $\mathrm{SrTiO}_{3}$ substrate was assembled in a top current collection configuration (Figure 4.7) as the anode, which was then stacked with the $\mathrm{LiFePO}_{4}$ slurry cathode, liquid $\mathrm{LiFP}_{6}$ electrolyte and a $1.55 \mathrm{~mm}$ thick glass fiber separator to form a full-cell battery. Because of the much larger mass of the $\mathrm{LiFePO}_{4}$ phase in the cathode than that of the $\mathrm{Ca}: \mathrm{TiO}_{2}-\mathrm{B}$ phase in the anode, $\mathrm{LiFePO}_{4}$ is not the limiting factor of the battery, and hence the battery performance largely reflects the electrochemical properties of $\mathrm{Ca}: \mathrm{TiO}_{2}-\mathrm{B}$. The high rate cycling results of the $\mathrm{Ca}: \mathrm{TiO}_{2}-\mathrm{B}-\mathrm{LiPF}_{6}-\mathrm{LiFePO}_{4}$ battery are presented in Figure 6.3, where the battery discharges to the specific capacity of $168.6 \mathrm{~mA} \mathrm{~h} \mathrm{~g}^{-1}$ and $155.1 \mathrm{~mA} \mathrm{~h} \mathrm{~g}^{-1}$ in the 100th cycle, at rates of $20 \mathrm{C}$ and $40 \mathrm{C}\left(1 \mathrm{C}=294 \mathrm{~mA} \mathrm{~g}^{-1}\right)$, respectively. Such results are consistent with the outstanding high rate performance of the polycrystalline $\mathrm{Ca}: \mathrm{TiO}_{2}-\mathrm{B}$ thin film as discussed in Chapter 5. In the 40C cycling experiment (Figure 6.3(b)), a segment of increasing capacity is observed approximately from cycle \#350 to cycle \#450, and again approximately from cycle $\# 700$, instead of the usual capacity fade as the cycle number increases. The fluctuation in capacity may have originated from the formation of an SEI layer on either one of the metal oxide electrodes. For instance, the generation of $\mathrm{LiOH}$ and its subsequent reversible reaction with $\mathrm{Li}$ to form $\mathrm{Li}_{2} \mathrm{O}$ and $\mathrm{LiH}$ may contribute extra capacity. ${ }^{140}$

Unlike the half-cells containing $\mathrm{Ca}: \mathrm{TiO}_{2}-\mathrm{B}$ and $\mathrm{Li}$ metal where the Coulombic efficient is close to 1 other than the first few cycles (Figure 5.5(b)), in the $\mathrm{Ca}: \mathrm{TiO}_{2}-\mathrm{B}-\mathrm{LiPF}_{6}-\mathrm{LiFePO}_{4}$ battery the discharge capacity is constantly $\sim 5 \%$ lower than the charge capacity at $20 \mathrm{C}$, and the difference increases at 40C (Figure 6.3). Since the Li ions are extracted from the anode and inserted into the cathode during the discharge process (Figure 4.1), the data suggests that at such high rates, the $\mathrm{Li}$ ions are moving out of $\mathrm{Ca}: \mathrm{TiO}_{2}-\mathrm{B}$ rather fast, but are not being inserted into 
$\mathrm{LiFePO}_{4}$ as efficiently. The $\mathrm{LiFePO}_{4}$ electrode, in its currently less than optimal state, is the rate limiting factor of this battery. The effect is even more significant as the rate further increases. In a long term capacity retention test at $80 \mathrm{C}$ for 2000 cycles, as shown in Figure 6.4, the discharge capacity drops far below the charge capacity after 1200 cycles, exhibiting a difference of $\sim 30 \%$. At higher rates, the capacity also decreases faster in the first few cycles, likely due to the poor electrical conductivity both in $\mathrm{Ca}: \mathrm{TiO}_{2}-\mathrm{B}$ and in $\mathrm{LiFePO}_{4}$.

The 1D curved Li diffusion path imposes strong dimensional restriction of Li motion in $\mathrm{LiFePO}_{4}$. Therefore, the very high rate operation has only been possible through the efficient formation of small particles and/or a conductive carbon network. ${ }^{14,141-143}$ On the present stage, a $\mathrm{LiFePO}_{4}$ cathode with specifically modified crystal structure is needed to further enhance the high rate performance of the $\mathrm{Ca}: \mathrm{TiO}_{2}-\mathrm{B}-\mathrm{LiPF}_{6}-\mathrm{LiFePO}_{4}$ battery.

The electrochemical voltage profiles from the 1 st to the 100 th cycle between $0.5 \mathrm{~V}$ and 2 $\mathrm{V}$ for the $\mathrm{Ca}: \mathrm{TiO}_{2}-\mathrm{B}-\mathrm{LiPF}_{6}-\mathrm{LiFePO}_{4}$ battery at a $10 \mathrm{C}$ rate based on $\mathrm{Ca}: \mathrm{TiO}_{2}-\mathrm{B}$ are shown in Figure 6.5. Since the voltage profile of the $\mathrm{LiFePO}_{4}$ cathode (vs. Li) has a well-developed plateau, while the voltage profile of the $\mathrm{Ca}: \mathrm{TiO}_{2}-\mathrm{B}$ anode (vs. Li) is sloped showing a pseudocapacitive characteristic (Figure 5.5(a)), the full-cell voltage as the difference between the two also poses in a sloped profile. The discharge capacity changes from $257 \mathrm{~mA} \mathrm{~h} \mathrm{~g}^{-1}$ at the first cycle to $189 \mathrm{~mA} \mathrm{~h} \mathrm{~g}^{-1}$ at the 50th cycle, and to $172 \mathrm{~mA} \mathrm{~h} \mathrm{~g}^{-1}$ at the 100th cycle under this rate.

\subsection{Polycrystalline $\mathrm{Ca}: \mathrm{TiO}_{2}-\mathrm{B}$ thin film anode vs. $\mathrm{LiMn}_{2} \mathrm{O}_{4}$ slurry cathode}

The $\mathrm{LiMn}_{2} \mathrm{O}_{4}$ power was prepared and made into slurry electrodes on $\mathrm{Al}$ disks by Prof. Bart Bartlett's research group at the Department of Chemistry. The voltage profiles of the $\mathrm{LiMn}_{2} \mathrm{O}_{4}$ electrode vs. Li metal are shown in Figure 6.6. Except for an irreversible capacity loss

at the 1 st cycle, ${ }^{117}$ the material presents good capacity retention at $\sim 100 \mathrm{~mA} \mathrm{~h} \mathrm{~g}{ }^{-1}$, and the characteristic plateaus around $4.1 \mathrm{~V}^{77}$

Similarly to the above, a polycrystalline $\mathrm{Ca}: \mathrm{TiO}_{2}-\mathrm{B}$ thin film grown on a (110) $\mathrm{SrTiO}_{3}$ substrate assembled in a top current collection configuration as the anode was stacked with the 
$\mathrm{LiMn}_{2} \mathrm{O}_{4}$ slurry cathode, liquid $\mathrm{LiFP}_{6}$ electrolyte and glass fiber separator to form a full-cell $\mathrm{Ca}: \mathrm{TiO}_{2}-\mathrm{B}-\mathrm{LiPF}_{6}-\mathrm{LiMn}_{2} \mathrm{O}_{4}$ battery. The high rate cycling results of are presented in Figure 6.7. Since $\mathrm{LiMn}_{2} \mathrm{O}_{4}$ has a higher voltage vs. $\mathrm{Li}$, the voltage window is moved up to between 1.2 and $3 \mathrm{~V}$. At $20 \mathrm{C}$ and $40 \mathrm{C}$, the battery discharges to the specific capacity of $165.8 \mathrm{~mA} \mathrm{~h} \mathrm{~g}^{-1}$ and 150.6 $\mathrm{mA} \mathrm{h} \mathrm{g}{ }^{-1}$ in the 50th cycle, respectively. Unlike $\mathrm{LiFePO}_{4}, \mathrm{LiMn}_{2} \mathrm{O}_{4}$ is less selective on $\mathrm{Li}^{+}$ intercalation pathways, and therefore the Li ions are inserted into the cathode more efficiently during discharge, which directly leads to an improvement on the Coulombic efficiency compared with the $\mathrm{Ca}: \mathrm{TiO}_{2}-\mathrm{B}-\mathrm{LiPF}_{6}-\mathrm{LiFePO}_{4}$ battery. An obvious difference between discharge and charge capacities is seen when the rate is increased to $80 \mathrm{C}$ (Figure 6.8), where the discharge capacity is generally $\sim 8 \%$ lower than the charge capacity.

The capacity retention of this battery is good. As seen in Figure 6.8, the discharge capacity loss is only $0.03 \%$ per cycle from cycle 1 to 1000 . It should be noted, though, this $80 \mathrm{C}$ long term cycling test was immediately following the cycling at $40 \mathrm{C}$. Hence the 1 st cycle here is not factually the first in an independent test, and the initial irreversible loss was already accounted for in the 40C cycling (Figure 6.7(b)).

The electrochemical voltage profiles from the 1 st to the 100 th cycle between $1.2 \mathrm{~V}$ and 2.7 $\mathrm{V}$ for the $\mathrm{Ca}: \mathrm{TiO}_{2}-\mathrm{B}-\mathrm{LiPF}_{6}-\mathrm{LiMn}_{2} \mathrm{O}_{4}$ battery at a $10 \mathrm{C}$ rate based on $\mathrm{Ca}: \mathrm{TiO}_{2}-\mathrm{B}$ are shown in Figure 6.9, presenting again a sloped line shape due to the pseudocapacitive energy storage in $\mathrm{Ca}: \mathrm{TiO}_{2}-\mathrm{B}$. Even though the plateaus in $\mathrm{LiMn}_{2} \mathrm{O}_{4}$ 's voltage profiles are not as fully extended as those of $\mathrm{LiFePO}_{4}$, the voltage of the cathode does not vary much anyway in cycling because the mass of the active material in the cathode is at least ten times larger than that of the $\mathrm{Ca}: \mathrm{TiO}_{2}-\mathrm{B}$ phase. As a result, these voltage profiles are very similar to the ones in Figure 6.5 with $\mathrm{LiFePO}_{4}$, with a major difference in the higher operation cell voltage, in addition to the better capacity retention. The discharge capacity of the battery is $256 \mathrm{~mA} \mathrm{~h} \mathrm{~g}^{-1}$ at the first cycle, which reduces to $212 \mathrm{~mA} \mathrm{~h} \mathrm{~g}^{-1}$ at the 50 th cycle, and to $189 \mathrm{~mA} \mathrm{~h} \mathrm{~g}^{-1}$ at the 100 th cycle under this rate. 


\subsection{Summary and conclusions}

Polycrystalline $\mathrm{Ca}: \mathrm{TiO}_{2}-\mathrm{B}$ thin films grown on (110) $\mathrm{SrTiO}_{3}$ substrates have been tested as anodes in full-cell batteries against $\mathrm{LiFePO}_{4}$ and $\mathrm{LiMn}_{2} \mathrm{O}_{4}$ cathodes with $\mathrm{LiPF}_{6}$ electrolyte. In both cases, the battery cells exhibited high rate performance up to $80 \mathrm{C}$ (based on $\mathrm{Ca}: \mathrm{TiO}_{2}-\mathrm{B}$ ) and good capacity retention up to 1000 cycles.

The $\mathrm{Ca}: \mathrm{TiO}_{2}-\mathrm{B}-\mathrm{LiPF}_{6}-\mathrm{LiFePO}_{4}$ battery works in the voltage window of $0.5-2 \mathrm{~V}$, and delivers a discharge capacity of $257 \mathrm{~mA} \mathrm{~h} \mathrm{~g}{ }^{-1}$ at $10 \mathrm{C}$. The Ca:TiO $-\mathrm{B}-\mathrm{LiPF}_{6}-\mathrm{LiMn}_{2} \mathrm{O}_{4}$ battery works in the voltage window of 1.2-3 V, and delivers a discharge capacity of $256 \mathrm{~mA} \mathrm{~h} \mathrm{~g}^{-1}$ at 10C. The capacity loss in long term cycling from cycle 20 to 1000 is $0.03 \%$ per cycle with $\mathrm{LiFePO}_{4}$, and $0.02 \%$ per cycle with $\mathrm{LiMn}_{2} \mathrm{O}_{4}$.

Because the $\mathrm{Ca}: \mathrm{TiO}_{2}-\mathrm{B}$ thin film anode is the limiting factor in the full-cell, which has distinctive sloped voltage profiles, the rate capabilities and electrochemical voltage profiles for the two batteries are actually similar at different rates using either $\mathrm{LiFePO}_{4}$ or $\mathrm{LiMn}_{2} \mathrm{O}_{4}$ as cathode. The major differences are that $\mathrm{LiMn}_{2} \mathrm{O}_{4}$ provides a $0.7 \mathrm{~V}$ higher cell voltage (and consequently a higher battery power), and that the Coulombic efficiency is higher with $\mathrm{LiMn}_{2} \mathrm{O}_{4}$ than with the non-optimized $\mathrm{LiFePO}_{4}$, where the latter requires dedicated structural engineering to expose its preferred $\mathrm{Li}^{+}$diffusion channels at the surface of the powder particles in order to achieve superior rate performance. 


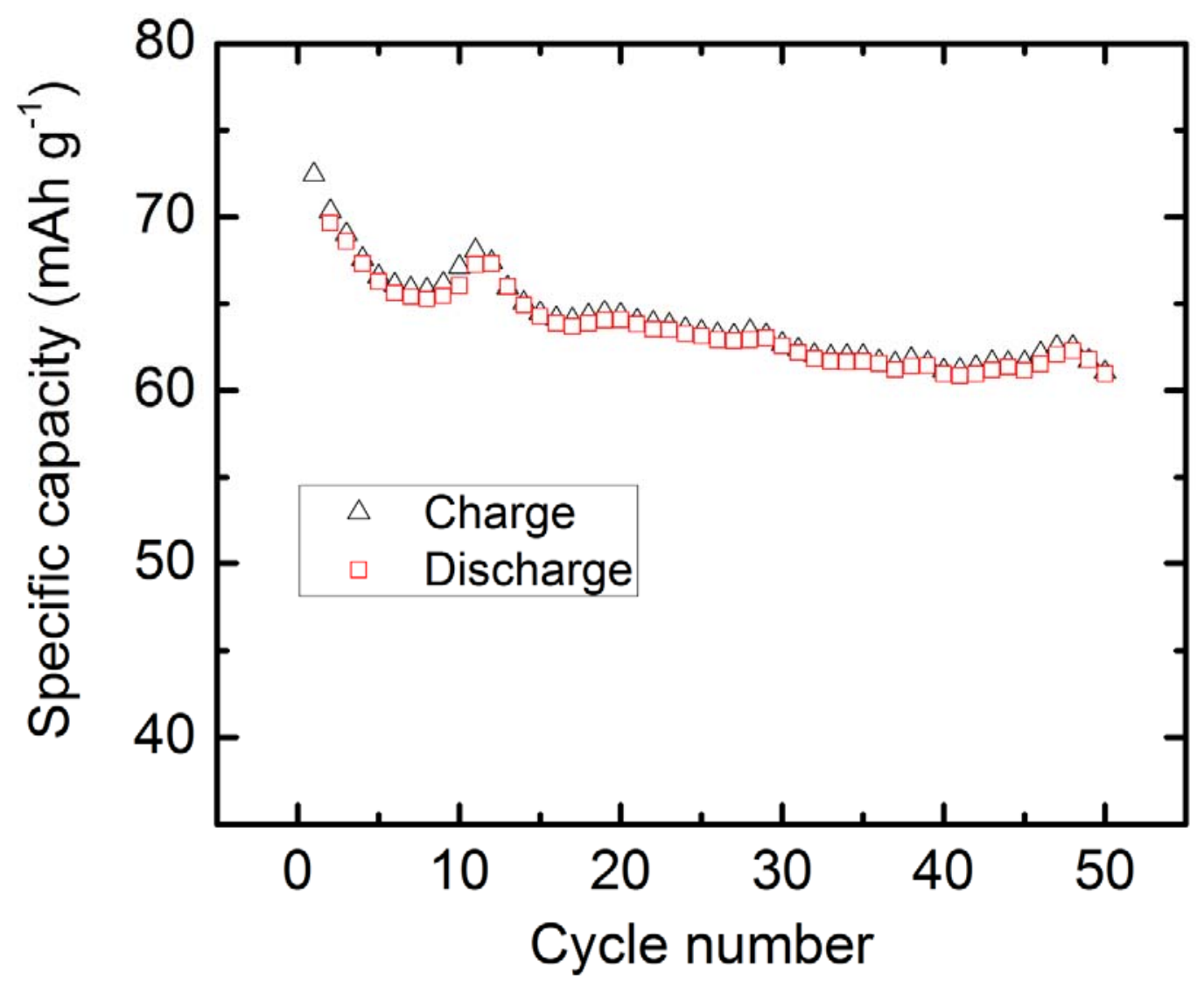

Figure 6.1 Cycling performance of the homemade $\mathrm{LiFePO}_{4}$ slurry electrode vs. $\mathrm{Li}$ counter electrode at a rate of $47.4 \mathrm{~mA} \mathrm{~g}^{-1}$. The voltage window is $2.7-4.2 \mathrm{~V}$. 

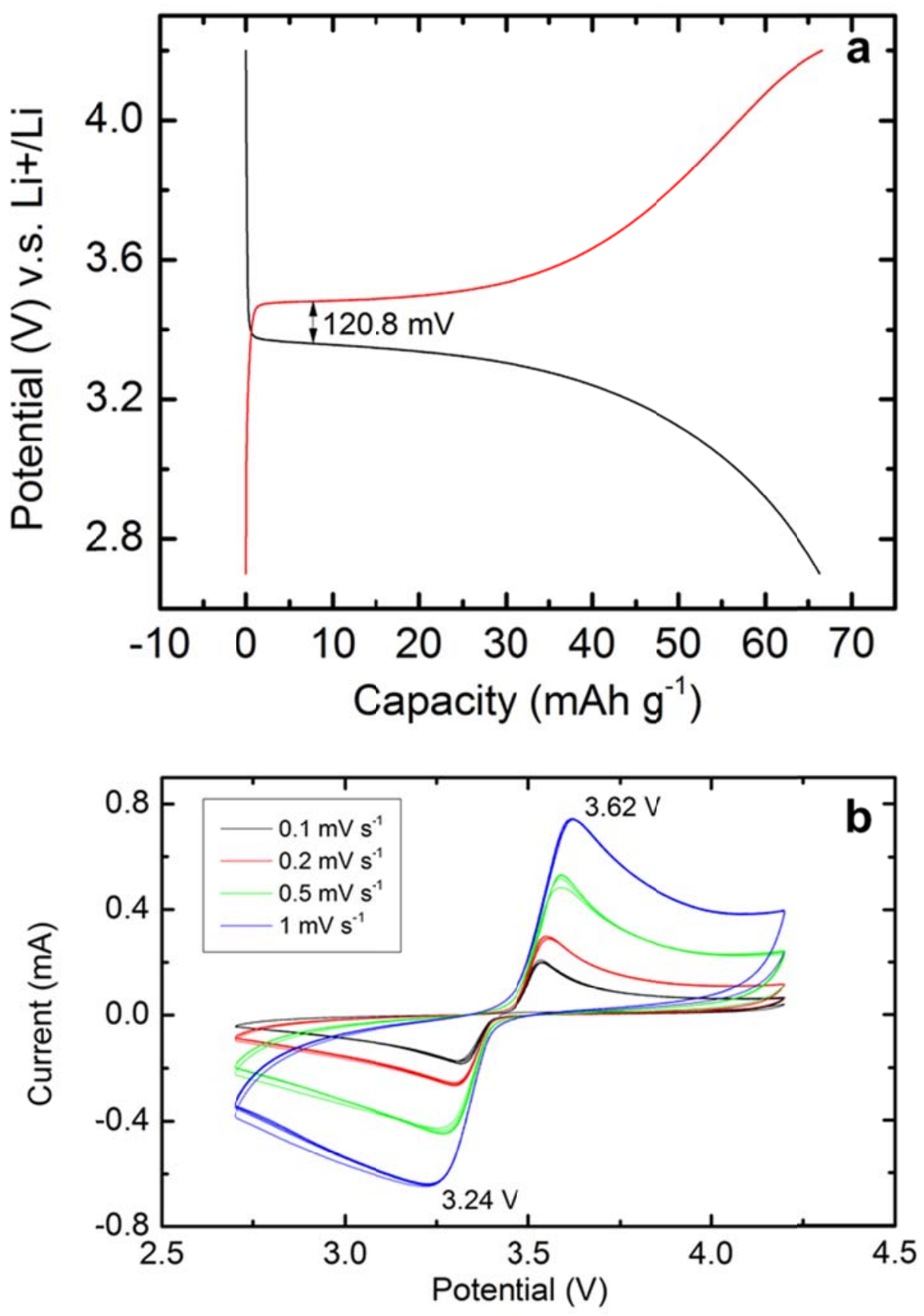

Figure 6.2 (a) Charge-discharge voltage profiles at the 5th cycle of the $\mathrm{LiFePO}_{4}$ slurry electrode vs. $\mathrm{Li}$ counter electrode at a rate of $47.4 \mathrm{~mA} \mathrm{~g}^{-1}$. The voltage window is 2.7-4.2 V. (b) Cyclic voltammograms (CVs) at different scan rates. Five cycles are shown for each rate. 

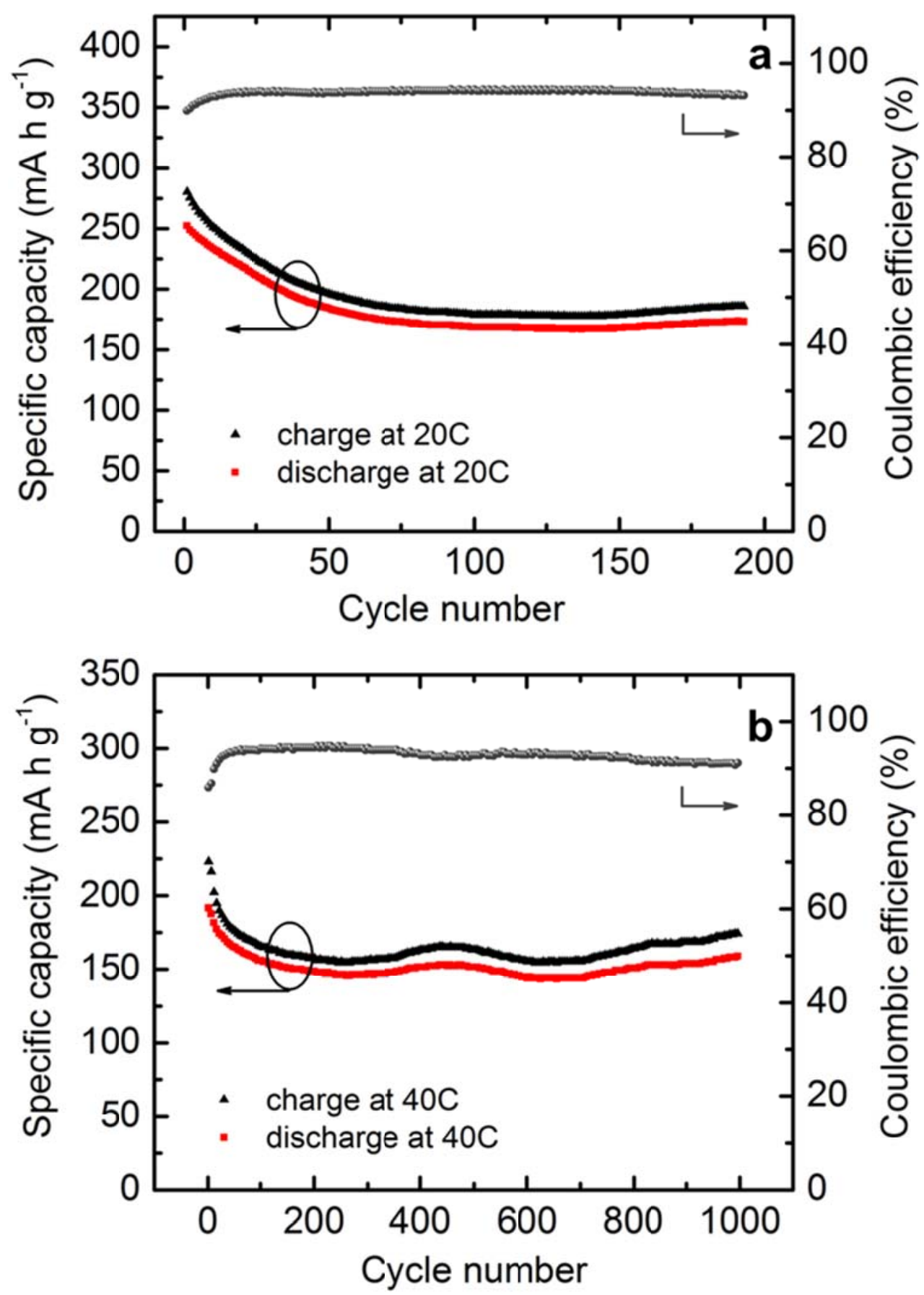

Figure 6.3 Charge and discharge capacities vs. cycle number for the $\mathrm{Ca}: \mathrm{TiO}_{2}-\mathrm{B}-\mathrm{LiPF}_{6}-\mathrm{LiFePO}_{4}$ battery at the rate of (a) $20 \mathrm{C}$, and (b) $40 \mathrm{C}\left(1 \mathrm{C}=294 \mathrm{~mA} \mathrm{~g}^{-1}\right)$. The voltage window is $0.5-2 \mathrm{~V}$. 


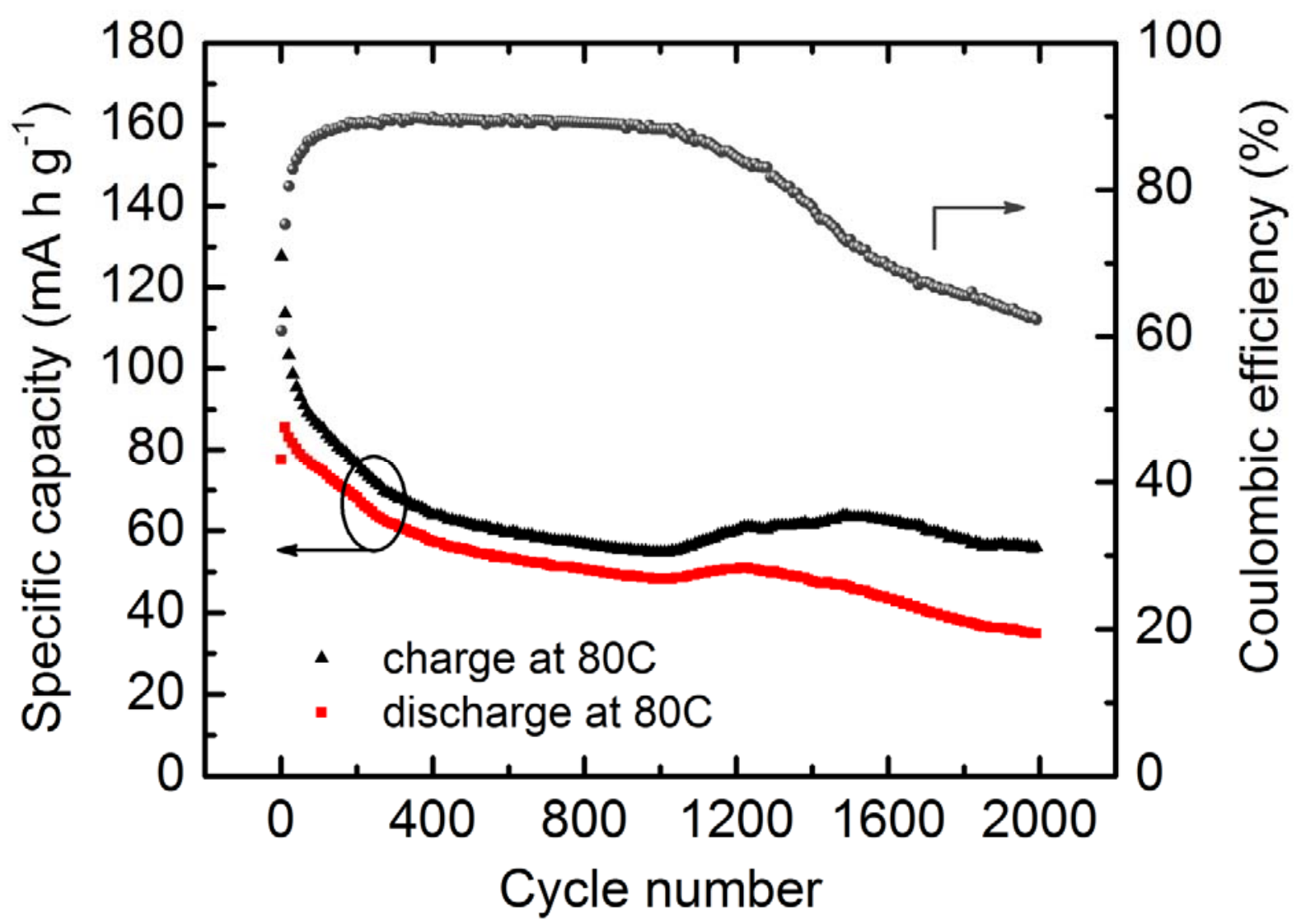

Figure 6.4 Long term capacity retention of the $\mathrm{Ca}: \mathrm{TiO}_{2}-\mathrm{B}-\mathrm{LiPF}_{6}-\mathrm{LiFePO}_{4}$ battery at a $80 \mathrm{C}$ rate $\left(1 \mathrm{C}=294 \mathrm{~mA} \mathrm{~g}^{-1}\right)$. The voltage window is $0.5-2 \mathrm{~V}$. 


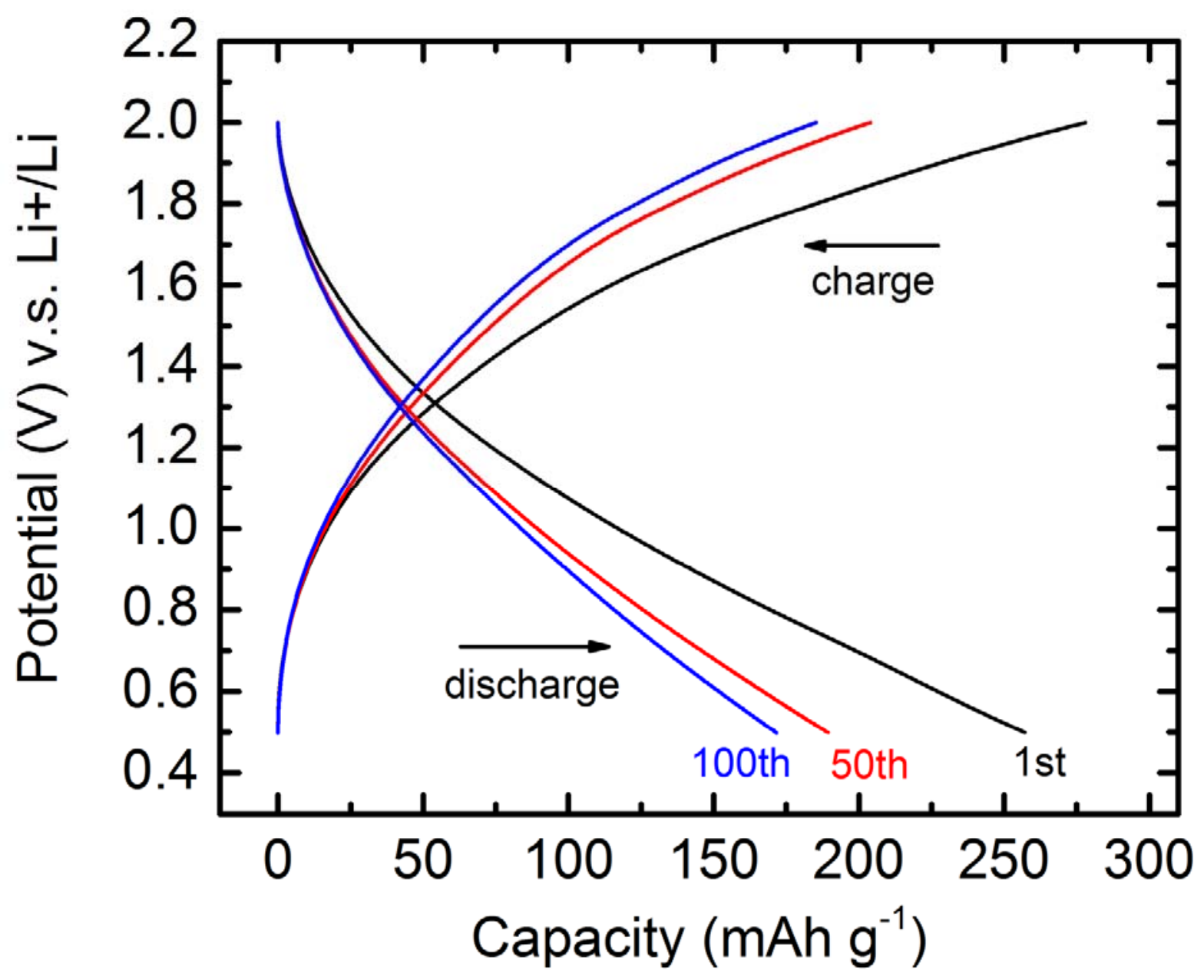

Figure 6.5 Electrochemical voltage profiles at different cycles between $0.5 \mathrm{~V}$ and $2 \mathrm{~V}$ for the $\mathrm{Ca}: \mathrm{TiO}_{2}-\mathrm{B}-\mathrm{LiPF}_{6}-\mathrm{LiFePO}_{4}$ battery at a $10 \mathrm{C}\left(1 \mathrm{C}=294 \mathrm{~mA} \mathrm{~g}^{-1}\right)$ rate based on $\mathrm{Ca}: \mathrm{TiO}_{2}-\mathrm{B}$. 


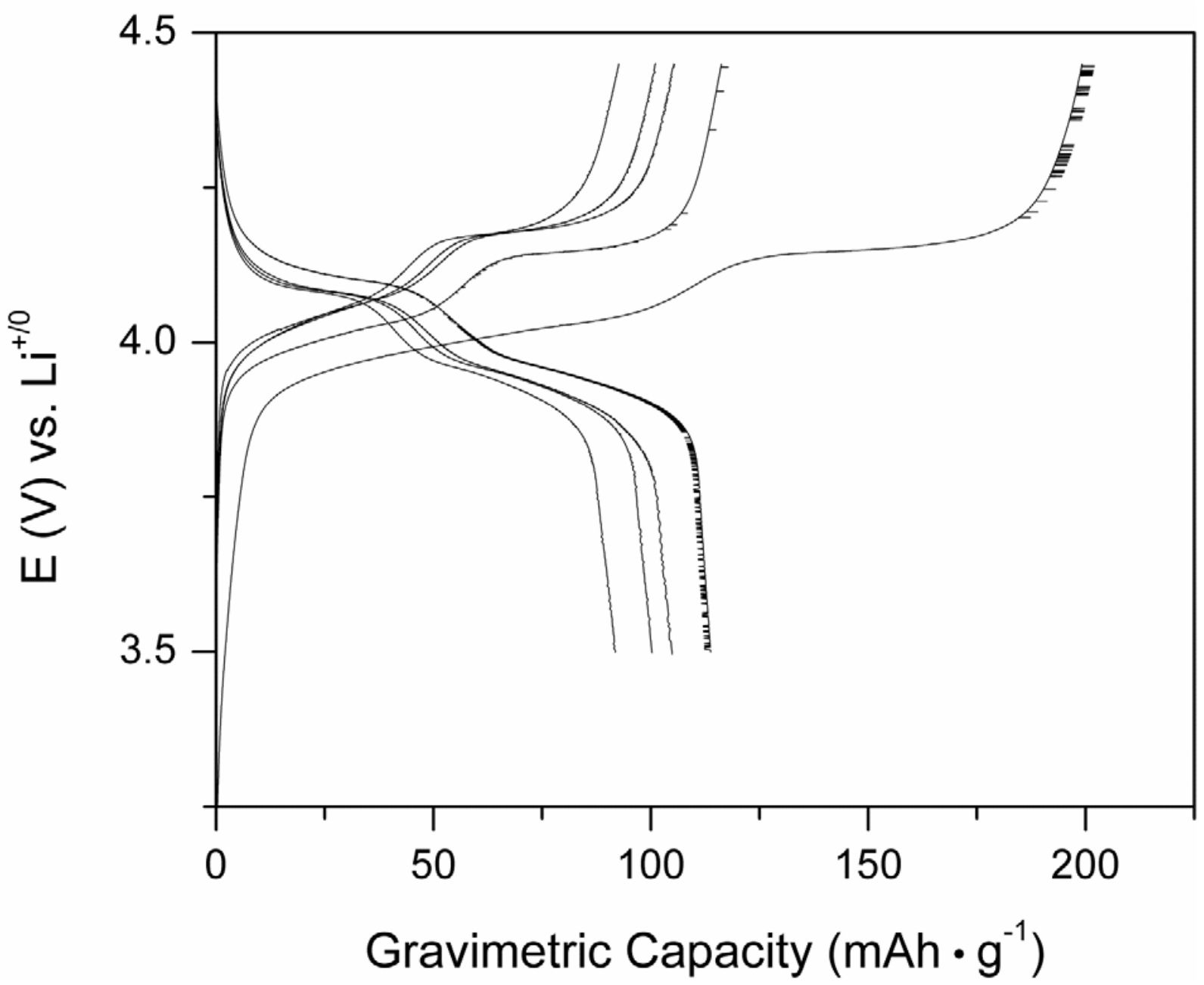

Figure 6.6 Charge-discharge voltage profiles of the $\mathrm{LiMn}_{2} \mathrm{O}_{4}$ slurry electrode vs. $\mathrm{Li}$ counter electrode at $1 \mathrm{C}$ rate from the 1 st cycle to the 150th cycle. [Figure courtesy: Frances Venable and Prof. Bart M. Bartlett] 

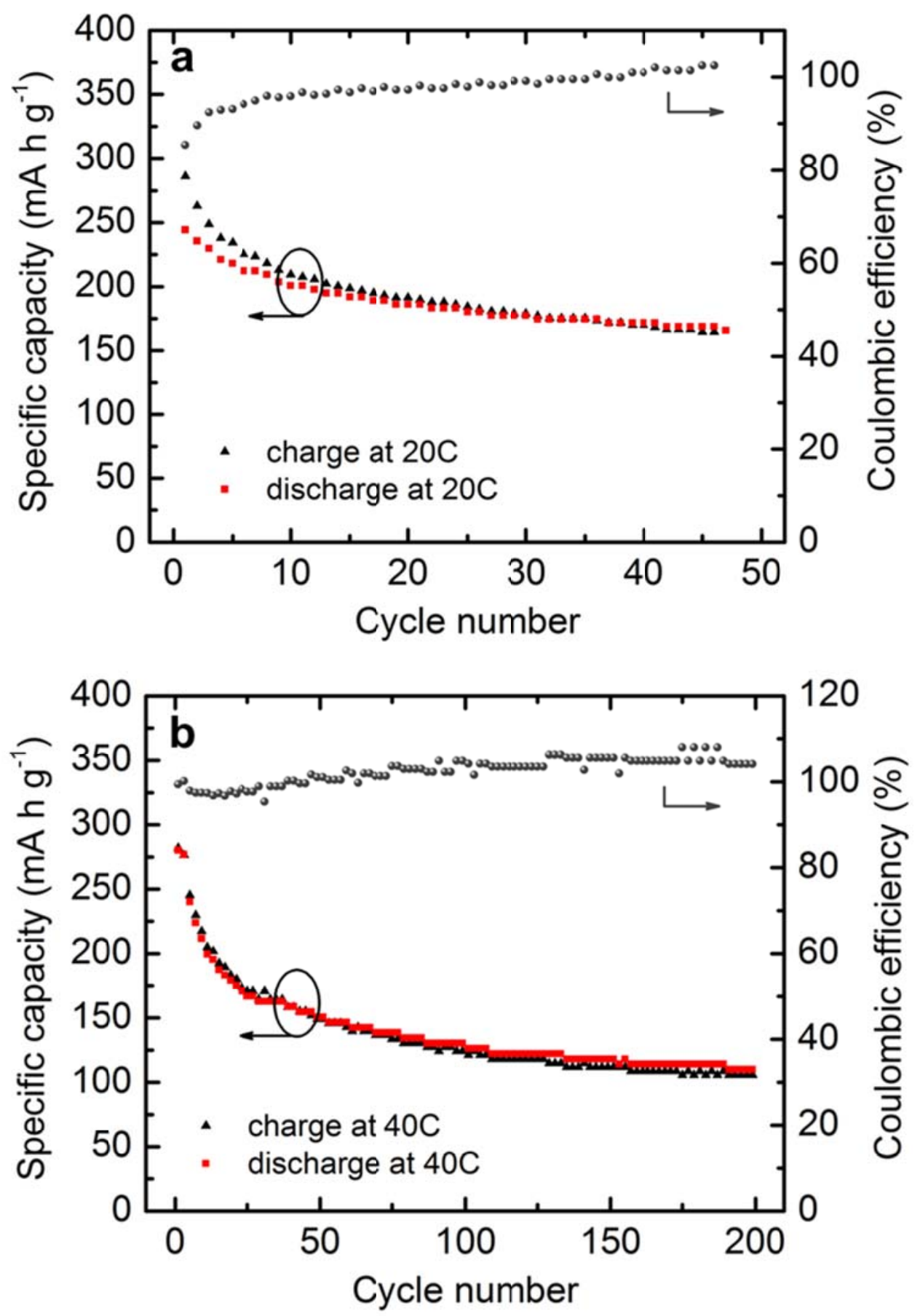

Figure 6.7 Charge and discharge capacities vs. cycle number for the $\mathrm{Ca}: \mathrm{TiO}_{2}-\mathrm{B}-\mathrm{LiPF}_{6}-\mathrm{LiMn}_{2} \mathrm{O}_{4}$ battery at the rate of (a) $20 \mathrm{C}$, and (b) $40 \mathrm{C}\left(1 \mathrm{C}=294 \mathrm{~mA} \mathrm{~g}^{-1}\right)$. The voltage window is $1.2-3 \mathrm{~V}$. 


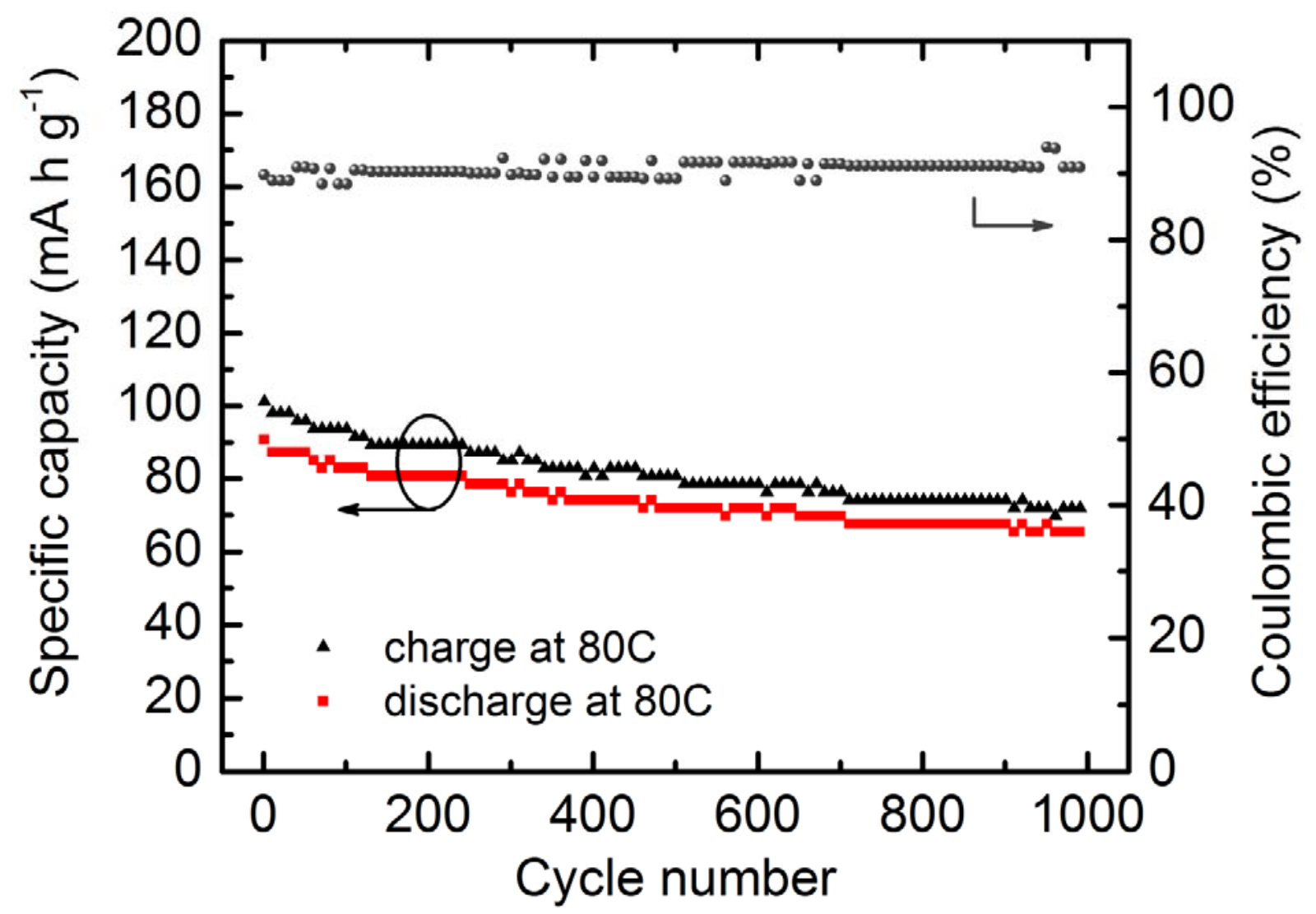

Figure 6.8 Long term capacity retention of the $\mathrm{Ca}: \mathrm{TiO}_{2}-\mathrm{B}-\mathrm{LiPF}_{6}-\mathrm{LiMn}_{2} \mathrm{O}_{4}$ battery at a $80 \mathrm{C}$ rate $\left(1 \mathrm{C}=294 \mathrm{~mA} \mathrm{~g}^{-1}\right)$. The voltage window is $1.2-3 \mathrm{~V}$. 


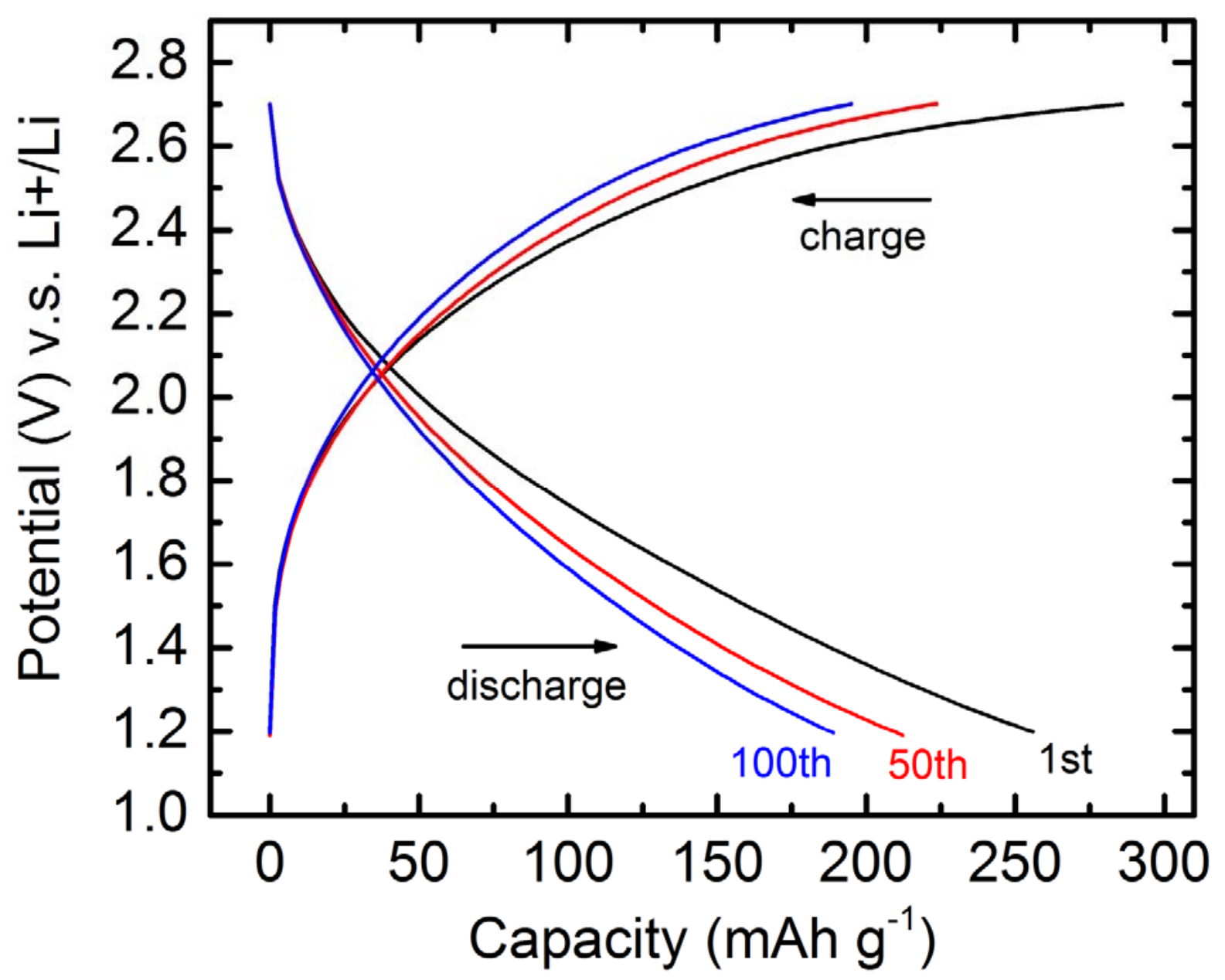

Figure 6.9 Electrochemical voltage profiles at different cycles between $1.2 \mathrm{~V}$ and $2.7 \mathrm{~V}$ for the $\mathrm{Ca}: \mathrm{TiO}_{2}-\mathrm{B}-\mathrm{LiPF}_{6}-\mathrm{LiMn}_{2} \mathrm{O}_{4}$ battery at a $10 \mathrm{C}\left(1 \mathrm{C}=294 \mathrm{~mA} \mathrm{~g}^{-1}\right)$ rate based on Ca:TiO $2-\mathrm{B}$. 


\section{Chapter 7}

\section{Conclusions and Future Work}

\subsection{Thesis conclusions}

This thesis focuses on the first completely waterless synthesis of high quality $\mathrm{TiO}_{2}-\mathrm{B}$ and $\mathrm{Ca}: \mathrm{TiO}_{2}-\mathrm{B}\left(\mathrm{CaTi}_{5} \mathrm{O}_{11}\right)$ thin films, and their application in high rate lithium ion batteries, including a demonstration of high power full-cell battery prototypes. The entire process from epitaxial growth and structural characterization to property testing and performance enhancement has been presented in great detail, with an emphasis on understanding and utilizing the structure-property relationship. The following conclusions are drawn.

\subsubsection{Epitaxial growth of (001) $\mathrm{Ca}: \mathrm{TiO}_{2}-\mathrm{B}$ and (001) $\mathrm{TiO}_{2}-\mathrm{B}$ thin films}

Single-crystalline (001) $\mathrm{Ca}: \mathrm{TiO}_{2}$-B thin films with superior quality and smooth surfaces were achieved on (001) $\mathrm{SrTiO}_{3}$ and doped conductive (001) $\mathrm{Nb}: \mathrm{SrTiO}_{3}$ substrates. The crystal structure matches very well with first principles calculations, where Ca stabilizes the bronze structure and forming a variant phase of $\mathrm{TiO}_{2}-\mathrm{B}$. The $\mathrm{Ca}$ content in the PLD target is critical to the crystallization of such a new phase, with best film quality obtained using a $\mathrm{CaTi}_{4} \mathrm{O}_{9}$ (nominal) target. Deficiency of $\mathrm{Ca}$ in the target leads to $\mathrm{TiO}_{2}$-anatase grains forming near the interface between the film and the substrate.

$\mathrm{Ca}: \mathrm{TiO}_{2}-\mathrm{B}$ may be grown on other substrates with small lattice mismatch and similar crystal symmetry to $\mathrm{SrTiO}_{3}$. Ca: $\mathrm{TiO}_{2}$-B film deposition was observed on (001) $\mathrm{LaAlO}_{3}$ and (001) LSAT, however anatase is a major defect phase. $\mathrm{Ca}: \mathrm{TiO}_{2}-\mathrm{B}$ could also be grown on other 
substrates if a (001) $\mathrm{SrTiO}_{3}$ intervening layer can be fabricated as a buffer. $\mathrm{A} \mathrm{Ca}: \mathrm{TiO}_{2}-\mathrm{B}$ thin film grown on $\mathrm{SrTiO}_{3}$ buffered (100) $\mathrm{Si}$ was achieved and used for Raman studies.

Due to the near-perfect lattice match along the $a-b$ plane, high quality single crystalline (001) $\mathrm{TiO}_{2}-\mathrm{B}$ thin films with smooth surfaces can be synthesized on a (001) $\mathrm{Ca}_{\mathrm{TiO}}-\mathrm{B}$ template layer using a pure $\mathrm{TiO}_{2}$ target. The crystal quality of the $\mathrm{TiO}_{2}-\mathrm{B}$ films is largely independent on the template layer thickness. Depending on the terminating surface in its zigzag structure of the $\mathrm{Ca}: \mathrm{TiO}_{2}-\mathrm{B}$ layer, the $\mathrm{TiO}_{2}-\mathrm{B}$ phase could be stacking upwards along two opposite directions. Therefore, partially missing Ti-Ca alternating layer in the $\mathrm{Ca}: \mathrm{TiO}_{2}-\mathrm{B}$ structure at the interface result in extended defects in the $\mathrm{TiO}_{2}-\mathrm{B}$ film.

\subsubsection{Electrochemical properties of the $\mathrm{Ca}: \mathrm{TiO}_{2}-\mathrm{B}$ and $\mathrm{TiO}_{2}-\mathrm{B}$ thin films}

Both $\mathrm{Ca}: \mathrm{TiO}_{2}-\mathrm{B}$ and $\mathrm{TiO}_{2}-\mathrm{B}$ exhibit $\mathrm{Li}$ electrochemical reactivity, and can be used as anode materials for lithium ion batteries, with a voltage $\sim 1.4-1.7 \mathrm{~V}$.

$\mathrm{Ca}: \mathrm{TiO}_{2}-\mathrm{B}$ has a theoretical capacity of $294 \mathrm{~mA} \mathrm{~h} \mathrm{~g}^{-1}$, with redox peaks located at $1.42 \mathrm{~V}$ and $1.58 \mathrm{~V}$ on $\mathrm{CV}$ curves. A discharge capacity of $273 \mathrm{~mA} \mathrm{~h} \mathrm{~g}^{-1}$ was achieved in a (001) $\mathrm{Ca}: \mathrm{TiO}_{2}-\mathrm{B}$ film. The capacity loss is $0.2 \%$ per cycle from cycle 20 to 200 at a $10 \mathrm{C}$ rate.

$\mathrm{TiO}_{2}-\mathrm{B}$ is known to have a theoretical capacity of $335 \mathrm{~mA} \mathrm{~h} \mathrm{~g}^{-1}$, and a discharge capacity of $334 \mathrm{~mA} \mathrm{~h} \mathrm{~g}^{-1}$ was achieved in a (001) $\mathrm{TiO}_{2}-\mathrm{B}$ film at a rate of $\mathrm{C} / 10$. The electrochemical redox reactions occur at $1.54 \mathrm{~V}$ and $1.69 \mathrm{~V}$.

Both $\mathrm{Ca}: \mathrm{TiO}_{2}-\mathrm{B}$ and $\mathrm{TiO}_{2}-\mathrm{B}$ show a pseudocapacitive intercalation behavior with sloped voltage profiles during $\mathrm{Li}$ intercalation and deintercalation. No well-developed plateau is observed in the voltage profiles. The major loss in capacity during battery cycling, especially in the first few cycles, is from the poor electrical conductivity of these materials.

Structural stability is excellent for both $\mathrm{Ca}: \mathrm{TiO}_{2}-\mathrm{B}$ and $\mathrm{TiO}_{2}-\mathrm{B}$, without any signs of phase transformation in the films, or SEI layer formation or Li plating on the surface after long time aggressive cycling. Small fractures are observed in post-cycling $\mathrm{TiO}_{2}-\mathrm{B}$ films caused by thin layers of as-grown anatase defects. Small crystal deformation upon Li intercalation was 
identified in both materials: a slight increase in the $c$-axis lattice constant of $\mathrm{Ca}: \mathrm{TiO}_{2}-\mathrm{B}$ and a slight decrease in that of $\mathrm{TiO}_{2}$-B. A broadening of the XRD peaks was also observed in both structures as the inserted $\mathrm{Li}^{+}$causes local straining.

\subsubsection{Polycrystalline $\mathrm{Ca}: \mathrm{TiO}_{2}-\mathrm{B}$ thin film with enhanced high rate performance}

Polycrystalline $\mathrm{Ca}: \mathrm{TiO}_{2}$-B thin films can be synthesized on (110) $\mathrm{SrTiO}_{3}$ substrates. Instead of a direct epitaxial growth, small pseudo-cubic $\mathrm{CaTiO}_{3}$ islands in near-triangular shapes form first at the interface, before the $\mathrm{Ca}: \mathrm{TiO}_{2}-\mathrm{B}$ grains crystallize with their $a-b$ planes aligned parallel to the (001)-equivalent perovskite facets. As a result, the $a-b$ planes in the $\mathrm{Ca}: \mathrm{TiO}_{2}-\mathrm{B}$ grains are positioned at an angle of roughly $45^{\circ}$ with respect to the substrate surface.

Such crystal orientation creates possibly fast $\mathrm{Li}^{+}$migration channel openings on the surface of the film, which significantly enhances the rate capabilities and capacity retention of the thin film electrode, delivering specific power of $\sim 20 \mathrm{~kW} \mathrm{~kg}^{-1}$ at $60 \mathrm{C}$ and $\sim 35 \mathrm{~kW} \mathrm{~kg}^{-1}$ at 600C. Post-cycling examinations proved extraordinary structural stability under extreme condition including cycling at $12000 \mathrm{C}$. The superior high rate performance may have benefited not only from the well-aligned lattice orientation, but also from the polycrystalline structure and grain boundaries, where $\mathrm{Li}^{+}$transport follows a mixed pattern.

The outstanding performance was further exploited in prototype full-cell batteries vs. $\mathrm{LiFePO}_{4}$ and $\mathrm{LiMn}_{2} \mathrm{O}_{4}$ cathodes, presenting excellent cyclability up to $80 \mathrm{C}$ (calculated based on $\mathrm{Ca}: \mathrm{TiO}_{2}-\mathrm{B}$ ) and 2000 cycles. Between the two, $\mathrm{LiMn}_{2} \mathrm{O}_{4}$ produced better results due to its $0.7 \mathrm{~V}$ higher voltage and less selective $\mathrm{Li}^{+}$insertion pathways. The $\mathrm{Ca}: \mathrm{TiO}_{2}-\mathrm{B}-\mathrm{LiPF}_{6}-\mathrm{LiFePO}_{4}$ battery is also promising for super high rate batteries with fine-tuned $\mathrm{LiFePO}_{4}$ electrode.

\subsubsection{Impact of this work}

After more than 30 years since the discovery of the $\mathrm{TiO}_{2}-\mathrm{B}$ material, this work reports the first water-free synthesis route to fabricate this attractive material in highly crystalline and phase pure form with well-defined lattice orientation and surface, while in the meantime discovered the 
conditions for its variant phase, $\mathrm{Ca}: \mathrm{TiO}_{2}-\mathrm{B}$, to crystallize.

By clearly illustrating the growth mechanism and various microstructures in the thin films at the atomic scale, our findings can serve as guidelines to create these materials in a controlled form for future research efforts. Further optimized growth conditions, transferring onto other deposition platforms such as molecular beam epitaxy (MBE), faster deposition rates to gain larger yield, morphology engineering such as nanostructuring, may be possible. Building on the investigation of their electrochemical properties provided here, the perspective to further enhance the battery performance is promising.

Although this thesis has emphasized on the application of these materials as strong candidates for high power lithium ion battery anodes, their potential applications extend far beyond electrochemistry. The large unit cells of $\mathrm{Ca}: \mathrm{TiO}_{2}-\mathrm{B}$ may warrant good thermoelectric properties. The clean surface is especially beneficial to studies focused on surface states, such as in photocatalysis and photovoltaic devices.

Compared with other reports on $\mathrm{TiO}_{2}-\mathrm{B}$ synthesized by hydrothermal methods into powder forms, the unparalleled advantage of the crystalline thin films is a determinate relationship between structure and property, thus providing a solid baseline for experimental data interpretation and association with theoretical studies. This in itself is a significant step forward in understanding the fundamental science of these materials.

\subsection{Future work}

Many aspects of the titania-bronze materials, from the structure to underlying physics and chemistry, especially for the novel $\mathrm{Ca}: \mathrm{TiO}_{2}-\mathrm{B}$ phase, are currently uncharted waters. The high quality single crystals offer unique opportunities to answer these questions. A few recommendations for immediate next steps are introduced below. 


\subsubsection{Thicker films}

In the growth of the $\mathrm{TiO}_{2}-\mathrm{B}(001)$ films, it was noticed that the deposition rate slows down significantly after about 2 hours of growth. Within a $100 \mathrm{~nm}$, the thickness increases roughly proportional to growth time. However, further increasing the deposition time up to 6 hours did not result in obvious addition to the film thickness. So far, no one film has a thickness above $250 \mathrm{~nm}$.

Preliminary analysis indicates a critical thickness due to unrelaxed strain. Being a metastable phase, the building blocks of $\mathrm{TiO}_{2}-\mathrm{B}$ might stop to stack when the internal strain reaches a threshold. One possible solution is to insert more layers of $\mathrm{Ca}: \mathrm{TiO}_{2}-\mathrm{B}$ in a multilayer structure. $\mathrm{Ca}: \mathrm{TiO}_{2}-\mathrm{B}$ seems to be a more stable phase than $\mathrm{TiO}_{2}-\mathrm{B}$, and hence positioning the two phases in an alternating manner layer by layer may lead to counter-balance of unrelaxed strain and eventually a thicker film. Depending on the purpose, the thicknesses of respective layers could vary.

Thick films may be desired in certain device application or in the effort to scale up production. The multilayer structure may also be of interest for studies on interfacial effects.

\subsubsection{Band structure analysis}

One of the most important steps in investigating a new material for electrical applications is to understand its electronic band structure. Theoretical calculations are very instructive in this area. Prof. Emmanouil Kioupakis's group has calculated the band structure of the $\mathrm{TiO}_{2}-\mathrm{B}$ phase, revealing an indirect fundamental gap of $4.7 \pm 0.1 \mathrm{eV}$. The band gap for $\mathrm{Ca}: \mathrm{TiO}_{2}-\mathrm{B}$ is expected to be $\sim 0.3-0.5 \mathrm{eV}$ lower than that of $\mathrm{TiO}_{2}-\mathrm{B}$ due to the incorporation of $\mathrm{Ca}$, while the complete band structure will take a longer time to calculate as the structure is much more complicated. Some of the preliminary results are shown in Appendix A.

Such high band gaps poses particular challenges to experimental study. An attempt on exciting photoluminescence in both $\mathrm{Ca}: \mathrm{TiO}_{2}-\mathrm{B}$ and $\mathrm{TiO}_{2}-\mathrm{B}$ using a $325 \mathrm{~nm} \mathrm{He}-\mathrm{Cd}$ laser did not produce any strong band edge emissions, confirming that the optical band gap for both materials 
is higher than $3.8 \mathrm{eV}$. Lasers with wavelength shorter than $248 \mathrm{~nm}$ are usually gas lasers with high power (e.g. the $\mathrm{KrF}$ laser used in PLD) which would easily destroy the sample, so photoluminescence is not the suitable for this study. Shining deep UV light on the sample may result in spectrum response, however whether the luminescence is strong enough for the detector is yet unknown.

The possible solution is to utilize a synchrotron beam line, which is often employed in band structure analysis for high band gap materials. The collected data can be compared with, or guide theoretical calculations.

\subsubsection{Increasing electrical conductivity}

The poor electrical conductivity of the bronze materials is the major roadblock in improving their battery performance, causing low current collection efficiency and capacity loss. Hall measurements revealed that the resistivity of both $\mathrm{Ca}: \mathrm{TiO}_{2}-\mathrm{B}$ and $\mathrm{TiO}_{2}-\mathrm{B}$ is on the scale of $10^{4}-10^{5} \Omega \mathrm{cm}$ with carrier concentration of $10^{10}-10^{11} \mathrm{~cm}^{-3}$, as listed in Table 7.1. It should be noted that for such highly resistive films, the experimental error may be larger than usual. One mysterious fact is the change of carrier type between the two phases. It is likely that both $n$ - and $p$-type carrier groups exist in the bronze structures, and the measurements present the averaged effects resulting from the competition among these groups. Magneto-transport investigation which involves varying temperature and magnetic field is powerful in clarifying such characteristics, but it often requires higher carrier concentration and conductivity to be accurate.

As a common problem in metal oxide battery materials, the prevailing solution is to reduce the particle size for better contact with the current collector (e.g. carbon powder), or to encompass the particles with a conductive coating. Another more direct solution, which is also more difficult to achieve, is to increase the intrinsic conductivity of the material itself.

Doping is the most effective method to increase electron concentration or even lower the band gap by introducing defect or impurity energy levels. As unique phases difficult to synthesize in the first place, doping into $\mathrm{Ca}: \mathrm{TiO}_{2}-\mathrm{B}$ and $\mathrm{TiO}_{2}-\mathrm{B}$ while maintaining their lattice 
structures will not be easy. Theoretical calculations may again provide valuable directions. Dr. Baihai Li's DFT calculations have predicted a possible $\mathrm{TiPtO}_{4}$ phase, which has essentially the same structure as $\mathrm{TiO}_{2}$-B but with half of the Ti ions replaced by Pt ions. If such a new phase can be indeed synthesized, it should have a different band structure from that of $\mathrm{TiO}_{2}-\mathrm{B}$, and potentially a higher electrical conductivity.

\subsubsection{Origin of the pseudocapacitance}

The single crystals of $\mathrm{TiO}_{2}-\mathrm{B}$ and $\mathrm{Ca}: \mathrm{TiO}_{2}-\mathrm{B}$ offers a new opportunity to formulate an experimentally validated theory of nano-thermodynamics. The sloping regions in voltage at the end of charge and discharge (Figure 5.5(a)) are frequently seen in the literature for $\mathrm{TiO}_{2}-\mathrm{B}$ materials. Although it is often attributed to a pseudocapacitive process, the respective roles of surface and bulk are largely unknown. Since the majority of the published work is focused on various nanostructures where large surface area is an advantage, while the thin films discussed in this report are less than $200 \mathrm{~nm}$ thick, it is probable that certain surface capacitive effects contribute to the voltage behavior as well as to the measured capacity.

Having thus demonstrated this waterless synthesis of the bronze materials with high crystalline quality, we are for the first time equipping the field with a method to isolate surface effects from bulk effects. A systematic study of electrochemical properties on phase pure films ranging from a few monolayers to $\mu \mathrm{m}$ thickness would reveal the differences between regimes of surface-dominant versus bulk-dominant, clarifying the nature and origin of pseudocapacitance.

\subsubsection{Divalent ion batteries}

A few measures can be taken in further improving the performance of the batteries containing titania-bronze or understanding the electrochemical process. The full-cell prototypes discussed in Chapter 6 used cathode materials that had much larger mass than the anode. Matching the active material mass in the two electrodes will provide more information on the battery reactions, while reducing the total cell weight in real-life applications. Pairing the 
polycrystalline $\mathrm{Ca}: \mathrm{TiO}_{2}-\mathrm{B}$ films with optimized fast cathode materials such as coated $\mathrm{LiFePO}_{4}{ }^{15}$ will create a battery with ultrahigh rate capabilities. If a thin film cathode with high capacities is used, a solid state battery can be made with a solid electrolyte.

The bronze structures may be open enough for other ion species than $\mathrm{Li}^{+}$alone. Cycling the battery with $\mathrm{Na}^{+}$instead of $\mathrm{Li}^{+}$will enable researchers to track the heavier $\mathrm{Na}$ ions and observe the charge/discharge process with better clarity in techniques such as TEM and EELS. Furthermore, examining the possibility of a divalent ion battery is very interesting, as the open channels in the bronze crystals may allow $2+$ ions to pass with considerable mobility, such as $\mathrm{Mg}^{2+}$ and $\mathrm{Ca}^{2+}$. Although it is known that the electrochemistry is different for $2+$ ions, ${ }^{144}$ the $\mathrm{Ca}: \mathrm{TiO}_{2}-\mathrm{B}$ phase, already containing $\mathrm{Ca}$ ions, is a promising candidate for $\mathrm{Ca}^{2+}$ migration through its matrix.

\subsubsection{Related new phases}

In the attempt to modify the bronze structures, we have observed the formation of other novel phases as well. A single-crystalline (230) $\mathrm{MgTi}_{2} \mathrm{O}_{5}$ film was grown on (001) $\mathrm{SrTiO}_{3}$, while its crystal orientation rotates when deposited on (110) $\mathrm{SrTiO}_{3}$, resulting in a (200) film. This material is interesting for various properties, ${ }^{145}$ including increased compressibility ${ }^{146}$ and lithium reactivity. ${ }^{147}$

During thermal treatment of a Pt doped $\mathrm{CaTiO}_{3}$ thin film, small grains of a $\mathrm{CaTi}_{2} \mathrm{O}_{5}$ phase were observed in TEM, along with $\mathrm{Ca}: \mathrm{TiO}_{2}-\mathrm{B}$ grains under different redox conditions. It seems to have the same lattice structure as $\mathrm{MgTi}_{2} \mathrm{O}_{5}$, but with the $\mathrm{Mg}$ ions replace by $\mathrm{Ca}$ ions.

To the best of my knowledge, this is the first synthesis of single-crystalline $\mathrm{MgTi}_{2} \mathrm{O}_{5}$ thin films, while the $\mathrm{CaTi}_{2} \mathrm{O}_{5}$ phase has not be reported scientifically, other than the only reference in a patent application. ${ }^{13}$ The growth optimization, band structure study, property testing and electrochemical reactivity including in possible $2+$ ion batteries of these novel phases are brand new topics of research interests. 
Table 7.1 Electrical properties of a (001) $\mathrm{Ca}: \mathrm{TiO}_{2}-\mathrm{B}$ film and a (001) $\mathrm{TiO}_{2}-\mathrm{B} / \mathrm{Ca}: \mathrm{TiO}_{2}-\mathrm{B}$ dual layer film both grown on (001) $\mathrm{SrTiO}_{3}$ substrates at $800{ }^{\circ} \mathrm{C}$.

\begin{tabular}{lcccc}
\hline Film & Thickness $(\mathrm{nm})$ & $\rho(\Omega \mathrm{cm})$ & $n\left(\mathrm{~cm}^{-3}\right)$ & Type \\
\hline $\mathrm{Ca}: \mathrm{TiO}_{2}-\mathrm{B}$ & 65 & $8.89 \times 10^{4}$ & $5.77 \times 10^{10}$ & $n$ \\
$\mathrm{TiO}_{2}-\mathrm{B} / \mathrm{Ca}: \mathrm{TiO}_{2}-\mathrm{B}$ & $25 / 20$ & $5.99 \times 10^{4}$ & $2.18 \times 10^{10}$ & $p$ \\
\hline
\end{tabular}




\section{Appendix A \\ $\mathrm{TiO}_{2}-\mathrm{B}$ Band Structure by First Principles Calculations}

The band structure of the $\mathrm{TiO}_{2}-\mathrm{B}$ phase has been calculated by Dylan Bayerl in Prof. Emmanouil Kioupakis's group. The band structure calculations of the $\mathrm{Ca}: \mathrm{TiO}_{2}-\mathrm{B}$ phase is more complicated and still underway.

The first principles methodology is based on density functional theory (DFT) and many-body perturbation theory in the GW approximation. DFT provides single-particle states and eigenenergies to which we apply quasiparticle corrections from the GW method to obtain predictions of electronic band gaps. The DFT $+\mathrm{GW}$ methodology predicts band gaps in very good agreement with experiment for most material systems, while the precision of our calculations is estimated at $\pm 0.1 \mathrm{eV}$. Our DFT $+\mathrm{GW}$ calculations allow us to interpolate the electronic band structure using maximally-localized Wannier functions, yielding high-resolution band structures from first-principles.

The band structure calculation of the $\mathrm{TiO}_{2}-\mathrm{B}$ polymorph (Figure 1) reveals an indirect fundamental gap of $4.7 \mathrm{eV}( \pm 0.1 \mathrm{eV})$. The fundamental transition is between the conduction band minimum at $\Gamma$ and low-symmetry valence band maxima near the N-point. Analysis of atomic orbital projections confirms that conduction band edge states are primarily of titanium d-orbital character, whereas valence band edge states have predominantly oxygen p-orbital character. For $\mathrm{Ca}: \mathrm{TiO}_{2}-\mathrm{B}\left(\mathrm{CaTi}_{5} \mathrm{O}_{11}\right)$, which is essentially a twinned, augmented variant of $\mathrm{TiO}_{2}-\mathrm{B}$ with an extra Ti-Ca interleaved layer, preliminary theoretical investigation of the calcium-enriched $\mathrm{TiO}_{2}-\mathrm{B}$ phase suggests that calcium incorporation slightly reduces the band gap of the bronze polymorph by 0.3 to $0.5 \mathrm{eV}$. We speculate that the gap is reduced by a combination of Brillouin zone folding 
from breaking crystalline symmetry and introduction of near-edge calcium levels, though the exact mechanism has not yet been identified. 

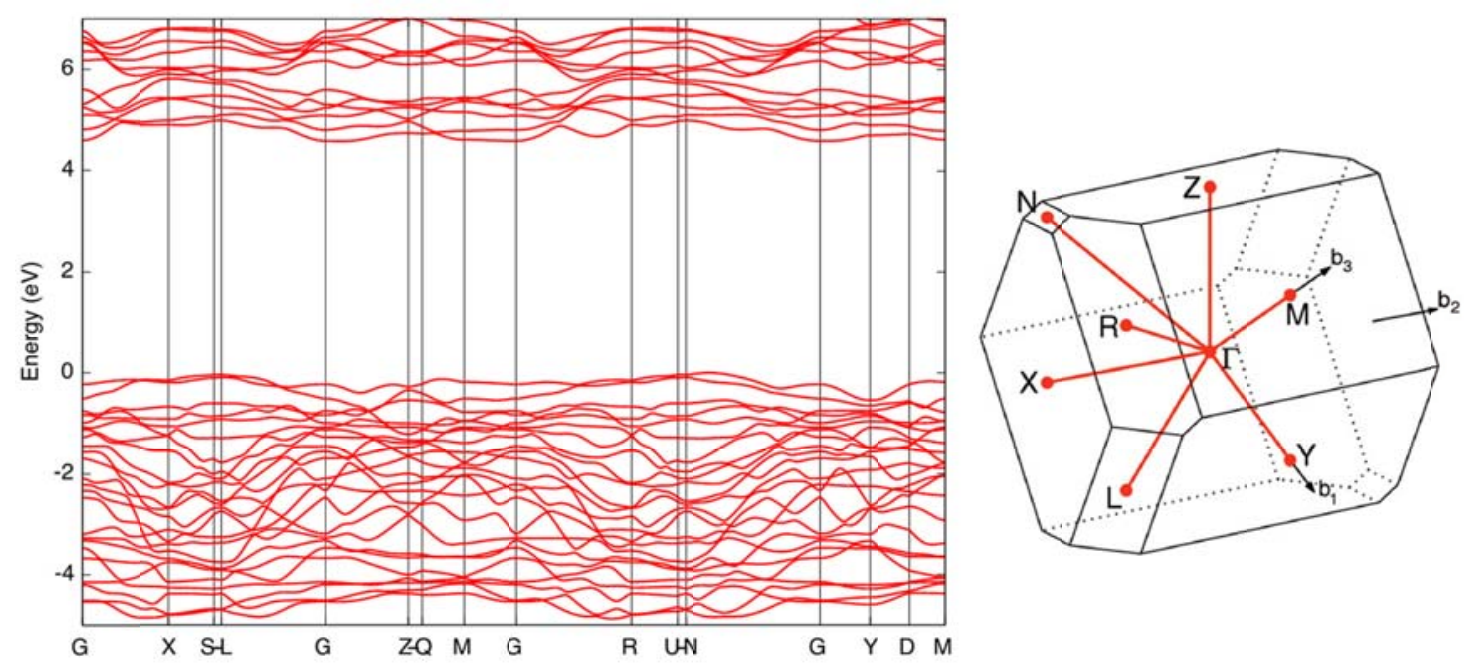

Figure A.1 (Left) band structure of the $\mathrm{TiO}_{2}-\mathrm{B}$ polymorph with valence band maximum located at $0 \mathrm{eV}$. (Right) schematic of the Brillouin zone with several high-symmetry points labeled. [Figure courtesy: Dylan Bayerl and Prof. Emmanouil Kioupakis] 


\section{Appendix B}

\section{Enhanced Thermoelectric Properties in a Metal-Perovskite System}

This appendix is intended to document a separate project performed during the PhD studies. Although the topic is different from the focus of this thesis, the experimental methods are largely the same as detailed in Chapter 2.

\section{B.1 Introduction}

Precious metal nanoparticles precipitating from and dissolving into perovskite structures in redox conditions opened up new opportunities for catalysis applications. It is also well-known that nano-inclusions may help enhance the figure of merit in thermoelectric materials by reducing the thermal conductivity. However, these inclusions, in many cases, create scattering centers that reduce electron mobility and electrical conductivity. Here we demonstrate the first experimental proof that $\mathrm{Pt}$ nano-inclusions can precipitate in heavily $\mathrm{Nb}$-doped $\mathrm{SrTiO}_{3}$ thin films with a single step of deposition, presenting ultrahigh electrical conductivity on the order of $10^{4}$ $\mathrm{S} / \mathrm{cm}$, while in the meantime reducing the thermal conductivity, resulting in a significant enhancement of the figure of merit. Beyond thermoelectrics, the $\mathrm{Pt}-\mathrm{SrTiO}_{3}$ system may also be of interest for photocatalytic applications.

Oxide materials are attractive for thermoelectric applications due to their stability at elevated temperatures, which is especially valuable in high temperature environment such as exhaust gases and industrial catalytic reactors. The figure of merit which gauges the energy conversion efficiency, defined as $Z T=T\left(\sigma S^{2}\right) / \kappa$, where $T$ is the absolute temperature, $\sigma$ is the 
electrical conductivity, $S$ is the Seebeck coefficient (also known as the thermopower), and $\kappa$ is the thermal conductivity, is difficult to enhance in many material systems as the effects are often intertwined. Increasing carrier concentration by doping usually leads to an increase in electrical conductivity and a decrease in mobility $(\mu)$, characterized by $\sigma=e n \mu$, where $e$ is the elementary charge, while in the meantime lowering the thermopower, expressed by $S=\left[\left(8 \pi^{2} k_{\mathrm{B}}{ }^{2}\right) / 3 e h^{2}\right] m^{*} T(\pi / 3 n)^{2 / 3}$ for metals or degenerate semiconductors (parabolic band, energy-independent scattering approximation), ${ }^{148}$ where $k_{\mathrm{B}}$ is the Boltzmann constant, $h$ is the Planck constant, $m^{*}$ is the effective mass of the charge carrier and $n$ is the carrier concentration. Therefore, the effort to improve $Z T$ is often two-fold: decreasing the thermal conductivity $\kappa$, or maximizing the power factor defined as $P F=\sigma S^{2}$, which is often a compromise between $\sigma$ and $S$, since higher carrier concentration typically leads to higher electrical conductivity but lower Seebeck coefficient.

Precious metal movements into and out of perovskite oxides between a solid solution and metallic nanoparticles under oxidizing and reducing conditions, respectively, have been proposed as self-generating catalysts, which were demonstrated in various material systems including Pd-LaFeO 3, Rh-CaTiO 3 and Pt-CaTiO ${ }_{3}{ }^{149-152}$ Besides their applications in automotive emissions control and photocatalytic hydrogen production, ${ }^{153}$ nano-inclusions serving as phonon-scattering centers in a host matrix may also be utilized to lower the thermal conductivity of bulk thermoelectric materials, ${ }^{154,155}$ thus increasing $Z T$. The challenge in such an approach is to avoid electron-scattering by the nano-inclusions which leads to decreasing electrical conductivity. In this Appendix, we report that Pt nanoparticles precipitating in $\mathrm{Nb}$-doped $\mathrm{SrTiO}_{3}(\mathrm{STO})$ thin films grown by pulsed laser deposition (PLD) can reduce the thermal conductivity by $\sim 20 \%$. Further increasing the carrier concentration by heavy $\mathrm{Nb}$ doping and adding oxygen vacancies by switching the growth atmosphere to Ar result in an ultrahigh electrical conductivity of $1.62 \times 10^{4}$ $\mathrm{S} / \mathrm{cm}$, and a power factor of $1.25 \mathrm{~mW} \mathrm{~K}^{-2} \mathrm{~m}^{-1}$ at $300 \mathrm{~K}$, which exhibits a $350 \%$ increase over the film grown in $\mathrm{O}_{2}$ with no $\mathrm{Pt}$ nano-inclusions. 


\section{B.2 Experimental methods}

The PLD targets were constructed by mixing $\mathrm{SrTiO}_{3}, \mathrm{Nb}_{2} \mathrm{O}_{5}$ and $\mathrm{PtO}_{2}$ powders with specific mole percentage of metal ions, sintering at $1400{ }^{\circ} \mathrm{C}$ and cold pressing into dense pellets. In the $\mathrm{STO}$ host, both $\mathrm{Nb}$ and $\mathrm{Pt}$ substitute $\mathrm{Ti}$ ions, and thus the general chemical formula for the series of thin films discussed in this Appendix can be expressed as $\operatorname{SrTi}_{1-x-y} \mathrm{Nb}_{x} \mathrm{Pt}_{y} \mathrm{O}_{3}$ (STNPO). The PLD vacuum chamber has a base pressure $<3.8 \times 10^{-9}$ Torr. A $248 \mathrm{KrF}$ excimer laser with a pulse duration of $22 \mathrm{~ns}$ was used for the deposition at a repetition rate of $5-10 \mathrm{~Hz}$, and the substrate-target distance was set to $6.35 \mathrm{~cm}$. Purified $\mathrm{O}_{2}$ or $\mathrm{Ar}$ gas was backfilled into the chamber to a particular partial pressure during growth, which was maintained in the cooling process to room temperature (RT). The films studied in this work have thicknesses of 100-200nm, which were measured by a Veeco Dektak profilometer and confirmed with transmission electron microscopy (TEM) images. Microstructures of the films were studied with a Rigaku rotating anode diffractometer using $\mathrm{Cu} \mathrm{K} \alpha$ radiation, and a JEOL 2100F TEM equipped with a spherical aberration corrector. Electronic transport properties were measured using SQUID (superconducting quantum interference device) in Hall-bar configuration. Thermal conductivity was measured at $300 \mathrm{~K}$ by employing the $3 \omega$ method, ${ }^{156}$ which requires that the substrate has a significantly higher thermal conductivity than that of the film under investigation for accurate measurement, so the STNPO films discussed in this study were grown on Si (100) substrates with a 20 unit-cell STO buffer layer. ${ }^{157}$ Since most of the Nb-doped STO films are highly conductive, which is invalid for the $3 \omega$ method, each sample had a replica grown at identical conditions but with an additional insulating STO layer $\left(700{ }^{\circ} \mathrm{C}, 50\right.$ mTorr $\left.\mathrm{O}_{2}\right) \sim 10 \mathrm{~nm}$ thick on top for the thermal conductivity measurement.

\section{B.3 Results and discussion}

A $\mathrm{SrTi}_{0.75} \mathrm{Nb}_{0.20} \mathrm{Pt}_{0.05} \mathrm{O}_{3}$ film was first grown at $800{ }^{\circ} \mathrm{C}$ in 50 mTorr of $\mathrm{O}_{2}$. The $\sim 8 \mathrm{~nm}$ STO buffer layer on the Si substrate absorbs most of the mismatch strains, benefiting the 
homoepitaxial growth of the STNPO thin film on top. As a result, the film was very uniform with a smooth surface. The $\theta-2 \theta \mathrm{X}$-ray diffraction (XRD) patterns (not shown) confirmed that the STNPO film is single crystalline, (001) oriented, without any impurity peaks detected. Fitting the $00 l$ family peak positions to the Bragg's law yields a lattice constant of $a=3.961 \AA$, a $1.4 \%$ increase from that of the pure STO (3.905 $\AA$ ), as the $\mathrm{Nb}$ and Pt ions which have larger ionic radii than $\mathrm{Ti}^{4+}$ expand the lattice. No Pt nanoparticles were observed in the as-grown film, indicating that $\mathrm{Pt}$ is well dispersed in the STO matrix. Hall measurement reveals that the film is $n$-type but quite resistive, exhibiting a carrier concentration of $n=1.49 \times 10^{10} \mathrm{~cm}^{-3}$ and an electrical conductivity of $\sigma=8.19 \times 10^{-6} \mathrm{~S} / \mathrm{cm}$ at RT, which is very low for a $20 \% \mathrm{Nb}$ doped STO film. ${ }^{158}$ This is because the film was grown in an oxidizing condition, while significant changes were obtained through thermal treatment. After annealing in $10 \% \mathrm{H}_{2}\left(\right.$ in $\left.\mathrm{N}_{2}\right)$ at $800{ }^{\circ} \mathrm{C}$ for $40 \mathrm{~min}$, the RT carrier concentration and electrical conductivity have increased by several orders of magnitude to $n=3.86 \times 10^{19} \mathrm{~cm}^{-3}$ and $\sigma=6.30 \mathrm{~S} / \mathrm{cm}$, respectively, due to the generation of oxygen vacancies in the reduction process. No metallic Pt particles were observed in the reduced film. The thermal treatment did not have an apparent influence on the thermal conductivity, which was $4.60 \pm 0.44 \mathrm{~W} \mathrm{~m}^{-1} \mathrm{~K}^{-1}$ at RT [measured by Dr. Gun-Ho Kim in Prof. Kevin Pipe's group at Department of Mechanical Engineering]. One may notice that such a value is much lower than the thermal conductivity of bulk STO $\left(11.2 \mathrm{~W} \mathrm{~m}^{-1} \mathrm{~K}^{-1}\right)$, which has been previously attributed to the relatively higher defect density in STO films grown by PLD using ceramic targets. ${ }^{159}$

The absence of metallic Pt precipitation in the reduced STNPO film may be because the reducing condition was inadequate for the Pt-STO system, ${ }^{150}$ or that the film surface was effectively shielding out the reduction gas from reacting with the bulk of the film. To change the redox environment as the film is being deposited, the $\mathrm{O}_{2}$ partial pressure during growth was lowered substantially, and the effect was immediate. Figure B.1(a) shows the high-angle annular dark-field (HAADF) STEM image of a $\mathrm{SrTi}_{0.75} \mathrm{Nb}_{0.20} \mathrm{Pt}_{0.05} \mathrm{O}_{3}$ film grown in 0.01 mTorr $\mathrm{O}_{2}$ instead of 50 mTorr (other conditions fixed), where Pt particles generally 1-3 nm in diameter are uniformly distributed throughout the film, many of which are exposed on the surface, as shown 
in Figure B.1(c). The face-centered cubic (fcc, $a=3.924 \AA$ ) structure of the Pt nanoparticle is clearly seen (Figure B.1(b)), which manifests itself as a near-perfect epitaxial cluster embedded in STO due to the close lattice parameters, imposing no obvious strain on the host matrix. The low oxygen atmosphere has also enhanced the electrical properties to $n=3.30 \times 10^{20} \mathrm{~cm}^{-3}$ and $\sigma=77.7 \mathrm{~S} / \mathrm{cm}$, both another order of magnitude higher than those of the previous film grown in 50 mTorr $\mathrm{O}_{2}$ and reduced after, suggesting that pushing the growth conditions in the reducing direction is more effective than post-growth reduction treatment. Further annealing of this film in $10 \% \mathrm{H}_{2}$ for $2 \mathrm{hr}$ had no significant effects on either its electrical properties or the morphology of the Pt nanoparticles. As expected, these metallic nano-inclusions have reduced the thermal conductivity of the STNPO film by $20 \%$, to $3.66 \pm 0.20 \mathrm{~W} \mathrm{~m}^{-1} \mathrm{~K}^{-1}$. Though proposed from earlier calculations, ${ }^{151}$ this is, to the best of our knowledge, the first experimental proof that Pt particles may form out of a STO perovskite host.

In order to further improve the electrical conductivity, two different approaches were taken: increasing the $\mathrm{Nb}$-doping, and introducing more oxygen vacancies by growing the films in Ar gas instead of $\mathrm{O}_{2}$. Figure B.2(a) shows the comparison of carrier concentration in STNPO films grown under different combinations of $\mathrm{Nb}$ doping level $(20 \%$ or $30 \%)$ and growth atmosphere (0.01 mTorr $\mathrm{O}_{2}$ or 20 mTorr Ar). All four films contained 5\% Pt, which has precipitated as metallic particles embedded in the STO matrix. It is clear that both approaches have increased the carrier concentration considerably. With $30 \% \mathrm{Nb}$ doping and Ar growth, the carrier concentration is $1.71 \times 10^{22} \mathrm{~cm}^{-3}$ at $10 \mathrm{~K}$ and $1.89 \times 10^{22} \mathrm{~cm}^{-3}$ at $300 \mathrm{~K}$, resulting in an exceedingly high electrical conductivity of $1.62 \times 10^{4} \mathrm{~S} / \mathrm{cm}$ at $300 \mathrm{~K}$, as shown in Figure B.2(b). Among the four samples, only the film with $20 \% \mathrm{Nb}$ grown in $\mathrm{O}_{2}$ exhibits semi-conductive behavior, while the other 3 all show metallic behavior.

Besides the electrical properties, the deposition atmosphere also influences the morphology of the Pt nanoparticles. In some areas of a $\mathrm{SrTi}_{0.65} \mathrm{Nb}_{0.30} \mathrm{Pt}_{0.05} \mathrm{O}_{3}$ film grown in 0.01 mTorr $\mathrm{O}_{2}$, the Pt nanoparticles may extend along the growth direction, forming nanorods instead of round particles, an example of which is shown in Figure B.3(a). Energy dispersive 
spectroscopy (EDS) results corroborate that these nano-inclusions are indeed Pt (Figure B.3(b)), ruling out the possibility of metallic $\mathrm{Nb}$ precipitation in a heavily doped film. When the deposition atmosphere is switched to 20 mTorr Ar, these nanorods essentially extend over almost the entire thickness of the film and reach the surface, as shown in Figure B.3(c), where the Pt rods are 1-2 $\mathrm{nm}$ wide and $>110 \mathrm{~nm}$ long. A close examination at the interface revealed that the nanorods are not in direct contact with the substrate, but $\sim 10 \mathrm{~nm}$ away from its surface. Within that area, small round-shaped Pt particles can still be identified, suggesting that these nanorods may be growing on the basis of nucleation cores along with the film. Since the films grown in Ar contain significantly more oxygen vacancies than the ones grown in $\mathrm{O}_{2}$, it is plausible that the stretching effect of the $\mathrm{Pt}$ particles/rods is linked to the higher concentration of oxygen vacancies, which are essentially crystal defects in the STO host matrix that allow foreign metallic clusters to expand over the course of growth.

To investigate the effects which the Pt nano-inclusions may impose on the carrier transport properties in the STNPO films, a $\mathrm{SrTi}_{0.70} \mathrm{Nb}_{0.30} \mathrm{O}_{3}$ (STNO) film was grown in 20 mTorr Ar, with no Pt doping during PLD target fabrication. As expected, the STNO film is uniform and in high crystal quality, without any nanoparticles forming internally. The transport properties of this film are then compared with the $\mathrm{SrTi}_{0.65} \mathrm{Nb}_{0.30} \mathrm{Pt}_{0.05} \mathrm{O}_{3}$ film grown under the same conditions over a temperature range of 3-300 $\mathrm{K}$, as shown in Figure B.4. Both films have ultrahigh electrical conductivity on the order of $10^{4} \mathrm{~S} / \mathrm{cm}$, which is slightly higher in the STNO film than in the STNPO film. The difference is larger at low temperature, where electron scattering on the boundaries between the STO matrix and the metallic Pt clusters dominates the conduction characteristics. As the temperature increases, inelastic electron-phonon scattering caused by lattice thermal vibration gradually takes dominance, overwhelming the influence from the $\mathrm{Pt}$ nano-inclusions, resulting in very close values of electrical conductivity in the two films above $250 \mathrm{~K} .{ }^{160}$ Having Pt or not, the Hall mobility is almost the same in the two films over the entire tested temperature range, indicating that electron scattering at Pt-STO boundaries is elastic in nature without noticeable energy loss, and that the potential barrier between the Fermi levels of 
the heavily Nb-doped STO and the Pt metal is high enough to prevent a significant amount of electron from entering or being trapped in the Pt nano-inclusions. ${ }^{161}$ This is also evidenced by the fact that the carrier concentration in the STNPO film is $<4 \%$ lower than that in the STNO film, and the difference is only larger at low temperature, when the electrons have less kinetic energy and are easier to be captured by trapping centers such as crystal defects or dangling bonds surrounding the Pt nano-inclusions. The smaller carrier concentration has, however, contributed to an increased Seebeck coefficient (absolute value, $S \propto T n^{-2 / 3}$ ) in the STNPO film, where the difference is greater at higher temperatures, as seen in Figure B.4(d).

The increase of the Seebeck coefficient is magnified in the power factor $\left(P F=\sigma S^{2}\right)$. As shown in Figure B.5, the $P F$ of the SPNO films, either grown in $\mathrm{Ar}$ or $\mathrm{O}_{2}$, is only slightly higher below $50 \mathrm{~K}$, due to the higher electrical conductivity at low temperature (Figure B.4(a)). With increasing temperature, the higher Seebeck coefficient consequently leads to higher $P F$ in the STNPO films containing Pt nano-inclusions grown in both ambient gases. Benefiting from the ultrahigh electrical conductivity, the $\mathrm{SrTi}_{0.65} \mathrm{Nb}_{0.30} \mathrm{Pt}_{0.05} \mathrm{O}_{3}$ film grown in Ar with Pt nanorods has a $P F=1.25 \mathrm{~mW} \mathrm{~K}^{-2} \mathrm{~m}^{-1}$ at $300 \mathrm{~K}$, a $3.5 \times$ enhancement comparing with the $\operatorname{SrTi}_{0.70} \mathrm{Nb}_{0.30} \mathrm{O}_{3}$ film grown in $\mathrm{O}_{2}$, and $Z T$ is estimated to be $\sim 0.10$. Considering the thermal stability of both the perovskite STO host and the Pt nano-inclusions, it is expected that as the temperature increases, the thermal conductivity decreases and the Seebeck coefficient further increases, ${ }^{162,163}$ and hence these STNPO films will exhibit significantly larger $Z T$ at higher temperatures.

\section{B.4 Conclusions}

Pt nanoparticles can precipitate in STO thin films under reducing conditions, which may elongate into nanorods when the film is grown in Ar atmosphere. The Pt nano-inclusions reduce the thermal conductivity of the thin films by $\sim 20 \%$, without substantially affecting electronic transport. $\mathrm{Nb}$ doping and adding oxygen vacancies enhance the electrical properties regardless of Pt precipitation, leading to a significant increase of the power factor. 

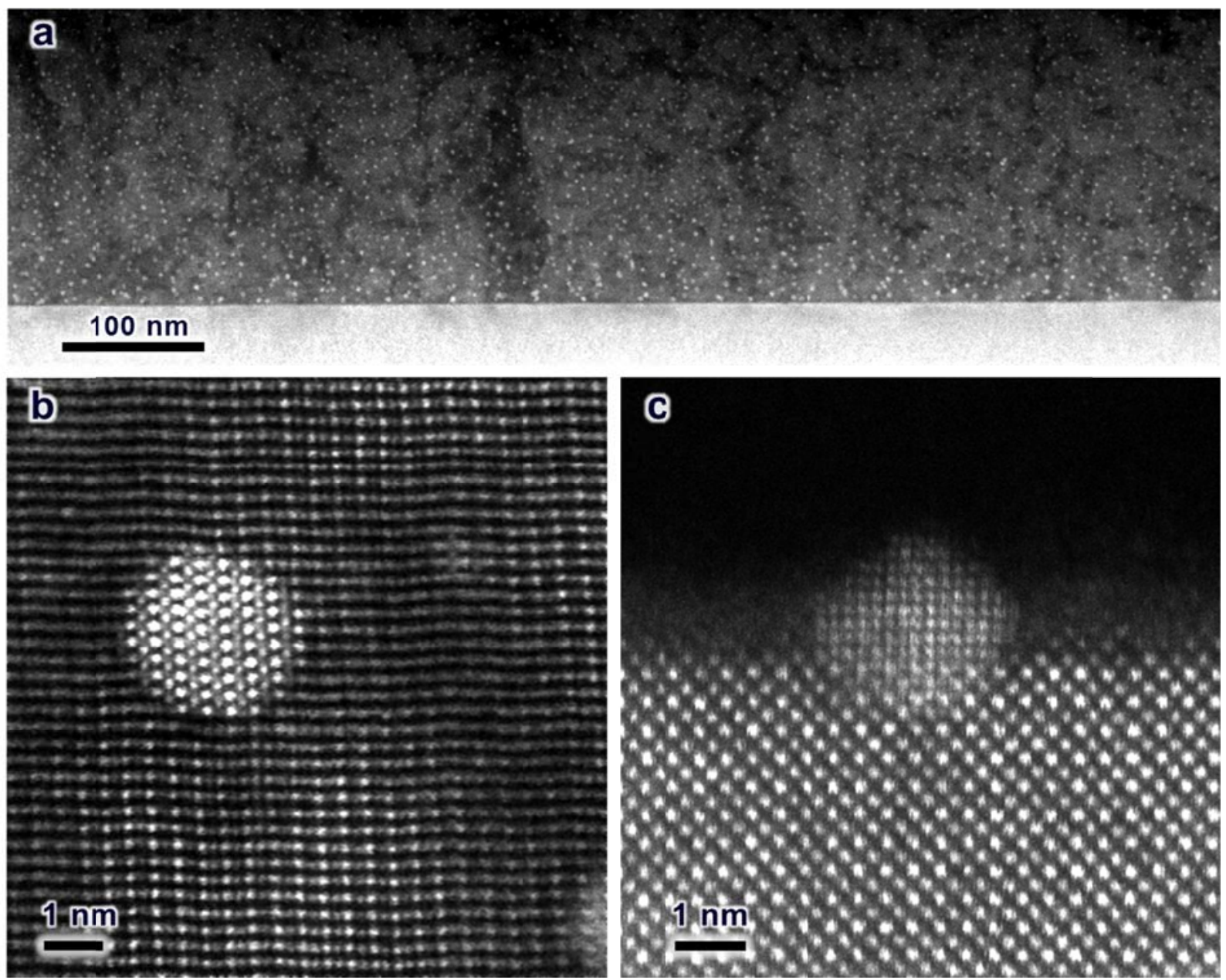

Figure B.1 a) STEM image of a $\mathrm{SrTi}_{0.75} \mathrm{Nb}_{0.20} \mathrm{Pt}_{0.05} \mathrm{O}_{3}$ film grown in $0.01 \mathrm{mT} \mathrm{O}_{2}$ at $800{ }^{\circ} \mathrm{C}$ containing Pt nanoparticles as-grown. b) High resolution HAADF STEM image showing one Pt particle with $\sim 2.5 \mathrm{~nm}$ diameter in the STO host. c) A typical Pt particle sitting on the surface of the film. [TEM image credit: Shuyi Zhang] 

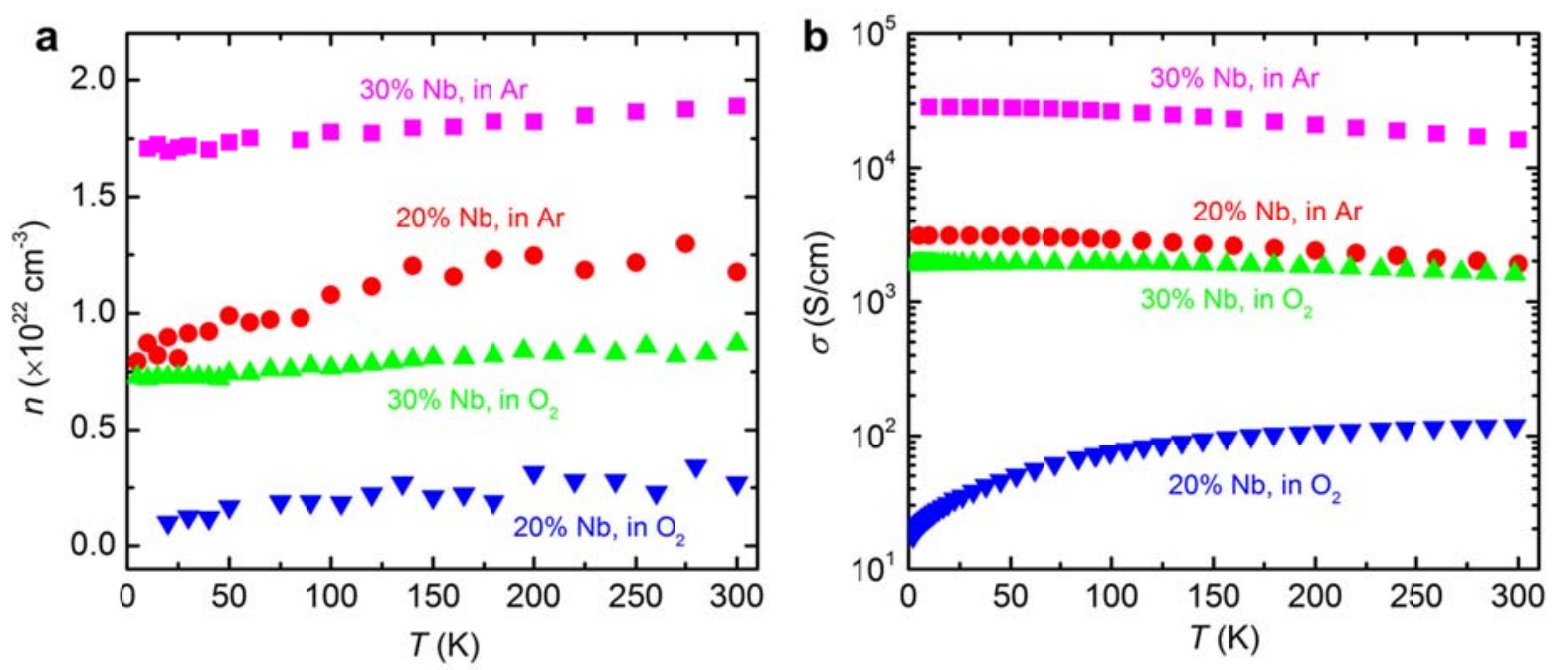

Figure B.2 Comparison of electrical properties in four STNPO films growing in either 20 mTorr Ar or $0.01 \mathrm{mTorr}_{2}$, all containing 5\% Pt which has precipitated as metallic nanoparticles: a) carrier concentration; b) electrical conductivity. [Temperature dependent transport data measured by Hang Chi in Prof. Ctirad Uher's group at Department of Physics] 


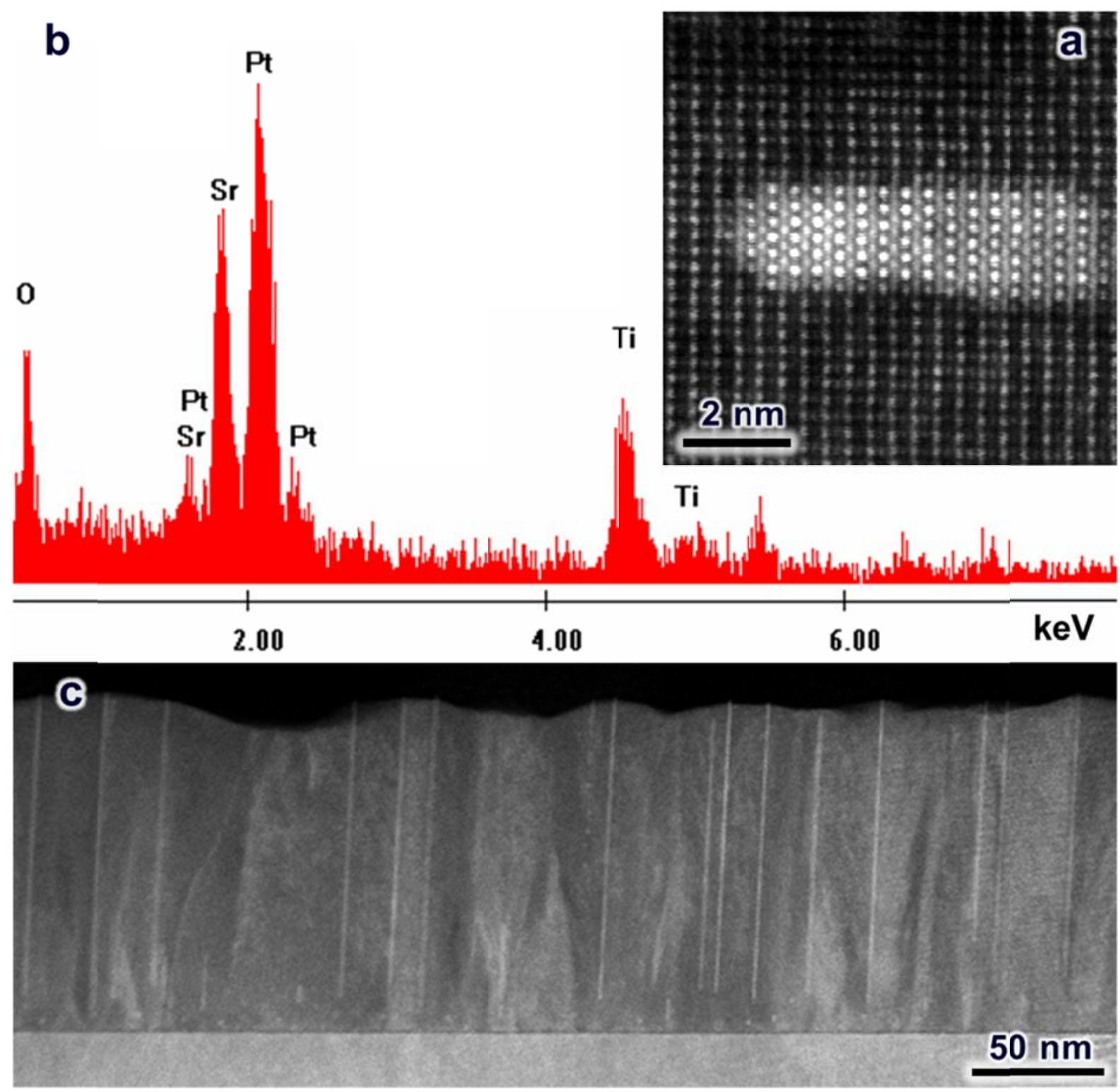

Figure B.3 a) HAADF STEM image of an elongated Pt nanoparticle. b) EDS spectrum taken as the beam was focused on the Pt particle. c) STEM image of a $\mathrm{SrTi}_{0.65} \mathrm{Nb}_{0.30} \mathrm{Pt}_{0.05} \mathrm{O}_{3}$ film grown in Ar atmosphere showing Pt nanorods. [TEM image credit: Shuyi Zhang \& Yi Zhang] 

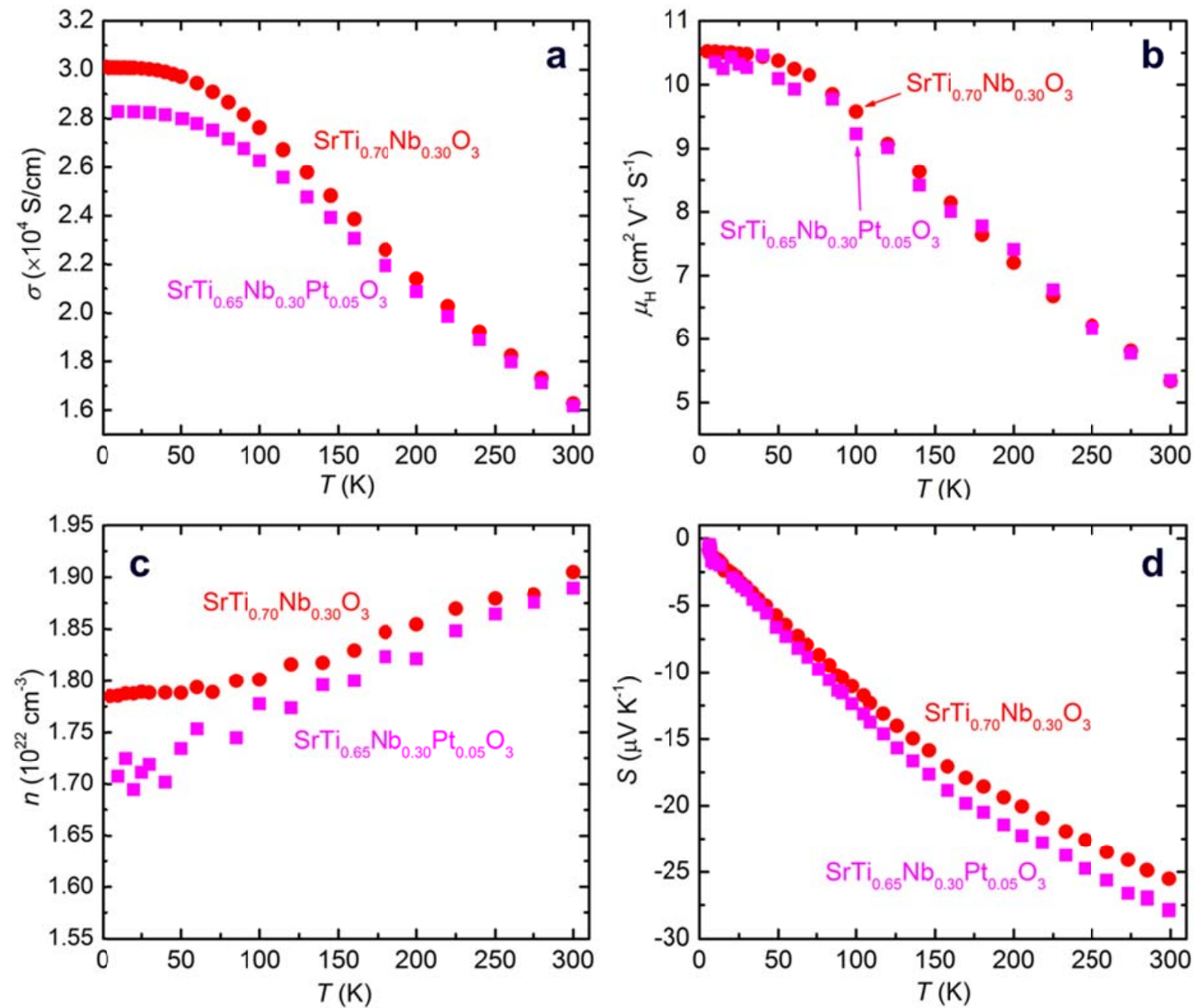

Figure B.4 Temperature dependent electronic transport properties in two $30 \% \mathrm{Nb}$ doped films grown in Ar atmosphere, with or without Pt nano-inclusions: a) electrical conductivity; b) Hall mobility; c) carrier concentration; d) Seebeck coefficient. [Temperature dependent transport data measured by Hang Chi in Prof. Ctirad Uher's group at Department of Physics] 


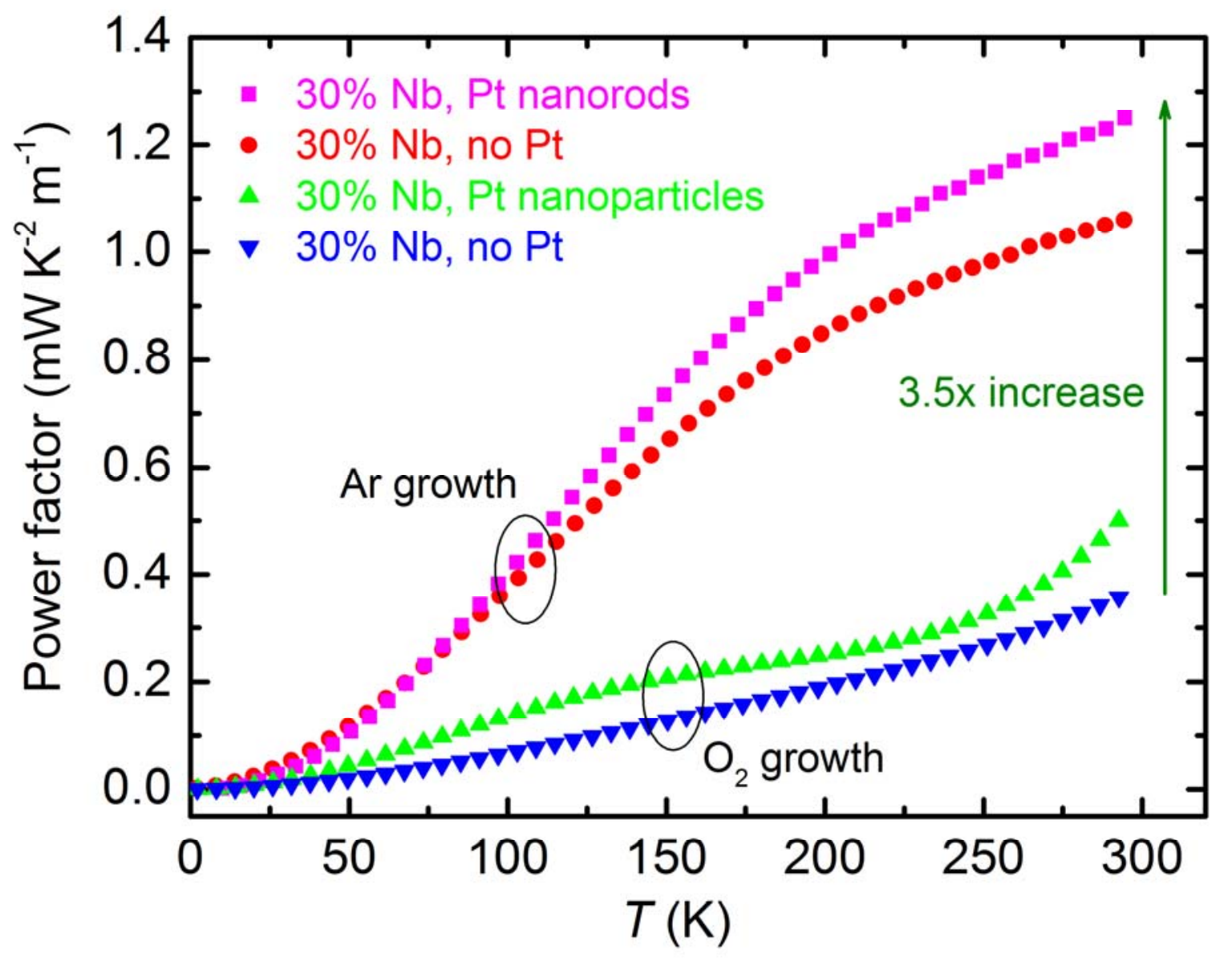

Figure B.5 Temperature dependent power factor in four 30\% Nb doped STNPO films. At $300 \mathrm{~K}$, the film containing Pt nanorods grown in Ar shows a 3.5 $\times$ increase of $P F$ from the film with no $\mathrm{Pt}$ grown in $\mathrm{O}_{2}$. 


\section{Bibliography}

${ }^{1}$ Fujishima, A.; Honda, K. Nature 1972, 238, 37.

${ }^{2}$ Oregan, B.; Gratzel, M. Nature 1991, 353, 737.

${ }^{3}$ Chen, X.; Mao, S. S. Chemical Reviews 2007, 107, 2891.

${ }^{4}$ Carp, O.; Huisman, C. L.; Reller, A. Prog. Solid State Chem. 2004, 32, 33.

${ }^{5}$ Kaspar, T. C.; Ney, A.; Mangham, A. N.; Heald, S. M.; Joly, Y.; Ney, V.; Wilhelm, F.; Rogalev, A.; Yakou, F.; Chambers, S. A. Phys. Rev. B 2012, 86.

${ }^{6}$ Banfield, J. F.; Veblen, D. R.; Smith, D. J. Am. Miner. 1991, 76, 343.

${ }^{7}$ Marchand, R.; Brohan, L.; Tournoux, M. Materials Research Bulletin 1980, 15, 1129.

${ }^{8}$ Feist, T. P.; Davies, P. K. Journal of Solid State Chemistry 1992, 101, 275.

9 J. P. Perdew, K. Burke, M. Ernzerhof, Phys. Rev. Lett. 1996, 77, 3865.

${ }^{10}$ G. Kresse, J. Hafner, Phys. Rev. B 1993, 48, 13115.

11 P. Deak, B. Aradi, T. Frauenheim, E. Janzen, A. Gali, Phys. Rev. B 2010, 81, 153203.

12 Zhang, K.; Katz, M. B.; Li, B.; Kim, S. J.; Du, X.; Hao, X.; Jokisaari, J. R.; Zhang, S.; Graham, G. W.; Van der Ven, A.; Bartlett, B. M.; Pan, X. Advanced Materials 2014, 26, 7365.

13 R. Ancora, M. Borsa, M. I. Marchi (Italcementi S.P.A), US20110192325 A1, 2011.

${ }^{14}$ Nishimura, S.-i.; Kobayashi, G.; Ohoyama, K.; Kanno, R.; Yashima, M.; Yamada, A. Nat Mater 2008, 7, 707.

15 Kang, B.; Ceder, G. Nature 2009, 458, 190.

${ }^{16}$ Zukalova, M.; Kalbac, M.; Kavan, L.; Exnar, I.; Graetzel, M. Chemistry of Materials 2005, 17, 1248.

17 Augustyn, V.; Come, J.; Lowe, M. A.; Kim, J. W.; Taberna, P. L.; Tolbert, S. H.; Abruna, H. D.; Simon, P.; Dunn, B. Nature Materials 2013, 12, 518. 
18 Arico, A. S.; Bruce, P.; Scrosati, B.; Tarascon, J. M.; Van Schalkwijk, W. Nature Materials 2005, 4, 366.

19 Kogure, T.; Umezawa, T.; Kotani, Y.; Matsuda, A.; Tatsumisago, M.; Minami, T. Journal of the American Ceramic Society 1999, 82, 3248.

20 Armstrong, A. R.; Armstrong, G.; Canales, J.; Bruce, P. G. Angewandte Chemie International Edition 2004, 43, 2286.

${ }^{21}$ Armstrong, G.; Armstrong, A. R.; Canales, J.; Bruce, P. G. Chemical Communications 2005, 2454.

22 Beuvier, T.; Richard-Plouet, M.; Mancini-Le Granvalet, M.; Brousse, T.; Crosnier, O.; Brohan, L. Inorganic Chemistry 2010, 49, 8457.

${ }^{23}$ Liu, H.; Bi, Z.; Sun, X.-G.; Unocic, R. R.; Paranthaman, M. P.; Dai, S.; Brown, G. M. Advanced Materials 2011, 23, 3450.

${ }^{24}$ Liu, S.; Jia, H.; Han, L.; Wang, J.; Gao, P.; Xu, D.; Yang, J.; Che, S. Advanced Materials 2012, 24,3201 .

${ }^{25}$ Zhou, W. J.; Gai, L. G.; Hu, P. G.; Cui, J. J.; Liu, X. Y.; Wang, D. Z.; Li, G. H.; Jiang, H. D.; Liu, D.; Liu, H.; Wang, J. Y. Crystengcomm 2011, 13, 6643.

${ }^{26}$ Etacheri, V.; Kuo, Y.; Van der Ven, A.; Bartlett, B. M. Journal of Materials Chemistry A 2013, $1,12028$.

${ }^{27}$ Yang, Z. G.; Choi, D.; Kerisit, S.; Rosso, K. M.; Wang, D. H.; Zhang, J.; Graff, G.; Liu, J. Journal of Power Sources 2009, 192, 588.

28 Armstrong, G.; Armstrong, A. R.; Bruce, P. G.; Reale, P.; Scrosati, B. Advanced Materials 2006, 18, 2597.

${ }^{29}$ Chen, J. S.; Tan, Y. L.; Li, C. M.; Cheah, Y. L.; Luan, D. Y.; Madhavi, S.; Boey, F. Y. C.; Archer, L. A.; Lou, X. W. J. Am. Chem. Soc. 2010, 132, 6124.

${ }^{30}$ Chen, J. S.; Lou, X. W. Journal of Power Sources 2010, 195, 2905.

${ }^{31}$ Feckl, J. M.; Fominykh, K.; Doblinger, M.; Fattakhova-Rohlfing, D.; Bein, T. Angewandte Chemie-International Edition 2012, 51, 7459.

${ }^{32}$ Hao, X. G.; Bartlett, B. M. Advanced Energy Materials 2013, 3, 753.

33 Armstrong, A. R.; Armstrong, G.; Canales, J.; Garcia, R.; Bruce, P. G. Advanced Materials 2005, 17, 862 . 
34 Wessel, C.; Zhao, L. A.; Urban, S.; Ostermann, R.; Djerdj, I.; Smarsly, B. M.; Chen, L. Q.; Hu, Y. S.; Sallard, S. Chemistry-a European Journal 2011, 17, 775.

35 Liu, S. H.; Wang, Z. Y.; Yu, C.; Wu, H. B.; Wang, G.; Dong, Q.; Qiu, J. S.; Eychmuller, A.; Lou, X. W. Advanced Materials 2013, 25, 3462.

${ }^{36}$ Ren, Y.; Liu, Z.; Pourpoint, F.; Armstrong, A. R.; Grey, C. P.; Bruce, P. G. Angewandte Chemie-International Edition 2012, 51, 2164.

37 Belak, A. A.; Wang, Y. Z.; Van der Ven, A. Chemistry of Materials 2012, 24, 2894.

38 Dylla, A. G.; Henkelman, G.; Stevenson, K. J. Accounts of Chemical Research 2013, 46, 1104.

39 Pavasupree, S.; Suzuki, Y.; Yoshikawa, S.; Kawahata, R. Journal of Solid State Chemistry 2005, 178, 3110 .

${ }^{40}$ Kavan, L.; Gratzel, M.; Gilbert, S. E.; Klemenz, C.; Scheel, H. J. J. Am. Chem. Soc. 1996, 118, 6716.

${ }^{41}$ Singh, R. K.; Narayan, J. Phys. Rev. B 1990, 41, 8843.

42 Karl, H.; Stritzker, B. Phys. Rev. Lett. 1992, 69, 2939.

43 Zhang, K.; Kim, S. J.; Zhang, Y.; Heeg, T.; Schlom, D. G.; Shen, W. Z.; Pan, X. Q. J. Phys. D-Appl. Phys. 2014, 47, 105302.

${ }^{44}$ Zhang, K.; Hao, M. R.; Guo, W.; Heeg, T.; Schlom, D. G.; Shen, W. Z.; Pan, X. Q. Physica B 2012, 407, 2825.

${ }^{45}$ Cotell, C. M.; Grabowski, K. S. MRS Bull. 1992, 17, 44.

${ }^{46}$ Chrisey, D. B.; Hubler, G. K., Pulsed Laser Deposition of Thin Films. (J. Wiley, New York, 1994).

${ }^{47}$ Krost, A.; Dadgar, A.; Strassburger, G.; Clos, R. Phys. Status Solidi A-Appl. Res. 2003, 200, 26.

${ }^{48}$ Guo, W.; Katz, M. B.; Nelson, C. T.; Heeg, T.; Schlom, D. G.; Liu, B.; Che, Y.; Pan, X. Q. Appl. Phys. Lett. 2009, 94, 122107.

${ }^{49}$ Guo, W.; Allenic, A.; Chen, Y. B.; Pan, X. Q.; Tian, W.; Adamo, C.; Schlom, D. G. Appl. Phys. Lett. 2008, 92, 072101.

${ }^{50}$ Mi, S. B.; Jia, C. L.; Vaithyanathan, V.; Houben, L.; Schubert, J.; Schlom, D. G.; Urban, K. 
Appl. Phys. Lett. 2008, 93, 101913.

51 Perriere, J.; Millon, E.; Seiler, W.; Boulmer-Leborgne, C.; Craciun, V.; Albert, O.; Loulergue, J. C.; Etchepare, J. J. Appl. Phys. 2002, 91, 690.

${ }^{52}$ Kumar, S. R. S.; Barasheed, A. Z.; Alshareef, H. N. ACS Appl. Mater. Interfaces 2013, 5, 7268.

${ }^{53}$ Ng, D. K. T.; Bhola, B. S.; Bakker, R. M.; Ho, S. T. Adv. Funct. Mater. 2011, 21, 2587.

54 Katz, M. B. Ph.D. Thesis, Materials Science and Engineering, University of Michigan, 2013.

55 Van der Pauw, L. J. Philips Res. Repts. 1958, 13, 1.

56 Hall effect measurements (http://www.nist.gov/pml/div683/hall.cfm), National Institute of Standards and Technology, 2008.

57 E. H. Putley, The Hall Effect and Related Phenomena (Butterworth \& Co, 1960).

${ }^{58}$ Lindstrom, H.; Sodergren, S.; Solbrand, A.; Rensmo, H.; Hjelm, J.; Hagfeldt, A.; Lindquist, S. E. J. Phys. Chem. B 1997, 101, 7717.

${ }^{59}$ Guo, W. Ph.D. Thesis, Materials Science and Engineering, University of Michigan, 2010.

${ }^{60}$ Srikant, V.; Speck, J. S.; Clarke, D. R. J. Appl. Phys. 1997, 82, 4286.

${ }^{61}$ Yang, D. J.; Liu, H. W.; Zheng, Z. F.; Yuan, Y.; Zhao, J. C.; Waclawik, E. R.; Ke, X. B.; Zhu, H. Y. J. Am. Chem. Soc. 2009, 131, 17885.

${ }^{62}$ Li, B. H.; Katz, M. B.; Duan, Y. W.; Du, X. F.; Zhang, K.; Chen, L.; Van der Ven, A.; Graham, G. W.; Pan, X. Q. Chemistry of Materials 2014, in revision, Manuscript ID: cm-2014-022239.

${ }^{63}$ Tanaka, H.; Taniguchi, M.; Uenishi, M.; Kajita, N.; Tan, I.; Nishihata, Y.; Mizuki, J.; Narita, K.; Kimura, M.; Kaneko, K. Angew. Chem.-Int. Edit. 2006, 45, 5998.

${ }^{64}$ Katz, M. B.; Zhang, S. Y.; Duan, Y. W.; Wang, H. J.; Fang, M. H.; Zhang, K.; Li, B. H.; Graham, G. W.; Pan, X. Q. J. Catal. 2012, 293, 145.

${ }^{65}$ Luttrell, T.; Halpegamage, S.; Sutter, E.; Batzill, M. Thin Solid Films 2014, 564, 146.

${ }^{66}$ Lotnyk, A.; Senz, S.; Hesse, D. Thin Solid Films 2007, 515, 3439.

${ }^{67}$ Erdman, N.; Poeppelmeier, K. R.; Asta, M.; Warschkow, O.; Ellis, D. E.; Marks, L. D. Nature 2002, 419, 55.

${ }^{68}$ Shinohara, R.; Yamaki, T.; Yamamoto, S.; Itoh, H.; Asai, K. J. Mater. Sci. Lett. 2002, 21, 967. 
69 Yamamoto, S.; Sumita, T.; Yamaki, T.; Miyashita, A.; Naramoto, H. J. Cryst. Growth 2002, $237,569$.

70 Tournoux, M.; Marchand, R.; Brohan, L. Prog. Solid State Chem. 1986, 17, 33.

${ }^{71}$ Kim, S. J.; Zhang, K.; Katz, M. B.; Li, B. H.; Graham, G. W.; Pan, X. Q. in prep 2014.

72 Szczygiel, I.; Winiarska, K.; Bienko, A.; Suracka, K.; Gaworska-Koniarek, D. J. Alloy. Compd. 2014, 604, 1 .

73 Jokisaari, J. R.; Bayerl, D.; Zhang, K.; Kioupakis, E.; Pan, X. Q. in prep 2014.

74 Brown, J. R. Ph.D. Thesis, Chemistry, University of Michigan, 2002.

75 Plichta, E.; Salomon, M.; Slane, S.; Uchiyama, M.; Chua, D.; Ebner, W. B.; Lin, H. W. Journal of Power Sources 1987, 21, 25.

76 Megahed, S.; Ebner, W. J. Power Sources 1995, 54, 155.

${ }^{77}$ Hao, X. G.; Lin, X. K.; Lu, W.; Bartlett, B. M. ACS Appl. Mater. Interfaces 2014, 6, 10849.

78 Scrosati, B. Nature 1995, 373, 557.

79 Sasaki, T.; Ukyo, Y; Novak, P. Nat. Mater. 2013, 12, 569.

${ }^{80}$ Whittingham, M. S. J. Electrochem. Soc. 1976, 123, 315.

${ }^{81}$ Chromik, R.; Beck, F. Electrochim. Acta 2000, 45, 2175.

${ }^{82}$ Dey, A. N. J. Electrochem. Soc. 1971, 118, 1547.

${ }^{83}$ Ferg, E.; Gummow, R. J.; Dekock, A.; Thackeray, M. M. J. Electrochem. Soc. 1994, 141, L147.

${ }^{84}$ Kanno, R.; Takeda, Y.; Ichikawa, T.; Nakanishi, K.; Yamamoto, O. J. Power Sources 1989, 26, 535.

${ }^{85}$ Bruce, P. G.; Saidi, M. Y. J. Solid State Chem. 1990, 88, 411.

${ }^{86}$ Bruce, P. G.; Saidi, M. Y. Electrochim. Acta 1991, 36, 569.

${ }^{87}$ Mizushima, K.; Jones, P. C.; Wiseman, P. J.; Goodenough, J. B. Solid State Ion. 1981, 3-4, 171.

${ }^{88}$ Murphy, D. W.; Christian, P. A.; Disalvo, F. J.; Carides, J. N. J. Electrochem. Soc. 1979, 126, 497. 
Bruce, P. G.; Krok, F.; Nowinski, J.; Gibson, V. C.; Tavakkoli, K. J. Mater. Chem. 1991, 1, 705.

${ }^{90}$ Mizushima, K.; Jones, P. C.; Wiseman, P. J.; Goodenough, J. B. Mater. Res. Bull. 1980, 15, 783.

${ }^{91}$ Needham, S. A.; Wang, G. X.; Liu, H. K.; Drozd, V. A.; Liu, R. S. J. Power Sources 2007, 174, 828.

92 Dyer, L. D.; Borie, B. S.; Smith, G. P. J. Am. Chem. Soc. 1954, 76, 1499.

${ }^{93}$ Orman, H. J.; Wiseman, P. J. Acta Crystallogr. Sect. C-Cryst. Struct. Commun. 1984, 40, 12.

94 Delmas, C.; Saadoune, I. Solid State Ion. 1992, 53, 370.

95 Delmas, C.; Saadoune, I.; Rougier, A. J. Power Sources 1993, 44, 595.

96 Aurbach, D.; Levi, M. D.; Levi, E.; Teller, H.; Markovsky, B.; Salitra, G.; Heider, U.; Heider, L. J. Electrochem. Soc. 1998, 145, 3024.

97 Levi, M. D.; Aurbach, D. Electrochim. Acta 1999, 45, 167.

98 Hatoh, K.; Niikura, J.; Yasumoto, E.; Gamo, T. J. Electrochem. Soc. 1994, 141, 1725.

99 Holzapfel, M.; Schreiner, R.; Ott, A. Electrochim. Acta 2001, 46, 1063.

100 Bianchi, V.; Bach, S.; Belhomme, C.; Farcy, J.; Pereira-Ramos, J. P.; Caurant, D.; Baffier, N.; Willmann, P. Electrochim. Acta 2001, 46, 999.

101 Carlier, D.; Menetrier, M.; Delmas, C. J. Mater. Chem. 2001, 11, 594.

102 Myung, S. T.; Komaba, S.; Kumagai, N. J. Electrochem. Soc. 2002, 149, A1349.

103 Konishi, H.; Suzuki, K.; Taminato, S.; Kim, K.; Zheng, Y. M.; Kim, S.; Lim, J.; Hirayama, M.; Son, J. Y.; Cui, Y. T.; Kanno, R. J. Power Sources 2014, 269, 293.

104 Babu, G.; Kalaiselvi, N.; Bhuvaneswari, D. J. Electron. Mater. 2014, 43, 1062.

105 Kiziltas-Yavuz, N.; Bhaskar, A.; Dixon, D.; Yavuz, M.; Nikolowski, K.; Lu, L.; Eichel, R. A.; Ehrenberg, H. J. Power Sources 2014, 267, 533.

106 Appandairajan, N. K.; Viswanathan, B.; Gopalakrishnan, J. J. Solid State Chem. 1981, 40, 117.

107 Naghash, A. R.; Lee, J. Y. Electrochim. Acta 2001, 46, 941. 
Xu, T.; Wang, W.; Gordin, M. L.; Wang, D. H.; Choi, D. W. Jom 2010, 62, 24.

109 Tukamoto, H.; West, A. R. J. Electrochem. Soc. 1997, 144, 3164.

110 Czyzyk, M. T.; Potze, R.; Sawatzky, G. A. Phys. Rev. B 1992, 46, 3729.

111 Molenda, J.; Wilk, P.; Marzec, J. Solid State Ion. 1999, 119, 19.

112 Tao, S. W.; Wu, Q. Y.; Zhan, Z. L.; Meng, G. Y. Solid State Ion. 1999, 124, 53.

113 Shibuya, M.; Nishina, T.; Matsue, T.; Uchida, I. J. Electrochem. Soc. 1996, 143, 3157.

114 Etacheri, V.; Yourey, J. E.; Bartlett, B. M. ACS Nano 2014, 8, 1491.

115 Johnson, D. C.; Prieto, A. L. J. Power Sources 2011, 196, 7736.

116 Sagane, F.; Shimokawa, R.; Sano, H.; Sakaebe, H.; Iriyama, Y. J. Power Sources 2013, 225 , 245.

117 Brutti, S.; Gentili, V.; Menard, H.; Scrosati, B.; Bruce, P. G. Advanced Energy Materials 2012, 2, 322.

118 Collaborator correspondence between Zhang, K. and Chang, D., Van der Ven, A. at University of California - Santa Barbara.

119 Conway, B. E. J. Electrochem. Soc. 1991, 138, 1539.

120 Su, X.; Wu, Q.; Zhan, X.; Wu, J.; Wei, S.; Guo, Z. Journal of Materials Science 2011, 47, 2519.

121 Armstrong, A. R.; Arrouvel, C.; Gentili, V.; Parker, S. C.; Islam, M. S.; Bruce, P. G. Chemistry of Materials 2010, 22, 6426.

${ }^{122}$ Wagemaker, M.; Kearley, G. J.; van Well, A. A.; Mutka, H.; Mulder, F. M. J. Am. Chem. Soc. 2003, 125, 840.

123 Peng, Z. Q.; Freunberger, S. A.; Chen, Y. H.; Bruce, P. G. Science 2012, 337, 563.

124 Li, S.; Wei, X. G.; Chang, Z. R.; Chen, X. N.; Yuan, X. Z.; Wang, H. J. Mater. Lett. 2014, 135, 75.

125 Padhi, A. K.; Nanjundaswamy, K. S.; Goodenough, J. B. J. Electrochem. Soc. 1997, 144, 1188.

${ }^{126}$ Bazzi, K.; Mandal, B. P.; Nazri, M.; Naik, V. M.; Garg, V. K.; Oliveira, A. C.; Vaishnava, P. P.; Nazri, G. A.; Naik, R. J. Power Sources 2014, 265, 67. 
127 Peled, E.; Menachem, C.; BarTow, D.; Melman, A. J. Electrochem. Soc. 1996, 143, L4.

128 Li, H.; Huang, X. J.; Chen, L. Q.; Wu, Z. G.; Liang, Y. Electrochem. Solid State Lett. 1999, 2, 547.

${ }^{129} \mathrm{Hu}$, Y. S.; Demir-Cakan, R.; Titirici, M. M.; Muller, J. O.; Schlogl, R.; Antonietti, M.; Maier, J. Angew. Chem.-Int. Edit. 2008, 47, 1645.

${ }^{130}$ Kim, H.; Cho, J. Nano Lett. 2008, 8, 3688.

131 Thackeray, M. M. J. Electrochem. Soc. 1995, 142, 2558.

132 Chianelli, R. R. J. Cryst. Growth 1976, 34, 239.

133 Panduwinata, D.; Gale, J. D. J. Mater. Chem. 2009, 19, 3931.

134 Arrouvel, C.; Parker, S. C.; Islam, M. S. Chemistry of Materials 2009, 21, 4778.

135 Okumura, T.; Fukutsuka, T.; Yanagihara, A.; Orikasa, Y.; Arai, H.; Ogumi, Z.; Uchimoto, Y. J. Mater. Chem. 2011, 21, 15369.

136 Personal correspondence between Zhang, K. and Li, B. H, 2014.

137 Chang, Y. C.; Peng, C. T.; Hung, I. M. J. Mater. Sci. 2014, 49, 6907.

138 Morgan, D.; Van der Ven, A.; Ceder, G. Electrochem. Solid State Lett. 2004, 7, A30.

139 Liu, H. B.; Miao, C.; Meng, Y.; He, Y. B.; Xu, Q.; Zhang, X. H.; Tang, Z. Y. Electrochim. Acta 2014, 130, 322.

${ }^{140}$ Hu, Y. Y.; Liu, Z. G.; Nam, K. W.; Borkiewicz, O. J.; Cheng, J.; Hua, X.; Dunstan, M. T.; Yu, X. Q.; Wiaderek, K. M.; Du, L. S.; Chapman, K. W.; Chupas, P. J.; Yang, X. Q.; Grey, C. P. Nat. Mater. 2013, 12, 1130.

141 Yamada, A.; Chung, S. C.; Hinokuma, K. J. Electrochem. Soc. 2001, 148, A224.

${ }^{142}$ Huang, H.; Yin, S. C.; Nazar, L. F. Electrochem. Solid State Lett. 2001, 4, A170.

${ }^{143}$ Chung, S. Y.; Bloking, J. T.; Chiang, Y. M. Nat. Mater. 2002, 1, 123.

144 Wang, R. Y.; Wessells, C. D.; Huggins, R. A.; Cui, Y. Nano Lett. 2013, 13, 5748.

145 Suzuki, Y.; Shinoda, Y. Sci. Technol. Adv. Mater. 2011, 12.

146 Hazen, R. M.; Yang, H. X. Science 1997, 277, 1965.

147 Reddy, M. A.; Kishore, M. S.; Pralong, V.; Caignaert, V.; Varadaraju, U. V.; Raveau, B. Chem. 
Mat. 2008, 20, 2192.

148 Snyder, G. J.; Toberer, E. S. Nat. Mater. 2008, 7, 105.

149 Nishihata, Y.; Mizuki, J.; Akao, T.; Tanaka, H.; Uenishi, M.; Kimura, M.; Okamoto, T.; Hamada, N. Nature 2002, 418, 164.

${ }^{150}$ Katz, M. B.; Graham, G. W.; Duan, Y. W.; Liu, H.; Adamo, C.; Schlom, D. G.; Pan, X. Q. J. Am. Chem. Soc. 2011, 133, 18090.

151 Tanaka, H.; Taniguchi, M.; Uenishi, M.; Kajita, N.; Tan, I.; Nishihata, Y.; Mizuki, J.; Narita, K.; Kimura, M.; Kaneko, K. Angew. Chem.-Int. Edit. 2006, 45, 5998.

${ }^{152}$ Katz, M. B.; Zhang, S. Y.; Duan, Y. W.; Wang, H. J.; Fang, M. H.; Zhang, K.; Li, B. H.; Graham, G. W.; Pan, X. Q. J. Catal. 2012, 293, 145.

153 Shimura, K.; Yoshida, H. Energy Environ. Sci. 2010, 3, 615.

154 Hsu, K. F.; Loo, S.; Guo, F.; Chen, W.; Dyck, J. S.; Uher, C.; Hogan, T.; Polychroniadis, E. K.; Kanatzidis, M. G. Science 2004, 303, 818.

155 Dresselhaus, M. S.; Chen, G.; Tang, M. Y.; Yang, R. G.; Lee, H.; Wang, D. Z.; Ren, Z. F.; Fleurial, J. P.; Gogna, P. Advanced Materials 2007, 19, 1043.

${ }^{156}$ Cahill, D. G. Rev. Sci. Instrum. 1990, 61, 802.

157 Mi, S. B.; Jia, C. L.; Vaithyanathan, V.; Houben, L.; Schubert, J.; Schlom, D. G.; Urban, K. Appl. Phys. Lett. 2008, 93.

158 Tomio, T.; Miki, H.; Tabata, H.; Kawai, T.; Kawai, S. J. Appl. Phys. 1994, 76, 5886.

159 Oh, D. W.; Ravichandran, J.; Liang, C. W.; Siemons, W.; Jalan, B.; Brooks, C. M.; Huijben, M.; Schlom, D. G.; Stemmer, S.; Martin, L. W.; Majumdar, A.; Ramesh, R.; Cahill, D. G. Appl. Phys. Lett. 2011, 98, 221904.

160 Zhang, K.; Shen, W. Z. Appl. Phys. Lett. 2008, 92, 083101.

161 Zhang, K.; Hao, M. R.; Guo, W.; Heeg, T.; Schlom, D. G.; Shen, W. Z.; Pan, X. Q. Physica B 2012, 407, 2825.

${ }^{162}$ Liu, Y. F.; Sahoo, P.; Makongo, J. P. A.; Zhou, X. Y.; Kim, S. J.; Chi, H.; Uher, C.; Pan, X. Q.; Poudeu, P. F. P. J. Am. Chem. Soc. 2013, 135, 7486.

163 Ohta, S.; Nomura, T.; Ohta, H.; Koumoto, K. J. Appl. Phys. 2005, 97, 034106. 